\title{
Optimal Emergency Shutdown Valve Configuration for Pressurised Pipelines
}

A thesis submitted to University College London for the degree of

Doctor of Philosophy

By

Jianhao Yu

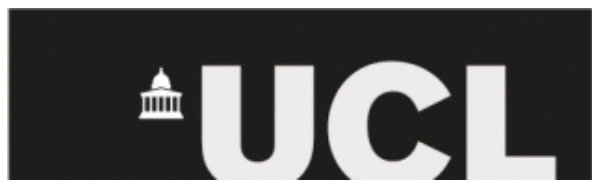

Department of Chemical Engineering

University College London

Torrington Place

London WC1E 7JE

September 2021 
I, Jianhao Yu confirm that the work presented in this thesis is my own. Where information has been derived from other sources, I confirm that this has been indicated in the thesis. 
Abstract

Pressurised pipelines are the primary mode of choice for transporting large quantities of hazardous fluids across the globe. The failure of such pipelines can lead to the release of significant amounts of flammable or toxic inventories, which may in turn present significant risks to life, environment and property. In order to mitigate such risks, various types of emergency shutdown valves (ESDVs), including Check Valves (CVs), Automatic Shut-off Valves (ASVs) and Remote Control Valves (RCVs), are installed along such pipelines as the front-line emergency mitigation tool.

Accounting for the critically important ensuing in-pipe transient fluid flow, this thesis presents the development and application of a multi-objective optimisation study for selecting the ESDV type, number and spacing as well as its combinations and operational settings for striking a balance between the minimum valve capital cost against the efficacy in minimising and ultimately isolating outflow following pipeline failures.

Two types of pipeline failures, including Full Bore Rupture (FBR) and puncture are considered. Ethylene and natural gas (typical flammable and explosive hydrocarbons) as well as Carbon Dioxide $\left(\mathrm{CO}_{2}\right)$ are chosen as the transported fluids. $\mathrm{CO}_{2}$ is selected given its hazardous nature (an asphyxiant at $>7 \% \mathrm{v} / \mathrm{v}$ ) and the extensive use of pressurised pipelines being proposed as the main method for transporting large quantities of captured $\mathrm{CO}_{2}$ for permanent geological storage as part of the Carbon Capture and Storage chain.

The pipeline decompression model employed is based on the Homogeneous Equilibrium Mixture assumption, where the constituent fluid phases are assumed to be at both thermodynamic and mechanical equilibrium. The Peng-Robinson Equation of State along with relevant hydrodynamic and thermodynamic relations are employed to determine the required fluid thermophysical properties and phase equilibrium data. The impact of the valve closure on the in-pipe fluid flow dynamics is accounted for through the implementation of appropriate boundary conditions. The resulting system of conservation equations is solved numerically using the Method of Characteristics.

The first part of the study focuses on the investigation of ESDV dynamic response and characteristics on the fluid behaviour following the accidental failure of a hypothetical nevertheless realistic ethylene pipeline. This is divided into two parts: in the first, the results 
based on the simulation of the pressure surges upon CV closure are presented for a wide practical range of ethylene pipeline operating pressures and temperatures. It is found that for high operating pressures (over 90 bar), pressure surges upon CV closure can lead to a large thrust or bending forces acting on the pipeline segments, potentially damaging the pipeline in the event of exceeding the maximum safe design operating pressure. In the second part, the impact of ASV and RCV activation pressure is assessed by determining the amount of escaped inventory prior to complete isolation in the event of pipeline FBR and different generic sizes of puncture failures. The results show that for FBR and relatively large puncture failures $(>50$ $\%$ pipe i.d.), decreasing valve activation pressure results in an insignificant reduction in the total inventory loss prior to valve closure. In the case of small puncture diameter failures (less than $5 \%$ pipe i.d.), ASV may not be self-activated given the relatively small pressure drop across the valve throughout the decompression process.

The above is followed by the analysis of the efficacy of ESDVs using different inline valve combinations and spacings by performing two sets of investigations. The first includes the simulations of fluid flow behaviour upon valve closure using a hypothetical $\mathrm{CO}_{2}$ pipeline puncture decompression scenario. Different valve spacings and combinations of inline RCVs and CVs are investigated, and the results are presented and discussed in terms of the release pressure and temperature, the discharge mass flowrate and, more importantly, the total mass released, as a function of time during decompression. Next, based on the FBR failure of a highpressure ethylene pipeline, the efficacy of CVs, RCVs and ASVs and their combinations for the emergency isolation is investigated by comparing the amount of escaped inventory prior to complete valve closure.

Building on the above work, the study culminates in optimising inline ESDV configuration by developing a multi-objective optimisation method combined with Principal Component Analysis and applying it to a real, $1016 \mathrm{~mm}$ i.d., $150.2 \mathrm{~km}$ long natural gas transmission pipeline in China operating at 80 bar and 307.24 K. Starting with defining a set of 6 characteristic variables for ESDV settings, PCA is first employed in order to reduce the number of variables to 3, whilst retaining good agreement for the problem solution and emphasis on computational simplification. Next, a description of the optimisation problem is performed, detailing the objective functions used, including valve capital cost, and the various parameter realistic values assumed. The results of the multi-objective optimisation are presented using scatter plots providing a geometrical visualisation of the Pareto Front and Set. Such results 
serve as a highly informative tool in assisting pipeline operators in selecting the optimal inline ESDV configurations for pressurised pipelines. 


\section{Impact Statement}

This thesis presents the development and application of a rigorous and computationally efficient multi-objective optimisation model combined with Principal Component Analysis to determine the optimal Emergency Shutdown Valve (ESDV) configuration for pressurised transportation pipelines.

As high-pressure pipelines are the primary mode of choice for transporting significant quantities of hazardous fluids across the globe, their safe operation is thus of paramount importance. ESDVs are often employed along such pipelines as the front-line emergency mitigation tool for the rapid isolation of outflow following accidental pipeline failures. As such, the methodology developed in this thesis can be directly applied for the quantitative failure consequence assessment of high-pressure transportation pipelines to assist pipeline operators to select the appropriate ESDV configuration. The model takes into account a myriad of design and operational characteristics parameters including the ESDV activation and closure times, combination, number, spacing and failure rate to strike a balance between minimising cost and minimising the amount of inventory escaping prior to complete pipeline isolation. A knowledge of the subsequent predicted time variant amount of inventory escaping following emergency isolation for the optimised ESDV configuration is particularly important as it serves as the source term for determining the consequences of the pipeline failure. These ultimately dictate the minimum pipeline safe distances to populated areas and emergency response planning.

In addition, the methodology developed in this work provides a fundamental modelling technique for complex multi-objective problems and the results can serve as a highly informative tool in practice. This work successfully demonstrates the capability of employing the variable reduction techniques in the multi-objective optimisation study, with emphasis on computational simplification whilst still ensuring an improved outcome for a real-world multiobjective problem. 


\section{Acknowledgements}

I wish to thank the following people who have contributed so much in many ways to facilitate the completion of this thesis.

To my supervisor, Prof. Haroun Mahgerefteh for giving me the opportunity to study in this field and your excellent supervision.

To my parents and brother: Thank you for your never-ending support and motivation.

To my dear friends, Jiahuan Yi, Wentian Zheng, Kaiqiao $\mathrm{Wu}$ and Haiyue $\mathrm{Yu}$, and to my colleagues, Nor, Revelation, Yao, Fidal and Dr. Richard Porter. Thank you for friendship and encouragement.

To Dr. Sergey Martynov, for all the advice and criticism through the course of my time at UCL.

To the technical and admin. staff of the Department of Chemical Engineering, UCL.

Finally, to my dear girlfriend, Haewon Shin. Thank you for your understanding and support throughout my studies. 


\section{Table of Contents}

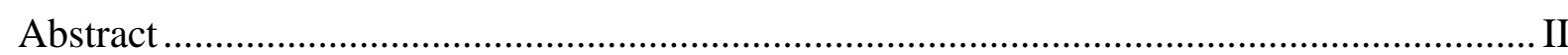

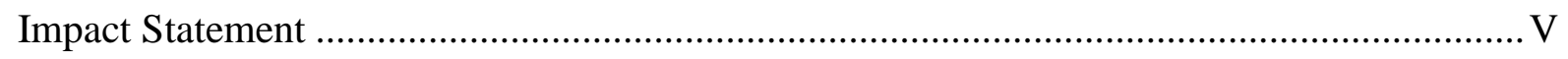

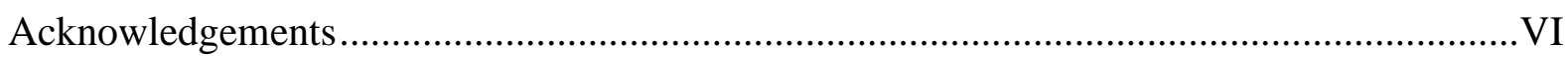

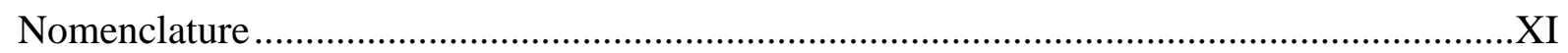

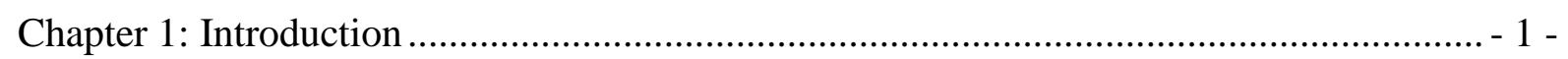

Chapter 2: Fundamental Theory for Pipeline Transient Flow Modelling and its Numerical

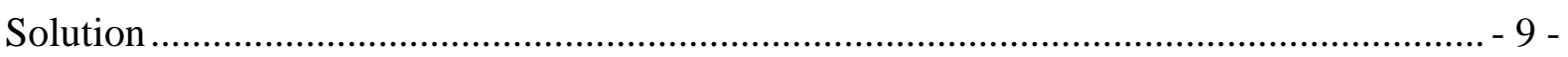

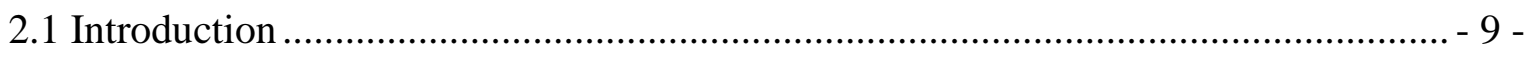

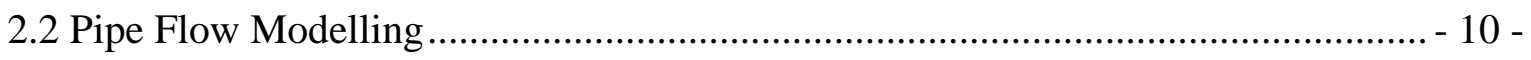

2.1.1 General Conservation Equations for Fluid Dynamics .................................. - 10 -

2.1.2 Pipe Flow Modelling ............................................................................. 11 -

2.3 Homogeneous Equilibrium Mixture Model ............................................................ - 15 -

2.3.1 Modelling Assumptions........................................................................... - 15 -

2.3.2 Conservation Equations........................................................................ 16 -

2.3.3 Hydrodynamic and Thermodynamic Relations for Homogeneous Mixtures... - 17 -

2.3.4 The Peng - Robinson Equation of State.................................................. - 21 -

2.3.5 The Steady State Isothermal Flow Model .................................................. - 22 -

2.4 Numerical Solution of Hyperbolic Partial Differential Equations ........................... - 24 -

2.4.1 Methods of Discretisation..................................................................... -25 -

2.4.2 Numerical Formulation and Implementation of the Method of Characteristics- 27 - 
2.4.3 Finite Difference Solution of Compatibility Equations $-30-$

2.5 Boundary Conditions for Simulating Pipeline Failure $-31-$

2.5.1 Pipeline Upstream Conditions $-32-$

2.5.2 Pipeline Downstream Conditions (Failure Plane) $-34-$

2.5.3 Full Bore Rupture $-35-$

2.5.4 Pipeline Puncture $-38-$

2.5.5 Emergency Shutdown Valve Closure Modelling $42-$

2.6 Applications of the HEM Model $49-$

2.6.1 Mahgerefteh et al. (1999) $50-$

2.6.2 Terenzi (2005) $57-$

2.6.3 Lund at el. (2011) $59-$

2.6.4 Teng et al. (2016) $62-$

2.6.5 Xu et al. (2014) $64-$

2.6.6 Summary of the HEM model applications $-68-$

2.7 Concluding Remarks $71-$

Chapter 3: Multi-objective Optimisation and Its Applications $73-$

3.1 Introduction $-73-$

3.2 General Approaches and Visualisation Techniques $-75-$

3.2.1 General Approaches for Selecting the Optimal Solutions. $-75-$

3.2.2 Visualisation of Pareto Front $-76-$

3.3 Applications of Multi-objective Optimisation Methods - 79 - 


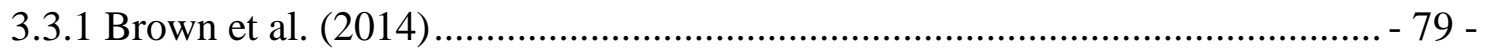

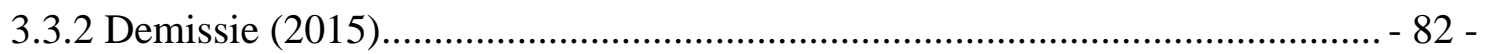

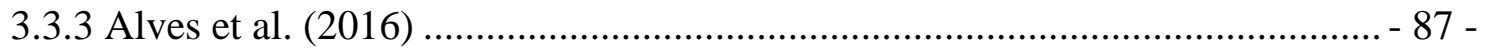

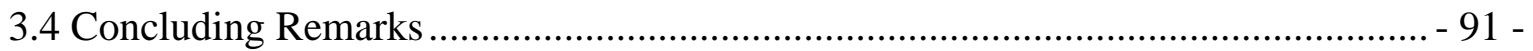

Chapter 4: Modelling and Study of Emergency Shutdown Valve Dynamic Response Following Accidental Failure of High-Pressure Pipelines ................................................................... - 93 -

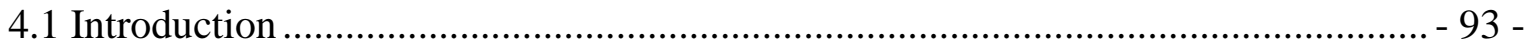

4.2 The Study of Pressure Surges upon CV Closure ................................................... - 94 -

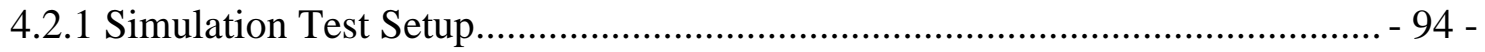

4.2.2 Results and Discussion .................................................................................... 96 -

4.3 The Study of ASV and RCV Activation Pressure .............................................. - 102 -

4.3.1 Simulation Test Setup.......................................................................... - 102 -

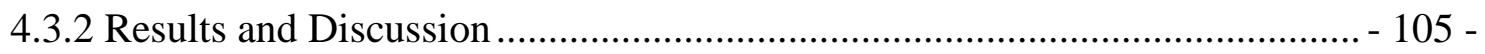

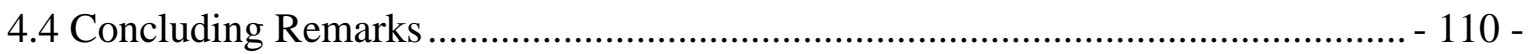

Chapter 5: The Efficacy of Emergency Shutdown Valve following the Accidental Failure of

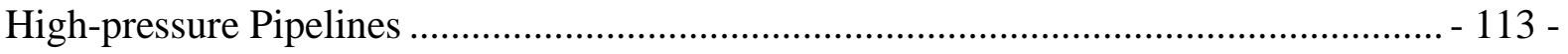

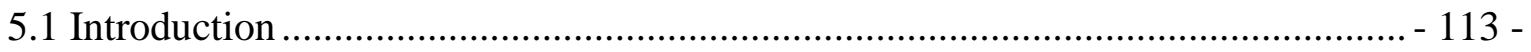

5.2 The Study of CV and RCV Spacing …............................................................... 114 -

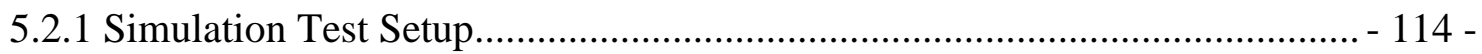

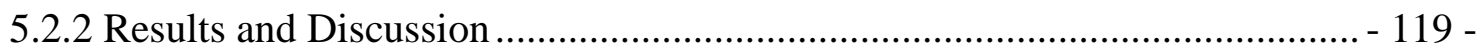

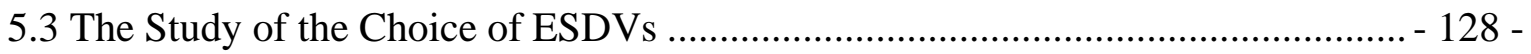

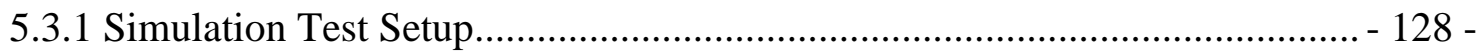


5.3.2 Results and Discussion $-130-$

5.4 Concluding Remarks $-133-$

Chapter 6: Optimising Emergency Shutdown Valve Configurations for Pressurised Pipelines Using Multi-objective Optimisation and Principal Component Analysis -136 -

6.1 Introduction $-136-$

6.2 Theory $-138-$

6.2.1 Multi-objective Optimisation $-138-$

6.2.2 Principal Component Analysis (PCA) $-139-$

6.3 Results and Discussion $-143-$

6.3.1 Case Study $-143-$

6.3.2 Principal Component Analysis (PCA) Results $-150-$

6.3.3 Multi-objective Optimisation Results $-154-$

6.4 Concluding Remarks $-158-$

Chapter 7: Conclusions and Future Work. $-161-$

7.1 Conclusions $161-$

7.2 Suggestions for Future Work $168-$

7.2.1 Leak Detection Modelling $168-$

7.2.2 Reliability Analysis for Other Control Devices $-168-$

7.2.3 Combining Pipeline Maintenance Optimisation Models with ESDV MultiObjective Optimisation Study $-169-$

References - 170 - 
$A=$ pipe cross-sectional area $\left(\mathrm{m}^{2}\right)$

$A_{f}=$ area of flow through the valve $\left(\mathrm{m}^{2}\right)$

$A_{o l}=$ area of the orifice $\left(\mathrm{m}^{2}\right)$

$a=$ speed of sound $\left(\mathrm{m} \mathrm{s}^{-1}\right)$

$C_{d}=$ discharge coefficient

$C_{P}=$ specific heat at constant pressure $\left(\mathrm{J} \mathrm{kg}^{-1} \mathrm{~K}^{-1}\right)$

$C_{v}=$ specific heat at constant volume $\left(\mathrm{J} \mathrm{kg}^{-1} \mathrm{~K}^{-1}\right)$

$D=$ valve spacing distance $(\mathrm{m})$

$D L F=$ dynamic magnification factor

$E=$ specific total energy $\left(\mathrm{J} \mathrm{kg}^{-1}\right)$

$e=$ specific internal energy $\left(\mathrm{J} \mathrm{kg}^{-1}\right)$

$F=$ body force $(\mathrm{N})$

$f_{w}=$ fanning friction factor

$g=$ gravitational acceleration $\left(\mathrm{m} \mathrm{s}^{-2}\right)$

$h=$ specific enthalpy $\left(\mathrm{J} \mathrm{kg}^{-1}\right)$

$h_{o l}=$ enthalpy at the release plane $\left(\mathrm{J} \mathrm{kg}^{-1}\right)$

$I=$ identity matrix

$J=$ vector of objective function

$K=$ binary interaction parameter 
$k=$ isothermal coefficient of volumetric expansion $\left(\mathrm{m} \mathrm{s}^{2} \mathrm{~kg}^{-1}\right)$

$M_{g}=$ molecular weight of the gas phase $\left(\mathrm{kg} \mathrm{mol}^{-1}\right)$

$M_{l}=$ molecular weight of the liquid phase $\left(\mathrm{kg} \mathrm{mol}^{-1}\right)$

$M_{o}=$ initial mass of inventory prior to failure $(\mathrm{kg})$

$M_{\text {total }}=$ total inventory loss from a ruptured pipeline $(\mathrm{kg})$

$\bar{M}_{\text {total }}=$ average of the total inventory loss for all failure locations tested (kg)

$\dot{m}=$ discharge flow rate $\left(\mathrm{kg} \mathrm{s}^{-1}\right)$

$n=$ average lifetime of the equipment (year)

$P=$ pressure $($ bar $)$

$P_{a m b}=$ ambient pressure (bar)

$P_{C}=$ critical pressure $($ bar $)$

$P_{o l}=$ discharge pressure at the release plane (bar)

$P_{\text {res }}=$ reservoir pressure (bar)

$\operatorname{Pr}=$ proportion of the total variation

$\Delta p_{a}=$ valve activation pressure (bar)

$Q=$ volumetric flow rate $\left(\mathrm{m}^{3} \mathrm{~s}^{-1}\right)$

$Q_{h}=$ fluid/wall heat transfer $(\mathrm{J})$

$q=$ heat flux $\left(\mathrm{W} \mathrm{m}^{-2}\right)$

$R=$ universal gas constant

Re $=$ Reynolds number

$r=$ discount rate 
$r_{i n}=$ pipe inner radius $(\mathrm{m})$

$S=$ source terms

$S=$ specific entropy $\left(\mathrm{J} \cdot \mathrm{K}^{-1}\right)$

$T=$ temperature $(\mathrm{K})$

$T_{a m b}=$ ambient temperature $(\mathrm{K})$

$T_{C}=$ critical temperature $(\mathrm{K})$

$T_{f}=$ fluid temperature $(\mathrm{K})$

$T_{o l}=$ temperature at the release plane $(\mathrm{K})$

$t=$ time $(\mathrm{s})$

$t_{a}=$ valve activation time (s)

$t_{c}=$ valve complete closure time (s)

$U_{h}=$ overall heat transfer coefficient

$u=$ velocity $\left(\mathrm{m} \mathrm{s}^{-1}\right)$

$u_{o l}=$ velocity at the release plane $\left(\mathrm{m} \mathrm{s}^{-1}\right)$

$u_{v}=$ valve linear closure rate $\left(\mathrm{cm} \mathrm{s}^{-1}\right)$

$V=$ specific volume of the fluid $\left(\mathrm{m}^{3}\right)$

$V_{P N}=$ single valve cost $(£)$

$x=$ mole fraction

$y=$ Principal Component

$Z_{g}=$ compressibility of the gas phase

$Z_{l}=$ compressibility of the liquid phase 


\section{Greek Symbols}

$\alpha=$ volume fraction

$\beta_{x}=$ fluid/wall friction $\left(\mathrm{kg} \mathrm{m}^{-2} \mathrm{~s}^{-2}\right)$

$\Gamma=$ orthogonal data matrix

$\gamma=$ ratio of specific heats

$\varepsilon=$ pipe roughness height $(\mathrm{m})$

$\theta=$ angle of inclination of the pipeline to the horizontal plane $\left(^{\circ}\right)$

$\lambda_{j}=$ variance of the $j$ th Principal Component

$\rho=$ density $\left(\mathrm{kg} \mathrm{m}^{-3}\right)$

$\rho_{g}=$ gas density $\left(\mathrm{kg} \mathrm{m}^{-3}\right)$

$\rho_{l}=$ liquid density $\left(\mathrm{kg} \mathrm{m}^{-3}\right)$

$\rho_{o}=$ fluid density at the prevailing pipeline transportation pressure and temperature $\left(\mathrm{kg} \mathrm{m}^{-3}\right)$

$\Sigma=$ covariance matrix

$\tau=$ stress tensor $\left(\mathrm{N} \mathrm{m}^{-2}\right)$

$\varphi=$ isochoric thermodynamic function

$\chi=$ liquid mass fraction

$\omega=$ acentric factor 


\section{Chapter 1: Introduction}

According to International Energy Agency (IEA) (2020), the global energy demand reached 13,946.2 Mtoe (Million tonnes of oil equivalent) in 2019. Within the energy mix, about $80 \%$ is in the form of fossil fuels including oil, natural gas and other hydrocarbons. As the fossil fuels production sites are usually not collocated with the power plants, long distance transportation is required. Oil and gas may be transported via various options including ships, railroads, trucks and high-pressure pipeline networks. Among these options, high-pressure pipeline transportation is considered to be the safest and most economically viable (Transportation Research Board, 2004). As such, sophisticated networks of high-pressure pipelines are widely used in oil and gas industry across the globe. For example, in the UK, the National Transmission System (NTS) has a total length of over $7600 \mathrm{~km}$ high-pressure natural gas pipelines operating at pressures up to 94 bar (National Grid, 2021). Other important industrial chemicals such as ethane, propane and ethylene are also broadly transported via highpressure (usually over 100 bar) transmission pipelines (Crawley et al., 2003).

Besides hydrocarbons, in recent years, high-pressure pipelines are being used as the major transportation mode for considerable amount of Carbon Dioxide $\left(\mathrm{CO}_{2}\right)$ (approximately 68 million tonnes per year) as part of the Carbon Capture and Storage (CCS) chain across the globe (Global CCS Institude, 2018). The extensive use of fossil fuels has led to numerous amounts of $\mathrm{CO}_{2}$ emissions. According to IEA (2020), the global $\mathrm{CO}_{2}$ emissions in 2019 were around 33 gigatonnes, increasing by approximately $15 \%$ since 2010 . The excessive $\mathrm{CO}_{2}$ emissions can lead to global warming and climate change, posing significant threats to living species and the environment. $\mathrm{CCS}$ is considered as one of the most promising $\mathrm{CO}_{2}$ mitigation methods for combating the issue of climate change. In brief, CCS involves the transportation of the captured $\mathrm{CO}_{2}$ (e.g. from coal fire power plants or other energy intensive industrial emitters) via highpressure pipelines for the subsequent storage in geological formations (e.g. depleted gas reservoir). According to the Carbon Capture \& Storage Association (2020), CCS can help the detention of $\mathrm{CO}_{2}$ emissions for up to $90 \%$ from the use of fossil fuels in electricity generation and industrial processes. Given CCS' significant potential in mitigating global warming, the 
worldwide $\mathrm{CO}_{2}$ pipeline network length is estimated to exceed $100,000 \mathrm{~km}$ carrying the captured $\mathrm{CO}_{2}$ by 2030 (International Energy Agency, 2009).

Both fossil fuel and $\mathrm{CO}_{2}$ high-pressure pipelines may by necessity pass through densely populated urban areas in order to maintain their economic viability. Given that hydrocarbons are highly flammable and explosive, and $\mathrm{CO}_{2}$ is an asphyxiant at high concentrations (> 7\% v/v; Harper, 2011), a major safety concern is the risks associated with the failure of these pipelines. The release of a large amount of flammable or toxic inventories in the event of such failures present significant risks to life, environment and property. For example, on 9 September 2010, a 30 inch diameter natural gas transmission pipeline owned by Pacific Gas \& Electric Company ruptured and released vast quantities of inventory in San Bruno, California, the U.S. (Pipeline and Hazardous Materials Safety Administration, 2017). A violent explosion occurred at the time of the rupture, and the escaping gas ignited which quickly engulfed nearby houses. 8 fatalities, 51 injuries and over $\$ 220$ million property damage from the incident were reported. The cause of the pipeline rupture was identified as the failure of numerous defective welds when the operating pressure in the pipeline was increased. On 19 August 2000, a natural gas transmission pipeline rupture failure led to a huge explosion in Carlsbad, New Mexico, the U.S., causing severe damage to the neighbourhood including nearby steel suspension bridges for gas pipelines crossing the river (National Transportation Safety Board, 2003). The explosion itself and the subsequent fire also resulted in 12 fatalities.

Only in the U.S., 5743 significant pipeline incidents have been reported in the past 20 years. These incidents in total have resulted in 258 fatalities, 1143 injuries and more than $\$ 10$ billion damage to property (Pipeline and Hazardous Materials Safety Administration, 2021). Given this, the risks associated with pipeline failure must be assessed to ensure the safe design and operation of high-pressure transmission pipelines; a main focus being the determination of the minimum safe distances in the event of an accidental failure for pipeline routing.

Central to the above risk assessment is the accurate prediction of the decompression characteristics (e.g. pressure and temperature variations as a function of time) and outflow from the failed pipeline. Consequently, the development of mathematical models for this purpose has been the focus of considerable attention (see for example Mahgerefteh et al., 1999; Terenzi, 2005; Mahgerefteh et al., 2008; Munkejord et al., 2010b; Lund et al., 2011; Brown, et al., 2014). 
The mathematical problem addressed in almost all these models involve solving the mass, momentum and energy conservation equations using appropriate numerical schemes, such as the Method of Characteristics (MOC). Pipeline outflow models based on the Homogeneous Equilibrium Mixture (HEM) assumption are the most popular, robust and computationally efficient. The HEM assumption states that the constituent fluid phases are fully dispersed, remaining in thermodynamic and mechanical equilibrium with each other during the pipeline decompression process.

Given the above, in recent years, several pipeline outflow models adopting the HEM assumption have been developed with different levels of sophistication such as accounting for fluid phase transition, fluid/wall heat transfer and friction. Mahgerefteh and his co-workers (Mahgerefteh et al., 1999, 2011; Oke et al., 2003) at University College London (UCL) have applied the HEM assumption in the development of their models for hydrocarbon and $\mathrm{CO}_{2}$ pipeline decompression. Real-fluid Equation of States (EoSs) (e.g. Peng-Robison Equation of State (PR EoS); (Peng and Robinson, 1976)) were employed for the prediction of the required fluid thermal properties and phase equilibrium data. These HEM based models have been extensively validated against multiple experimental data obtained from large-scale highpressure pipeline decompression experiments for both hydrocarbons and $\mathrm{CO}_{2}$ producing good agreement with the model predictions. In subsequent publications, Mahgerefteh et al. (1997, 2000, 2006, 2010, 2011, 2012) extended their basic pipeline flow model to account for emergency shut down, a simplified analytically based vessel blowdown model, decompression of pipeline networks and simulating ductile fracture propagation in the pipe wall.

More recently, $\mathrm{Xu}$ et al. (2014) presented the development and validation of a pipeline depressurisation model based on the HEM assumption. In the case of single-phase flow, the PR-EoS was implemented to calculate fluid thermodynamic properties and phase equilibrium data. For two-phase flow, the method developed by Mehra et al. (1983) was used to calculate liquid-vapor phase equilibrium. The model was validated against the Botros's experiment pressure and temperature data (Botros et al., 2007). The results showed reasonably good agreement between the model predictions and experiment data.

The above studies have demonstrated the efficacy of the HEM based models in successfully simulating the highly transient fluid flow behaviour following pipeline failures. In terms of 
mitigating their failure consequence, inline emergency isolation devices such as Emergency Shutdown Valves (ESDVs) are extensively employed as the front-line tool.

Three main types of ESDVs are in common use (Oland et al., 2012). These include:

- Automatic Shut-off Valves (ASVs) which close upon the detection of a change in the line pressure. The ASV activation time corresponds to the duration of the fluid decompression to a pre-set pressure at the valve location.

- Remote Control Valves (RCVs). These valves are equipped with an actuator which close the valve upon the receipt of a signal from the operator. The RCVs activation time is equal the sum of the time lapsed for detection of the set pressure drop at the valve location and the operator response time to activate valve closure either automatically or manually.

- Check Valves (CVs). CVs are designed to prevent back-flow from the downstream section of a pipeline following its rupture in high-consequence areas, such as water basins and population centres (Oland et al., 2012). CVs are activated upon the detection of flow reversal at the valve location. In comparison to ASVs and RCVs, CVs offer the fasted 'cut-off' of a back-flow downstream of the ruptured section of the pipeline (Mahgerefteh et al., 1997). However, in practice, the near-instantaneous closure of a $\mathrm{CV}$ upon rupture may produce a pressure surge which, could in turn create a sufficiently large thrust force acting on segments of the pipeline, resulting in its deformation and vibrations (Koetzier et al., 1986; Mahgerefteh et al., 1997; Ord, 2006). In extreme cases, such pressure surges may exceed the maximum safe operating pressure of the pipeline leading to secondary catastrophic failure.

The appropriate type and the strategic positioning of inline ESDVs, along with their number, striking a balance between risk reduction against the valve capital cost, requires the precise knowledge of the in-pipe fluid flow dynamics during valve closure.

As part of the development of the HEM model by Mahgerefteh et al.(1997), the ensuing inpipe fluid dynamics upon CVs and ball-type RCVs closure were accounted for through the implementation of appropriate boundary conditions including the time-dependent change in the available flow area during the valve closure. In the case of a ball-type RCV, the model was 
successfully validated against available experiment data for emergency isolation of a test $\mathrm{CO}_{2}$ pipeline (Mahgerefteh et al., 2016). However, fundamentally important questions of crucial practical significance have yet to be addressed. These include:

- the resulting pressure surges upon CV closure

- the impact of ASV and RCV activation pressure in isolating the outflow flow following pipeline failure

- the efficacy of using different combinations of inline ESDV along with their strategic positioning in isolating the outflow flow following pipeline failure

Given that the flow dynamic response is sensitive to valve types (Thorley, 1989; Chern et al., 2007), and the significant capital cost of ESDVs which can become prohibitive depending on their type (CVs are much cheaper than ASVs or RCVs; Smith and Zappe, 2004) and number of units employed, the 'optimum inline ESDV configuration' must be carefully determined to remain cost effective while still providing sufficient protection in the event of a pipeline failure. Such challenge can be defined as a multi-objective problem.

Recently, Brown et al. (2014) presented a multi-objective optimisation study balancing valve spacing in $\mathrm{CO}_{2}$ pipelines as a trade-off between risk reduction versus ESDV installation and maintenance costs. The results indicated that beyond a certain threshold, decreasing valve spacing provided only a marginal reduction in risk whilst substantially increasing costs. However, with the exception of valve spacing, other essential optimisation variables related to ESDV configurations, including the ESDV type \& combination, number and operational settings must also be considered for the multi-objective problem. Significantly, increasing the number of optimisation variables leads to numerous additional mathematical complexities, whilst making solutions difficult to visualise and analyse. To overcome this limitation, variable reduction techniques, such as Principal Component Analysis (PCA), can be adopted to identify redundant variables which can be disregarded whilst ensuring an improved outcome.

To address the above challenges, the aim of this study is to develop and apply a multi-objective optimisation method for selecting the ESDV type, number and spacing as well as its combinations and operational settings for striking a balance among three objective functions namely: 
i. the ESDV capital cost

ii. the ESDVs failure rate

iii. the amount of total inventory escaping prior to complete valve closure following pipeline failure

Based on modelling of the in-pipe transient fluid flow behaviour using the HEM model, the main project objectives are to:

- $\quad$ simulate and investigate ESDV dynamic response following pipeline failures

- $\quad$ simulate and analyse the efficacy of ESDVs based on their type, number, spacing and combination in minimising and ultimately isolating outflow following pipeline failures

- develop and apply using a real high-pressure transmission pipeline as a case study, a multi-objective optimisation method combined with PCA for determining the optimal ESDV configurations for high-pressure pipelines

Two types of pipeline failures; Full Bore Rupture (FBR) and puncture are considered in this work. FBR is chosen as it is the most catastrophic type of pipeline failure possible in the offshore industry (Ramírez-Camacho et al., 2017). Although in comparison to pipeline FBR failure, pipeline puncture is much less catastrophic, its significantly higher failure frequency (Goodfellow et al., 2019) requires equal attention when performing quantitative failure consequence assessment for high-pressure transmission pipelines.

Ethylene, natural gas and Carbon Dioxide $\left(\mathrm{CO}_{2}\right)$ are chosen as the fluids being transported. $\mathrm{CO}_{2}$ is selected given its physiological hazard; it is an asphyxiant at concentrations $>7 \% \mathrm{v} / \mathrm{v}$ (Harper, 2011) and the planned extensive use of pressurised pipelines for its transport for permanent geological storage as part of the Carbon Capture and Storage chain. Ethylene and natural gas on the other hand represent flammable and explosive fluids.

This thesis is divided into 7 chapters:

In Chapter 2, the theoretical basis for the HEM model and its numerical solution employed in this work is presented. The above commences with a review of the background theory for pipeline decompression modelling, including the fundamental conservation equations to 
describe the fluid dynamics for pipe flow. This is followed by the presentation of the HEM model's building blocks covering the pertaining assumptions, the mass, momentum and energy conservation equations, the hydrodynamic and thermodynamic relations for homogeneous mixtures and the PR EoS for predicting the required fluid thermophysical properties and phase equilibrium data. Next, the formulation and implementation of the MOC to solve the conservation equations alongside the appropriate boundary conditions required to simulate the outflow from a pipeline and ESDV closure dynamic response following failure are presented.

The various boundary conditions include pipeline upstream conditions (closed end \& reservoir), pipeline downstream conditions (closed end \& open end), FBR \& puncture and ESDVs (CVs, ASVs and RCVs) closure dynamic response. The same chapter includes a review of relevant studies focusing on the applications of the HEM model in predicting high-pressure pipeline decompression behaviour. In particular, the model performance is discussed with a focus on the degree of agreement of its predictions against the available experimental data for pipeline decompression tests \& field measurements.

Chapter 3 presents a review of the development of multi-objective optimisation methods. This includes the general approaches for selecting the optimal solutions, the specific techniques to visualise the possible solutions and a survey of the relevant studies available in the open literature focusing on the applications of multi-objective optimisation methods for pipeline network design and operation.

In Chapter 4, the investigation of ESDV dynamic response and operational characteristics such as ASV and RCV activation pressure on the fluid behaviour following the accidental failure of a hypothetical nevertheless realistic ethylene pipeline is presented. This is divided into two parts: in the first, the results based on the simulation of the pressure surges upon CV closure are presented for practical range of ethylene pipeline operating pressures and temperatures. In the second part, the impact of the magnitudes of ASV and RCV activation pressure on the total amount of inventory escaping prior to complete isolation following pipeline failure is investigated and the salient features are discussed. Both FBR and various generic puncture diameters are considered.

Chapter 5 presents the analysis of the efficacy of ESDVs using different inline valve combinations and spacing by performing two sets of investigations. The first includes the simulation of fluid flow behaviour upon valve closure using a hypothetical $\mathrm{CO}_{2}$ pipeline 
puncture failure scenario. Different valve spacing of inline RCVs and CVs are investigated, and the results are presented and discussed in terms of the variation of fluid release pressure and temperature, the discharge mass flowrate and cumulative mass discharged during the decompression process. Next, based on the FBR failure of a high-pressure ethylene pipeline, the efficacies of ASVs, RCVs and CVs and their combinations for the emergency isolation are investigated by comparing the amount of total inventory escaping prior to complete valve closure.

Chapter 6 presents the development of the multi-objective optimisation method combined with PCA in order to produce the optimal ESDV configurations for high-pressure pipelines. This is based on balancing the ESDV capital cost against the efficacy in minimising and ultimately isolating outflow and the total ESDV failure rate for different combinations of inline ESDVs. The constructed model is next applied to a real, $1016 \mathrm{~mm}$ i.d., $150.2 \mathrm{~km}$ long natural gas transmission pipeline in China operating at 80 bar and 307.24 K. Starting with defining a set of 6 characteristic variables for ESDV settings, PCA is first employed in order to reduce the number of these variables whilst retaining good agreement for the problem solution, whilst ensuring computational simplification. Next, a description of the optimisation problem is presented, detailing the objective functions being used and the various parameter realistic values assumed. Finally, the results of the multi-objective optimisation for the ESDV equipped pipeline are presented and discussed using scatter plots providing a geometrical visualisation of the possible optimal solutions.

Chapter 7 presents a summary of the important conclusions and suggestions for future work. 
Chapter 2:

\section{Fundamental Theory for Pipeline Transient Flow Modelling and its Numerical Solution}

\subsection{Introduction}

As discussed in Chapter 1, Emergency Shutdown Valves (ESDVs) are employed for the rapid isolation of outflow following the accidental failure of pressurised pipelines. Central to choosing the appropriate type and strategic positioning of such valves along the pipeline is the accurate modelling of the highly transient flow behaviour during the decompression process. Amongst the available flow models in the literature, the Homogeneous Equilibrium Mixture (HEM) model which assumes thermodynamic and mechanical equilibrium between the constituent fluid phases is the most popular method applied for this purpose.

The development of the HEM model involves two main parts.

The first part requires:

i. the formulation of the basic conservation equations governing the flow

ii. the constituent relations accounting for fluid/pipe wall heat transfer and friction

iii. a suitable Equations of State (EoS) for predicting real fluid thermophysical and phase equilibrium data

iv. the implementation of the closure boundary conditions

The second part entails the selection and implementation of an efficient and accurate method that resolves or simplifies the above set of equations into easily solvable forms.

Accordingly, this chapter is organised as follows.

In Sections 2.2, the basic theory for the key building blocks for pipeline decompression modelling are first presented, including the general conservation equations for describing fluid flow. This is followed by a discussion of the main features of the existing pipeline multi-phase 
flow models. These include the HEM model; the Homogeneous Relaxation Mixture (HRM) model; the Drift Flux Mixture (DFM) model and the Two-Fluid Mixture (TFM) model.

Section 2.3 details the mathematical development of the HEM model, including model assumptions; conservation equations; hydrodynamic and thermodynamic relations for homogeneous mixtures; the general features and the pertaining assumptions of the PengRobinson EoS (PR EoS) for predicting the fluid thermophysical properties and phase equilibrium data. Also included is the mathematical formulation of the isothermal steady state flow model prior to pipeline failure; an essential requirement for simulating the subsequent transient flow behaviour following pipeline failure.

Sections 2.4 comprises a review of various numerical methods for solving the conservation equations governing single/two-phase homogeneous flow in pipelines. Particular attention is paid to the detailed formulation and implementation of the Method of Characteristics (MOC) as a solution methodology given its accuracy and robustness. In Section 2.5, the boundary conditions required to simulate the outflow from a pipeline and ESDV closure dynamic response following failure are presented.

Section 2.6 provides a review of the relevant studies focusing on the applications of the HEM model in predicting high-pressure pipeline decompression behaviour. In each study, a main focus is the evaluation of the model performance based on the degree of agreement with experimental data for pipeline decompression tests \& field results. Conclusions are drawn in Section 2.7 .

\subsection{Pipe Flow Modelling}

\subsubsection{General Conservation Equations for Fluid Dynamics}

For any type of flow, the general mass, momentum and energy conservation equations for fluid dynamics of the $k^{\text {th }}$ fluid phase within an arbitrary flow control volume with a control surface are given by (Ishii et al., 2006): 


$$
\frac{\partial \alpha_{k} \overline{\overline{\rho_{k}}}}{\partial t}+\nabla \cdot\left(\alpha_{k} \overline{\overline{\rho_{k}}} \underline{\underline{\boldsymbol{u}_{k}}}\right)+I_{k}=0
$$

$$
\begin{gathered}
\frac{\partial \alpha_{k} \overline{\overline{\rho_{k}}} \underline{\boldsymbol{u}_{k}}}{\partial t}+\nabla \cdot\left(\alpha_{k} \overline{\overline{\rho_{k}}} \underline{\boldsymbol{u}_{k}} \boldsymbol{u}_{k}\right)+\nabla \cdot\left(\alpha_{k} \overline{\overline{P_{k}}} \boldsymbol{I}\right)-\nabla \cdot \alpha_{k}\left(\boldsymbol{\tau}_{k}^{t}+\overline{\overline{\boldsymbol{\tau}_{k}}}\right)+I_{k}^{\boldsymbol{u}_{k}}-\alpha_{k} \overline{\overline{\boldsymbol{F}_{k}}} \\
=0
\end{gathered}
$$

$$
\begin{aligned}
& \frac{\partial \alpha_{k} \overline{\overline{\rho_{k}}} \underline{E_{k}}}{\partial t}+\nabla \cdot\left(\alpha_{k} \overline{\overline{\rho_{k}}} \underline{\boldsymbol{u}_{k} E_{k}}\right)+\nabla \cdot\left(\alpha_{k} \overline{\overline{P_{k}}} \boldsymbol{I} \cdot \underline{\boldsymbol{u}_{k}}\right)-\nabla \cdot\left(\alpha_{k} \overline{\overline{\boldsymbol{\tau}_{k}}} \cdot \underline{\boldsymbol{u}_{k}}\right)+\nabla \\
& \cdot \alpha_{k}\left(\boldsymbol{q}_{k}^{t b}+\overline{\overline{\boldsymbol{q}_{k}}}\right)+\overline{\bar{P}} \frac{\partial \alpha_{k}}{\partial t}+I_{k}^{E_{k^{*}}}-\alpha_{k} \overline{\overline{\boldsymbol{F}_{k}}} \cdot \underline{\boldsymbol{u}_{k}}=0
\end{aligned}
$$

where, $\alpha_{k}, \rho_{k}$, and $P_{k}$ are respectively the volume fraction, density, and pressure of the $k^{\text {th }}$ fluid phase. $I_{k}, I_{k}^{\boldsymbol{u}_{k}}$ and $I_{k}^{E_{k^{*}}}$ are respectively donated as the interface mass, momentum and energy exchange (interaction) terms. The velocity, heat flux and body force are respectively symbolised by the vectors, $\boldsymbol{u}_{k}, \boldsymbol{q}_{k}$ and $\boldsymbol{F}_{k} . \boldsymbol{\tau}_{k}$ and $\boldsymbol{I}$ respectively represent the stress tensor and the identity matrix. The subscript $k$ is the $k^{\text {th }}$ fluid phase while the superscript $t b$ is the turbulence. ' =, and ' _, are the averaging operators, which respectively represent phaseweighted time-averaging and mass-weighted time-averaging. The total energy, $E_{k}$ is defined as:

$$
E_{k}=e_{k}+\left|\boldsymbol{u}_{k}\right|^{2} / 2
$$

where, $e_{k}$ is the internal energy and the term $\left|\boldsymbol{u}_{k}\right|^{2} / 2$ is the kinetic energy.

\subsubsection{Pipe Flow Modelling}

\subsubsection{General Conservation Equations for Pipe Flow}

As the conservation equations presented above are a set of coupled, high-order and non-linear Partial Differential Equations (PDEs), they cannot be solved analytically (Ferziger et al., 2020). As such, these conservations equations are often solved by neglecting the inconsequential terms 
based on appropriate assumptions to reduce the computational complexity for most practical engineering problems.

In terms of pipe flow, equations 2.1 to 2.3 are often assumed to be one-dimensional and momentum diffusion, energy diffusion and turbulence terms are commonly neglected to enable simpler mathematical derivation (Mahgerefteh et al., 1999; Cleaver et al., 2003; Oke et al.,

2003). Under such assumptions, the terms $I_{k}^{\boldsymbol{u}_{k}}$ and $I_{k}^{E_{k^{*}}}$ in equations 2.2 and 2.3 respectively serve as fluid/wall friction and heat transfer (Ishii and Hibiki, 2011). Besides, the volumetric terms and the interface exchange terms in equations 2.1 to 2.3 can be combined as the source terms (Saha, 1997). Given the above, the mass, momentum and energy conservation equations describing one-dimensional pipe flow of the $k^{\text {th }}$ fluid phase can be respectively simplified as (Zheng, 2018):

$\frac{\partial \alpha_{k} \rho_{k}}{\partial t}+\frac{\partial \alpha_{k} \rho_{k} u_{k}}{\partial x}=S_{M, k}$

$\frac{\partial \alpha_{k} \rho_{k} u_{k}}{\partial t}+\frac{\partial\left(\alpha_{k} \rho_{k} u_{k}^{2}+\alpha_{k} P_{k}\right)}{\partial x}=S_{F, k}$

$\frac{\partial \alpha_{k} \rho_{k} E_{k}}{\partial t}+\frac{\partial\left(\alpha_{k} \rho_{k} u_{k} E_{k}+\alpha_{k} u_{k} P_{k}\right)}{\partial x}+P_{k} \frac{\partial \alpha_{k}}{\partial t}=S_{E, k}$

where, $S_{M, k}, S_{F, k}$ and $S_{E, k}$ are respectively defined as the source terms for the mass, momentum and energy conservation equations.

\subsubsection{Multi-phase Flow Modelling}

Multi-phase flow often occurs during pipeline decompression due to liquid evaporation or vapour condensation. Modelling such phenomenon requires more than one set of conservation equations as a result of the complex interface interactions. For instance, the flows can be considered as homogeneous (thermodynamic and mechanical) equilibrium if the rates of interphase mass, momentum and energy exchange are sufficient high. Heterogenous flows (e.g. slug flow, stratified flow and annular flow) will occur if the rate of interphase momentum 
exchange is not high enough to retain mechanical equilibrium. When the rates of interface mass and energy exchange are delayed, thermodynamic non-equilibrium will occur resulting in fluid metastable states and distinctive phasic temperatures.

As such, various approaches have been developed in recent studies focusing on modelling the above phenomena incorporating different interface interactions. These studies can be generally categorised as follows (Munkejord et al., 2016; Pham and Rusli, 2016): Homogeneous Equilibrium Mixture (HEM) model; Homogeneous Relaxation Mixture (HRM) model; Drift Flux Mixture (DFM) model and Two-Fluid Mixture (TFM) model. The main features of these four models are presented in table 2.1. 
Table 2.1: Main features of common flow models for predicting pipeline decompression and their application examples.

\begin{tabular}{|c|c|c|}
\hline Multi-phase Flow Model & Main Features & Model Application Examples \\
\hline HEM model & $\begin{array}{l}\text { - The constituent fluid phases are assumed to be at thermal (i.e. same } \\
\text { temperature and pressure) and mechanical (i.e. no phase slip) } \\
\text { equilibrium } \\
\text { - The most frequently applied model as a result of its simplicity and low } \\
\text { computational demand }\end{array}$ & $\begin{array}{l}\text { Mahgerefteh et al. (1997, 1999, } \\
\text { 2011) } \\
\text { Martynov et al. (2014) } \\
\text { Lund at el. (2011) } \\
\text { Terenzi (2005) }\end{array}$ \\
\hline HRM model & $\begin{array}{l}\text { - Employed to model thermodynamic non-equilibrium due to delayed } \\
\text { phase transition during pipeline decompression }\end{array}$ & $\begin{array}{l}\text { Angielczyk et al. (2010) } \\
\text { Brown et al. (2013) }\end{array}$ \\
\hline TFM model & $\begin{array}{l}\text { - Both thermodynamic and mechanical non-equilibrium between the } \\
\text { constituent fluid phases. Interface interactions are modelled explicitly } \\
\text { - More physically relevant and has a wider range of applicability in } \\
\text { pipeline decompression modelling }\end{array}$ & $\begin{array}{l}\text { Brown et al. (2014) } \\
\text { Munkejord and Hammer (2015) } \\
\text { Zheng (2018) }\end{array}$ \\
\hline
\end{tabular}


Given its simplicity and relatively low computational demand (Mahgerefteh et al., 1999; Zheng, 2018), the HEM model is the most frequently applied model for predicting pipe flow, which has evolved in recent years, with different levels of sophistication such as accounting for fluid phase transition, fluid/wall heat transfer and friction. Furthermore, the HEM model has been shown to produce reasonably good agreement against available field and experimental data for simulating the decompression failure of high-pressure pipelines (see (Mahgerefteh et al., 1999, 2012; Oke et al., 2003; Xu et al., 2014; Teng et al., 2016), for example). Given the above, the HEM model is adopted in this study.

Accordingly, given its relevance, in the following sections, the detailed mathematical development for the HEM model and its numerical solution is presented. The mathematical developments for the HRM, DFM and TFM models can be found elsewhere (Bilicki and Joseph, 1990; Downar-Zapolski et al., 1996; Paillère et al., 2003; Munkejord et al., 2010a; Brown et al., 2013; Yeom and Chang, 2013; Zheng, 2018).

\subsection{Homogeneous Equilibrium Mixture Model}

\subsubsection{Modelling Assumptions}

The development of the in-pipe transient flow following pipeline failure is based on the following assumptions:

$>$ Steady state isothermal flow prior to pipeline failure

$>$ The flow is assumed to be one-dimensional

$>$ For multi-phase flow, the constituent fluid phases are assumed to be at thermal (i.e. same temperature and pressure) and mechanical (i.e. no phase slip) equilibrium

$>$ The pipeline is firmly clamped and of uniform cross-sectional area 


\subsubsection{Conservation Equations}

Assuming one-dimensional flow and neglecting the viscous terms, the mass, momentum and energy conservation equations of the HEM model are respectively given by (Versteeg et al., 1995):

$\frac{\partial \rho}{\partial t}+\frac{\partial \rho u}{\partial x}=0$

$\frac{\partial \rho u}{\partial t}+\frac{\partial\left(\rho u^{2}+P\right)}{\partial x}=\beta_{x}-\rho g \sin \theta$

$\frac{\partial\left[\rho\left(e+\frac{u^{2}}{2}\right)\right]}{\partial t}+\frac{\partial\left[\rho u\left(e+\frac{u^{2}}{2}\right)+P u\right]}{\partial x}=Q_{h}-u \beta_{x}$

where, $\rho, e, u, P, \theta, g, Q_{h}$ and $\beta_{x}$ are the bulk average density, internal energy, velocity, pressure, angle of inclination of the pipeline to the horizontal plane, gravitational acceleration, fluid/wall heat transfer and friction, respectively. $t$ and $x$ are respectively the time and space.

The total derivative of density with respect to time, $\partial \rho / \partial t$ in equation 2.8 , can be expressed in terms of either fluid pressure and entropy or fluid pressure and enthalpy. As demonstrated by Oke (2004), the pressure and enthalpy formulation produces the best accuracy when compared to real data alongside considerable reduction in computational run-time. As such, the same formulation is implemented in this work. Thus, the conservation equations of mass, momentum and energy with pressure, enthalpy and velocity being the primitive variables can be respectively expressed as (Oke, 2004):

$$
\begin{aligned}
& (\rho T+\varphi)\left(\frac{\partial P}{\partial t}+u \frac{\partial P}{\partial x}\right)-\rho \varphi\left(\frac{\partial h}{\partial t}+u \frac{\partial h}{\partial x}\right)+\rho^{2} a^{2} T \frac{\partial u}{\partial x}=0 \\
& \frac{\partial \rho u}{\partial t}+\frac{\partial\left(\rho u^{2}+P\right)}{\partial x}=\beta_{x}-\rho g \sin \theta \\
& \rho \frac{d h}{d t}-\frac{\partial P}{\partial t}-u \frac{\partial P}{\partial x}=Q_{h}-u \beta_{x}
\end{aligned}
$$


where, $h$ is the enthalpy. $a$ and $\varphi$ are respectively the speed of sound and isochoric thermodynamic function (see later).

\subsubsection{Hydrodynamic and Thermodynamic Relations for Homogeneous Mixtures}

\subsubsection{The Speed of Sound}

The speed of propagation of waves transmitting information through the fluid is largely dependent on the speed of sound.

For single-phase homogeneous mixtures, the speed of sound, $a$ is derived analytically as given by (Picard and Bishnoi, 1987):

$$
a^{2}=\frac{\gamma}{k \rho}
$$

where, the ratio of specific heats, $\gamma$ and isothermal coefficient of volumetric expansion $k$, are respectively given by (Walas, 1985):

$$
\begin{aligned}
& \gamma=\frac{C_{P}}{C_{V}} \\
& k=-\rho\left(\frac{\partial V}{\partial P}\right)_{T}
\end{aligned}
$$

Here, $V$ is the specific volume of the fluid. $C_{P}$ and $C_{v}$ are respectively the specific heats at constant pressure and volume. The term $\left(\frac{\partial V}{\partial P}\right)_{T}$ can be obtained analytically from differentiation of the EoS (see Section 2.3.4).

For two-phase mixtures, the speed of sound is evaluated numerically at a given temperature and pressure given by (Mahgerefteh et al., 2000): 


$$
a^{2}=\left(\frac{\Delta P}{\rho(T, P)-\rho\left(T^{*}, P-\Delta P\right)}\right)_{s}
$$

where, $\Delta P$ is the infinitesimal change in pressure and $T^{*}$ represents the corresponding temperature obtained from the pressure-entropy flash calculation.

\subsubsection{The Isochoric Thermodynamic Function, $\varphi$}

The isochoric thermodynamic function $\varphi$ (equation 2.11), for single-phase homogeneous fluids, is defined as (Picard and Bishnoi, 1987):

$$
\varphi=\left(\frac{\partial P}{\partial s}\right)_{\rho}=\frac{\rho \xi T a^{2}}{C_{P}}
$$

where, $\xi$ is the isobaric coefficient of volumetric expansion which can be given by:

$$
\xi=\frac{1}{V}\left(\frac{\partial V}{\partial T}\right)_{P}
$$

For two-phase mixtures, by adopting Maxwell's relations, the isochoric thermodynamic function can be expressed as (Walas, 1985):

$$
\varphi=\rho^{2}\left(\frac{\partial T}{\partial \rho}\right)_{s}=\rho^{2}\left(\frac{\Delta T}{\Delta \rho}\right)_{s}
$$

The above equation can be solved numerically by performing an isentropic flash calculation, same as the solution of equation 2.17 . 


\subsubsection{Two-phase Mixture Density}

In line with the HEM assumption (Section 2.3.1), for vapour-liquid two-phase flow, the pressure and temperature for both phases are equal. Accordingly, the mixture density can be defined as (Atti, 2006):

$$
\rho=\frac{\rho_{g} \rho_{l}}{\rho_{g}(1-\chi)+\rho_{l} \chi}
$$

where, $\chi$ is the liquid mass fraction. $\rho_{g}$ and $\rho_{l}$ are the density of the gas and liquid density (at given $T$ and $P$ ) which can be respectively given by:

$$
\begin{gathered}
\rho_{g}=\frac{P M_{g}}{Z_{g} R T} \\
\rho_{l}=\frac{P M_{l}}{Z_{l} R T}
\end{gathered}
$$

Here, $P$ and $T$ are the equilibrium pressure and temperature, respectively. $Z_{g}$ and $Z_{l}$ are respectively the compressibility of the gas phase and liquid phase. $M_{g}$ and $M_{l}$ are respectively the molecular weights of the gas and liquid phases.

\subsubsection{Fluid/wall Friction}

Fluid/wall friction can be included as a body force in the momentum conservation equation for one-dimensional pipeline decompression flow models. It is correlated with the friction factor, $f_{w}$, determined by appropriate empirical relations that depend on the flow regime.

The fluid/wall friction, $\beta_{x}$ is defined as (Oke, 2004):

$$
\beta_{x}=-2 \frac{f_{w} \rho u|u|}{D}
$$

where, $f_{w}$ and $D$ are respectively the fanning friction factor and pipeline diameter. 
For laminar pipe flow (Reynolds number, $\mathrm{Re}<2300$ ), the fanning friction factor is independent of the pipe roughness and can be given by (Rohsenow et al., 1998):

$$
f_{w}=\frac{16}{\operatorname{Re}}
$$

For transition $(2300<\operatorname{Re}<4000)$ and turbulent flows $(\operatorname{Re}>4000)$ in rough pipes (the pipe roughness height, $\varepsilon$ is greater than thickness of viscous sublayer), the fanning friction factor is calculated from Chen (1979):

$$
\frac{1}{\sqrt{f_{w}}}=3.48-1.7372 \ln \left(\frac{\varepsilon}{r_{\text {in }}}-\frac{16.2446}{R e} \ln A\right)
$$

where,

$$
A=\frac{\left(\varepsilon / r_{i n}\right)^{1.0198}}{6.0983}+\left(\frac{7.149}{R e}\right)^{0.8981}
$$

Here, $r_{i n}$ is the pipe inner radius.

In the case of transition and turbulent flows in smooth pipes ( $\varepsilon$ is less than thickness of viscous sublayer), the fanning friction factor is evaluated using the recommendation of Rohsenow et al. (1998):

$$
\frac{1}{\sqrt{f_{w}}}=1.7372 \ln \left(\frac{R e}{1.694 R e-3.8215}\right)
$$

\subsubsection{Fluid/Wall Heat Transfer}

Newton's cooling law is widely used for determining the heat fluid/wall exchange, $Q_{h}$ for a fluid flowing in a pipe (Fairuzov, 1998; Mahgerefteh et al., 1999): 


$$
Q_{h}=\frac{4}{D_{\text {in }}} U_{h}\left(T_{a m b}-T_{f}\right)
$$

where, $U_{h}$ is the overall heat transfer coefficient. $T_{a m b}$ and $T_{f}$ are respectively the ambient temperature and temperature of the fluid.

\subsubsection{The Peng - Robinson Equation of State}

In the above, some key thermodynamic variables for pipeline outflow modelling were defined. The calculation of the pertinent fluid thermophysical properties and phase equilibrium data requires an appropriate EoS. In this study the PR EoS (Peng and Robinson, 1976) is employed given its established applicability to high pressure hydrocarbon mixtures along with its computational efficiency (Walas, 1985; Mahgerefteh et al., 1999).

The PR EoS for a pure component is given by (Peng and Robinson, 1976):

$$
\begin{aligned}
& P=\frac{R T}{V-b_{V}}-\frac{a_{V}(T)}{V^{2}+2 b_{v}-\left(b_{v}\right)^{2}} \\
& a_{V}(T)=a\left(T_{c}\right) \times \alpha\left(T_{r}, \omega\right)
\end{aligned}
$$

At the critical point, parameters $a$ and $b$ are expressed in terms of the critical properties:

$$
\begin{aligned}
& a\left(T_{c}\right)=0.45724 \frac{R^{2} T_{C}^{2}}{P_{C}^{2}} \\
& b\left(T_{c}\right)=0.07780 \frac{R T_{C}}{P_{C}}
\end{aligned}
$$

For mixtures, parameters $a$ and $b$ are defined as:

$$
a=\sum_{i}^{N} \sum_{j}^{N}\left(1-K_{i j}\right) \sqrt{\left(a_{i} \alpha_{i}\right)\left(a_{j} \alpha_{j}\right)}
$$




$$
b=\sum_{i}^{N} x_{i} b_{i}
$$

where, $P_{c}, T_{c}, V, R$ and $\omega$ are the critical pressure, critical temperature, molar volume, the universal gas constant and the acentric factor, respectively. $a_{i}$ and $b_{i}$ are the constants determined for each component. Also, $K_{i j}$ and $x_{i}$ are the binary interaction parameter and component mole fractions, respectively. The alpha function, $\alpha$, is given by Soave (1972):

$$
\alpha=\left(1+\kappa\left(\frac{T}{T_{C}}\right)^{0.5}\right)^{2}
$$

where,

$$
\kappa=0.48+1.574 \omega-0.175 \omega^{2}
$$

From equation 2.16, the term $\left(\frac{\partial V}{\partial P}\right)_{T}$ can be obtained by differentiating the PR EoS (equation 2.30) to give:

$$
\left(\frac{\partial V}{\partial P}\right)_{T}=\left[\frac{-R T}{\left(V-b_{V}\right)^{2}}-\frac{a_{V} \alpha\left(2 V+2 b_{V}\right)}{\left[V^{2}+2 b_{V} V-\left(b_{V}\right)^{2}\right]^{2}}\right]^{-1}
$$

\subsubsection{The Steady State Isothermal Flow Model}

As mentioned in Section 2.3.1, isothermal steady state flow is assumed prior to pipeline failure. The corresponding pressure drop based on the continuity and momentum equations presented in Section 2.3.2 is determined following Oke (2004) given its successful validation against real data. The following is a brief overview.

When all the fluid properties are time invariant, the steady state expression for one-dimensional mass continuity can be written as: 
$u \frac{d \rho}{d x}+\rho \frac{d u}{d x}=0$

The above equation can be rewritten by separating variables and integrating (Oke, 2004):

$$
\rho_{i} u_{i}=\rho_{i-1} u_{i-1}
$$

Here, the subscripts $i$ and $i-1$ respectively denote the current and penultimate grid point. It is noted that equation 2.40 is the governing equation for mass conservation, valid for steady state flow in a uni-diameter pipeline.

Similar to equation 2.39 , the steady state one-dimensional momentum equation can also be expressed by setting the time derivatives to zero such that:

$$
\rho u \frac{d u}{d x}+\frac{d P}{d x}=\beta_{x}-\rho g \sin \theta
$$

In order to calculate the pressure drop under isothermal steady state condition prior to pipeline failure, substituting the expression for $\beta_{x}$ (equation 2.24), equation 2.41 can be integrated and rewritten as (Oke, 2004):

$$
\begin{aligned}
& \frac{1}{2}\left[\left(\frac{\rho}{\rho^{2} K_{3}+K_{2}}\right)_{P_{i}}+\left(\frac{\rho}{\rho^{2} K_{3}+K_{2}}\right)_{P_{i-1}}\right]\left(P_{i}-P_{i-1}\right) \\
& +\frac{K_{1}}{2 K_{2}}\left[2 \ln \left(\frac{\rho_{i}}{\rho_{i-1}}\right)-\ln \left(\frac{\rho_{i}^{2} K_{3}+K_{2}}{\rho_{i-1}^{2} K_{3}+K_{2}}\right)\right]=x_{i}-x_{i-1}
\end{aligned}
$$

where, $K_{1}, K_{2}$ and $K_{3}$ are constants and can be given by:

$$
\begin{aligned}
& K_{1}=-(\rho u)^{2} \\
& K_{2}=-\frac{2 f_{w}(\rho u)^{2}}{D_{\text {in }}} \\
& K_{3}=g \sin \theta
\end{aligned}
$$


Based on the above, the following presents the calculation algorithm for determining the isothermal steady state pressure drop along the pipe prior to failure (Oke, 2004):

1. Provide the fluid/flow data at pipeline inlet (e.g. fluid pressure, temperature and velocity, etc.).

2. Divide the pipeline into a number of equidistant grids with the distance $\Delta x=x_{i}-x_{i-1}$.

3. Guess $P_{i}$ (downstream pressure at the next grid point).

4. Calculate left hand side of equation 2.42 in conjunction with the PR EoS.

5. If equation 2.42 is satisfied, the fluid velocity $u_{i}$ can be updated by equation 2.40 . Otherwise go back to step 3 and update the guessed $P_{i}$ until equation 2.40 is satisfied.

6. Update fluid properties at this grid point. Then repeat steps 3 to 5 to calculate the pressure drop at the next grid point until fluid properties at the final grid point can be calculated.

\subsection{Numerical Solution of Hyperbolic Partial Differential Equations}

In Section 2.3.2, the mass, momentum and energy conservation equations were presented to describe the transient fluid flow during pipeline decompression in the event of failure. These equations are a set of quasi-linear hyperbolic Partial Differential Equations (PDEs) which contain nonlinear teams. To solve them, a numerical method needs to be introduced (Flatt, 1986; Mahgerefteh et al., 1999). There are a variety of numerical methods that can be used to solve the PDEs. The most commonly employed methods are: the Finite Difference Method (FDM) (see for example Chen, 1993; Bendlksen et al., 2004), the Finite Volume Method (FVM) (see for example LeVeque, 2002; Toro, 2009) and the Method of Characteristics (MOC) (see for example Zucrow and Hoffman, 1976; Flatt, 1986; Chen et al., 1992).

In the case of the FDM, the spatial domain is divided into a series of nodes forming a grid. Then by substituting finite approximations, the derivatives appearing in the PDEs take values 
at the nodal points which result in a system of algebraic equations. Turning to the FVM, the system is discretised into a set of control volumes. A system of algebraic equations is generated by approximating the integral of the PDEs over each cell. In recent years, the application of the FVM to hyperbolic PDEs has received considerable attention (LeVeque, 2002; Toro, 2009; Brown, 2011). However, according to Mahgerefteh and Atti (2006), these methods are unsuitable for modelling the transient flow following pipeline failure due to the numerical diffusion.

The MOC, on the other hand, is particularly suited to the solution of hyperbolic equations with two independent variables (for example, $x$ and $t$ in this case). The MOC essentially converts the PDEs into Ordinary Differential Equations (ODEs). This is achieved through particular coordinate changes so that the co-ordinates represent curves in the $x$ - $t$ plane along which the ODEs hold. The MOC has been proved particularly suitable for systems containing complex boundary conditions (such as modelling inline ESDVS), as each boundary condition can be applied individually to each characteristic curve moving into the computational domain. (Saha, 1997).

Given the above and its unrivalled popularity, the MOC is adopted in this study for numerical solution of the conservation equations governing single/two-phase flow following pipeline failure. The following provides an overview of the MOC, including the grid discretisation method, the numerical formulation and implementation of the MOC and the finite difference method to resolve the compatibility equations.

\subsubsection{Methods of Discretisation}

There are two main grid discretisation methods for the MOC: the first one is the Characteristic Grid (CG) method (Wylie and Streeter, 1993), also be known as the Natural Method of Characteristics. The second one is the Inverse Marching method, also known as the Method of Specified Time Intervals (MST) (Flatt, 1986).

Figure 2.1 shows the schematic representation of the $\mathrm{CG}$ method. For this method, the position of the solution is not known in advance. This is determined by the intersection of the left and the right running characteristics with the origin located at points where the solution is already 
known. A free-floating grid is created in the $\mathrm{x}$-t plane as shown in figure 2.1. The advantage of this method is the particularly accuracy as the solution progresses along the characteristics naturally.

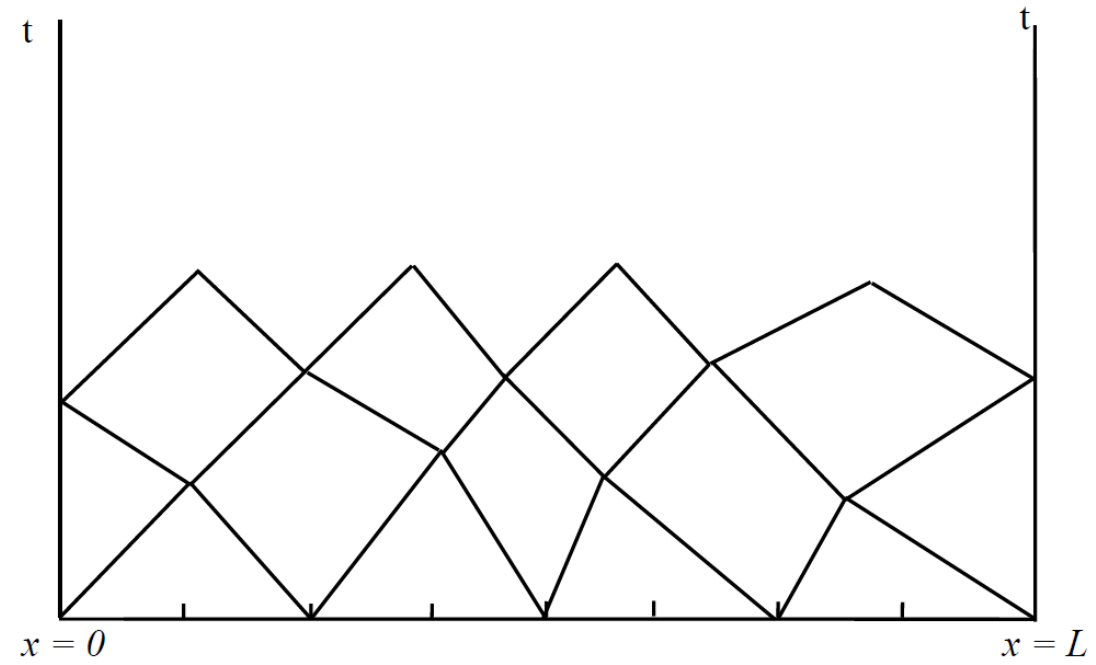

Figure 2.1: Schematic representation of the Characteristic Grid method.

Figure 2.2 shows the schematic representation of the MST. As can be observed in the figure, the locations of the solution points are specified in advance and characteristic lines are traced backwards in time to their origin through the previous points. The method necessitates interpolation to locate the intersection of all three characteristics on the previous timeline. Thus, this can result in a loss of accuracy.

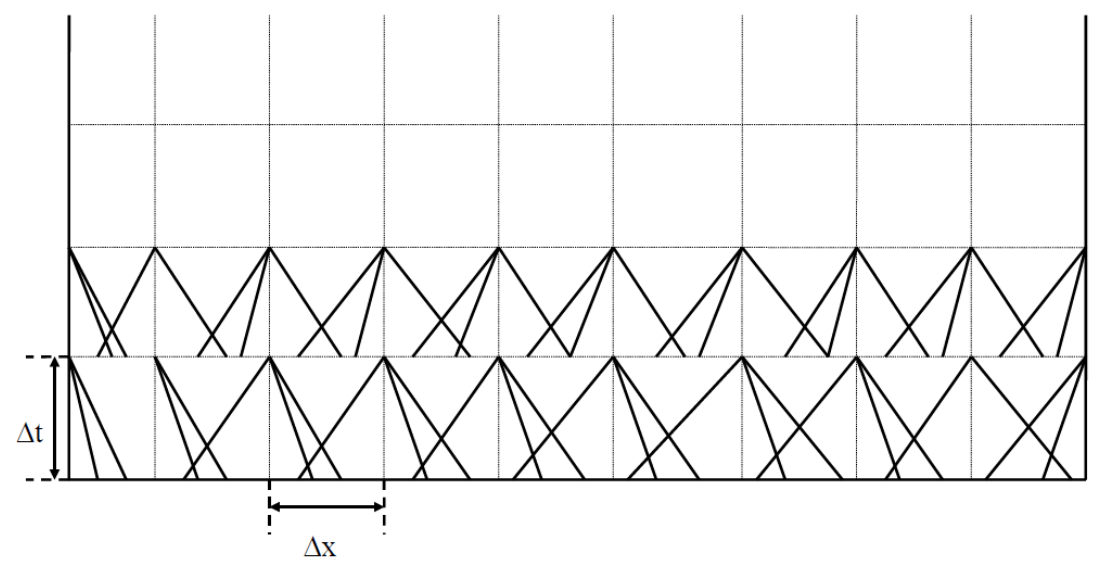

Figure 2.2: Schematic representation of the Method of Specified Time Intervals. 
Compared to the MST, the CG method has a better predictive accuracy. However, the implementation of boundary conditions at predefined times is numerically different. The CG method does not allow for implementing prevailing boundary conditions, which is often applied in modelling pipeline failures such as ESDV closure at specified time and distance along the pipe. In contrast, the MST allows direct control on the time of input variables which are given at boundaries. As such, the MST is chosen for grid discretisation in this study.

\subsubsection{Numerical Formulation and Implementation of the Method of Characteristics}

The solution for PDEs using the MOC comprises two steps:

1. Conversion of the PDEs into a system of ODEs called the compatibility equations.

2. Solution of the compatibility relations based on the MST method employing the Euler predictor-corrector technique.

\subsubsection{Conversion of Partial Differential Equations to Ordinary Differential Equations}

According to Zucrow and Hoffman (1976), the governing conservation equations (PDEs) of mass, momentum and energy (see Section 2.3.2) can be replaced by three compatibility equations (ODEs). The following is a summary of the main results showing the final form of the compatibility relations and the characteristic curves (or simply characteristics) along which they hold.

The three compatibility relations associated with the conservation of mass, momentum and energy, which are valid along three characteristic equations, are respectively given as (Zucrow and Hoffman, 1976):

Path line compatibility equation:

$\rho_{o} d_{o} h-d_{o} P=\psi d_{o} t$ 
along the Path line characteristic $\left(\mathrm{C}_{0}\right)$ :

$$
\frac{d_{0} t}{d_{0} x}=\frac{1}{u}
$$

Positive Mach line compatibility equation:

$$
d_{+} P+\rho a d_{+} u=\left(a \alpha+\frac{\varphi \psi}{\rho T}\right) d_{+} t
$$

along the positive characteristic line (Mach line) $\left(\mathrm{C}_{+}\right)$:

$$
\frac{d_{+} t}{d_{+} x}=\frac{1}{u+a}
$$

Negative Mach line compatibility equation:

$$
\rho a d_{-} u-d_{-} P=\left(a \alpha-\frac{\varphi \psi}{\rho T}\right) d_{-} t
$$

along the negative characteristic line (Mach line) $\left(\mathrm{C}_{-}\right)$:

$$
\frac{d_{-} t}{d_{-} x}=\frac{1}{u-a}
$$

where, $t, x, u, a, P, \rho$ and $T$ are computed at each node point. The thermodynamic property, $\psi$ can be given by the following relation:

$$
\psi=\varphi \frac{Q_{h}-u \beta_{x}}{\rho T}
$$

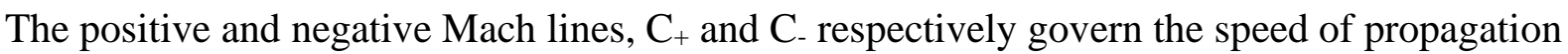
of the expansion and compression waves, while the Path line, $\mathrm{C}_{0}$ dictates the rate of flow through any given point along the pipeline. 


\subsubsection{Solution of the Compatibility Equations}

Figure 2.3 shows the schematic representation of Path line $\left(\mathrm{C}_{0}\right)$ and Mach lines $\left(\mathrm{C}_{+}, \mathrm{C}_{-}\right)$based on the MST grid construction. As mentioned previously, the solution for the compatibility equations necessitates the tracing of characteristic lines in a discretised $x$ - $t$ plane.

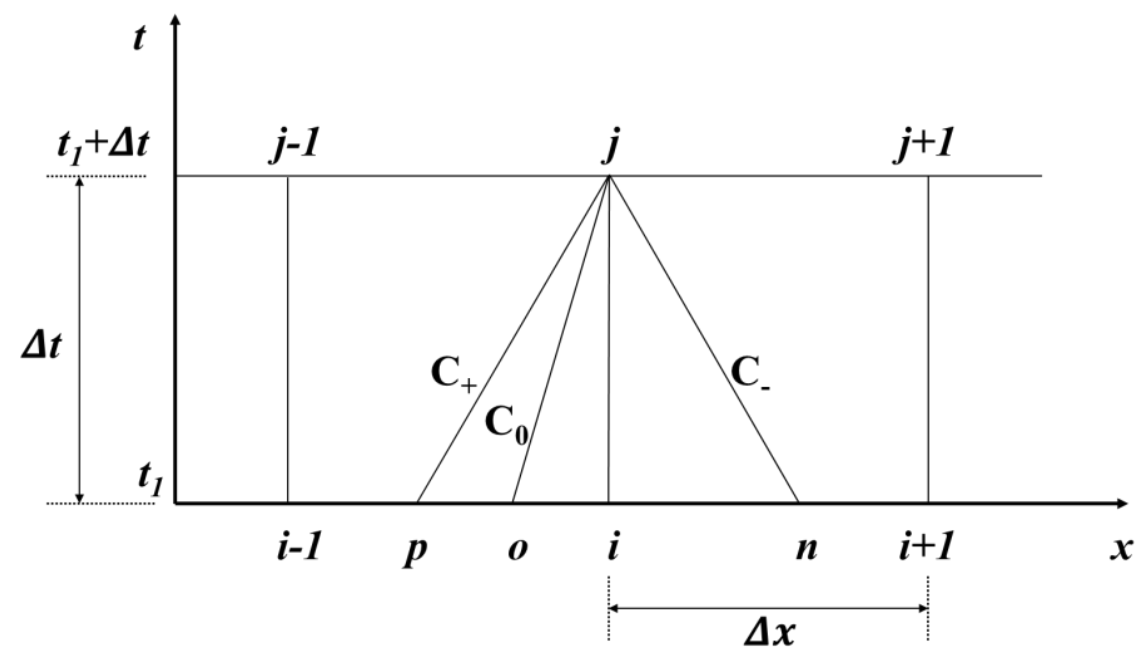

Figure 2.3: Schematic representation of Path line $\left(\mathrm{C}_{0}\right)$ and Mach lines $\left(\mathrm{C}_{+}, \mathrm{C}_{-}\right)$characteristics at a grid point along the time $(t)$ and space $(x)$ axes.

Here, at the initial time $t_{1}$, it is assumed that the fluid properties are already known at grid points $i-1, i$ and $i+1$. The initial conditions at the foot of each characteristic curve (i.e., $p$, $o$ and $n$ ) can be calculated by linear interpolation. A finite difference method is adopted to solve the compatibility equations to obtain the flow variables $P, h$ and $u$ at the intersection point $j$ at the next time step, $t_{1}+\Delta t$.

The Courant-Friedrichs-Lewy (CFL) criterion (Courant et al., 2002) is applied to calculate the pre-specified time. This criterion is a requirement for the stability of the numerical scheme employed for the system under consideration. It is given by:

$$
\Delta t \leq \frac{\Delta x}{|u+a|_{\max }}
$$

where, $\Delta t$ and $\Delta x$ are respectively the time step and distance interval. 


\subsubsection{Finite Difference Solution of Compatibility Equations}

Zucrow and Hoffman (1976) introduced the finite difference method used to resolve the compatibility relations. Following is a brief description highlighting the important aspects.

In order to solve the compatibility and characteristic equations (2.46 to 2.51 ) numerically, the Euler predictor-corrector finite difference technique is applied (Zucrow and Hoffman, 1976). The method consists of two steps: the explicit predictor step which is used as an estimate of the fluid properties at the solution point, followed by the corrector step which is applied as an initial estimate for an implicit approximation of the time step.

\subsubsection{First Order Approximation: Predictor Step}

In the predictor step the Path line, positive Mach line and negative Mach line compatibility equations (equations 2.46, 2.48 and 2.50) are respectively replaced with their finite difference form as (Atti, 2006):

$$
\begin{aligned}
& \rho_{o}\left(h_{j}-h_{o}\right)-\left(P_{j}-P_{o}\right)=\psi_{o}\left(t_{j}-t_{o}\right) \\
& (\rho a)_{p}\left(u_{j}-u_{p}\right)+\left(P_{j}-P_{p}\right)=\left(a \alpha+\frac{\varphi \psi}{\rho T}\right)_{p}\left(t_{j}-t_{p}\right) \\
& (\rho a)_{n}\left(u_{j}-u_{n}\right)-\left(P_{j}-P_{n}\right)=\left(a \alpha-\frac{\varphi \psi}{\rho T}\right)_{n}\left(t_{j}-t_{n}\right)
\end{aligned}
$$

where, the subscripts assigned to the various properties in equations 2.54 to 2.56 denote the location in space and time, as shown in figure 2.3. The locations $x_{p}, x_{o}$ and $x_{n}$ are determined from a first order finite difference form of equations $2.47,2.49$ and 2.51. The fluid properties from these locations are then linearly interpolated from those at grid points $i-1, i$ and $i+1$ (Atti, 2006). 


\subsubsection{Second Order Approximation: Corrector Step}

In the second step, the second order approximation is employed to improve the accuracy of the first order solution. The finite difference form of the Path line, positive Mach line and negative Mach line compatibility equations (equations 2.46, 2.48 and 2.50) can be respectively expressed as (Atti, 2006):

$$
\begin{aligned}
& \frac{1}{2}\left(\rho_{o}+\rho_{j}\right)\left(h_{j}-h_{o}\right)-\left(P_{j}-P_{o}\right)=\frac{1}{2}\left(\psi_{o}+\psi_{j}\right)\left(t_{j}-t_{o}\right) \\
& \frac{1}{2}\left[(\rho a)_{p}+(\rho a)_{j}\right]\left(u_{j}-u_{p}\right)+\left(P_{j}-P_{p}\right) \\
& =\frac{1}{2}\left[\left(a \alpha+\frac{\varphi \psi}{\rho T}\right)_{p}+\left(a \alpha+\frac{\varphi \psi}{\rho T}\right)_{j}\right]\left(t_{j}-t_{p}\right) \\
& \frac{1}{2}\left[(\rho a)_{n}+(\rho a)_{j}\right]\left(u_{j}-u_{n}\right)-\left(P_{j}-P_{n}\right) \\
& =\frac{1}{2}\left[\left(a \alpha-\frac{\varphi \psi}{\rho T}\right)_{n}+\left(a \alpha-\frac{\varphi \psi}{\rho T}\right)_{j}\right]\left(t_{j}-t_{n}\right)
\end{aligned}
$$

Similar to the predictor step, a second order finite difference form of equations 2.47, 2.49 and 2.51 is introduced to calculate the positions $x_{p}, x_{o}$ and $x_{n}$. The fluid properties at these points are then found by linear interpolation, as in the first order step. This calculation is repeated until a certain tolerance (ca. $10^{-5}$ ) is satisfied for the three independent flow variables: $P, h$ and $u$.

\subsection{Boundary Conditions for Simulating Pipeline Failure}

In this section, the mathematical formulation for defining the pertinent boundary conditions required for simulation of the outflow following pipeline failure are presented. Such boundary conditions include:

- Pipeline upstream conditions (closed end \& reservoir)

- Pipeline downstream conditions (closed end \& open end) 
- Full bore rupture (FBR)

- Puncture

- ESDV closure dynamic response

\subsubsection{Pipeline Upstream Conditions}

The grid scheme at the inlet end of the pipeline is presented in figure 2.4. Only Path line $\left(\mathrm{C}_{0}\right)$ and negative Mach line ( $\left.\mathrm{C}_{-}\right)$characteristics are applicable at the inlet end of the pipeline and hence only two compatibility equations are valid. Thus, the inlet end boundary condition must be applied to determine the pertinent flow variables.

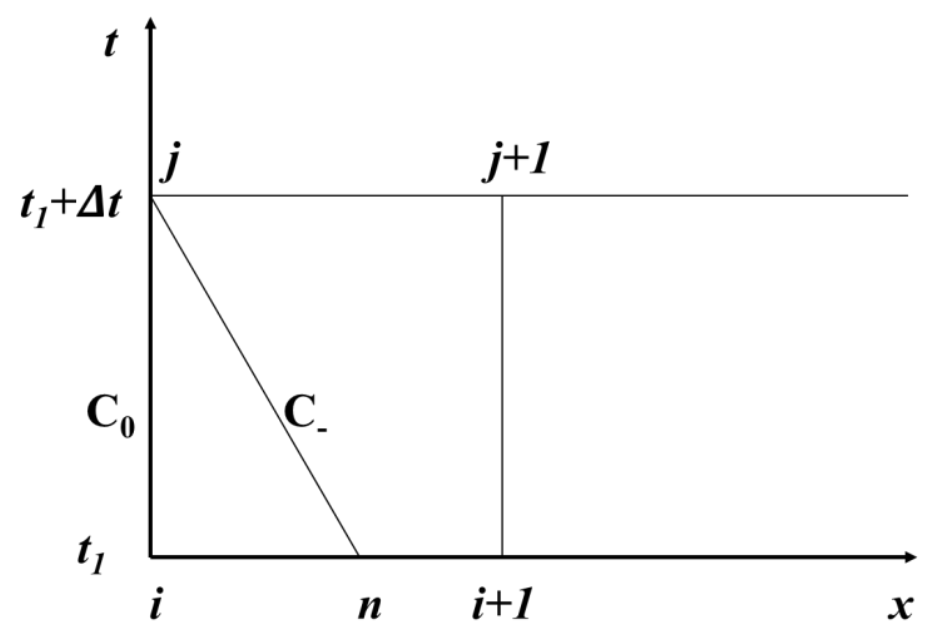

Figure 2.4: Schematic representation of the active characteristic lines $\left(\mathrm{C}_{0}\right.$ and $\left.\mathrm{C}_{\text {- }}\right)$ at the inlet end point along the time $(t)$ and space $(x)$ axes.

\subsubsection{Closed End}

For the closed end inlet boundary condition, the pipeline inlet flow velocity $\left(u_{j}\right)$ is zero. The first order finite difference approximation (equation 2.56) of negative Mach line compatibility equation $\left(\mathrm{C}_{-}\right)$is given as: 


$$
P_{j}=\left(\frac{\varphi \psi}{\rho T}-a \alpha\right)_{n} \Delta t-(\rho a u)_{n}+P_{n}
$$

Next, the upstream enthalpy $\left(h_{j}\right)$ can be updated via the solution of the first order finite difference approximation of the path line compatibility (equation 2.54) and is given as:

$$
h_{j}=\frac{\psi_{o} \Delta t+\left(P_{j}-P_{o}\right)+\rho_{o} h_{o}}{\rho_{o}}
$$

The corrector step as described in Section 2.4.3.2 is thereafter subsequently employed to yield the flow variables at the inlet closed end of the pipeline.

\subsubsection{Reservoir at Pipeline Inlet}

In the case of the upstream boundary condition corresponding to an infinite reservoir, the upstream pressure is assumed to remain constant and equal to the reservoir pressure $\left(P_{\text {res }}\right)$ despite pipeline failure. Thus, the pressure, $P_{j}$, at the solution point, $j$, at a given time, $t_{j}$, is fixed and equal to $P_{\text {res }}$.

By substituting $P_{j}=P_{\text {res }}$ into the negative Mach line compatibility equation (equation 2.56), the inlet flow velocity $\left(u_{j}\right)$ is given by (Oke, 2004):

$$
u_{j}=\frac{1}{(\rho a)_{n}} P_{r e s}-\frac{1}{(\rho a)_{n}}\left[\left(\frac{\varphi \psi}{\rho T}-a \alpha\right)_{n} \Delta t+P_{n}\right]+u_{n}
$$

Similarly, the enthalpy, $h_{j}$ can be obtained from the Path line compatibility equation (equation 2.54) via:

$$
h_{j}=\frac{\psi_{o} \Delta t+\rho_{o} h_{o}}{\rho_{o}}
$$

Next, the corrector step as described in Section 2.4.3.2 is subsequently applied. 


\subsubsection{Pipeline Downstream Conditions (Failure Plane)}

The pipeline downstream conditions at the failure plane are similar to the upstream conditions, where only two compatibility equations $\left(\mathrm{C}_{0}\right.$ and $\left.\mathrm{C}_{+}\right)$are valid. Figure 2.5 is the schematic representation of the grid scheme at the pipeline downstream failure plane.

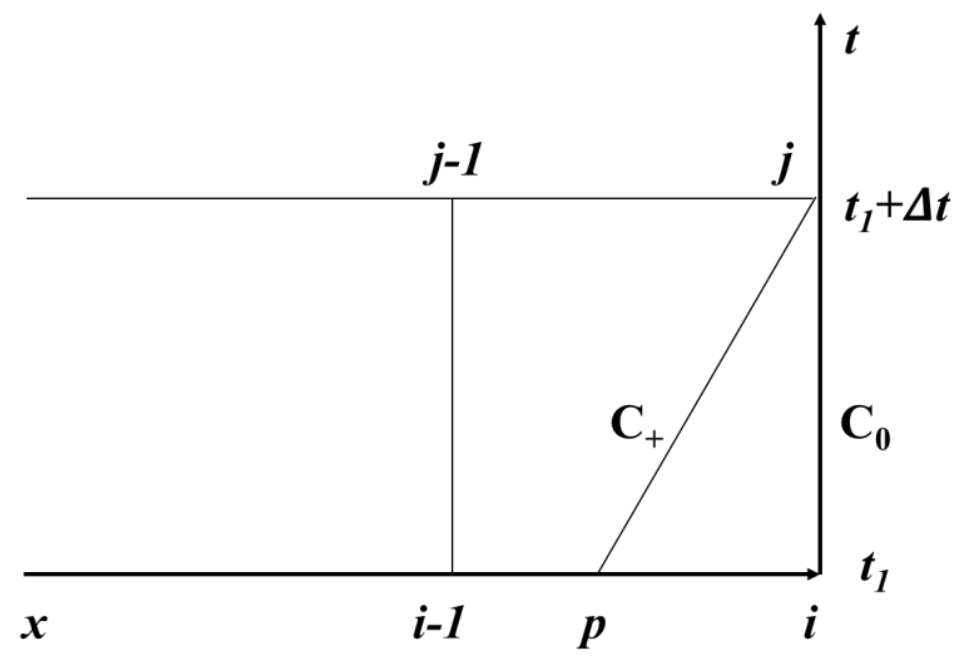

Figure 2.5: Schematic representation of the active characteristic lines $\left(C_{0}\right.$ and $\left.C_{+}\right)$at the pipeline downstream end point along the time $(t)$ and space $(x)$ axes.

\subsubsection{Closed End}

Given that the velocity at the closed end $\left(u_{j}\right)$ is zero, the first order finite difference approximation of the positive Mach line compatibility equation can be written as:

$$
P_{j}=\left(\frac{\varphi \psi}{\rho T}+a \alpha\right)_{p} \Delta t+(\rho a u)_{p}+P_{p}
$$

The upstream enthalpy can be calculated using the Path line compatibility equation:

$$
h_{j}=\frac{\psi_{o} \Delta t+\left(P_{j}-P_{o}\right)+\rho_{o} h_{o}}{\rho_{o}}
$$


Then, the corrector step as described in Section 2.4.3.2 is subsequently applied.

\subsubsection{Open Pipe End}

In such condition the pipe end is assumed to be open; that is the delivery point is the surrounding ambient. Here the outflow boundary condition is modelled using a simple zeroorder extrapolation method where all variables are equated to the corresponding values at the last numerical cell centre in the computational domain.

\subsubsection{Full Bore Rupture}

When a pipeline failure occurs, depending on the upstream and downstream pressures, the flow at the release plane may be either choked (critical) or unchoked.

In the case of choked flow, the fluid expands and discharges at a critical pressure (higher than the ambient pressure). Under this flow regime, the release flow rate is at a maximum and the release velocity corresponds to the sonic velocity at the prevailing pressure $\&$ temperature. As such, the conditions downstream of the release plane have no influence on the discharge process, which means no disturbance downstream of the release plane can propagate upstream.

Once the pressure at the release plane has reached the ambient pressure, the flow is said to be unchoked. In this case, the release velocity is subsonic, and the remaining discharged inventory is driven only by the momentum of the fluid.

Only the $\mathrm{C}_{0}$ and $\mathrm{C}_{+}$characteristics are applicable at the pipeline rupture plane. However, it is not possible to obtain the required fluid properties at the release plane in the absence of the $\mathrm{C}$. characteristic. To address this, a simple analytical relationship is employed to express the expansion process across the release plane. This involves the introduction of a 'ghost cell' at the fictitious node $(i+1)$ landing outside the rupture plane at the same time interval as node $i$ as shown in figure 2.6. 


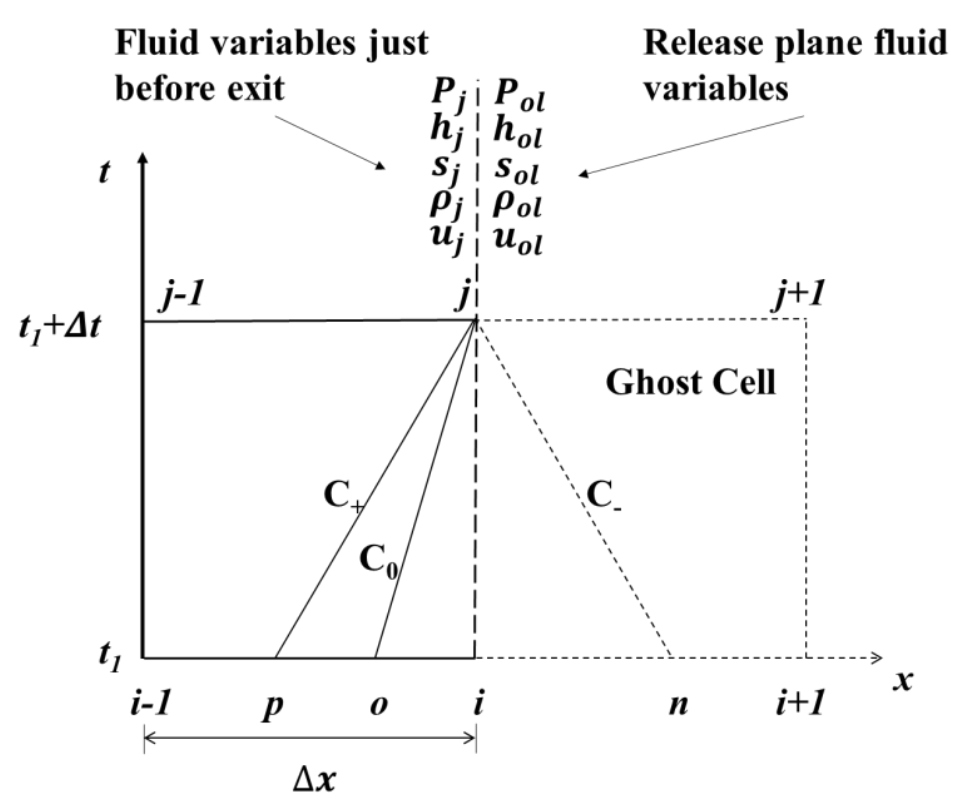

Figure 2.6: Schematic representation of the characteristic lines at the rupture plane based on the concept of a ghost cell along the time $(t)$ and space $(x)$ axes.

As can be observed from the figure, the introduction of the ghost cell allows the solution along the negative Mach line characteristic, $\mathrm{C}$.. The flow properties at point $j$ can be divided into two groups: the fluid variables just before exit (i.e. $P_{j}, h_{j}$ and $u_{j}$ ) and the release plane fluid variables (i.e. $P_{o l}, h_{o l}$ and $u_{o l}$ ). The first group of variables can be obtained in the same way as for interior points using equations 2.54 to 2.56. It is noteworthy that interpolation is not required within the ghost cell as all the properties within it are spatially invariant. For the second group of flow variables, the calculation is based on a discharge rate algorithm presented later in this section.

Figure 2.7 shows a schematic representation of the relevant pressures at the rupture plane governing the discharge rate in the case of a complete FBR. $P_{j}, P_{o l}$ and $P_{a m b}$ respectively represent the pressure of the fluid approaching the release plane (upstream), the discharge pressure and the ambient pressure (downstream). 


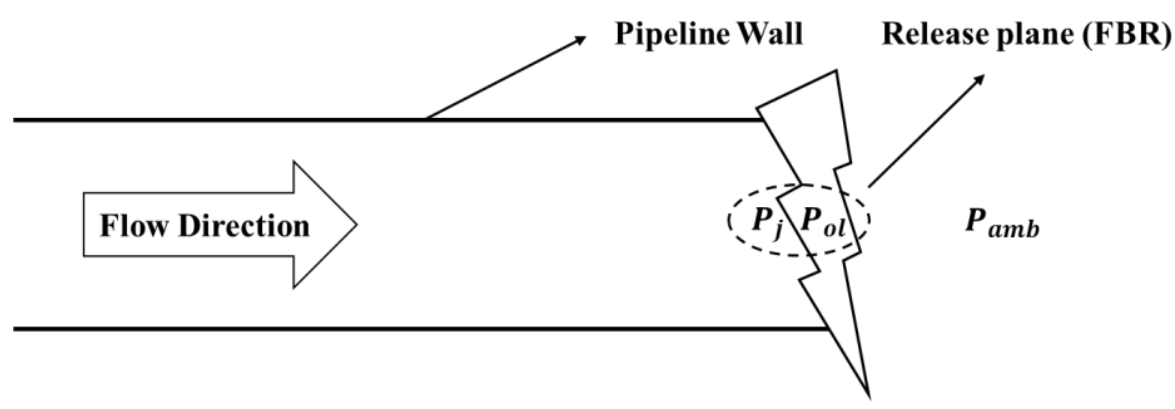

Figure 2.7: Schematic representation of pertinent pressures at the failure plane governing the discharge rate in the case a complete FBR

The calculation of the choked and unchoked flow velocities can be obtained through the application of the energy balance across the release plane. Ignoring changes in potential energy between the flow approaching and exiting the failure plane, the energy balance equation is given by:

$$
h_{j}+\frac{1}{2} u_{j}^{2}=h_{o l}+\frac{1}{2} u_{o l}^{2}
$$

Here, the subscripts $j$ and $o l$ respectively denote upstream and the release plane conditions.

In the case of choked flow, equation 2.66 is solved iteratively using the Brent iteration method (Brent, 1971) The velocity, $u_{o l}$ appearing in the equation, is replaced by the local speed of sound, $a_{o l}$ (i.e. $u_{o l}=a_{o l}$ ). The iterative solution of equation 2.66 involves guessing and updating the discharge pressure $\left(P_{o l}\right)$ in conjunction with the pressure-entropy (isentropic) flash calculation (in order to find $h_{o l}$ and $a_{o l}$ ) until equation 2.66 is satisfied. Other flow variables at the rupture plane (e.g. $\rho_{o l}, T_{o l}$ ) can then be determined from a pressure-entropy flash calculation once a solution is obtained.

Turning to unchoked flow, as mentioned earlier, the release pressure $\left(P_{o l}\right)$ is equal to the ambient pressure $\left(P_{a m b}\right.$; see figure 2.7). Thus, the release enthalpy $\left(h_{o l}\right)$ is determined from a pressure-entropy flash calculation $\left(P=P_{a m b}, s=s_{j}\right)$. Then substituting $h_{o l}$ in equation 2.66, the release velocity $\left(u_{o l}\right)$ is obtained. Unlike in the case of choked flow, under this condition no iteration is required in determining flow conditions at the rupture plane. 
Following equation 2.66, $u_{j}$ is updated once the release plane flow conditions are determined. The updated flow variables (i.e. $P_{j}, h_{j}$ and $u_{j}$ ) are thereafter employed in the corrector step (see Section 2.4.3.2) until convergence is observed. The calculation algorithm for determining flow variables at the failure plane is shown in figure 2.8 .

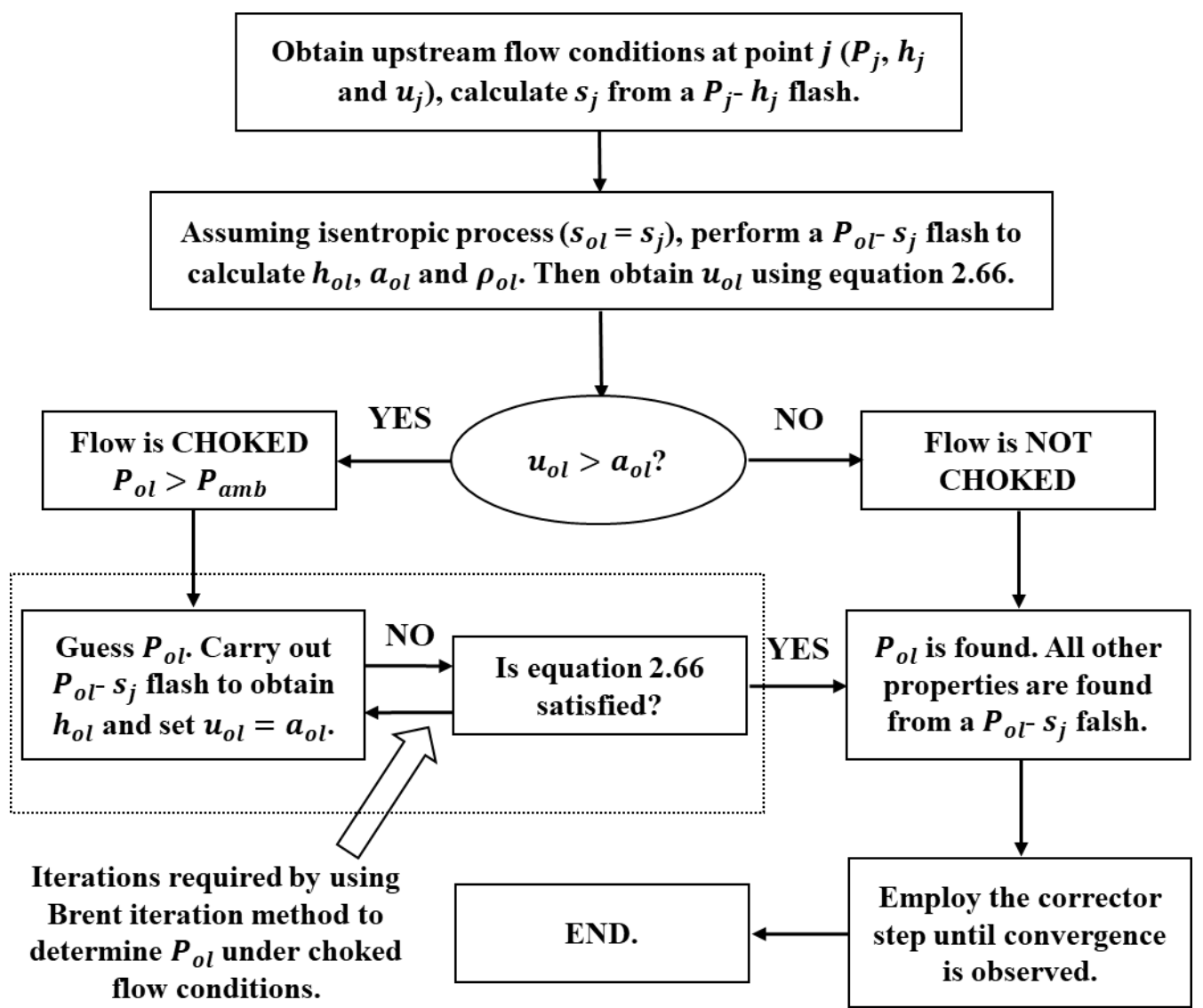

Figure 2.8: Calculation algorithm for determining flow variables at the failure plane.

\subsubsection{Pipeline Puncture}

Oke et al. (2003) introduced the boundary condition for the puncture along a pipeline. The method was later adjusted by Atti (2006) to correct the invalid constant density assumption across the release plane. In this study, Atti's (2006) puncture boundary is therefore employed. Figure 2.9 is a schematic representation of the fluid flow process following the occurrence of a puncture along the pipe wall. 


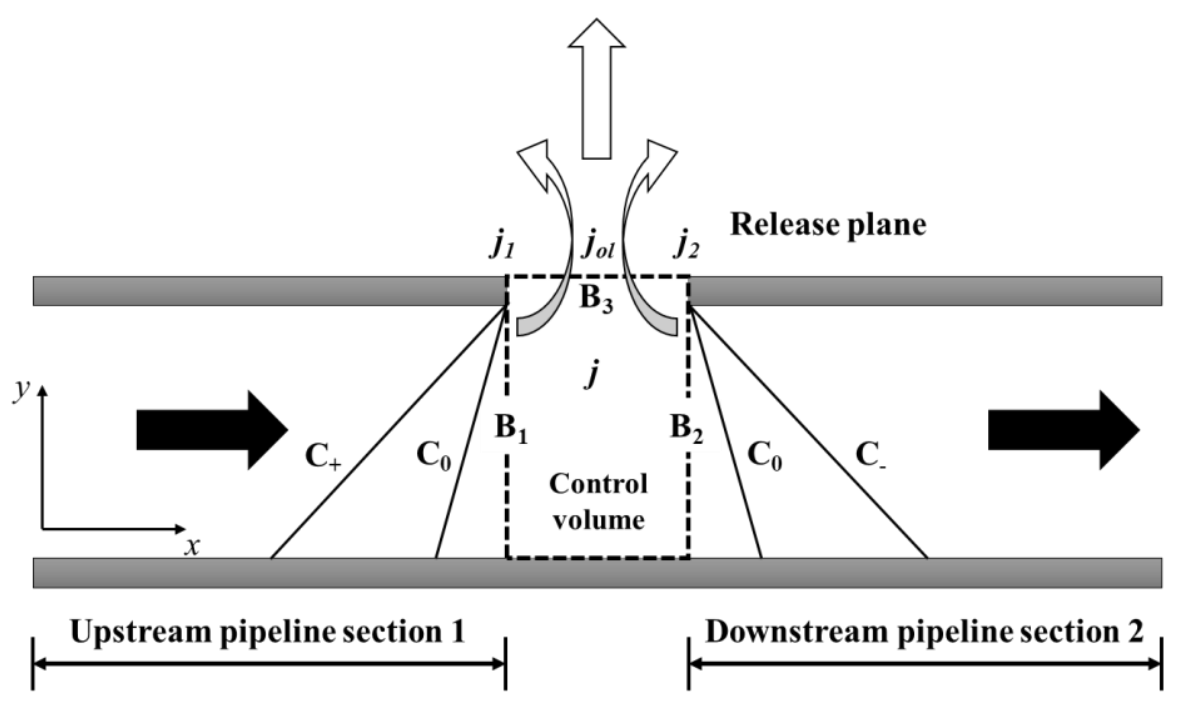

Figure 2.9: Schematic representation of fluid flow analysis following pipeline puncture along the pipeline length.

As can be observed in figure 2.9, the pipeline is split at the puncture point into two parts: upstream pipe section 1 and downstream pipe section 2. Also, the puncture point is treated as a common junction between the two pipeline sections. Pipeline sections 1 and 2 terminate and emanate from the puncture junction, respectively. As a result, there are three flow boundaries $\left(\mathrm{B}_{1}, \mathrm{~B}_{2}\right.$, and $\mathrm{B}_{3}$ in figure 2.9 , denoted by $j_{1}, j_{2}$ and $j_{\text {ol }}$ respectively) at the common junction with each requiring the imposition of appropriate boundary conditions.

It should be noted that for upstream and downstream of the common junction (pipeline section 1 , i.e., plane $\mathrm{B}_{1}$ and pipeline section 2 , i.e., plane $\mathrm{B}_{2}$ ), only two compatibility equations are applicable. A control volume, which is fixed in time and space, is introduced in the modelling to bound boundaries $\mathrm{B}_{1}$ to $\mathrm{B}_{3}$.

Assuming the pressure in the control volume is constant at the current time step, which gives:

$$
P_{j_{1}}=P_{j_{2}}=P_{j}
$$

where, $P_{j_{1}}, P_{j_{2}}$ and $P_{j}$ are respectively the pressure at $j_{1}, j_{2}$ and within the control volume. Defining the fluid properties in the control volume at the cell centre gives: 


$$
s_{j}=\frac{s_{j_{1}}+s_{j_{2}}}{2}
$$

The flow through the release plane $\left(\mathrm{B}_{3}\right)$ is assumed to be isentropic, hence:

$$
s_{o l}=s_{j}
$$

As may be observed in the figure 2.9, the flow through the control volume is two-dimensional. The mass conservation equation to account for both in radial and axial direction is therefore given by:

$$
V \frac{d \rho}{d t}+A_{\text {pipe }} \partial(\rho u)+A_{o l} \partial(\rho v)=0
$$

where, $V$ is the volume of the control volume. $v$ is the fluid velocity normal to the axial direction (i.e. in $y$ direction).

Next, integration of equation 2.70 with respect to time, $t$, over the interval $\left[t_{1}, t_{2}\right]\left(\Delta t=t_{2}-\right.$ $\left.t_{1}\right)$ gives:

$$
\begin{gathered}
V\left(\rho_{j \mid t=t_{2}}-\rho_{j \mid t=t_{1}}\right)+A_{\text {pipe }} \int_{t_{1}}^{t_{2}}\left[(\rho u)_{x_{2}}-(\rho u)_{x_{1}}\right] d t \\
+A_{o l} \int_{t_{1}}^{t_{2}}\left[(\rho v)_{y_{2}}-(\rho v)_{y_{1}}\right] d t=0
\end{gathered}
$$

In the case of FBR, given the almost instantaneous large pressure drop at the rupture plane, the expansion process at the release plane is assumed to be isentropic and the mass flow rate is conserved across the release plane (no accumulation of mass is assumed to occur). However, in the case of puncture, non-isentropic effects may occur due to the hydraulic resistance posed by the release orifice. Consequently, the actual mass flow rate of the exiting fluid at the release plane will be smaller than the isentropic mass flow rate. Therefore, the discharge coefficient, $C_{d}$, is introduced to express the ratio between these two mass flow rates (Atti, 2006): 


$$
C_{d}=\frac{\rho_{j} u_{i} A_{\text {pipe }}}{\rho_{\text {ol }} u_{o l} A_{o l}}
$$

where, $A_{\text {pipe }}$ and $A_{o l}$ are the area of the pipe and orifice, respectively.

Given that $(\rho v)_{y_{1}}=0$, multiplying the discharge coefficient, $C_{d}$ (equation 2.72), on the release through the orifice to account for non-isentropic effects and numerically evaluating the integrals, equation 2.71 becomes:

$$
\begin{aligned}
V\left(\rho_{j \mid t=t_{2}}-\rho_{j \mid t=t_{1}}\right) & \\
& +\left\{A_{\text {pipe }}\left[(\rho u)_{j \mid x=x_{2}}-(\rho u)_{j \mid x=x_{1}}\right]+C_{d} A_{o l}\left((\rho v)_{j \mid y=y_{2}}\right)_{a v e}\right\} \Delta t \\
& =0
\end{aligned}
$$

where, the subscript ave represents the average of the value in the brackets in the interval $\left[t_{1}, t_{2}\right] . x_{1}$ and $x_{2}$ represent the lower $\left(\mathrm{B}_{1}\right)$ and upper $\left(\mathrm{B}_{2}\right)$ boundaries along the $\mathrm{x}$-axis of the control volume $V$.

In order to obtain a solution at the common junction, equation 2.73 , which represents the boundary equation based on the two-dimensional mass conservation coupling the flow behaviour at planes $B_{1}$ to $B_{3}$, must be simultaneously satisfied. Figure 2.10 shows the calculation algorithm for determining fluid flow transport properties at the release plane for puncture along the pipeline length. 


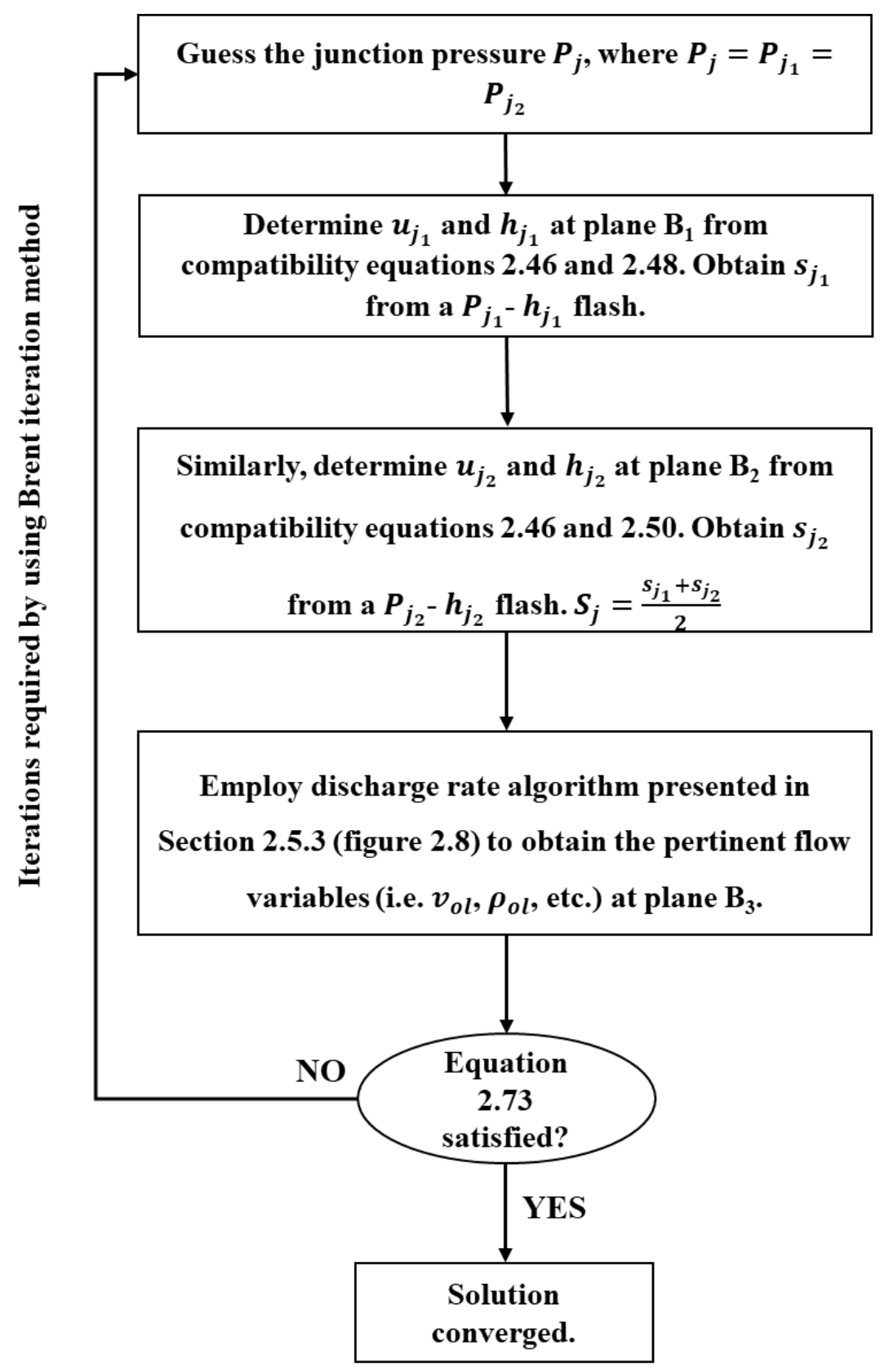

Figure 2.10: Calculation algorithm for determining pressure and other pertinent flow variables at the release plane for puncture along the pipeline length.

\subsubsection{Emergency Shutdown Valve Closure Modelling}

The following presents the development of the relevant boundary conditions (closure equations) for inline Check Valves (CVs), Automatic Shut-off Valves (ASVs) and Remote 
Control Valves (RCVs) in order to determine their impact on the transient fluid flow behaviour following pipeline failure.

\subsubsection{Check Valves (CVs)}

CVs (also known as non-return valves) are placed in pipelines to prevent back-flow following a pipeline failure. Ideally, a check valve closes instantaneously preventing back-flow when flow reversal occurs at the location of the valve. However, in practice, the closure occurs after some level of back-flow is established. In both cases, a pressure surge (increase) is expected upon value closure.

$\mathrm{CV}$ closure is modelled by introducing closed end boundary conditions (Sections 2.5.1.1 and 2.5.2.1) at the required time and space co-ordinates (Saha, 1997). A worst-case scenario assumption corresponding flow being unhindered until complete closure of the valve is made.

Figure 2.11 shows the schematic representation of the appropriate boundary conditions on either side of a check valve. It should be noted that, the $\mathrm{C}_{0}$ characteristic on either side is zero since the flow velocity at that point is zero.

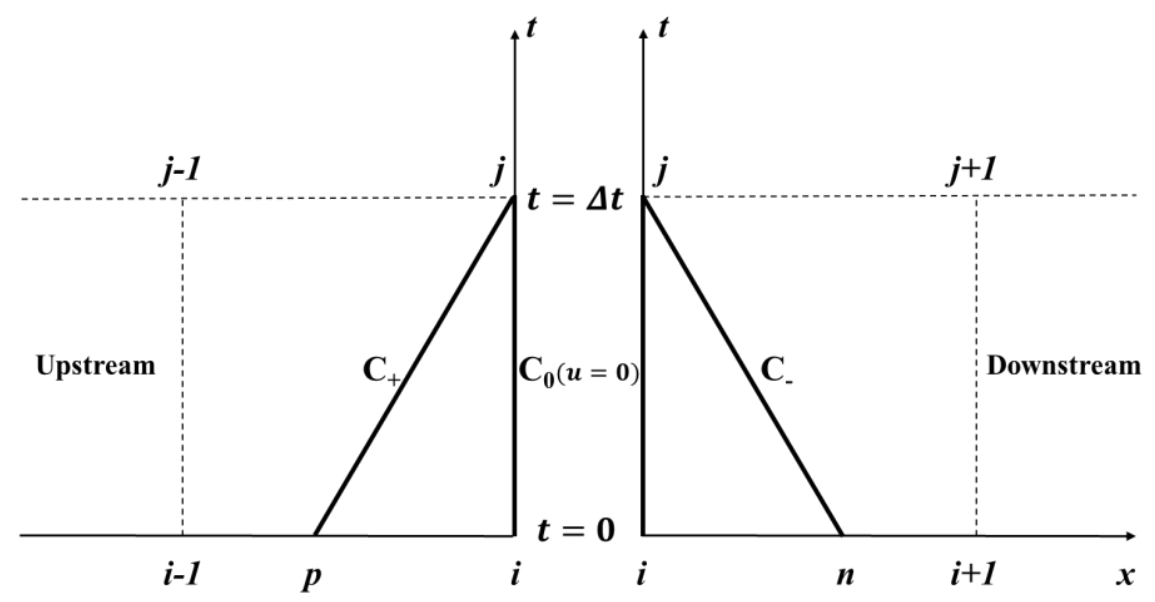

Figure 2.11: Schematic representation of the active characteristic lines at either side of a check valve along the time $(\mathrm{t})$ and space $(\mathrm{x})$ axes. 
Hence, the solution pressure, $P_{j}$, on the downstream and upstream sides can be respectively obtained by equations 2.60 and 2.64 .

\subsubsection{Automatic Shut-off Valves (ASVs) and Remote Control Valves (RCVs)}

The closure for both ASVs and RCVs are assumed to occur during the time frame between the valve activation time, $t_{a}$ and its complete closure time, $t_{c}$. In the case of ASVs, the valve activation time, $t_{a}(\mathrm{ASV})$ corresponds to the time when the fluid pressure drops below a set valve activation pressure, $\Delta p_{a}$. Hence, $t_{a}$ (ASV) depends on the fluid decompression wave velocity and the distance between the pipe failure location and the valve.

$\mathrm{RCV}$ activation time, $t_{a}(\mathrm{RCV})$ on the other hand, is defined as the sum of the time lapse for detection of the pressure drop at the valve location (here equals to $t_{a}(\mathrm{ASV})$ ) and the operator response/action time to activate valve closure.

In order to account for the closure of ASVs and RCVs, specific boundary conditions are required given its relatively complicated closure geometry and slow closure rate. Hence, the variation of fluid flow properties must be calculated as a function of time during valve closure.

Accordingly, following Mahgerefteh et al. (1997), the compatibility equations need to be rearranged to take account of the pressure drop across the closing valve. Figure 2.12 presents the schematic representation of the characteristic lines across a ASV or RCV along the time (t) and space $(\mathrm{x})$ axes. 


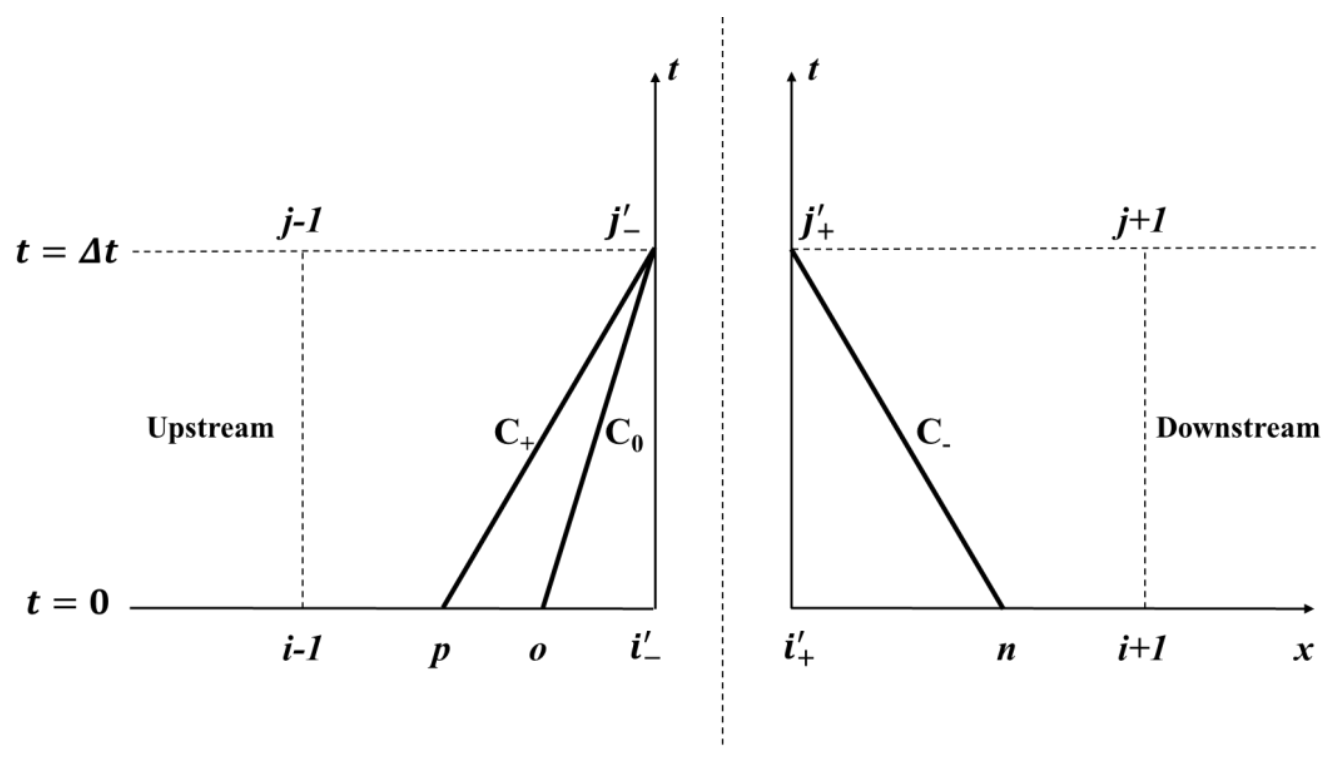

Figure 2.12: Schematic representation of the active characteristic lines at either side of a ASV or RCV along the time (t) and space (x) axes.

As it may be observed from the figure, the nodes across the valve (upstream and downstream sides) are respectively denoted by $j_{-}^{\prime}$ and $j_{+}^{\prime}$. The Path line, positive Mach line and negative Mach line compatibility equations are respectively given by:

$$
\begin{aligned}
& P_{j_{+}^{\prime}}-P_{o}-\left(\overline{a^{2}}\right)_{o j_{+}^{\prime}}\left(\rho_{j}-\rho_{o}\right)=(\bar{\psi})_{o j_{+}^{\prime}} \Delta t=K_{4} \\
& P_{j_{+}^{\prime}}-P_{p}+(\overline{\rho a})_{p j_{+}^{\prime}}\left(u_{j}-u_{p}\right)=(\bar{\psi}+\overline{a \beta})_{p{j_{+}^{\prime}}^{\prime}} \Delta t=K_{5} \\
& P_{j_{-}^{\prime}}-P_{n}-(\overline{\rho a})_{n j_{-}^{\prime}}\left(u_{j}-u_{n}\right)=(\bar{\psi}-\overline{a \beta})_{n j_{-}^{\prime}} \Delta t=K_{6}
\end{aligned}
$$

In equations 2.74 to 2.76 , four variables change with time, namely $P_{j_{+}^{\prime}}, P_{j_{-}^{\prime}}, u_{j}$ and $\rho_{j}$. In order to solve the above compatibility equations, the closure relation can be introduced using the valve loss equation (Swaffied and Boldy, 1993; Wylie et al., 1993):

$$
Q(t)=C_{d}(t) A_{f}(t) \sqrt{\frac{2 \Delta P(t)}{\rho(t)}}
$$


where, $Q$ and $A_{f}$ are respectively the volumetric flow rate and the area of flow through the valve at any time, $t$, during closure. The valve discharge coefficient, $C_{d}$, is a function of valve type and degree of opening. The Wylie's correlation (Wylie and Streeter, 1978) is applied in this study for a ASV or RCV:

$$
C_{d}=A_{0}+A_{1} \omega+A_{2} \omega^{2}+A_{3} \omega^{3}+A_{4} \omega^{4}
$$

where, $\omega$ represents the percentage of the valve opening area. $A_{0}, A_{1}, A_{2}, A_{3}$ and $A_{4}$ are curve fitting constants and their values are given by: $A_{0}=-0.00111888, A_{1}=0.001104507, A_{2}=$ $8.13 \mathrm{E}-05, A_{3}=-1.73 \mathrm{E}-06$ and $A_{4}=1.81 \mathrm{E}-08$.

The valve opening area to flow can be calculated as a function of the speed of closure of the valve. The ASV and RCV closure geometry is demonstrated in figure 2.13.

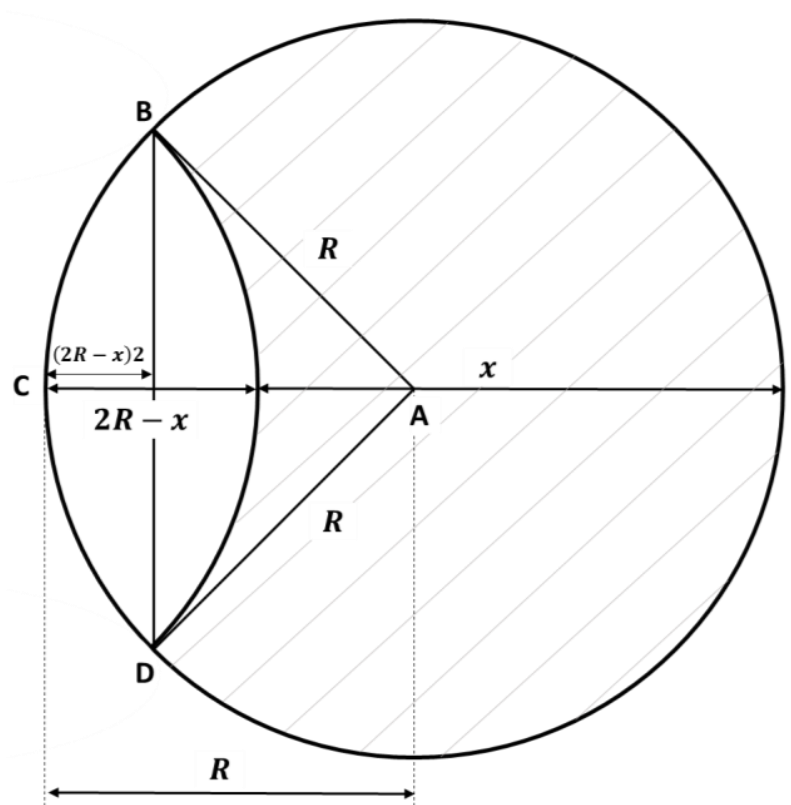

Figure 2.13: Schematic representation of ASV and RCV closure geometry (Mahgerefteh et al., 1997).

Referring to figure 2.13 , the unshaded area represents the valve flow area, $A_{f}$, whereas the shaded area is the area covered by the valve. $A_{f}$ can be calculated as twice of the area, $\mathrm{BCD}$, which is obtained by subtracting the area of the triangle area ABD from the area of the sector, ABCD. Hence, $A_{f}$ is given by (Mahgerefteh et al., 1997): 


$$
A_{f}=2\left[\pi R^{2} \frac{2 \cos ^{-1}\left(\frac{R-\left(\frac{2 R-x(t)}{2}\right)}{R}\right)}{360}-\left(R-\frac{(2 R-x(t))}{2}\right)\left(\sqrt{R^{2}-\left(R-\frac{(2 R-x(t))}{2}\right)^{2}}\right)\right]
$$

Here, $R$ is the pipeline radius. $x$, is the distance traversed by valve at time $t$, given by:

$$
x=u_{v} \times t
$$

where, $u_{v}$ is the valve linear closure rate.

The corresponding volumetric flow rate through the valve can be also expressed as a function of the fluid velocity, $u(t)$ as:

$$
Q(t)=u(t) A_{f}(t)
$$

Substituting equation 2.81 into equation 2.77 gives:

$$
u(t)=C_{d}(t) \sqrt{\frac{2 \Delta P(t)}{\rho(t)}}
$$

Taking ratios of flow rate over a given time interval during closure, the pressure loss across the closing valve is given by:

$$
\Delta P(t)=P_{j_{+}^{\prime}}-P_{j_{-}^{\prime}}=\frac{\Delta P_{0}}{u_{0}^{2} C_{d}^{2} \rho_{0}} u_{j}^{2} \rho_{j}=K_{7} u_{j}^{2} \rho_{j}
$$

where,

$$
K_{7}=\frac{\Delta P_{0}}{u_{0}^{2} C_{d}^{2} \rho_{0}}
$$


$\Delta P_{0}$ is the pressure loss when a valve is fully opened over the pipe element that contains the valve $\left(P_{i-1}-P_{i+1}\right.$, as depicted in figure 2.12). $u_{0}$ and $\rho_{0}$ are respectively the fluid velocity and density through the fully opened valve.

The pressure loss across the valve, $\Delta P(t)$ can also be obtained from the compatibility equations 2.74 to 2.76 . Subtracting equation 2.76 from equation 2.74 gives:

$$
\begin{aligned}
P_{j_{+}^{\prime}}-P_{j_{-}^{\prime}}= & K_{4}-K_{6}+P_{o}-P_{n}-u_{j}(\overline{\rho a})_{n j_{-}^{\prime}}+u_{n}(\overline{\rho a})_{n j_{-}^{\prime}}+\left(\overline{a^{2}}\right)_{o j_{+}^{\prime}} \rho_{j} \\
& -\left(\overline{a^{2}}\right)_{o j_{+}^{\prime}} \rho_{o}
\end{aligned}
$$

Similarly, from equations 2.75 and 2.76 gives:

$$
\begin{aligned}
& P_{j_{+}^{\prime}}-P_{j_{-}^{\prime}}=K_{5}-K_{6}+P_{p}-P_{n}-u_{j}\left[(\overline{\rho a})_{p j_{+}^{\prime}}+(\overline{\rho a})_{n j_{-}^{\prime}}\right]+(\overline{\rho a})_{p j_{+}^{\prime}} u_{p} \\
& +(\overline{\rho a})_{n j_{-}^{\prime}} u_{n}
\end{aligned}
$$

Equating equations 2.85 and 2.86 and rearranging gives:

$$
\rho_{j}=\frac{K_{8}-u_{j}(\overline{\rho a})_{p j_{+}^{\prime}}}{\left(\overline{a^{2}}\right)_{o j_{+}^{\prime}}}
$$

where,

$$
K_{8}=K_{5}-K_{4}+P_{p}-P_{o}+(\overline{\rho a})_{p j_{+}^{\prime}} u_{p}+\left(\overline{a^{2}}\right)_{o j_{+}^{\prime}} \rho_{o}
$$

Substituting $\rho_{j}$ from equation 2.88 into equation 2.83 gives:

$$
P_{j_{+}^{\prime}}-P_{j_{-}^{\prime}}=K_{7} u_{j}^{2} \rho_{j}=K_{7} u_{j}^{2}\left[\frac{K_{8}-u_{j}(\overline{\rho a})_{p j_{+}^{\prime}}}{\left(\overline{a^{2}}\right)_{o j_{+}^{\prime}}}\right]
$$

Equating equation 2.89 to equation 2.86 , the fluid velocity, $u_{j}$ is obtained from the following cubic equation: 


$$
u_{j}^{3}+K_{9} u_{j}^{2}+K_{10} u_{j}+K_{11}=0
$$

where,

$$
\begin{aligned}
& K_{9}=\frac{-K_{8}}{(\overline{\rho a})_{p j_{+}^{\prime}}} \\
& K_{10}=\frac{\left[(\overline{\rho a})_{p j_{+}^{\prime}}+(\overline{\rho a})_{n j_{-}^{\prime}}\right]\left(\overline{a^{2}}\right)_{o j_{+}^{\prime}}}{(\overline{\rho a})_{p j_{+}^{\prime}} K_{7}} \\
& K_{11}=\left[K_{5}-K_{6}+P_{p}-P_{n}+(\overline{\rho a})_{p j_{+}^{\prime}} u_{p}+(\overline{\rho a})_{n j_{-}^{\prime}} u_{n}\right] \frac{\left(\overline{a^{2}}\right)_{o j_{+}^{\prime}}}{(\overline{\rho a})_{p j_{+}^{\prime}} K_{7}}
\end{aligned}
$$

Three real roots can be obtained from equation 2.90, where two of them are positive. The solution can be selected by the root which is less than the maximum choke velocity at the rupture plane. The remaining dependent variables (i.e. $P_{j_{-}^{\prime}}, P_{j_{+}^{\prime}}$ and $\rho_{j}$ ) are calculated from the compatibility equations 2.74 to 2.76 .

After full valve closure, the $j_{-}^{\prime}$ and $j_{+}^{\prime}$ node points are assumed to be closed end node points, where the velocities $u_{j_{-}^{\prime}}$ and $u_{j_{+}^{\prime}}$ are both assumed to be 0 . The compatibility relations are then solved simultaneously as discussed in Sections 2.5.1.1 and 2.5.2.1 for the closed end boundary condition.

\subsection{Applications of the HEM Model}

In the following, a number of examples of the HEM model applications in simulating pipeline outflow during decompression are presented. In particular, the evaluation of the model performance is discussed based on the degree of agreement with experimental data for pipeline decompression tests $\&$ field results. 


\subsubsection{Mahgerefteh et al. (1999)}

\subsubsection{Model Introduction}

The pipeline decompression model developed by Mahgerefteh et al. (1999) is a robust computational fluid dynamic tool which is the most validated among its kind reported in open literature. The model was originally developed based on the HEM assumption for the quantitative failure consequence assessment in the event of high-pressure hydrocarbon and $\mathrm{CO}_{2}$ pipeline Full Bore Rupture (FBR) failures (Mahgerefteh et al., 1999, 2007, 2008). Moody friction factor (Massey and Ward-Smith, 1998) was employed to determine the fluid/wall drag interaction. Heat transfer across the pipe wall to the fluid followed a liner behaviour given by Newton's cooling law. The PR EoS (Peng and Robinson, 1976) was employed to calculate the fluid thermophysical properties and phase equilibrium data. The Method of Characteristics (MOC) was used as the mathematical solution of the model.

Oke et al. (2003) extended the flow model for predicting outflow following the puncture failures of pressurised pipelines. In their subsequence publications, other important features were performed, including the dynamic response of ESDVs (Mahgerefteh et al., 1997, 2000), the development of a simplified analytically based vessel blowdown model (VBM) (Mahgerefteh et al., 2011), decompression of pipeline networks (Mahgerefteh et al., 2006) and ductile fracture propagation of the pipe wall (Mahgerefteh et al., 2010, 2012).

In the next section, the model validations are presented followed by the review of the development of the dynamic response simulation of ESDVs.

\subsubsection{Model Validations}

The HEM flow model has been validated against multiple experimental data obtained from large-scale high-pressure pipeline decompression experiments for both hydrocarbons and $\mathrm{CO}_{2}$. A selection of the relevant validation tests are presented in the following. 


\section{Isle of Grain LPG Depressurisation Tests P40 \& P45 (Richardson and Saville, 1994)}

The HEM model has been validated against the experimental data which was conducted jointly by Shell Oil and BP at the Isle of Grain (Richardson and Saville, 1994). A series of pipeline depressurisation tests were performed simulating both FBR and puncture failures. The tests were based on the depressurisation of an instrumented $100 \mathrm{~m}$ long and $0.154 \mathrm{~m}$ diameter pipeline containing commercial LPG (95 mol\% propane, $5 \mathrm{~mol} \% \mathrm{n}$-butane). The pipeline roughness and thickness were respectively $0.05 \mathrm{~mm}$ and $7.3 \mathrm{~mm}$. In the experiment, the pipeline was ruptured using a bursting disc placed at one of its closed ends. The following are the model validation results for Isle of Grain LPG depressurisation tests P40 (FBR) and P45 (puncture).

The initial pipeline pressure and temperature for the Isle of Grain test P40 were respectively at 21.6 bar and $20{ }^{\circ} \mathrm{C}$. The ambient temperature was $19.1{ }^{\circ} \mathrm{C}$. Figures 2.14 to 2.16 respectively show the comparison of the simulated pressure, temperature and inventory variations with time against the Isle of Grain P40 measured data. The pressure and temperature data include both the closed and open ends of the pipeline following a FBR at its end.

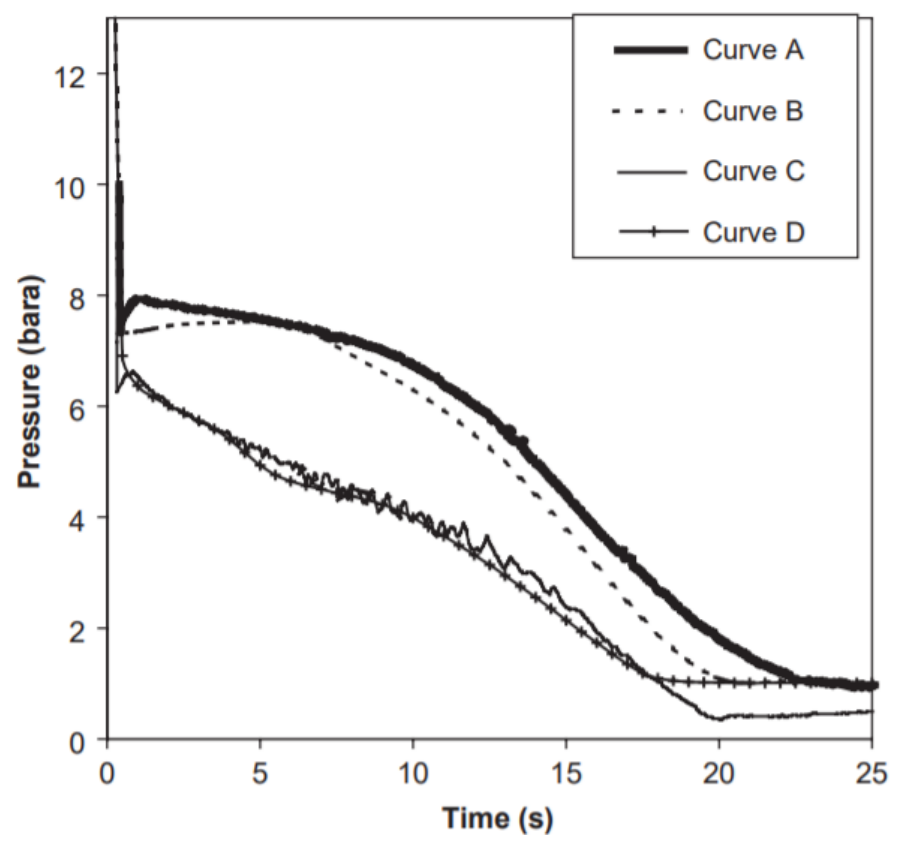

Figure 2.14: Pressure-time profiles at closed and open ends for the Isle of Grain P40 test (LPG). Curve A: field data (closed end); Curve B: model predictions (closed end); Curve C: field data (open end); Curve D: model predictions (open end) (Oke et al., 2003). 


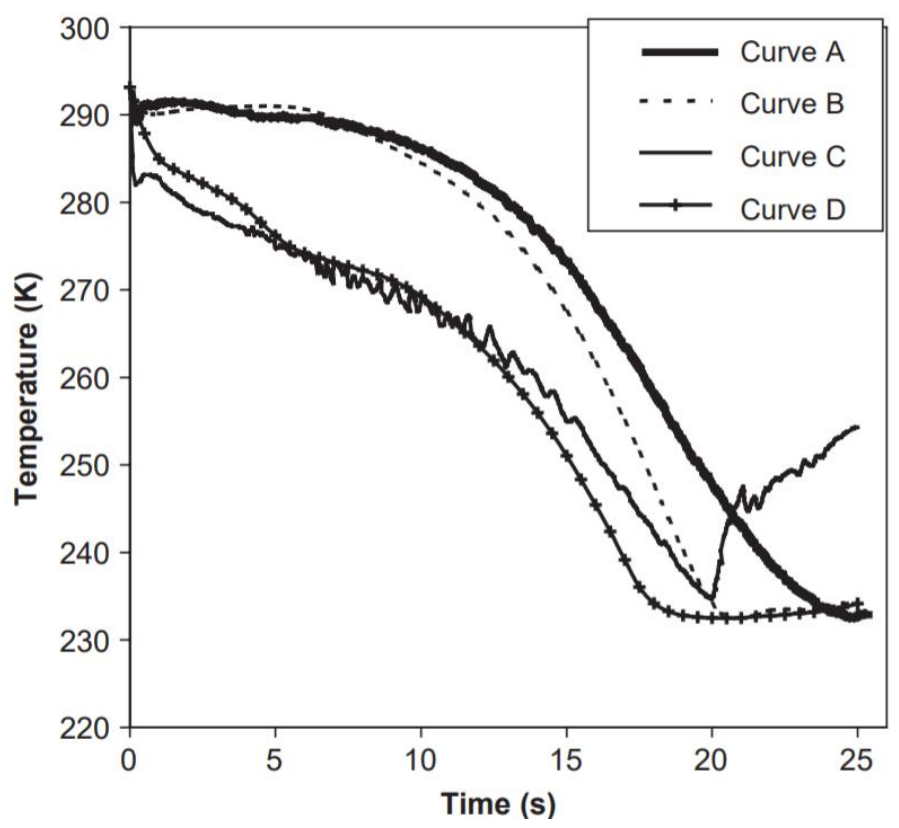

Figure 2.15: Temperature-time profiles at closed and open ends for the Isle of Grain P40 test (LPG). Curve A: field data (closed end); Curve B: model predictions (closed end); Curve C: field data (open end); Curve D: model predictions (open end) (Oke et al., 2003).

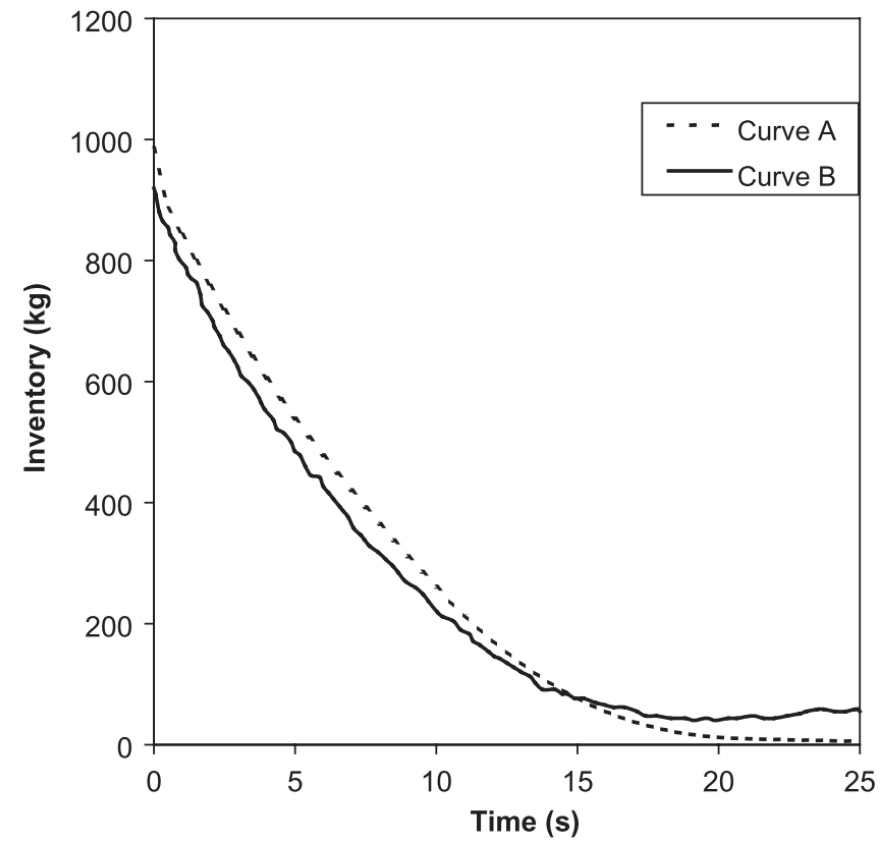

Figure 2.16: Total line inventory predictions for the Isle of Grain P40 test (LPG). Curve A: field data; Curve B: model predictions (Oke et al., 2003).

As can be observed from figures 2.14 to 2.16 , the flow model reasonably accurately predicts the initial rapid drop in the line pressure which is as a result of the phase change from liquid to 
two-phase. However, in figure 2.15, a rapid rise of temperature towards the end of depressurisation at the open end of the pipe can be seen from the field data. The rise in temperature corresponds to the transition from two-phase to gas flow and is a consequence of the different fluid/wall heat transfer coefficients. According to the authors, such observation was due to the constant heat transfer coefficient assumed for the simulation, thus the rise in the temperature was not reflected in the simulation data (Oke et al., 2003). With regards to the inventory variations with time, the amount decreases monotonically as expected. In general, the model's predictions agree reasonably well with measured data.

The Isle of Grain P45 test consisted of the pipeline depressurisation following a puncture $(75 \mathrm{~mm})$ failure at the end of the pipeline. The initial pressure, temperature and ambient temperature were at 11.4 bar, $15.9{ }^{\circ} \mathrm{C}$ and $16.7{ }^{\circ} \mathrm{C}$, respectively. Figure 2.17 shows the comparison of the simulated pressure as a function of time against the Isle of Grain P45 measured data. As it may be observed, the model predictions are in good accord with the field data.

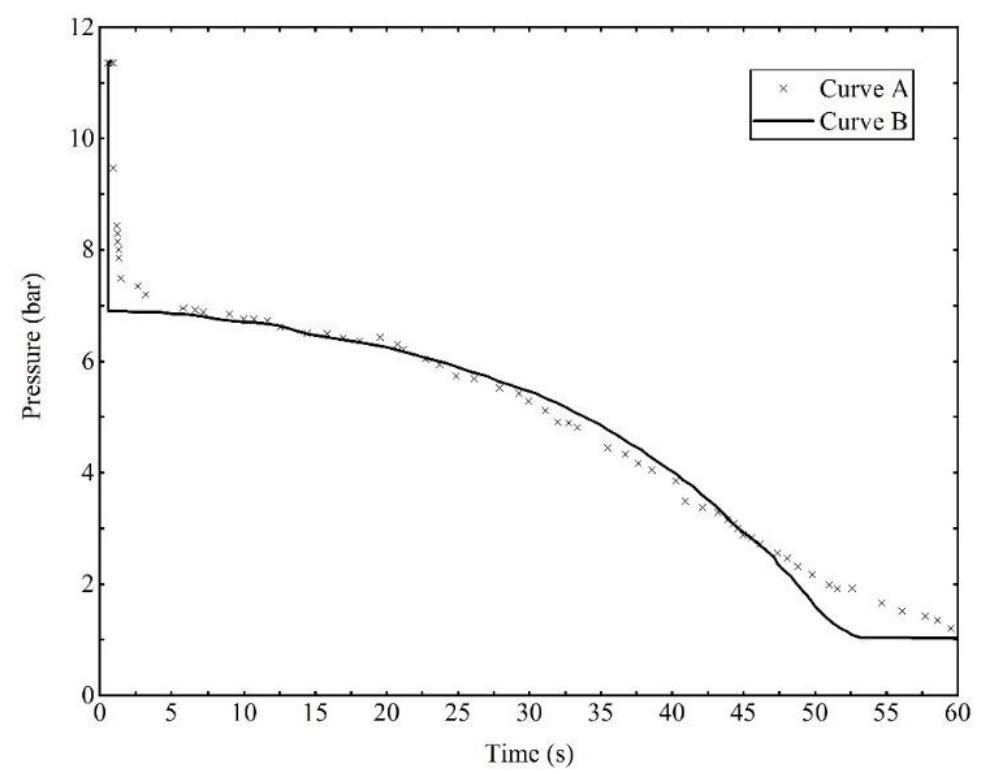

Figure 2.17: Pressure-time profiles at open end for the Isle of Grain P45 test (LPG). Curve A: field data; Curve B: model predictions (Mahgerefteh et al., 2011). 


\section{Piper Alpha Full Bore Rupture}

The HEM model was also validated against the closed end pressure data recorded following FBR of the subsea pipeline connecting Piper Alpha to MCP-01 platforms during the Piper Alpha disaster (Chen, 1993; Cullen, 1993). The fluid composition and pipeline initial conditions prior to FBR are respectively given in tables 2.2 and 2.3.

Table 2.2: Fluid compositions in subsea pipeline from Piper-Alpha to MCP-01 platforms (Chen, 1993).

\begin{tabular}{|ll|}
\hline Component & Mole $(\%)$ \\
\hline $\mathrm{CH}_{4}$ & 73.6 \\
$\mathrm{C}_{2} \mathrm{H}_{6}$ & 13.4 \\
$\mathrm{C}_{3} \mathrm{H}_{8}$ & 7.4 \\
i- $\mathrm{C}_{4} \mathrm{H}_{10}$ & 0.4 \\
n- $\mathrm{C}_{4} \mathrm{H}_{10}$ & 1.0 \\
i- $\mathrm{C}_{5} \mathrm{H}_{12}$ & 0.08 \\
n- $\mathrm{C}_{5} \mathrm{H}_{12}$ & 0.07 \\
$n-\mathrm{C}_{6} \mathrm{H}_{14}$ & 0.02 \\
$\mathrm{~N}_{2}$ & 4.03 \\
\hline
\end{tabular}

Table 2.3: Initial conditions prior to FBR of the subsea pipeline between Piper Alpha to MCP01 platforms (Chen, 1993).

\begin{tabular}{|ll|}
\hline Initial Condition & Value \\
\hline Pipeline Length (km) & 54 \\
Inner Diameter (mm) & 419.1 \\
Pipeline Thickness (mm) & 19 \\
Pipeline Roughness (mm) & 0.26 \\
Initial Pressure (bar) & 117 \\
Initial Temperature (K) & 283 \\
Ambient Temperature (K) & 283 \\
\hline
\end{tabular}


Figure 2.18 shows the comparison between field data and model predictions for pressure-time profiles at open end of the subsea pipeline between Piper Alpha to MCP-01 platforms.

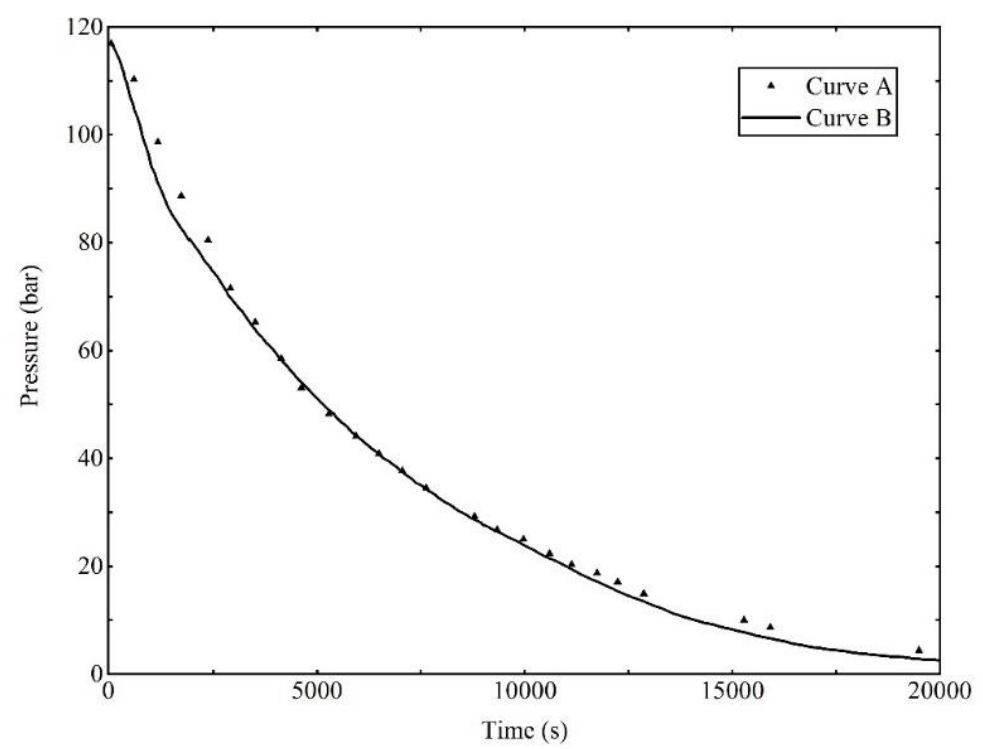

Figure 2.18: Pressure-time profiles at open end of the subsea pipeline between Piper Alpha to MCP-01 platforms. Curve A: field data; Curve B: model predictions (Mahgerefteh et al., 1999).

As it can be observed from the figure, the predicted data is in very good agreement with field data. The largest deflection of ca. $3 \%$ is shown at around $1500 \mathrm{~s}$, which corresponds to the onset of gas/liquid transition at the closed end of the pipeline.

\subsubsection{The Simulation of the Dynamic Response of Emergency Shutdown Valves Following Pipeline Failure}

Mahgerefteh et al. (1997) introduced a set of new boundary conditions for simulating the dynamic response of ESDVs including non-return Check Valves (CVs) and ball-type Remotely Controlled Valves (RCVs). The inventory was treated as an ideal gas in order to illustrate the various valve closure dynamic effects in gas transmission pipelines. The details of the boundary conditions were given in Section 2.5.5.

As part of the model verification, a numerical test was conducted for valve response following the emergency isolation of a methane pipeline of $145 \mathrm{~km}$ length, $0.87 \mathrm{~m}$ i.d. during its FBR 
decompression. The initial flow velocity was $10 \mathrm{~m} / \mathrm{s}$ and the line pressure and temperature were 133 bar and $283 \mathrm{~K}$ respectively. FBR was assumed to be initiated at one end of the test pipe, and the RCV was assumed to activate at a 10 bar drop below the normal pipeline operating pressure following the release, closing at a rate of $2.54 \mathrm{~cm} / \mathrm{s}$.

Mahgerefteh et al. (1997) investigated the effect of valve proximity to the rupture plane on the total amount of inventory released. Figure 2.19 shows the variation of inventory loss with distance of valve from the rupture plane in the case of a RCV and a CV. The latter was assumed to close upon flow reversal.

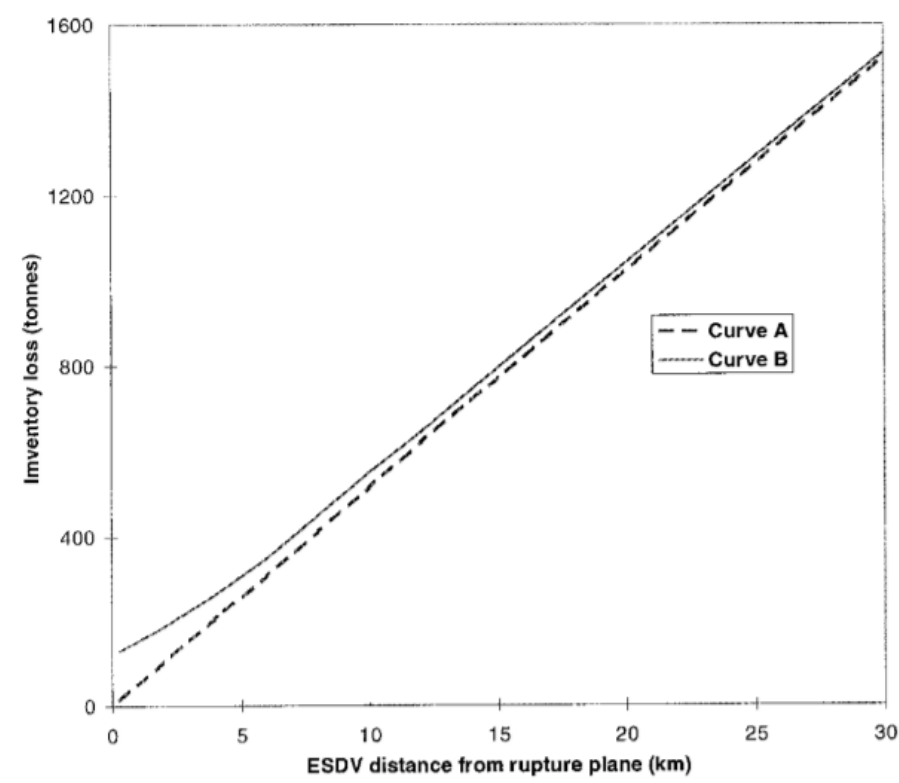

Figure 2.19: The variation of inventory loss as a function of ESDV proximity to the rupture plane: curve A; CV; curve B; RCV (Mahgerefteh et al., 1997).

It is clear from figure 2.19 that for valves positioned in close proximity (up to $5 \mathrm{~km}$ ) to the rupture plane, a CV offers a much better degree of protection in terms of limiting the total amount of inventory released. However, for valves positioned at larger distances, the difference in their performance becomes far less discernible.

Figure 2.20 presents the corresponding variation of the $\%$ underestimate of inventory released as a function of ESDV distance to the rupture plane. 


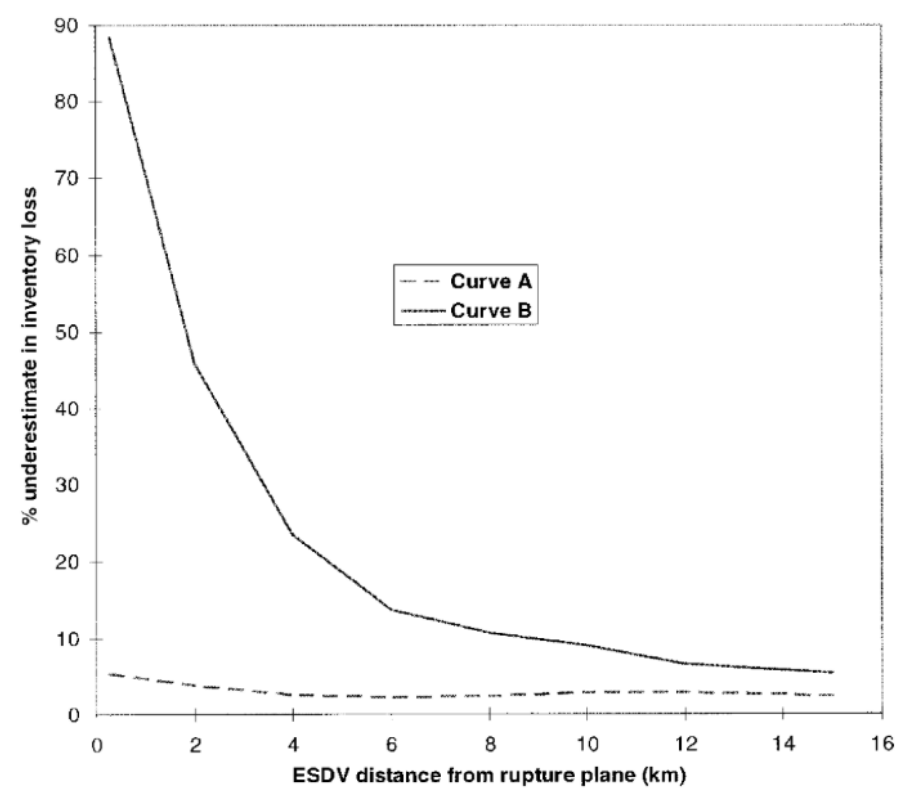

Figure 2.20: The variation of inventory loss as a function of ESDV proximity to the rupture plane: curve A; CV; curve B; RCV (Mahgerefteh et al., 1997).

It is interesting to note from figure 2.20 that a significant underestimate in the mass of fluid escaping can be observed based on the assumption that the total inventory released following ESDV closure is equal to that within the isolable section of the pipeline prior to FBR. This is especially the case as the RCV is placed closer to the rupture plane.

\subsubsection{Terenzi (2005)}

Terenzi (2005) developed a simulation tool, Machnet (referred as Machnet_Real), using the HEM assumption to investigate the impact of real gas behaviour on the interaction between pipeline decompression and ductile fracture propagation. Both PR (Peng and Robinson, 1976) and Soave-Redlich-Kwong (SRK) (Soave, 1972) EoSs were used to determine the fluid thermophysical properties and phase equilibrium data. Fluid/wall heat transfer was calculated by solving the Fourier equation in cylindrical geometry between the external environment and the fluid. The Colebrook-White correlation (Keenan and Neumann, 1946) was used to account for the frictional effects along the pipeline. The resulting governing system of equations was resolved using Roe's (Hirsch, 2007) approximate Riemann solver in an explicit Finite Volume Godunov-type scheme. 
Terenzi (2005) also developed a model for the decompression of a pipeline transporting a perfect gas (referred to as Machnet_Ideal) by assuming zero heat transfer and frictionless flow to determine the pressure at the exit plane and the speed of the rarefaction wave.

Machnet_Ideal and Machnet_Real's predictions were validated by comparison with the results of Test no.5 conducted at the Foothills Pipelines Northern Alberta Burst Test Facility (NABT) (Foothills Pipe Lines (Yukon) Ltd, 1981; Picard and Bishnoi, 1988), where the pipeline was ruptured at its midpoint and decompression data were obtained for each half. This test involved the release of natural gas (ca. $85 \%$ methane) from a $60 \mathrm{~m}$ long pipeline with pipeline internal diameter, pressure and temperature of $1422 \mathrm{~mm}, 7.54 \mathrm{MPa}$ and $291.65 \mathrm{~K}$, respectively. The fluid composition is given in table 2.4 .

Table 2.4: Fluid composition of NABT Test no. 5 (Picard and Bishnoi, 1988).

\begin{tabular}{|ll|}
\hline Component & Mole (\%) \\
\hline $\mathrm{CH}_{4}$ & 84.694 \\
$\mathrm{C}_{2} \mathrm{H}_{6}$ & 8.212 \\
$\mathrm{C}_{3} \mathrm{H}_{8}$ & 4.379 \\
i- $\mathrm{C}_{4} \mathrm{H}_{10}$ & 0.201 \\
n- $\mathrm{C}_{4} \mathrm{H}_{10}$ & 0.235 \\
i- $\mathrm{C}_{5} \mathrm{H}_{12}$ & 00.029 \\
n- $\mathrm{C}_{5} \mathrm{H}_{12}$ & 0.030 \\
n- $\mathrm{C}_{6} \mathrm{H}_{14}$ & 0.008 \\
$\mathrm{~N}_{2}$ & 2.212 \\
\hline
\end{tabular}

In figure 2.21, the variation of the simulated ratio of pressure to initial pressure and void fraction as a function of expansion wave velocity is presented. The void fraction profile in the expansion wave is shown along the pressure ratio; in this case gas only is presented in the pipeline initial condition before rupture, and some liquid is formed during the expansion wave propagation. 


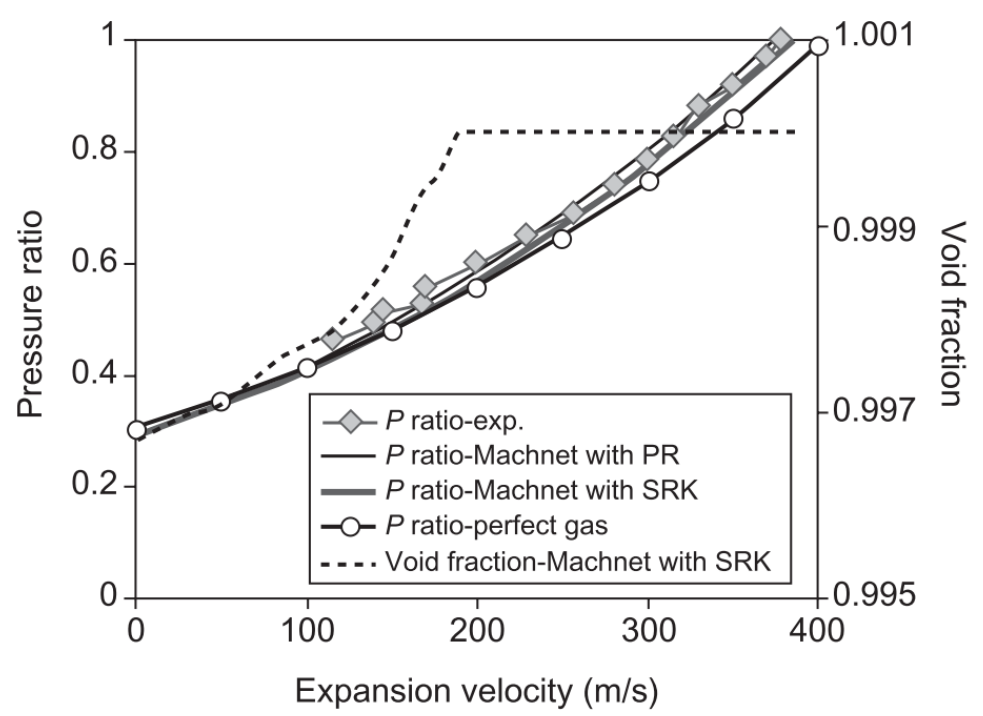

Figure 2.21: Measured and calculated expansion velocity results as a function of pressure ratio and void fraction of NABT Test 5 (Terenzi, 2005). The solid line with diamond box presents the experimental data, while other lines present simulated results.

As it may be observed, Machnet_Real coupled with either the PR or SRK EoS shows similar behaviour, while Machnet_Ideal shows a difference in the expansion wave pressure prediction for a selected value of expansion velocity. For instance, at an expansion velocity of $350 \mathrm{~m} / \mathrm{s}$, the real fluid model predicts a pressure ratio ca. 0.85 , and the perfect gas predicts ca. 0.75 pressure ratio. In addition, at the point when the pressure ratio reaches ca. 0.55, Machnet_Real predictions using the PR and SRK EoS begin to diverge from the experimental data. Based on the above data it is clear that the fluid flow model is capable of simulating the decompression wave initiated upon pipeline failure. However, the authors did not present a similar comparison relating to the release characteristics (e.g. the release pressure, temperature and discharge mass flowrate as a function of time). As such, the efficacy of the model with regards to the prediction of outflow cannot be verified.

\subsubsection{Lund at el. (2011)}

Lund at el. (2011) presented a HEM based model for investigating the depressurisation of twophase $\mathrm{CO}_{2}$ in high-pressure pipelines. The stiffened-gas EoS (Menikoff and Plohr, 1989; Menikoff, 2007) was used for calculating fluid thermophysical properties and phase equilibrium data. The fluid/wall heat transfer calculation and the friction factor used are not 
disclosed by the authors. The study compared the convergence and accuracy of two numerical methods, namely the multi-stage centred (MUSTA) scheme (Toro, 2003; Titarev and Toro, 2005) and the upwind Roe scheme (Roe, 1981).

The flow model was applied to a hypothetical $100 \mathrm{~m}$ long pure $\mathrm{CO}_{2}$ pipeline. The pipeline diameter and wall thickness were not given. The initial pressure and temperature inside pipe were respectively at $60 \mathrm{bar}$ and $288 \mathrm{~K}$. The fluid was assumed to release from one end of the pipe following a FBR and exposed to an external pressure of 30 bar. The Courant-FredrichLewy (CFL) number used was 0.9. The number of grid cells was varied from 50 to 6400. It is noted that only the right-most part of the domain (i.e. 85 to $100 \mathrm{~m}$ ) was shown for all tests results, given that these produced the best convergence

Figures 2.22 and 2.23 respectively show convergence test results for the Roe method with Superbee limiter (Roe SB) (Roe, 1985) and the MUSTA method. Figure 2.24 shows the results for two methods compared.

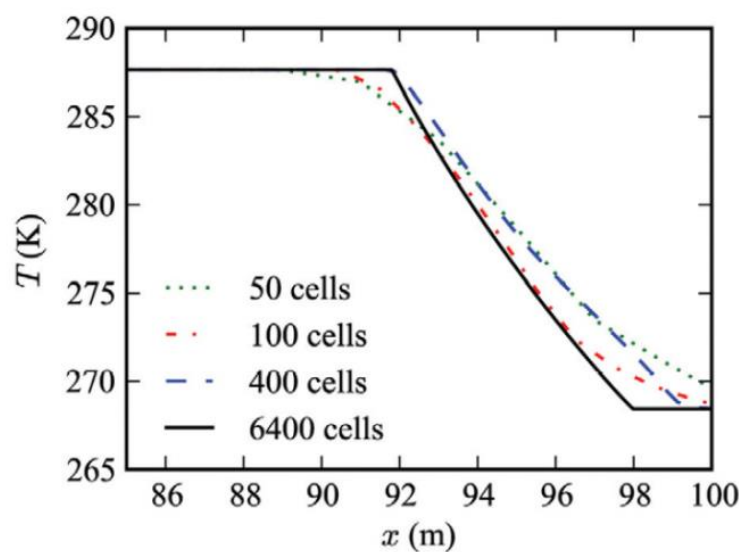

(a) Temperature

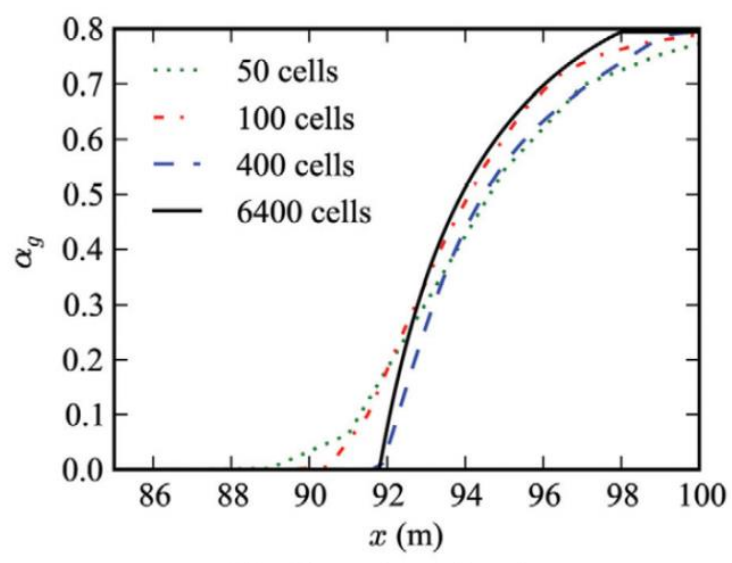

(b) Gas volume fraction

Figure 2.22: Convergence test for the Roe method with Superbee limiter (Roe SB). Note that only the right-most part of the domain is shown (Lund et al., 2011). 


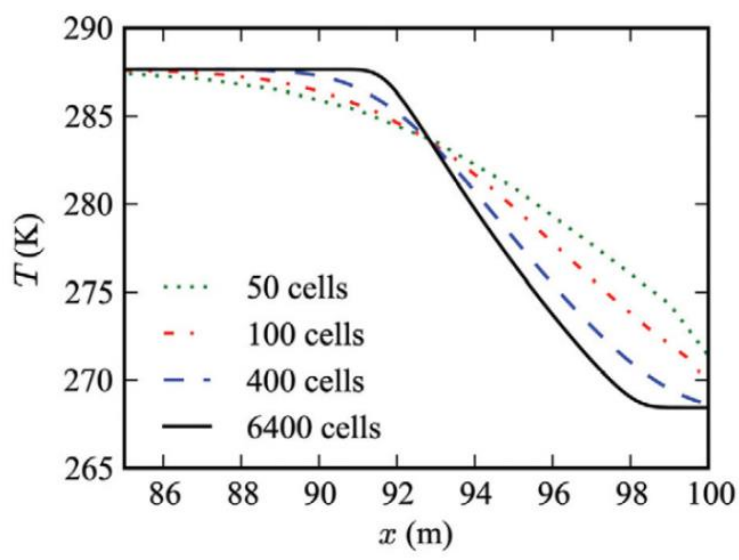

(a) Temperature

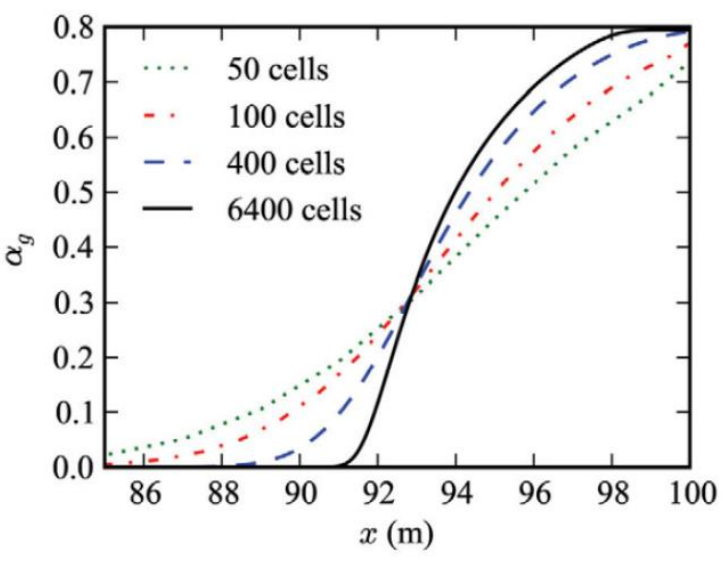

(b) Gas volume fraction

Figure 2.23: Convergence test for the MUSTA method. Note that only the right-most part of the domain is shown (Lund et al., 2011).

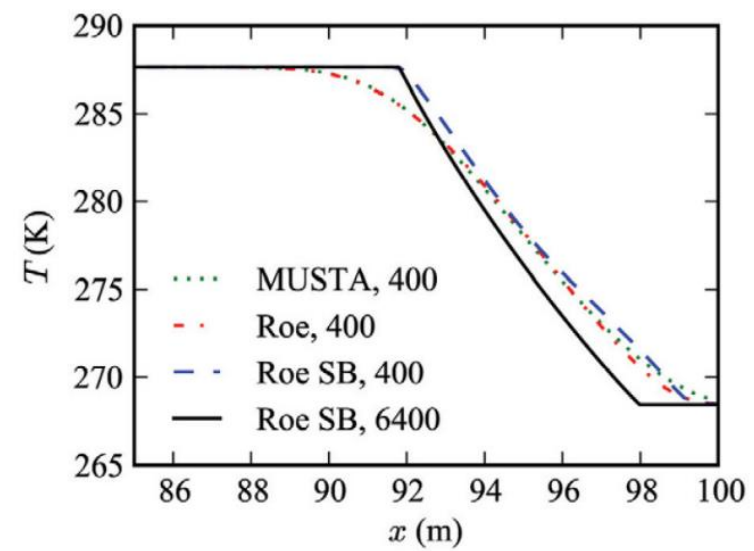

(a) Temperature

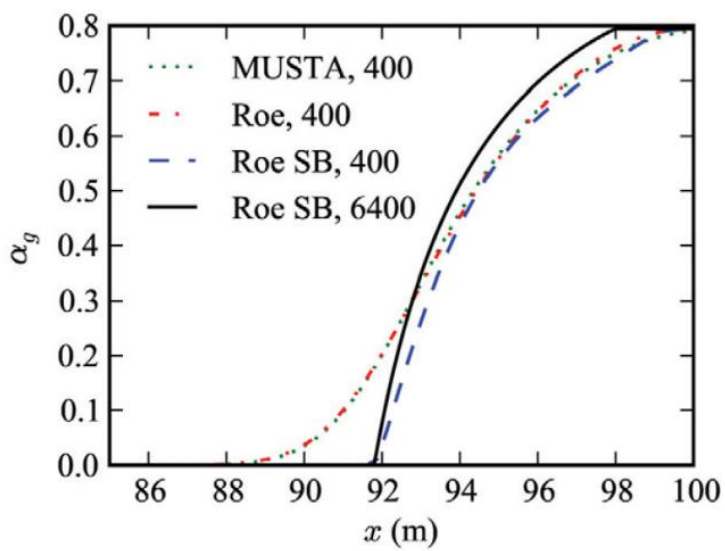

(b) Gas volume fraction

Figure 2.24: Comparison of convergence for the different methods, only the right-most part of the domain is shown (Roe, 1985).

From figures 2.22 and 2.23, it can be observed that for the Roe method with Superbee limiter (Roe SB), the solution converges nicely. The MUSTA method also converges, but slightly less sharp corners can be observed. According to the authors, this observation was expected as centred methods are more diffusive than upwind methods. Focusing on figure 2.24, it can be seen that the MUSTA method and the Roe method produce comparable results. The higherorder Roe SB is far closer to the converged solution. The authors concluded that these results illustrated a higher-order method like Roe SB significantly improves the resolution of a 
depressurisation wave. Unfortunately, no validation against appropriate experimental data was provided by the authors to support their model predictions.

\subsubsection{Teng et al. (2016)}

Teng et al. (2016) investigated the decompression characteristics of a $\mathrm{CO}_{2}$ pipeline using experimental as well as mathematical approaches. The experimental tests were performed based on a $70 \mathrm{~m}, 25 \mathrm{~mm}$ i.d. stainless steel (type 304) pipeline. An electric heating jacket was installed to control the temperature of $\mathrm{CO}_{2}$ inside the pipeline. Pressure and temperature sensors were used to monitor the variations of pressure and temperature as a function of time. Three tests were conducted simulating the $\mathrm{CO}_{2}$ pipeline decompression following a FBR at the end of the pipeline. The experimental conditions are shown in table 2.5.

Table 2.5: Experimental conditions (Teng et al., 2016).

\begin{tabular}{|cccc|}
\hline Test No. & Initial Pressure (bar) & Initial Temperature $\left({ }^{\circ} \mathbf{C}\right)$ & Phase state \\
\hline 1 & 75 & 40 & Supercritical \\
2 & 55 & 5 & Liquid \\
3 & 35 & 15 & Vapour \\
\hline
\end{tabular}

The flow model was based on the HEM assumption and PR EoS was applied for predicting the fluid thermophysical properties and equilibrium data. The fluid/wall heat transfer calculation and the friction factor used were not disclosed by the authors. Same as the model developed by Mahgerefteh et al. (1999), the MOC was implemented to solve the conservation equations. The model predictions were compared with the experimental data.

Figures 2.25 to 2.27 show the comparison of predicted and measured pressure variation as a function of time at both inlet and outlet of the test pipeline in supercritical, liquid and vapour phases respectively. 


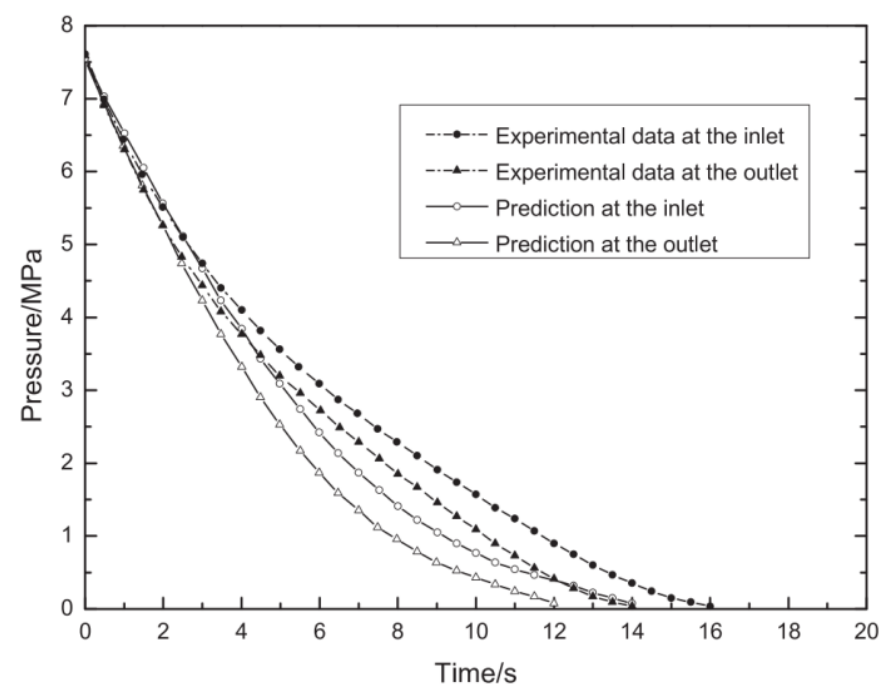

Figure 2.25: Predicted and measured pressure variation as a function of time at both inlet and outlet of the test pipeline in supercritical phase (Teng et al., 2016).

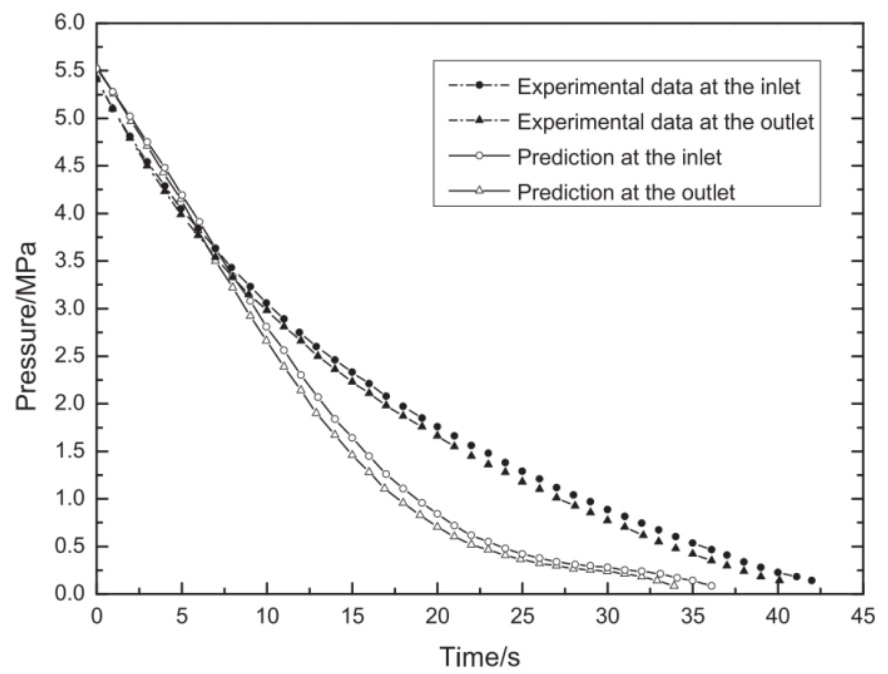

Figure 2.26: Predicted and measured pressure variation as a function of time at both inlet and outlet of the test pipeline in liquid phase (Teng et al., 2016). 


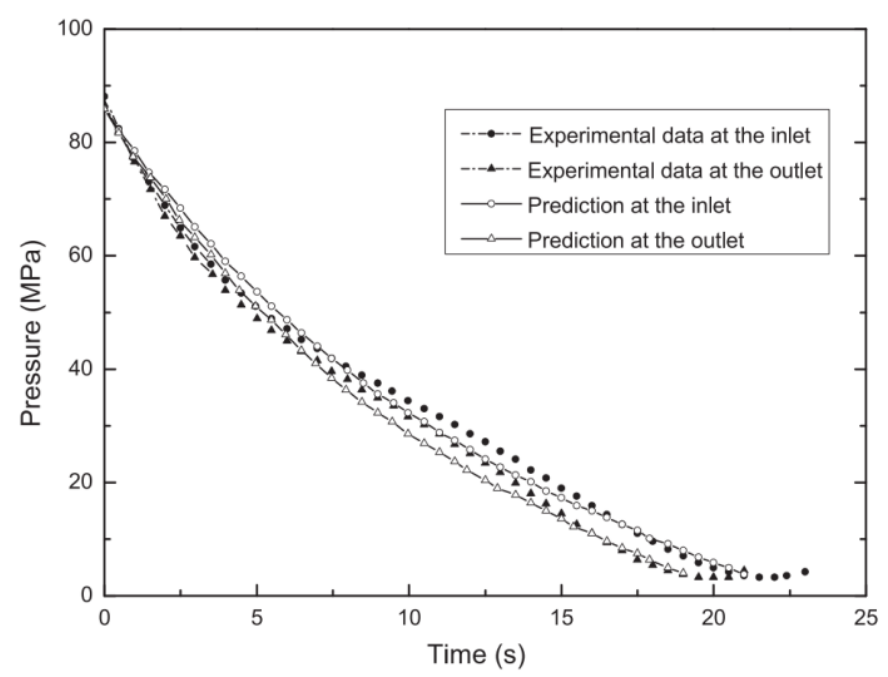

Figure 2.27: Predicted and measured pressure variation as a function of time at both inlet and outlet of the test pipeline in vapour phase (Teng et al., 2016).

As can be seen from figures 2.25 to 2.27 , there is good agreement between the model predictions and the measured data for all tests during the early stage of the release. Following this, the model shows reasonably accurate predictions starting from vapour phase $\mathrm{CO}_{2}$ (figure 2.27). However, in the tests for initially supercritical and liquid phases $\mathrm{CO}_{2}$ (figures 2.25 and 2.26 respectively), significant over-predictions of rate of decompression in pressure can be observed. According to the authors, such observation for liquid phase $\mathrm{CO}_{2}$ test was due to the earlier estimation of the onset of phase transition based on the model as compared to that from the test. No explanation was provided by the authors regarding the above for the test starting from supercritical phase $\mathrm{CO}_{2}$.

\subsubsection{Xu et al. (2014)}

Xu et al. (2014) presented the development and validation of a pipeline depressurisation model based on the HEM assumption. The friction factor given by Massey (1998), was applied to determine the fluid/wall drag interaction. Shah's correlation (Shah, 1979) and Gungor's correlation (Gungor and Winterton, 1986) were respectively used to calculate the heat transfer coefficient for condensation heat transfer and boiling heat transfer. In the case of single-phase flow, the PR-EoS was implemented to calculate fluid thermophysical properties and phase 
equilibrium data. For the two-phase flow, the method developed by Mehra et al. (1983) was used to calculate liquid-vapor phase equilibrium. A finite volume approach based on the arbitrary Lagrangian -Eulerian (ALE) (Hirt et al., 1997) was employed to solve the conservation equations.

The model was validated against the Botros's experiment (Botros et al., 2007) by comparing predicted and measured pressure and temperature as a function of time. The experiment was performed based on a $172 \mathrm{~m}, 49 \mathrm{~mm}$ i.d. stainless steel rich gas pipeline with a FBR occurred at the end of the test pipeline. The wall thickness and pipeline roughness were $5 \mathrm{~mm}$ and 0.05 $\mathrm{mm}$, respectively. The initial temperature and pressure were at $-4.99{ }^{\circ} \mathrm{C}$ and $99.49 \mathrm{bar}$ respectively. The fluid composition is given in table 2.6. Several sets of high-frequency pressure and temperature transducers were installed along the pipeline to monitor the pressure and temperature following rupture. The locations of the monitoring points are given in table 2.7 .

Table 2.6: Fluid composition of Botros's experiment (Xu et al., 2014).

\begin{tabular}{|ll|}
\hline Component & Mole $(\%)$ \\
\hline $\mathrm{CH}_{4}$ & 68.509 \\
$\mathrm{C}_{2} \mathrm{H}_{6}$ & 21.406 \\
$\mathrm{C}_{3} \mathrm{H}_{8}$ & 9.08 \\
$\mathrm{i}-\mathrm{C}_{4} \mathrm{H}_{10}$ & 0.026 \\
$n-\mathrm{C}_{4} \mathrm{H}_{10}$ & 0.014 \\
$\mathrm{i}-\mathrm{C}_{5} \mathrm{H}_{12}$ & 0.002 \\
$n-\mathrm{C}_{5} \mathrm{H}_{12}$ & 0.002 \\
$n-\mathrm{C}_{6} \mathrm{H}_{14}$ & 0.001 \\
$\mathrm{CO}_{2}$ & 0.553 \\
$\mathrm{~N}_{2}$ & 0.408 \\
\hline
\end{tabular}

Table 2.7: Monitoring locations of the Botros's experiment (Xu et al., 2014).

\begin{tabular}{|ll|}
\hline Monitoring Points & Locations from Rupture (m) \\
\hline P8, T8 & 1.64 \\
\hline
\end{tabular}




\begin{tabular}{|ll|}
\hline P10 & 4.04 \\
P11 & 8.04 \\
P14, T14 & 23.056 \\
P19, T19 & 47.072 \\
P24, T24 & 71.088 \\
P31, T31 & 103.157 \\
P36, T36 & 127.173 \\
\hline
\end{tabular}

Figure 2.28 shows the comparison of the model predictions and the measured data for the Botros's experiment for the pressure variation as a function of time at different monitoring locations.
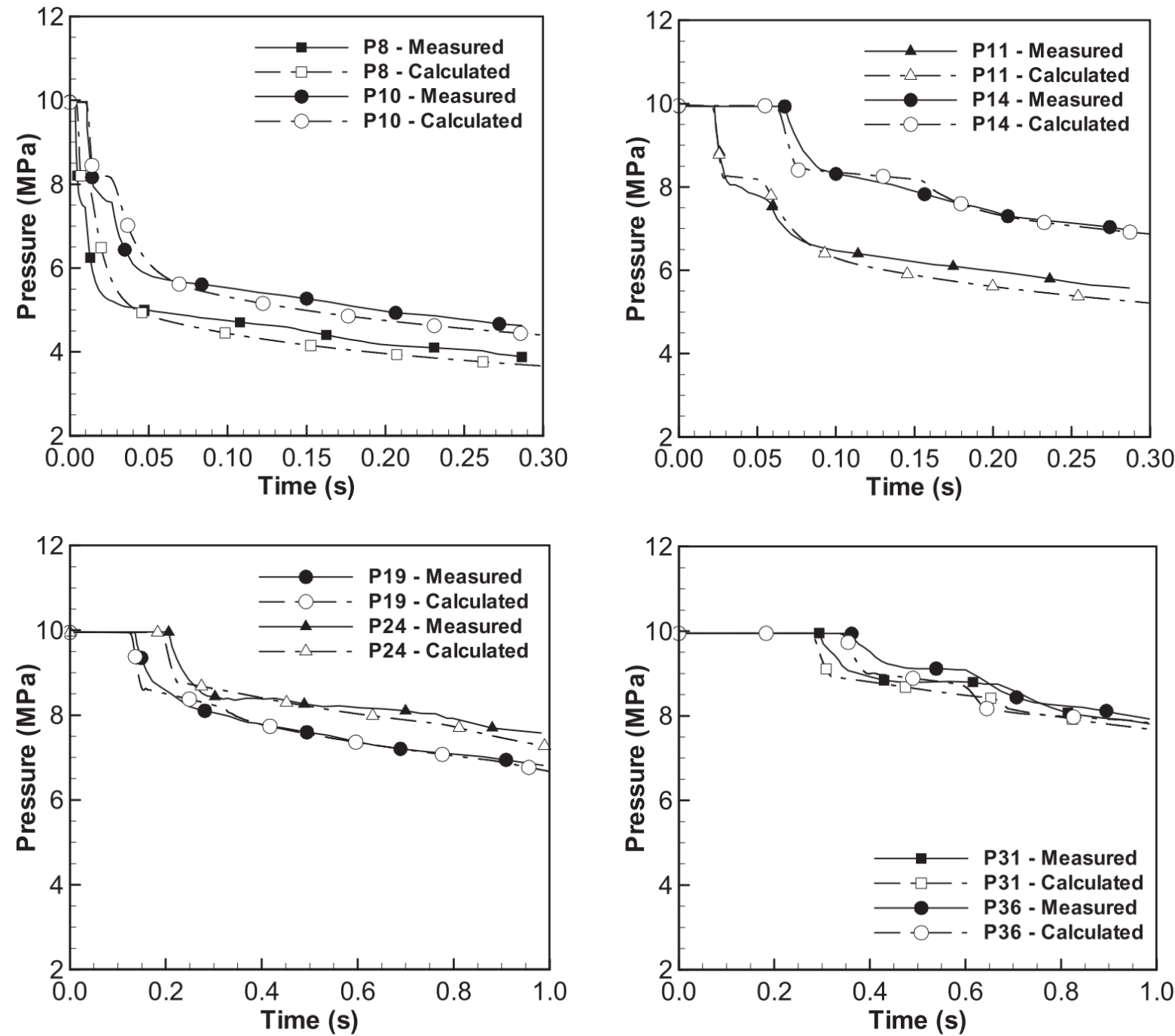

Figure 2.28: Model predictions and the measured data for pressure variations with time at different monitoring locations for the Botros's experiment (Xu et al., 2014). 
In figure 2.28, sharp drop in pressure can be observed following the rupture for the monitoring locations near the rupture planes (P8 and P10) due to the extremely fast decompression rate. The decompression rate becomes slower for the monitoring locations further away from the rupture plane. It is noteworthy that an apparent recovery in pressure can be seen around ca. 80 bar for predicted data in these monitoring locations. The authors pointed out that this was due to the phase transition when the pipeline pressure dropped to around the saturation pressure.

Figure 2.29 presents the comparison of model predictions and the measured data for the Botros's experiment for the variation temperature as a function of time at different monitoring locations.
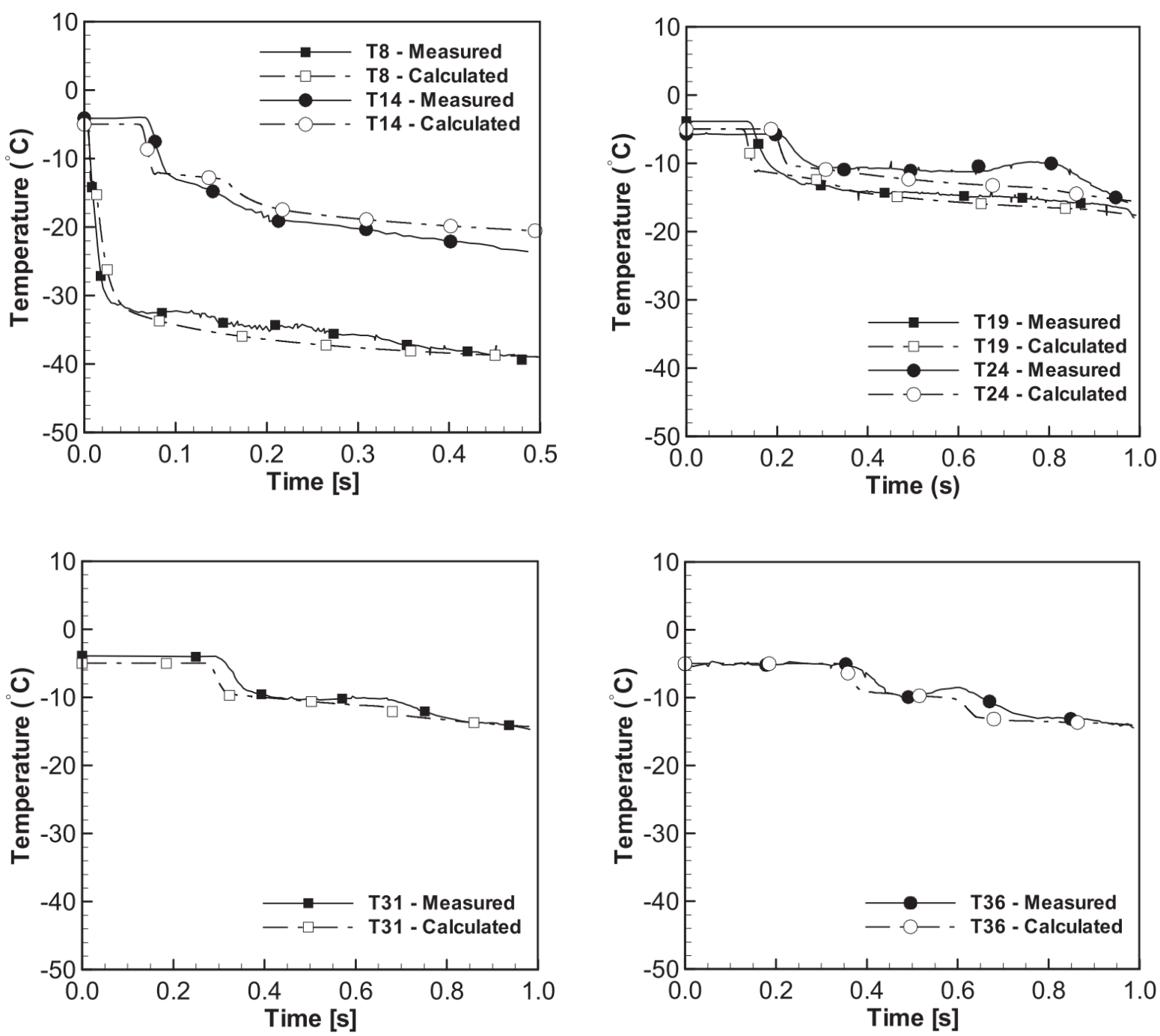

Figure 2.29: Model predictions and the measured data for temperature variations with time at different monitoring locations for the Botros's experiment (Xu et al., 2014). 
From figure 2.29, it may be observed that the temperature profiles follow similar trends to the corresponding pressure profiles given in figure 2.28. In all monitoring locations, good agreement between predicted and field data was obtained for the Botros's experiment.

\subsubsection{Summary of the HEM model applications}

Table 2.8 presents the summary of the reviewed HEM applications highlighting their merits/ disadvantages in simulating pipeline outflow during decompression. 
Table 2.8: The summary of the reviewed HEM applications.

\begin{tabular}{|c|c|c|}
\hline HEM applications & Merits & Disadvantages \\
\hline $\begin{array}{l}\text { Mahgerefteh et al. (1997, } \\
\text { 1999, 2000, 2007, 2008) }\end{array}$ & $\begin{array}{l}\text { - the most validated among its kind reported in open } \\
\text { literature for both hydrocarbons and } \mathrm{CO}_{2} \text { large- } \\
\text { scale high-pressure pipelines } \\
\text { - good agreement between model predictions and } \\
\text { experimental data } \\
\text { - successfully extended the model to account for } \\
\text { other important features, including the dynamic } \\
\text { response of ESDVs, the development of a } \\
\text { simplified analytically based vessel blowdown } \\
\text { model, decompression of pipeline networks and } \\
\text { ductile fracture propagation of the pipe wall }\end{array}$ & $\begin{array}{l}\text { - essential questions for the ESDV study such as the } \\
\text { resulting pressure surges upon CV closure, the } \\
\text { impact of ASV and RCV activation pressure in } \\
\text { isolating the outflow flow following pipeline } \\
\text { failure, and the efficacy of using different } \\
\text { combinations of inline ESDV have not been } \\
\text { addressed }\end{array}$ \\
\hline Terenzi (2005) & $\begin{array}{l}\text { - good agreement between the expansion wave } \\
\text { pressure predictions and the measured data } \\
\text { - capable of simulating the decompression wave } \\
\text { initiated upon pipeline failure }\end{array}$ & $\begin{array}{l}\text { - no comparison relating to the release characteristics } \\
\text { was presented, the efficacy of the model with } \\
\text { regards to the prediction of outflow cannot be } \\
\text { verified }\end{array}$ \\
\hline
\end{tabular}




\begin{tabular}{|c|c|c|}
\hline Lund at el. (2011) & $\begin{array}{l}\text { - the Roe method with Superbee limiter converged } \\
\text { nicely } \\
\text { - the MUSTA method and the Roe method produced } \\
\text { comparable results } \\
\text { - higher-order method significantly improved the } \\
\text { resolution of a depressurisation wave }\end{array}$ & $\begin{array}{l}\text { - no validation against appropriate experimental data } \\
\text { was provided to support the model predictions }\end{array}$ \\
\hline Teng et al. (2016) & $\begin{array}{l}\text { - good agreement between the predicted and } \\
\text { measured pressure variation for all tests during the } \\
\text { early stage of the release } \\
\text { - reasonably accurate predictions for the test starting } \\
\text { from vapour phase } \mathrm{CO}_{2} \text { at later stage of the release }\end{array}$ & $\begin{array}{l}\text { - no explanation was provided regarding the over- } \\
\text { predictions of rate of decompression in pressure for } \\
\text { the test starting from supercritical phase } \mathrm{CO}_{2}\end{array}$ \\
\hline Xu et al. (2014) & $\begin{array}{l}\text { - good agreement between predicted and field data } \\
\text { for the Botros's experiment }\end{array}$ & $\begin{array}{l}\text { - the authors claimed that the model improved the } \\
\text { computational efficiency, however, no details on } \\
\text { the computational runtimes were given }\end{array}$ \\
\hline
\end{tabular}




\subsection{Concluding Remarks}

In this chapter, the mass, momentum and energy conservation equations for simulating transient fluid flow in a high-pressure pipeline following its accidental failure were presented. These equations were expressed with pressure, enthalpy and velocity as the primitive variables as opposed to pressure, entropy and velocity given their superior accuracy and computational efficiency. The Homogeneous Equilibrium Mixture assumption was applied given the good agreement obtained based on comparison of the model predictions against the available field and experimental data.

The various hydrodynamic and thermodynamic relations for predicting the pertinent fluid properties such as the fluid speed of sound, two-phase mixture density, fluid/wall friction and heat transfer were presented. The PR EoS was coupled with the conservation equations for predicting the pertinent fluid thermophysical properties and phase equilibrium data required for simulating the transient fluid flow.

Given the likelihood of pipeline failure occurring during normal operation (i.e. involving feed flow prior to failure), the development of the steady state isothermal flow model for predicting the required fluid properties along the pipeline as the initial conditions for simulating post pipeline failure was presented.

The formulation of the MOC based on the Method of Specified Time Intervals to solve the governing conservation equations using compatibility equations which were in turn discretised using the Euler predictor corrector technique was presented. Various boundary conditions were introduced in combination with the compatibility equations to model the fluid as well as ESDV closure dynamics following pipeline failure.

The HEM model presented in this chapter is novel in the following aspects including:

- the development of an open pipe end downstream boundary condition

- the modifications of choked flow calculation in the case of a pipeline FBR failure

- the extension of ESDV boundary conditions developed by Mahgerefteh et al. (1997) to account for inline CVs, RCVs and ASVs closure dynamics response 
Finally, a review focusing on the applications of the HEM model, and its various extensions for predicting pipeline decompression was presented. Based on the review, the HEM model is shown to perform reasonably well as compared to measured data for pipeline decompression tests. Moreover, the HEM model has been successfully adopted in investigating the dynamic response of ESDVs by applying the appropriate boundary conditions.

\section{Gaps in knowledge:}

Considering the state of the art, significant gaps in applying the HEM model for investigating the impact of ESDVs on minimising outflow following pipeline failures exists.

For example, previous studies on ESDVs only focus on investigating the dynamic response of valve closure following a pipeline failure such as the effects of RCV and CV proximity to the rupture plane and the delay in their closure on the total amount of inventory released prior to pipeline isolation. However, essential questions such as the resulting pressure surges upon CV closure, the impact of ASV and RCV activation pressure in isolating the outflow flow following pipeline failure, and importantly, the efficacy of using different combinations of inline ESDV have not been addressed.

Furthermore, an important consideration in practice is the significant capital cost of ESDVs, which can become prohibitive depending on their type (ASVs or RCVs are much more expensive than CVs) and number of units employed. As such, appropriate inline ESDV configurations encompassing valve type, combination, number, strategic positioning and operational settings must be carefully selected to minimise costs while still providing adequate protection in the event of a pipeline failure. Such challenge can be set as a multi-objective problem to be resolved accordingly. At the same time, bearing in mind the myriad of design and control parameters involved, the optimisation procedure must also be computationally efficient and easy to visualise.

Considering the above, Chapter 3 presents a review of the development of multi-objective optimisation methods, including the general approaches for selecting the optimal solutions, the specific techniques to visualise the possible solutions along with a survey of the relevant applications. Given scarcity of the application of such methods to inline ESDV pipeline configurations, relevant work on the application of multi-objective optimisation techniques to pipeline network design and operation is also reviewed. 


\section{Chapter 3:}

\section{Multi-objective Optimisation and Its Applications}

\subsection{Introduction}

Mathematically, optimisation can be explained as the selection of a best element under certain criterions from a set of available possible choices (Wright, 2016). Optimisation is widely used in various areas in all quantitative disciplines including engineering, computer science and operations research, and many ways of naming and classifying optimisation problems can be found in open literature (Kirkpatrick et al., 1983; Du et al., 2008; Yang, 2010). According to Yang (2010), a common classification can be defined with regards to the number of objectives, number of constraints, landscape of the objective functions, function forms, type of design variables, computational response time and determinacy as presented in figure 3.1.

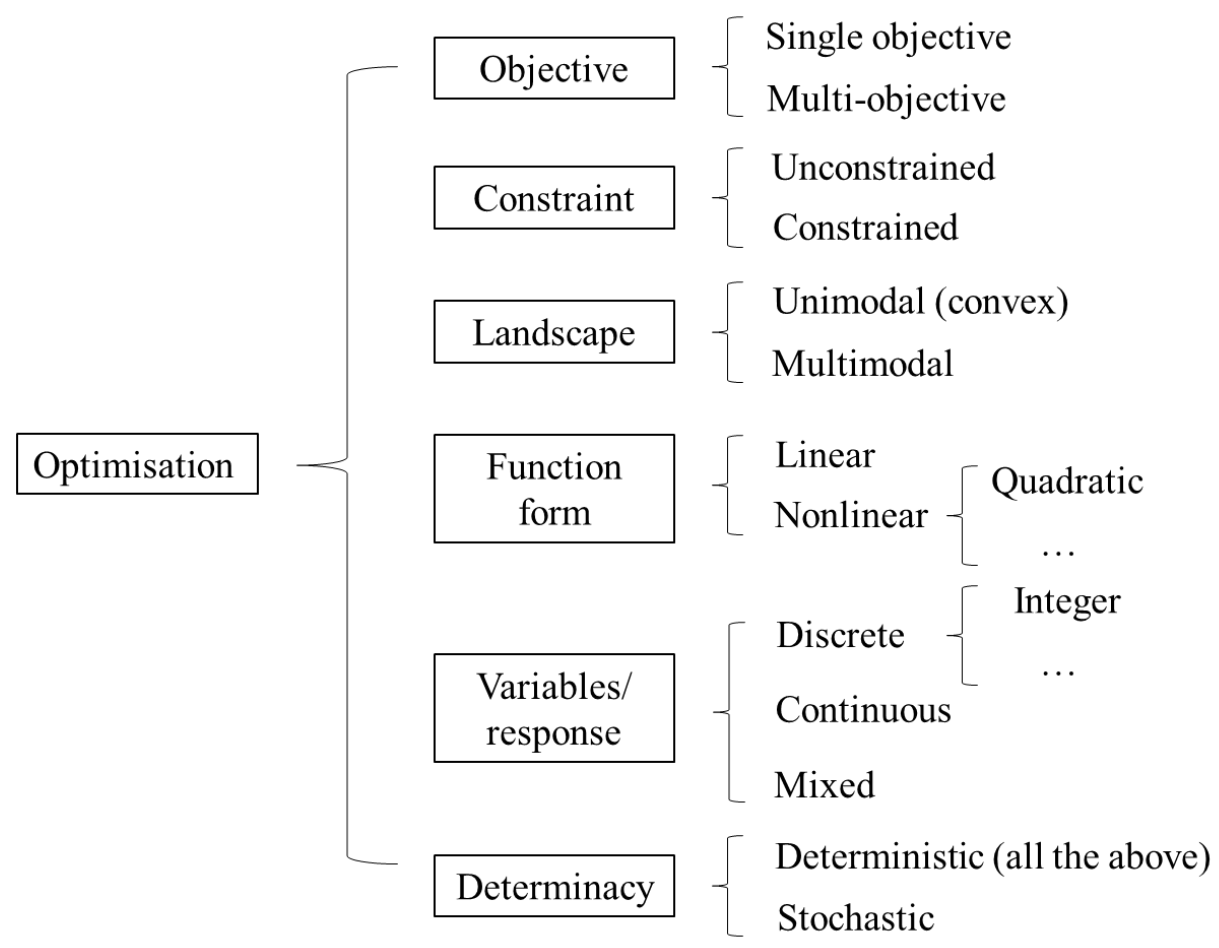

Figure 3.1: Classification of optimisation problems (Yang, 2010). 
As can be seen in figure 3.1, the optimisation problems can be classified into two categories based on the number of objectives: single objective and multi-objective. As discussed in Chapter 1, considering the significant capital cost of Emergency Shutdown Valves (ESDVs), the optimum configuration of ESDVs requires a delicate balance between their total cost, the total failure rate and the level of protection these provide in minimising the resulting risks in the event of a pipeline failure. According to figure 3.1, such challenge can be defined as a multi-objective problem.

Most optimisation problems in practice are multi-objective, and multi-objective optimisation methods are often used in many reliability-based and risk-informed activities of system design, operation, maintenance and regulation (Zio and Bazzo, 2011; Brown, et al., 2014; Demissie, 2015; HSE, 2017). The solution of a multi-objective optimisation problem is usually not unique, as there are several competing objective functions which constitute the problem. Hence, a set of possible solutions in the space of the decision variables can be identified where none is best for all objectives. This set is often referred to as Pareto Set (Akbari et al., 2014). The region in the space of the values of all objectives defined by all Pareto Set points is called Pareto Front (Keßler et al., 2016). To obtain the Pareto Set, or the discrete approximation of Pareto Front, the objective functions are normally sampled using different values of the independent optimisation variables. The Pareto Set or Pareto Front are always used as the solution of a multi-objective optimisation problem given the subjective preferences of a decision maker.

Given the above, this chapter presents a review of the development of multi-objective optimisation methods, including the general approaches for selecting the optimal solutions; the specific techniques to visualise the Pareto Front and a survey of the relevant applications. Unfortunately, very few studies can be found in literature in applying such methods for inline ESDV pipeline configurations. Therefore, relevant work on the application of multi-objective optimisation techniques to pipeline network design and operation is also reviewed. 


\subsection{General Approaches and Visualisation Techniques}

\subsubsection{General Approaches for Selecting the Optimal Solutions}

There are different approaches to select the optimal solutions for a multi-objective problem, a popular classification is based on the time when a decision maker interacts or provides additional preference information and can be categorised as follows: a priori methods, interactive methods and a posteriori methods (Bechikh et al., 2015).

In a priori methods, the decision maker needs to provide preference information before the optimisation solution process. Then, the preference information is transformed into the mathematical definition of the multi-objective problem itself in order to guides the search for optimal solutions towards a preferred region. Many examples can be found in open literature to achieve the above including weighting the objectives differently (Yang, 1996), changing the definition of dominance (Molina et al., 2009), selecting a utility function which explains the decision maker's behaviour and interest in the alternative solutions (Malakooti, 1988) and giving the objectives reference values and priority levels (Yang, 2000). However, in such methods, the decision maker does not necessarily know the limitations and possibilities of the problem which can results in inaccurate or even misleading outcomes (Corne and Knowles, 2007).

In contrast with a priori methods, a set of Pareto optimal solutions are obtained using an optimisation algorithm first in a posteriori methods. Thereafter, the decision maker can select the preferred solution among them. Typically, most existing evolutionary multi-objective optimisation (EMO) algorithms (Abraham and Jain, 2005) belong to this class. In a posteriori methods, the decision maker can have a better understanding of the trade-off relationships between the objectives. Nevertheless, the computational complexity may increase rapidly to obtain the Pareto Set as the number of objectives increases (Khare et al., 2003).

In interactive methods, the decision maker is able to participate and direct the optimisation process. As such, the solution pattern is formed and iteratively repeated based on the decision maker's preferences (Deb and Chaudhuri, 2005). The decision maker can always learn and modify his preferences during the process based on the domain knowledge acquired, for example stopping an iteration (e.g. trial-and-error) when reaching satisfactory results (Katagiri et al., 2008) and guiding the development of the process by removing alternatives depending 
on indicated preference strengths (Roy and Bouyssou, 1986). Compared with a priori or a posteriori methods, interactive methods require less computational cost as only those Pareto optimal solutions that the decision maker is interested in during the process are generated (Wang et al., 2017).

\subsubsection{Visualisation of Pareto Front}

In order to understand the trade-off solutions and thus determine a meaningful solution, specific techniques can be used to visualise the Pareto Front. A selection of visualisation techniques that are most commonly employed in practice are presented in the following.

- Scatter plot matrix. Scatter plot matrix is a straightforward visualisation technique to project all individual objective information of the underlying approximation set. This technique is the most frequently used in the case of two or three objectives. Although a scatter plot matrix is able to visualise more than three objectives by creating an array of the plot projecting all the pairwise combinations of coordinates, the complexity of the information provided to the decision maker increases significantly with the number of objectives (Gao et al., 2019).

- Bubble chart. Bubble chart is another variant of three-dimensional scatter plot which can add additional two dimensions which are visualised by size and colour of the scatter points. The main advantage of bubble chart compares to scatter plot matrix is that it can project at most five objectives in a single plot (Tušar, 2014).

- Parallel coordinates plot. One of the most regularly used techniques for EMO to visualise distribution, range and trade-off among solutions is parallel coordinates plot (Inselberg, 2009). This technique projects a m-dimensional objective into a polyline in the coordinate system with $\mathrm{m}$ parallel axes placing along $\mathrm{x}$-axis. It should be noted that each polyline denotes for a solution for all objectives which the corresponding value can be obtained from the intersection at each parallel axis. Parallel coordinates plot is a very advantageous technique to present dependencies between objectives; however, it becomes difficult to interpret and analyse large sets of data due to the cluttering effect with crossing lines (Gao et al., 2019).

- Heatmap. Another popular technique for high-dimensional data is heatmap which projects the objective value into a colour map. Similar to parallel coordinates plot, it has advantages on 
presenting dependencies between objectives but has the same cluttering problem (Gao et al., 2019).

- Self-organising map. Kohonen (1990) developed a visualisation technique, namely selforganising map (SOM), to specify a mapping from multi-dimensional objective to a lower dimensional space (usually two-dimensional space) through artificial neural networks. In this technique, the input data vectors are mapped to nodes (called neurons) with a weight vector under same dimension. A hexagonal or rectangular grid in a two-dimensional space can be used as the arrangement of the neurons. The unified distance matrix (U-Matrix) is commonly used in SOM to present the distance between adjacent neurons in different colours: dark areas represent cluster boundaries and light areas show clusters of similar neurons.

- Level diagram. Blasco et al. (2008) proposed a visualisation technique called level diagram to plot the approximation sets in an objective-wise manner. In particular, a set of $m$ level diagrams are generated, where $m$ equals to the number of objectives. In each diagram, $\mathrm{x}$-axis represents the objective value at the corresponding objective and y-axis serves as the distance to the ideal objective vector. The authors claimed that this technique is capable to investigate many typical features of Pareto Front including closeness to ideal point, discontinuities and ranges of attainable values. However, it becomes difficult to analyse as the increasing number of objectives can result in significant number of subplots (Gao et al., 2019).

Figure 3.2 demonstrates some Pareto Front visualisation technique examples for the classic DTLZ4 test problems (Ibrahim et al., 2016). More details about the test can be found in (Deb et al., (2005). Figures 3.2 (a) to (e) respectively present a scatter plot matrix for approximate solutions obtained for 4-objective DTLZ4 problem, a bubble chart plot to represent 3dimensional values of 4-objective DTLZ4 problem, a heatmap plot showing approximate solutions obtained for 5-objective DTLZ2 problem, a parallel coordinates plot for 10-objective DTLZ4 test problem and a self-organizing map plot for approximate solutions obtained for 5objective DTLZ4 problem. 


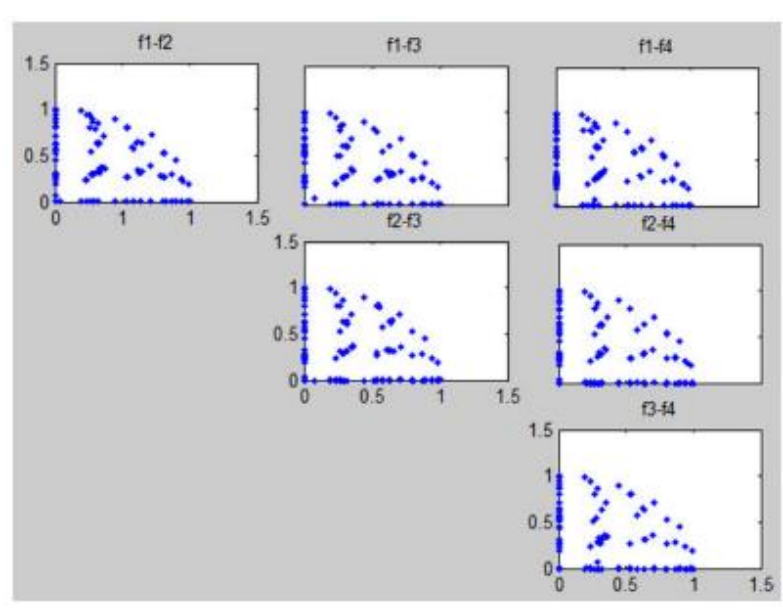

(a) Scatter plot matrix

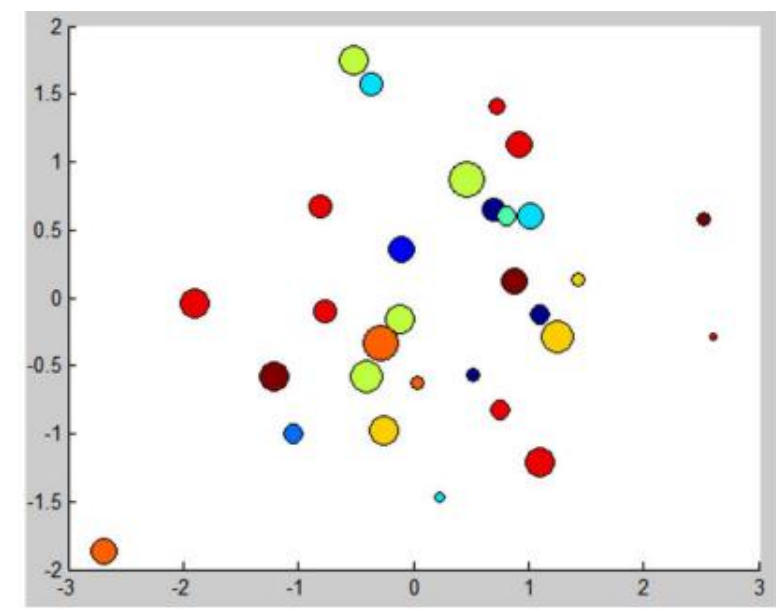

(b) Bubble chart

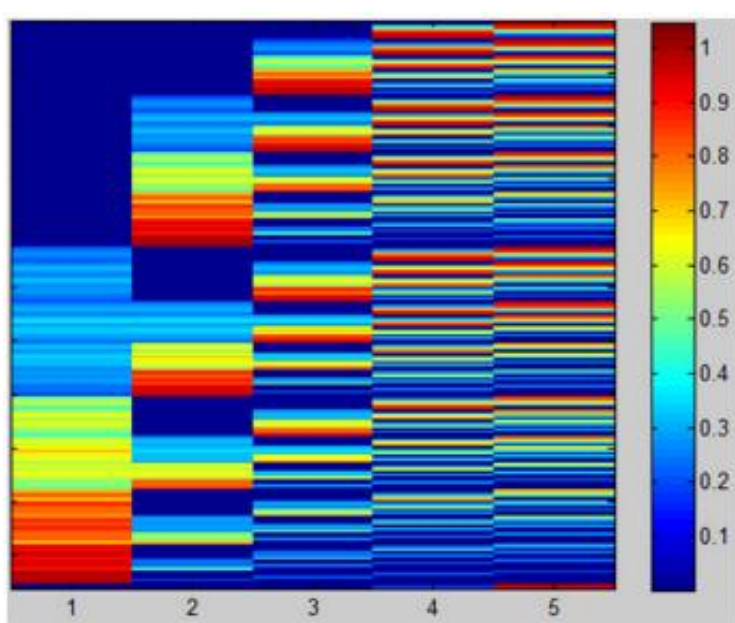

(c) Heatmap

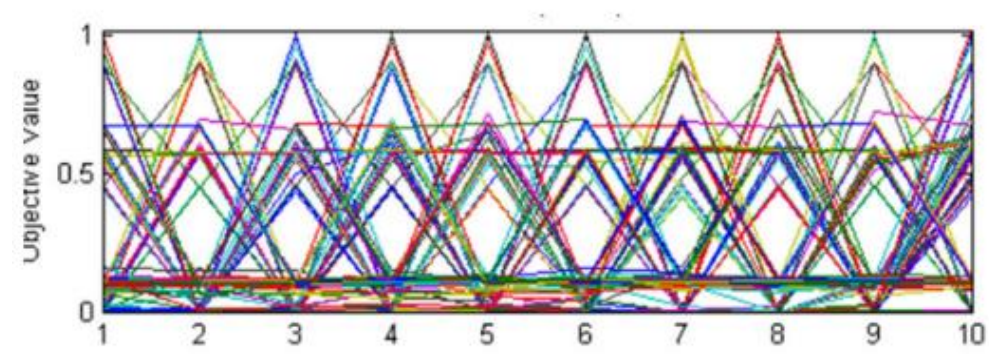

(d) Parallel coordinates plot

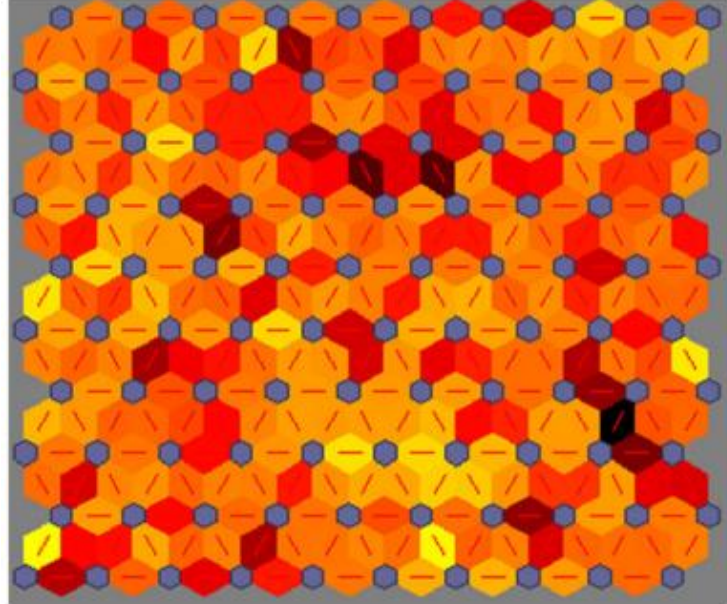

(e) Self-organizing map

Figure 3.2: Visualisation technique examples used in multi-objective optimisation problems for the classic DTLZ4 test problems. (a) Scatter plot matrix. (b) Bubble chart. (c) Heatmap. (d) Parallel coordinates plot. (e) Self-organizing map (Ibrahim et al., 2016). 


\subsection{Applications of Multi-objective Optimisation Methods}

In the preceding section, the relevant studies focusing on the applications of multi-objective optimisation methods in pipeline network design and operation are presented.

\subsubsection{Brown et al. (2014)}

Brown et al. (2014) presented a multi-objective optimisation methodology for optimising Remote Control Valve (RCV) spacing along $\mathrm{CO}_{2}$ pipelines as a trade-off between the reduction in hazard against the valve installation and maintenance cost. The HEM model developed by Mahgerefteh et al. (1999) was employed for predicting the fluid flow parameters following pipeline failure. The optimisation problem is summarised in the following mathematical form:

$$
\min _{d \in D} J_{1}(d), J_{2}(d)
$$

where, $d$ is the spacing between RCVs $(\mathrm{km}) . J_{1}(d)$, the metric defined for the hazard associated with a pipeline failure, is determined as the area bounded by the $7 \%$ (v./v.) concentration contour of the dispersing $\mathrm{CO}_{2}$ cloud. $J_{2}(d)$ is the RCV installation and maintenance costs, calculated by following expression (Medina et al., 2012):

$$
J_{2}(d)=\frac{V_{P N} r(1+r)^{n} L}{\left((1+r)^{n+1}-1\right) d}
$$

Here, $V_{P N}$ is the single RCV cost $(€), r$ is the discount rate, $n$ is the average lifetime of the equipment (y) and $L$ is the overall length of the pipeline $(\mathrm{km})$. For the RCV used in the study, the values used were $€ V_{P N}=15556, r=0.035$ and $n=10$. The valve spacing, $d$ lay in the interval $D=[5,40] \mathrm{km}$.

The above methodology was applied to a hypothetical $96 \mathrm{~km}$ pipeline $(571.2 \mathrm{~mm}$ i.d. with 19.4 mm wall thickness) transporting dense phase $\mathrm{CO}_{2}$. FBR was assumed to occur at the middle of the pipeline. The initial pressure and temperature were respectively at 151 bar and $303.15 \mathrm{~K}$. The RCVs were assumed to close $900 \mathrm{~s}$ following the pipeline failure at a closure rate of 1.90 
$\mathrm{cm} / \mathrm{s}$. Figure 3.3 shows the $7 \%$ (v./v.) concentration contours, representing the shape of the cloud in a horizontal half-pane at a height $1 \mathrm{~m}$ above the ground, as calculated at various time after the beginning of the release. Figures 3.3 (a) and (b) respectively show the variation of $J_{1}$ and $J_{2}$ as a function of valve spacing and the Pareto set of the optimisation problem.

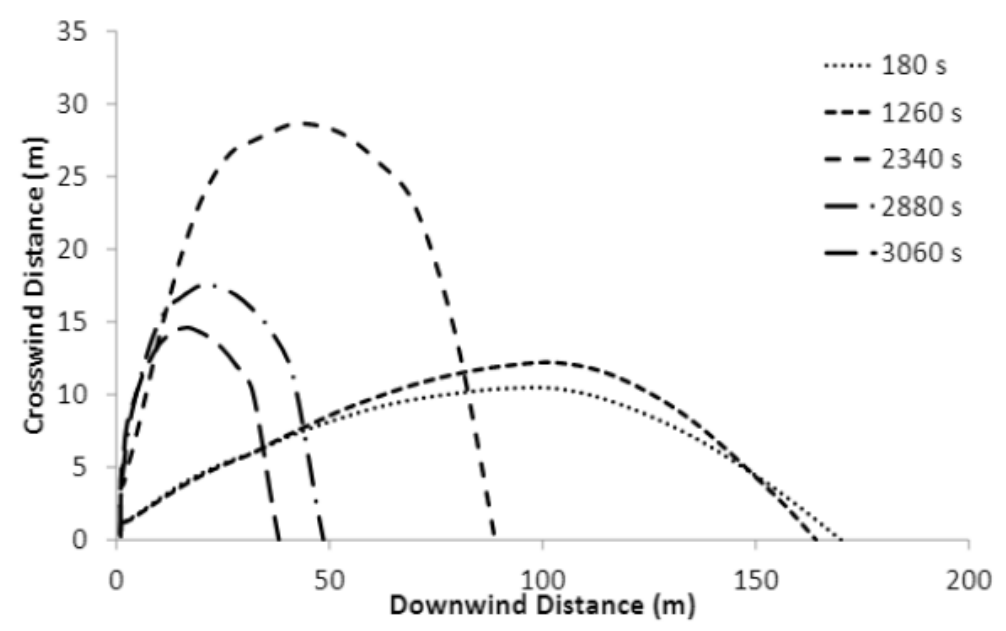

Figure 3.3: Variation of $7 \%$ (v./v.) concentration half - contours with time (Brown et al., 2014).

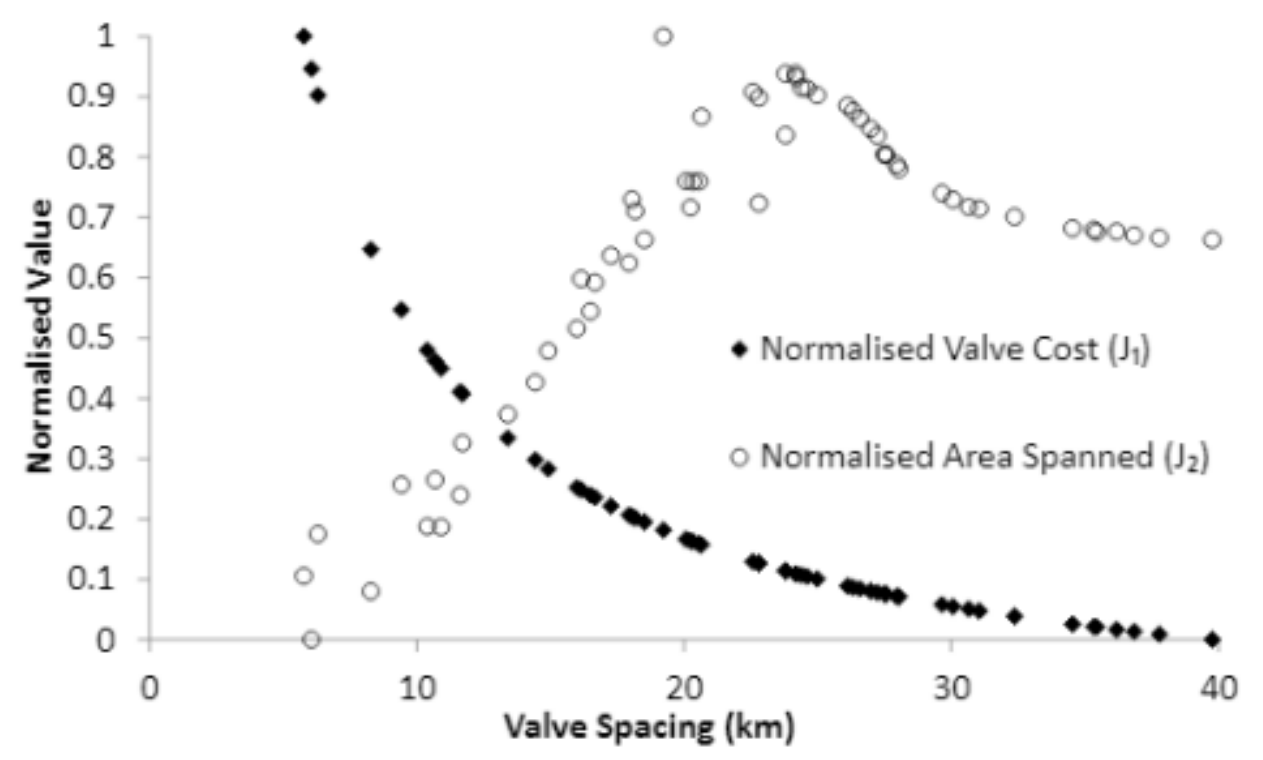

(a) 


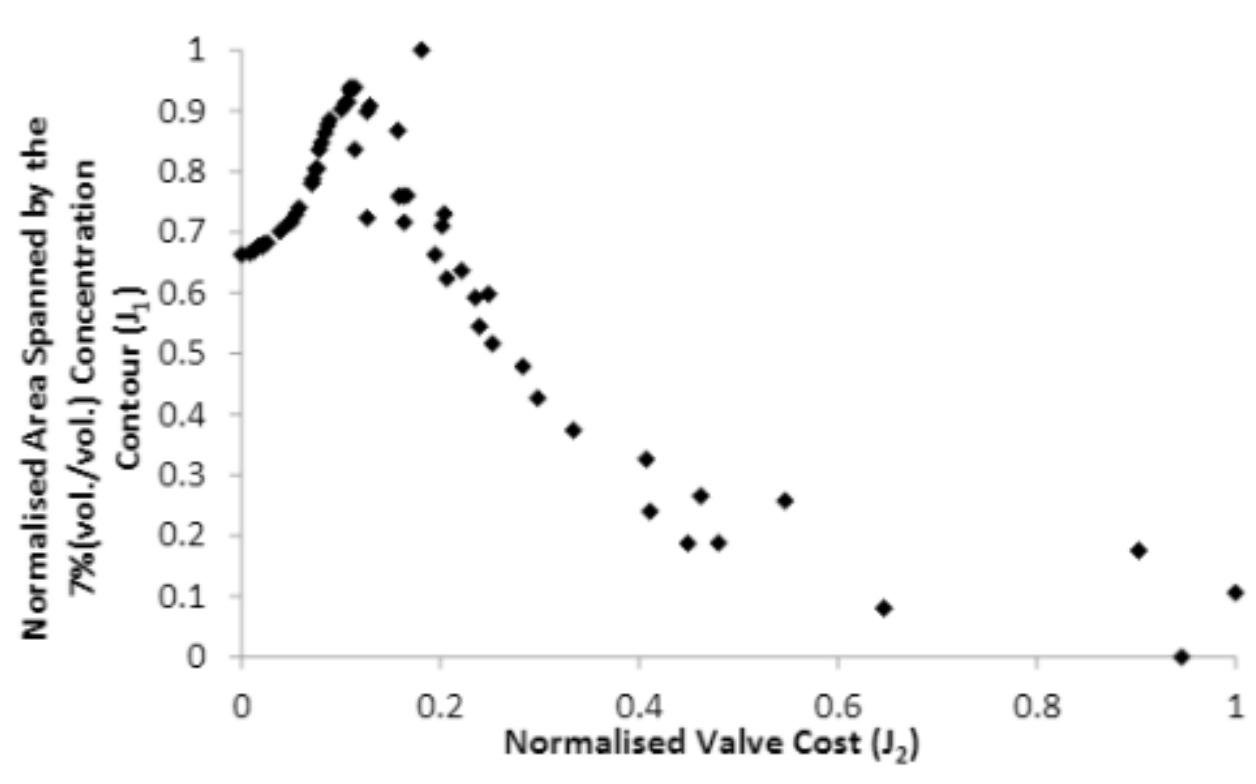

(b)

Figure 3.4: Valve spacing multi-objective optimisation results: (a) Normalised valve cost and area spanned by $7 \%$ (v./v.) concentration as a function of valve spacing (b) Pareto set of the problem (Brown et al., 2014).

Referring to figure 3.3, the dimensions of the cloud vary significantly during the initial period of release. More specifically, during the times from 180 to $2340 \mathrm{~s}$ the half width of the cloud increases from ca. 10 to $30 \mathrm{~m}$, while its downwind length decreases from ca. 170 to $90 \mathrm{~m}$. After ca. $2340 \mathrm{~s}$ the area of the cloud bounded by $7 \%$ (v./v.) concentration contour is gradually shrinking with time. As can be observed in figure 3.4 (a), the normalised cost of the valves shows a hyperbolic decrease as the valve spacing is increased. In contrast, the normalised area spanned shows a near linear increase from 0 to 1 as the valve spacing increases from ca. 5 to $24 \mathrm{~km}$. As the valve spacing increases beyond $24 \mathrm{~km}$, the normalised area falls to a near constant value of ca. 0.7. The authors explained that the latter is not due a real effect but a result of the model integration which leads to the numerical noise observed throughout. From figure 3.4 (b), the variation of the normalised area spanned is observed to increase at normalised costs of 0 and ca. 0.1 . Following this, the normalised area decreases hyperbolically to 0 from a normalised area of 1 . The implication of these results is that the hazard reduction obtained by increasing the number of valves becomes marginal above a certain range. 


\subsubsection{Demissie (2015)}

Demissie (2015) developed a multi-objective optimisation method to optimise the operation of natural gas pipeline networks. The purpose of the study was to find the optimal pipeline and compressor constraints (i.e. pressure at the nodes and the rotational speed of the compressors) to minimise power consumption while maximising the gas delivery flow rate of the system. Three topologies namely linear, branched and looped was applied for the network configurations. An empirical steady-state isothermal pipeline flow equation was used to calculate the pressure drop in the pipeline and Nikuradse's equation was implemented to estimate the frictional factor. The power required to compress the gas in the compressor was calculated based on the reversible adiabatic compression assumption. The optimisation problem associated with two objective functions is summarised in the mathematical form below (Demissie, 2015):

Maximise $\sum b_{\text {out }}$

Minimise $K \sum \frac{b_{\text {out }} H}{\eta}$

where, equations 3.3 and 3.4 respectively represent maximising the gas delivery flow rate of the system and minimising the power consumption of compressor stations. $b_{\text {out }}, K, H$, and $\eta$ are the gas delivery flow rate, isentropic exponent, compressor head and efficiency respectively.

Three case studies which were firstly presented by $\mathrm{Wu}$ (1998) were introduced with some modifications for the optimisation study to consider all linear, branched and looped topologies. The full details of the case studies set up can be found in $\mathrm{Wu}$ (1998). The following is a summary of the main modifications adopted by Demissie (2015).

Figures 3.5 to 3.7 respective show the schematic representation for the case studies 1 to 3 . Here, CS, L, D and b are respectively compressor station, pipeline length, pipeline outside diameter and supply/delivery node. The minimum and maximum values of the speed of the compressors; 
the volumetric flow rates of the compressors; the pressure limits at the nodes were respectively $5000 \mathrm{rpm}$ and $9400 \mathrm{rpm} ; 7000 \mathrm{ft}^{3} / \mathrm{min}$ and $22000 \mathrm{ft}^{3} / \mathrm{min} ; 600$ and $800 \mathrm{psia}$.

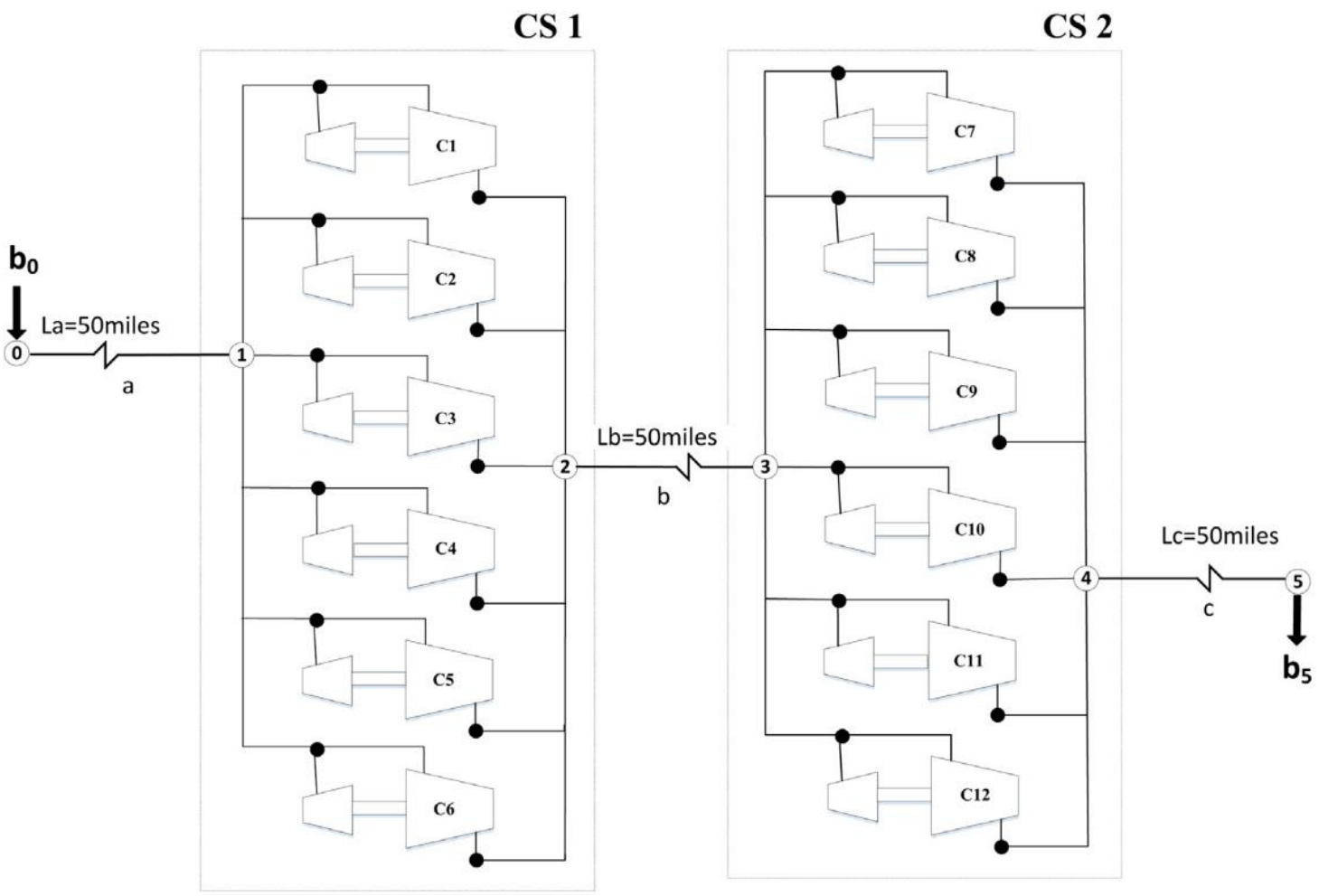

Figure 3.5: Schematic representation of case study 1: linear network topology (Demissie, 2015). 


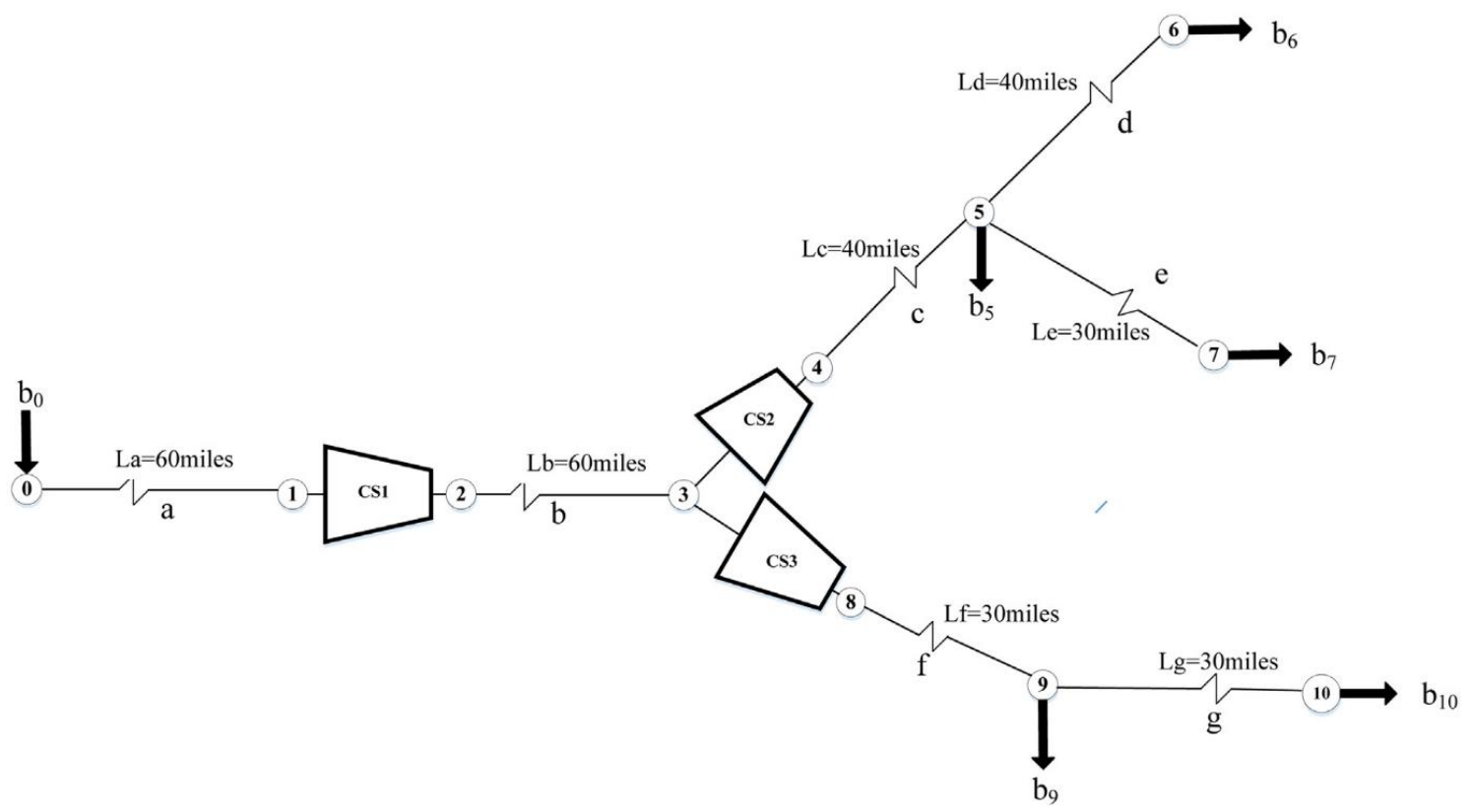

Figure 3.6: Schematic representation of case study 2: branched network topology (Demissie, 2015).

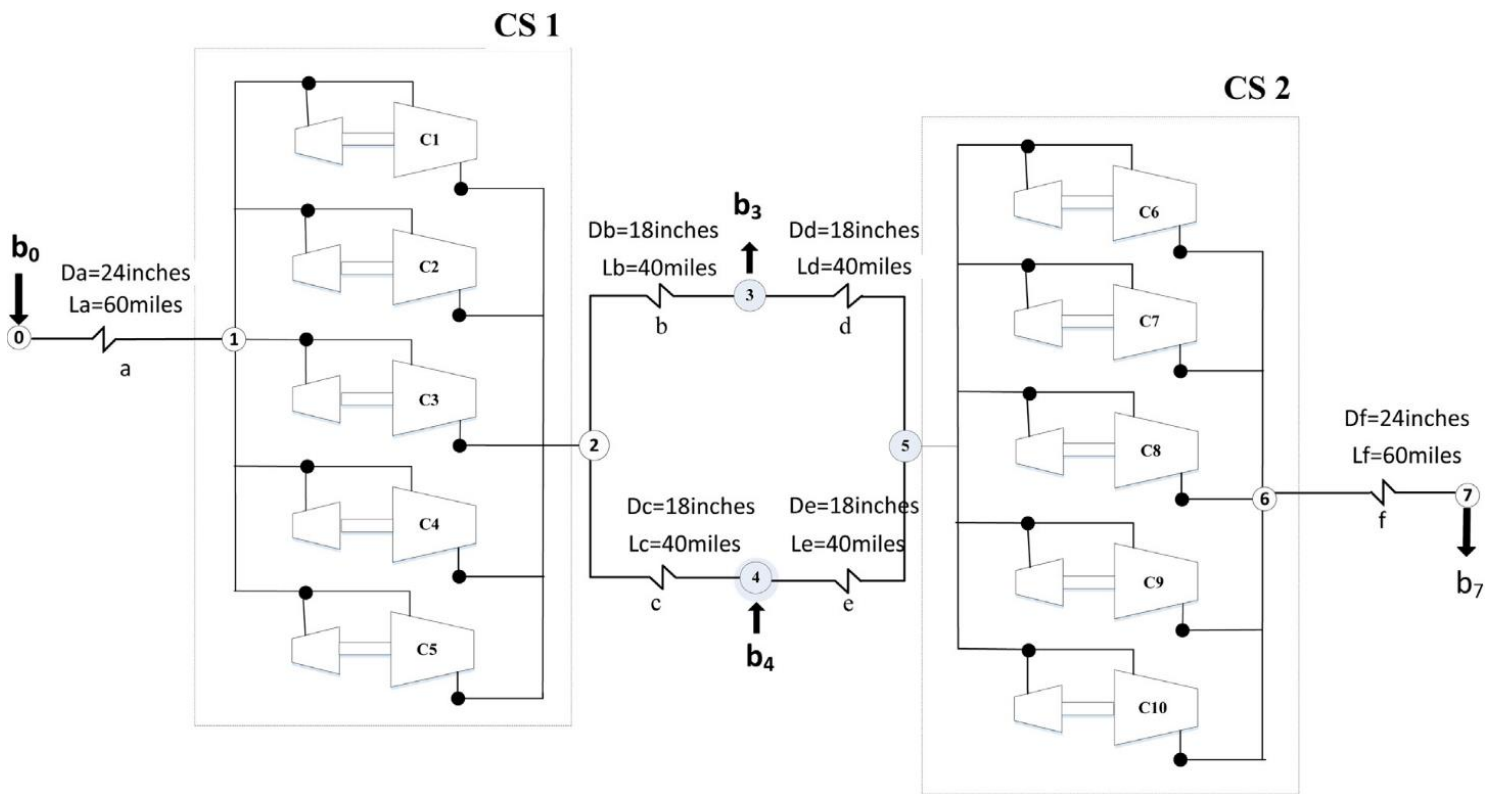

Figure 3.7: Schematic representation of case study 3: looped network topology (Demissie, 2015).

Table 3.1 shows the composition of natural gas and its thermodynamic properties. 
Table 3.1: Feed composition and component heat capacity at constant pressure (Demissie, 2015).

\begin{tabular}{|cccc|}
\hline Component & Methane & Ethane & Nitrogen \\
\hline Mole percent & 85 & 14 & 1 \\
Heat capacity at constant pressure & 0.59 & 0.39 & 0.25 \\
\hline
\end{tabular}

Figures 3.8 to 3.10 respectively show the optimum points for minimising power consumption and maximising delivery flow rate for case studies 1 to 3 .

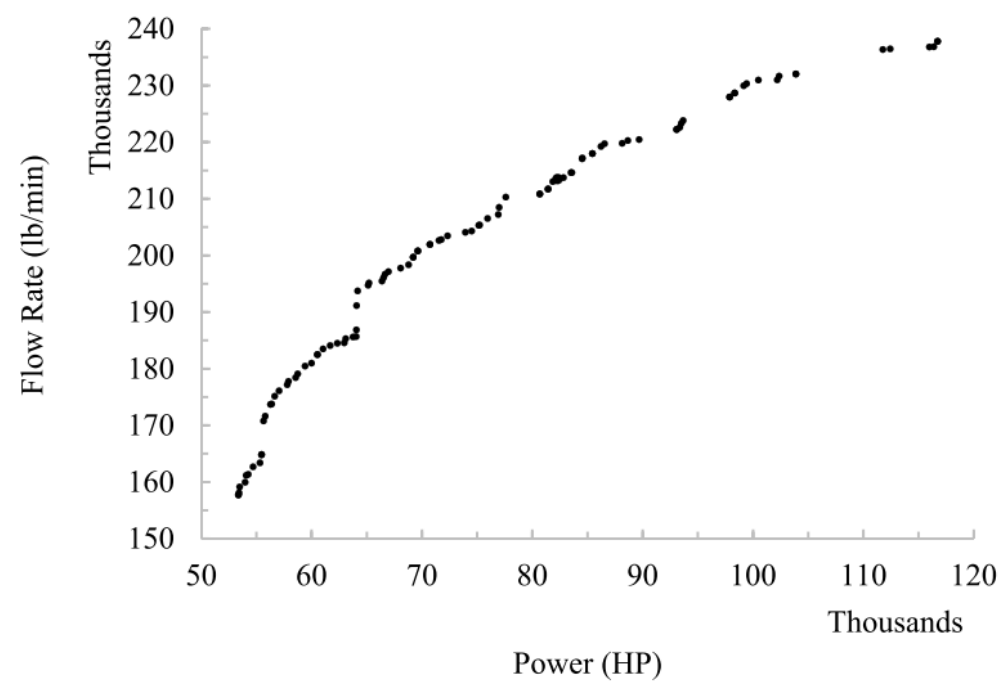

Figure 3.8: Optimum points for minimising power consumption and maximising delivery flow rate for case study 1: linear network topology (Demissie, 2015). 


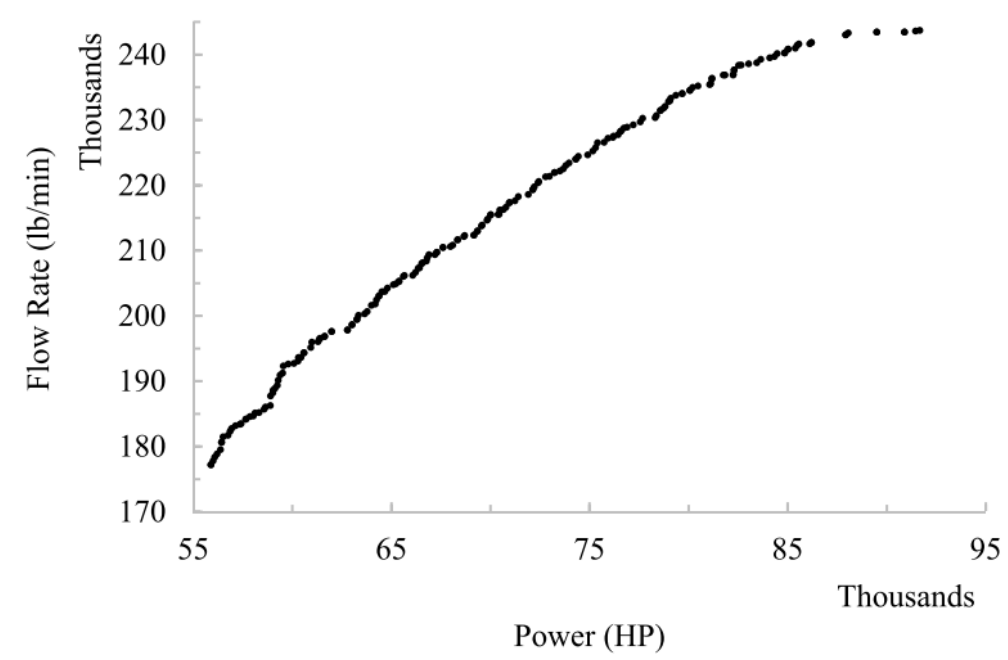

Figure 3.9: Optimum points for minimising power consumption and maximising delivery flow rate for case study 2: branched network topology (Demissie, 2015).

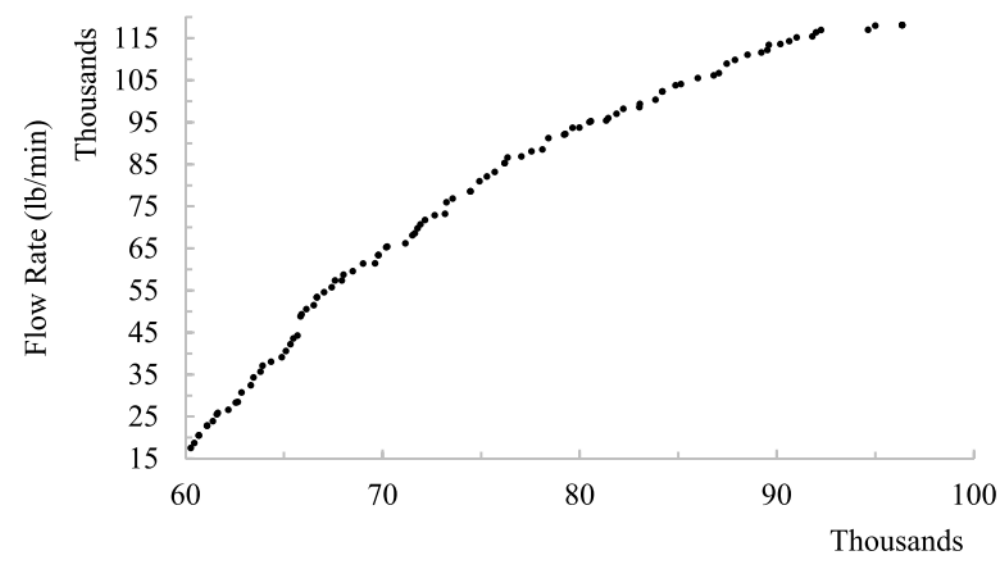

Figure 3.10: Optimum points for minimising power consumption and maximising delivery flow rate for case study 3: looped network topology (Demissie, 2015).

As can be observed from figures 3.8 to 3.10, for all three case studies, with the increase of the power consumptions, delivery flow rates show a nearly logarithmic growth. The authors explained that each Pareto optimal point is an acceptable solution where the decision maker need to choose a single solution based on his knowledge of the operation of the networks and the associated costs. During the selection process, the decision maker may always review the chosen solution incorporating practical information and experience to identify if the solution 
has significantly improvement for system operation. The above finding highlights the capability of applying multi-objective optimisation in providing a set of optimal network operation of natural gas pipeline network.

\subsubsection{Alves et al. (2016)}

Alves et al. (2016) proposed a multi-objective optimisation method in the design of natural gas transmission networks in order to minimise the transportation fare while maximising the transported gas volume. For the optimisation problem, various of optimisation variables were considered including the design variables of pipe sections and compression stations, costs and operation conditions as presented in table 3.2.

Table 3.2: Optimisation variables (Alves et al., 2016).

\begin{tabular}{|ll|}
\hline Variables Description & Unit \\
\hline Variables for each pipe section & \\
Cross sectional area of pipe & $\mathrm{m}^{2}$ \\
Outer diameter & $\mathrm{m}$ \\
Thickness & $\mathrm{m}$ \\
Darcy friction factor & - \\
Length & $\mathrm{km}$ \\
Mass of steel & $\mathrm{kg}$ \\
Economy of scale term & $\mathrm{MM} \mathrm{USD}$ \\
Pipeline miscellaneous costs & $\mathrm{MM} \mathrm{USD}$ \\
Pipeline right if way costs & $\mathrm{MM} \mathrm{USD}$ \\
Pipeline material costs & $\mathrm{MM} \mathrm{USD}$ \\
Pipeline construction labour costs & $\mathrm{MM} \mathrm{USD}$ \\
\hline Variables for each compression stations & \\
Total cost of compression stations & $\mathrm{MM} \mathrm{USD}$ \\
Mean operational power for the year & $\mathrm{HP}$ \\
Design power & $\mathrm{HP}$ \\
\hline Variables for each edge & \\
Capital investment & $\mathrm{MM} \mathrm{USD}$ \\
Flow rate for the year & $\mathrm{MM} \mathrm{Nm}{ }^{3} / \mathrm{day}$ \\
\hline
\end{tabular}




\begin{tabular}{|ll|}
\hline Variables for each node & \\
$\begin{array}{l}\text { Absolute pressure for the year } \\
\text { External flow rate for the year }\end{array}$ & $\mathrm{bar}$ \\
\hline Economic variables for each year & \\
Cash flow & $\mathrm{MM} \mathrm{US} /$ day \\
Operational costs with transportation & MM USD \\
Transportation revenues & MM USD \\
\hline Other variables & \\
Outer diameter of branch & $\mathrm{m}$ \\
Present value of the cash flows & MM USD \\
Transportation fare & USD/MM BTU \\
Net present value & MM USD \\
\hline
\end{tabular}

The $\varepsilon$-constraint method (Marler and Arora, 2004) was applied to conduct the Pareto set and the problem constraints were determined as: mass balance and delivery, pipeline design, compression station design and economic. The optimisation problem for the design of a natural gas transportation is defined below (Alves et al., 2016):

$$
\left\{\begin{array}{cc}
\min _{x} & \Lambda(\boldsymbol{x}) \\
\max _{x} & \mathrm{~V}(\boldsymbol{x}) \\
\text { subject to } & \boldsymbol{h}(\boldsymbol{x})=\mathbf{0} \\
& \boldsymbol{g}(\boldsymbol{x}) \leq \mathbf{0}
\end{array}\right.
$$

where, $\boldsymbol{x}$ is the vector of optimization variables. The objective functions $\Lambda(\boldsymbol{x})$ and $\mathrm{V}(\boldsymbol{x})$ are respectively the transportation fare and total gas volume delivered during all the years of operation. $\boldsymbol{h}(\boldsymbol{x})$ and $\boldsymbol{g}(\boldsymbol{x})$ are vectors of constrains. The $V(\boldsymbol{x})$ function can be calculated by following expression:

$$
\mathrm{V}(\boldsymbol{x})=-\frac{D_{O P}}{1000} \sum_{n \in P D} \sum_{\tau \in T I} w_{\tau, n}
$$

where, $\tau \in T I$ is the index of the operation year. $D_{O P}$ is the number of operational days per year and $w_{\tau, n}$ is the flow rate in $\mathrm{MM} \mathrm{Nm}^{3} /$ day of node $n$ during the year $\tau$. 
The methodology was applied to a case study of a hypothesis natural gas transportation network which the trunk line is composed of a single source and a single sink with three compression stations. The schematic representation of the case study is presented in figure 3.11. The pipe section, compression station, network inlet node, suction and discharge nodes of the compression stations are denoted by PI, CO, PS, SUC and DISC, respectively. The flow rates at edges and external flow rates at nodes are respectively presented by $\mathrm{q}$ and $\mathrm{w}$.

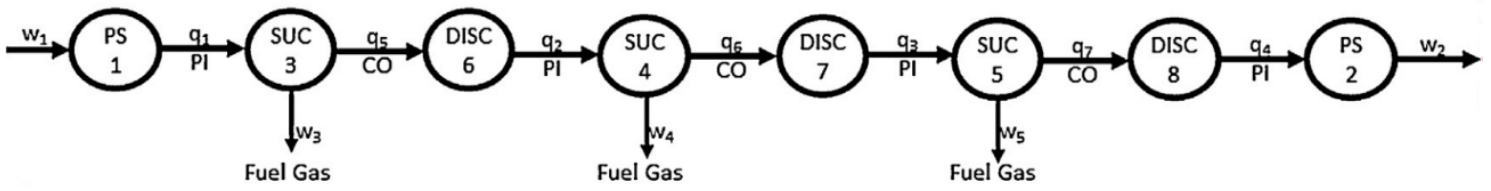

Figure 3.11: Schematic representation of the natural gas transportation network (Alves et al., 2016).

The simulation results for Pareto front are presented in figure 3.12, where the $\mathrm{x}$-axis and $\mathrm{y}$-axis respectively represent the delivered gas volume and the transportation fare. Example points (a), (b), (c), (d), (e) and (max) were highlighted by the authors for the different optimal solutions for the proposed problem. Points (a) and (max) respectively represent the design that gives the minimum fare and the design with the delivered volume equal to the total available demand. The point $(\mathrm{x})$ presents the design in the feasible region (hatched area) which does not represent an optimal solution. 


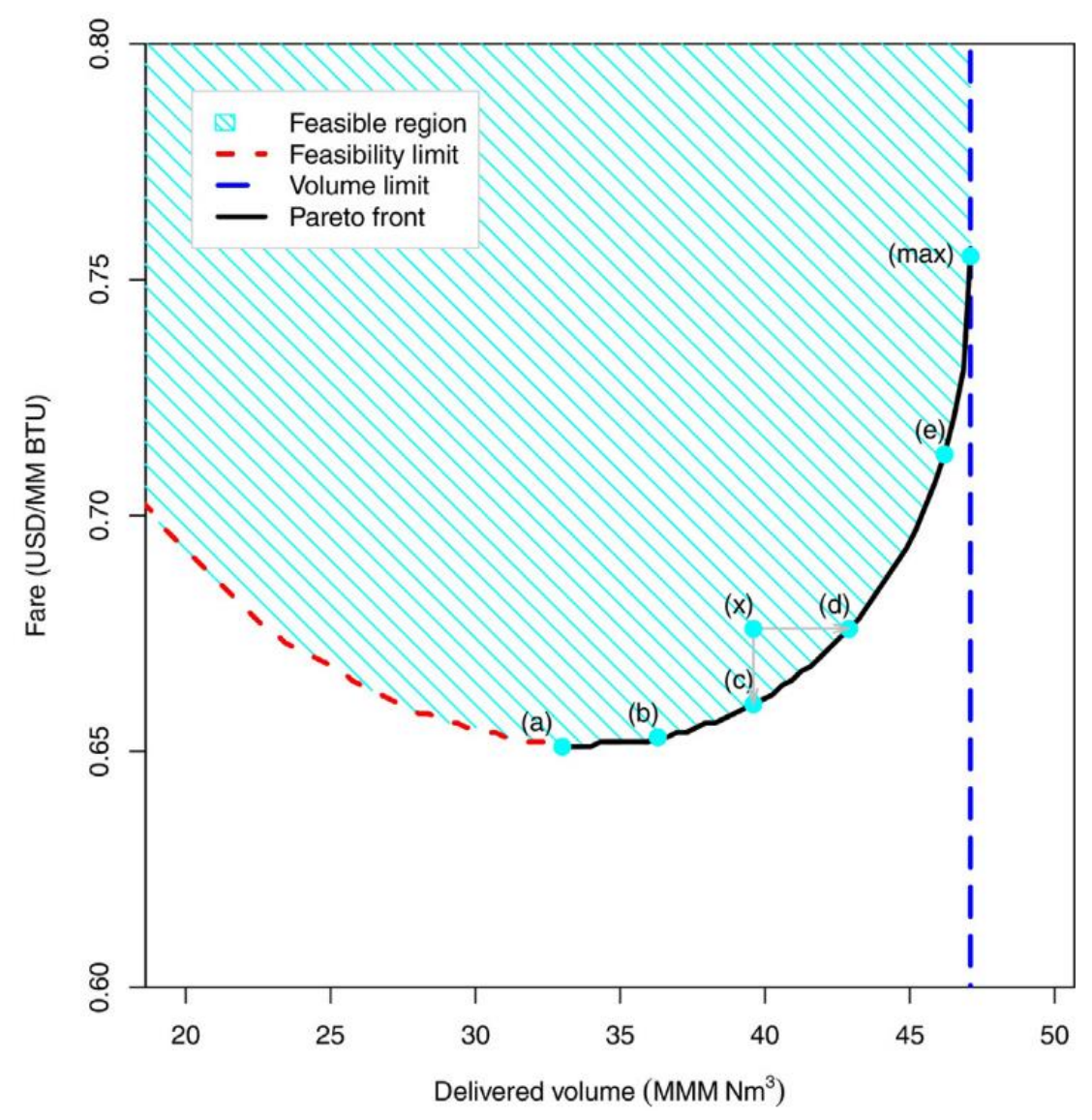

Figure 3.12: Pareto front: the variation of the transportation fare as a function of the delivered gas volume (Alves et al., 2016).

As may be observed in the figure 3.12, the solution of the Pareto front is marked in black line which shows an exponential growth for the transportation fare while the delivered volume increases from ca. $33 \mathrm{MMM} \mathrm{Nm}^{3}$ (million million normal cubic meters, equivalent to $10^{12}$ $\mathrm{Nm}^{3}$ ) to ca. $47 \mathrm{MMM} \mathrm{Nm}^{3}$. The Pareto front can be used to identify the optimal solution by finding the best balance between the amount of gas delivered to the population and the transportation fare.

It should be pointed out here that the handling of the computational runtime becomes a more sensitive problem when considering a large number of optimisation variables. 


\subsection{Concluding Remarks}

This chapter presented a review of the development of multi-objective optimisation methods. The general approaches for selecting the optimal solutions and the specific techniques to visualise the Pareto Front were first discussed. This was followed by a survey of the relevant studies focusing on the method applications. Based on the above review, it is clear that considerable progress has been made in applying multi-objective optimisation methods in providing a set of optimal solution in pipeline network design and operation.

\section{Gaps in knowledge:}

Despite the progress, significant gaps in developing and applying a multi-objective optimisation method for selecting optimal inline ESDV configurations in high-pressure pipelines exists.

For example, very few studies can be found on the application of such methods to inline ESDV pipeline configurations. In a recent attempt, Brown et al. (2014) presented a multi-objective optimisation method to optimise valve spacing in $\mathrm{CO}_{2}$ pipelines as a trade-off between the reduction in risk against the cost in ESDV installation and maintenance. Nevertheless, with the exception of the valve spacing, the impact of other important optimisation variables for ESDV configuration such as ESDV type \& their combination, number and operational settings (e.g. activation \& closure times) were not considered.

Additionally, for the purpose of assessing the impact of pipeline component failure, another essential consideration in practice is the ESDV reliability estimation such as the failure rates. The ESDV failure rates can be used for demonstrating the feasibility of the risk reduction being targeted by safety functions, which is extremely important in optimising ESDV configuration. As such, the ESDV failure rates should be considered as an objective function along with the reduction in risk and the ESDV capital cost.

Unfortunately, increasing the number of optimisation variables or objective functions leads to significant additional mathematical complexities, making solutions difficult to visualise and analyse. To overcome this limitation, appropriate variable reduction techniques may be applied to identify the redundant variables that can be disregarded whilst still ensuring an improved outcome. 


\section{Aim and objectives of the study:}

To address the challenges and the gaps on knowledge presented in this chapter and Chapter 2 (Section 2.7), the aim of this study is to develop and apply a multi-objective optimisation method for selecting the ESDV type, number and spacing as well as its combinations and operational settings for striking a balance among three objective functions namely:

iv. the ESDV capital cost

v. the ESDVs failure rate

vi. the amount of total inventory escaping prior to complete valve closure following pipeline failure

Based on modelling of the in-pipe transient fluid flow behaviour using the HEM model, the main project objectives are to:

- simulate and investigate ESDV dynamic response following pipeline failures

- simulate and analyse the efficacy of ESDVs based on their type, number, spacing and combination in minimising and ultimately isolating outflow following pipeline failures

- develop and apply using a real high-pressure transmission pipeline as a case study, a multi-objective optimisation method combined with PCA for determining the optimal ESDV configurations for high-pressure pipelines

In the proceeding chapter, the HEM model described in Chapter 2 is applied to investigate ESDV dynamic response and characteristics on the fluid behaviour following the accidental failure of high-pressure pipelines. 


\section{Chapter 4:}

\section{Modelling and Study of Emergency Shutdown Valve Dynamic Response Following Accidental Failure of High-Pressure Pipelines}

\subsection{Introduction}

As discussed in Chapter 1, the failure of high-pressure pipelines transporting large amounts of hazardous fluids poses significant safety concerns. In order to minimise the associated risks, Emergency Shutdown Valves (ESDVs) may be installed along their length in order to minimise and ultimately isolate the amount of the escaping fluid.

The type and the optimum strategic positioning of such valves, along with their number, striking a balance between risk reduction against the valve capital cost requires a knowledge of the in-pipe flow dynamics during valve closure. The fluid flow dynamics may be obtained using the Homogeneous Equilibrium Mixture (HEM) model described in Chapter 2. The subsequent fluid flow behaviour upon and during valve closure on the other hand may be simulated through the implementation of appropriate boundary conditions (Section 2.5, Chapter 2).

The purpose of this chapter is to model, compare and contrast the dynamic response of different types of ESDV's following the accidental failure of high-pressure pipelines. Ethylene is chosen as a case example of the fluid being transported, given its hazardous nature (highly flammable and explosive) along with its extensive use in the petrochemical industry as a feedstock in the manufacture of polymers such as polyethylene (PE), polyethylene terephthalate (PET), polyvinyl chloride (PVC) and polystyrene (PS) (Deopura et al., 2008). The results presented include the simulation and discussion of pressure surges upon Check Valve (CV) closure and the impact of Automatic Shut-off Valve (ASV) and Remote Control Valve (RCV) activation pressure on their efficacy in limiting the amount of the total inventory loss prior to complete valve closure.

The chapter is organised as follows: in Section 4.2, data relating to the simulations of the rapid closure of a CV installed in a hypothetical nevertheless realistic ethylene pipeline following its 
Full Bore Rupture (FBR) is presented and discussed. The resulting pressure surges are analysed in detail with a particular focus on the impact of the ethylene pipeline operating pressures and temperatures. In Section 4.3, the impact of ASV and RCV activation pressure is investigated by predicting the amount of total inventory loss prior to complete isolation following FBR and puncture failures of the ethylene pipeline. Such data is of paramount importance as it can serve as the source term for determining the consequences of pipeline failures that include fire, explosions and toxic release. These ultimately dictate the minimum safe distances to populated areas and emergency response planning. Conclusions are drawn in Section 4.4.

\subsection{The Study of Pressure Surges upon CV Closure}

In practice, the near-instantaneous closure of a $\mathrm{CV}$ upon rupture may produce a pressure surge which, could in turn create a sufficiently large thrust force acting on segments of the pipeline, resulting in deformation and vibrations of the pipeline (Koetzier et al., 1986; Mahgerefteh et al., 1997; Ord, 2006). In extreme cases, such pressure surges may exceed the maximum safe operating pressure of the pipeline leading to secondary catastrophic failure. Other potential risks include fluid cavitation and momentary opening of the valve due to the very large pressure drop across the closing valve (Koetzier et al., 1986; Ord, 2006). As such, accurate prediction of the pressure surges induced upon closure of CVs are of primarily importance in order to avoid the above risks.

\subsubsection{Simulation Test Setup}

The simulations are performed for a thermally insulated (heat transfer coefficient $=6 \mathrm{~kW} / \mathrm{m}^{2} \mathrm{~K}$ ) $10 \mathrm{~km}$ length, $250 \mathrm{~mm}$ i.d. and $12 \mathrm{~mm}$ wall thickness carbon steel pipeline, representative of a typical ethylene onshore pipeline (Ryder, 1997; Saville et al., 2004; EPS, 2013). The pipe wall roughness, ambient pressure and temperature are assumed to be $0.05 \mathrm{~mm}, 1$ bara and $20{ }^{\circ} \mathrm{C}$ respectively. The upstream and downstream of the pipe are respectively assumed to be represented by an infinite reservoir (constant upstream pressure and mass flow rate) and open end. The pipeline operating pressure and temperature ranges prior to rupture assumed are typical for High-Pressure Ethylene Pipelines (HPEP) and Low-Pressure Ethylene Pipelines 
(LPEP) in various climates. For reference purposes, figure 4.1 shows the ethylene pressuretemperature phase diagram, indicating the HPEP and LPEP operation envelopes (Saville et al., 2004).

The nominal transportation velocity of the ethylene stream is assumed to be $1 \mathrm{~m} / \mathrm{s}$, again, typical of existing ethylene pipelines (IMPEL, 2009; EPS, 2013). For this, and all the subsequent simulations presented in this thesis, 1500 equal-spaced grids with a constant CFL factor of 0.9 are employed as the numerical discretisation procedure.

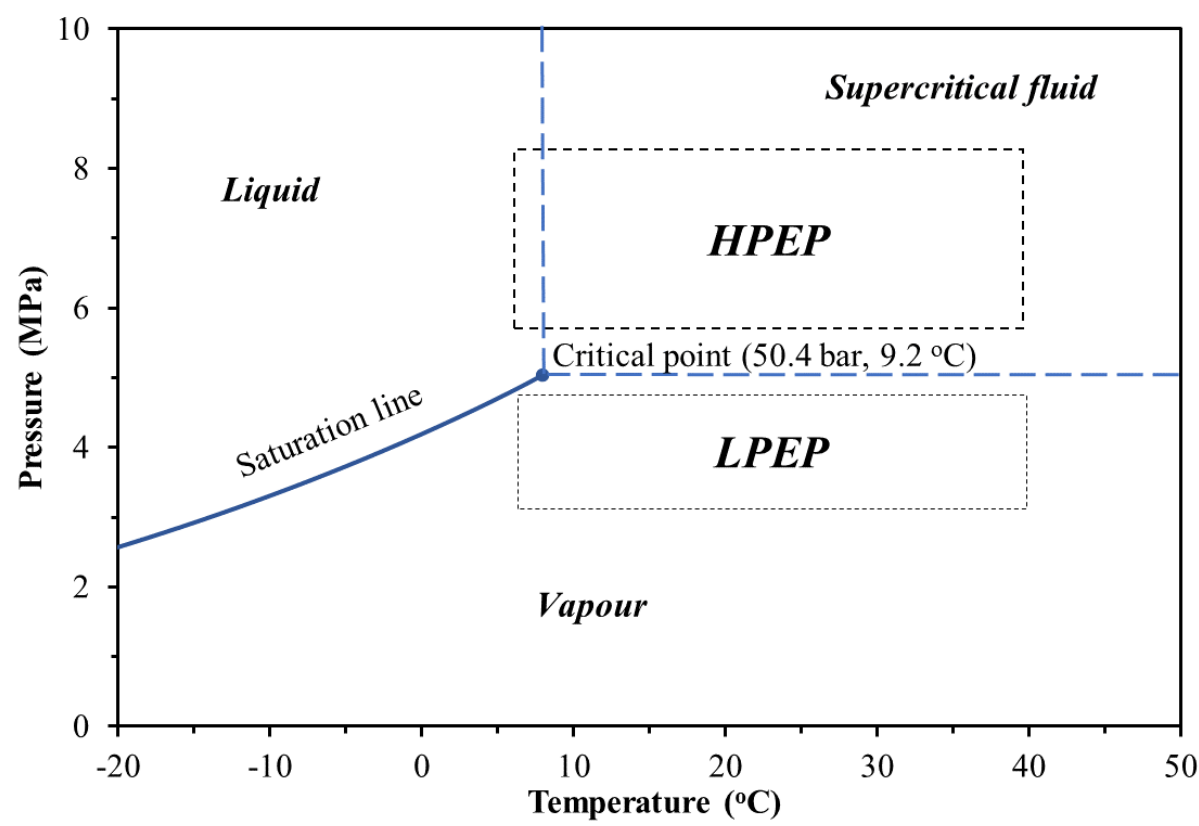

Figure 4.1: Pressure versus temperature phase diagram for ethylene, including the HPEP and LPEP operation ranges.

Figure 4.2 shows a schematic of a section of the ethylene pipeline with FBR located at a distance, $x$ upstream of the $\mathrm{CV}$. In this study, $x$, is set to $300 \mathrm{~m}$, while the distance, $D$ between the $\mathrm{CV}$ and pipe end is taken as $5 \mathrm{~km}$; sufficiently long to ensure no interference from the decompression flow with the boundary conditions at the upstream and downstream ends of the pipe section. This is verified through a number of simulations with varying pipeline lengths. 


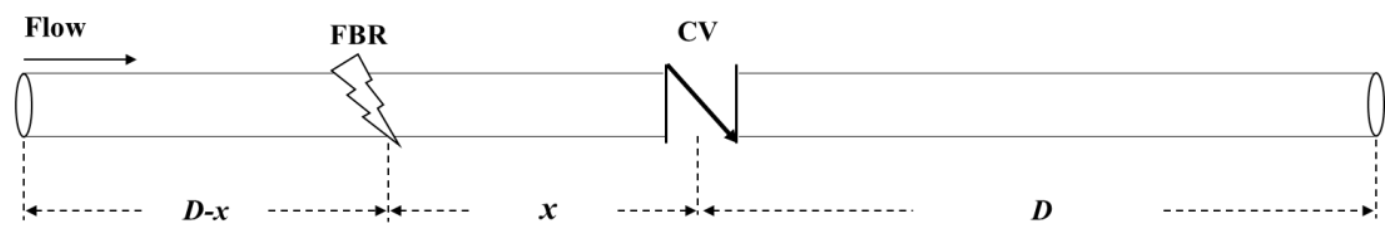

Figure 4.2: Schematic representation of the ethylene pipeline section indicating the location of the $\mathrm{CV}$ relative to the failure point.

As discussed in Chapter 2, a CV ideally closes instantaneously when flow reversal occurs at its location. In practice, valve closure occurs after some level of back-flow is established. In this section, $\mathrm{CV}$ closure time is characterised by the valve closure delay time, $\Delta t_{d}$, spanning the period between the valve activation time, $t_{a}$, upon flow reversal, and its complete closure time, $t_{c}$. In this study, $\Delta t_{d}$ is assumed to be in the range of 0.2 (near-instantaneous closure when flow reversal occurs at its location) to $20 \mathrm{~s}$ (sufficiently long to ensure practical relevance; Ord, 2006). Also, the CV is assumed not to present any obstruction to the escaping fluid until its complete closure. Its subsequent impact on the flow is modelled by the closed end boundary condition (see Section 2.5.5.1, Chapter 2) at the valve location.

\subsubsection{Results and Discussion}

Figure 4.3 shows the simulated pressure variation at the upstream side of CV versus time following FBR for different $\mathrm{CV}$ closure delay times; $\Delta t_{d}$ of $0.2,1.5,9.5$ and $20 \mathrm{~s}$ for HPEP transporting liquid ethylene at 90 bar and $5^{\circ} \mathrm{C}$.

As it may be observed, for all the cases simulated, the valve closure is accompanied by a rapid pressure rise, whose magnitude decreases with the increase in the valve closure delay time. This can be explained by the decrease in the pressure at the valve as a result of the fluid decompression. For closure delay times of $0.2 \mathrm{~s}$ and $1.5 \mathrm{~s}$, the upstream fluid pressure remains relatively constant after the valve closure. While for closure delay times longer than $9.5 \mathrm{~s}$, the pressure downstream of the valve gradually increases after valve closure during the pressure stabilisation. 


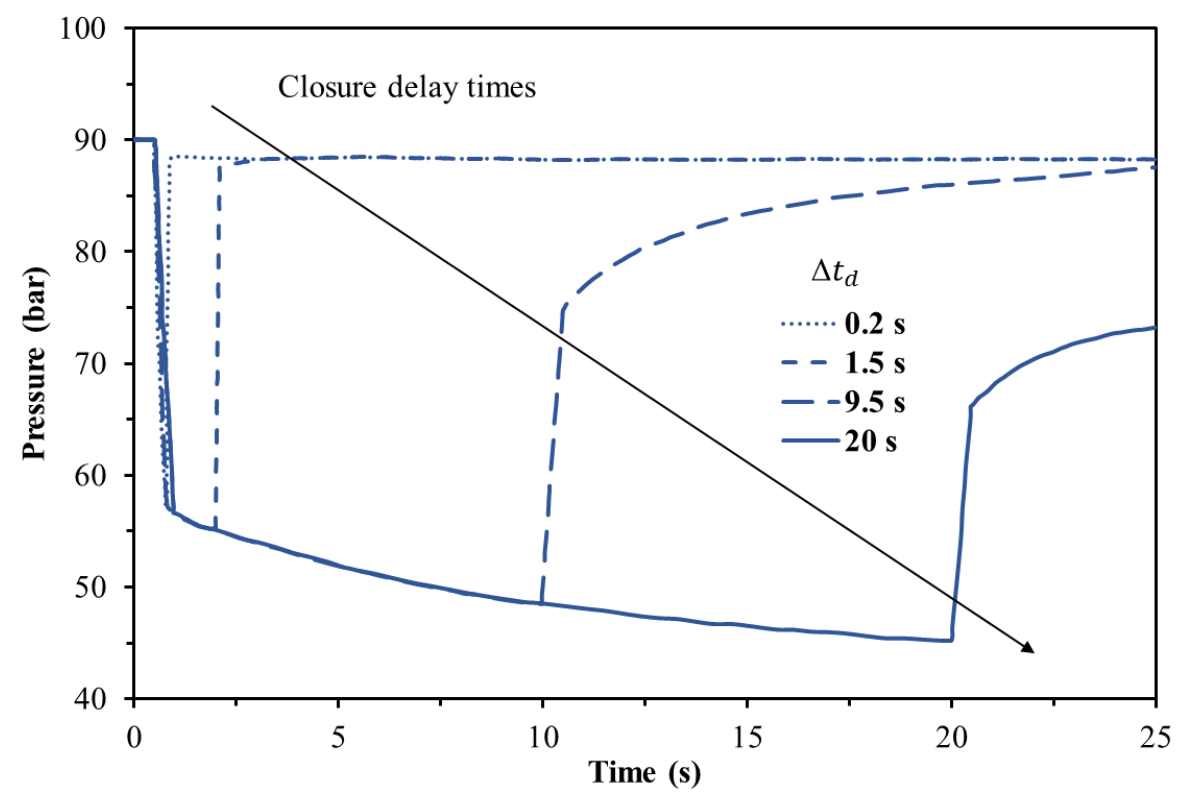

Figure 4.3: Pressure variation with time at the upstream side of CV activated following FBR of a pipeline transporting ethylene at 90 bar and $5{ }^{\circ} \mathrm{C}$, for various valve closure delay times, $\Delta t_{d}$

Assuming instantaneous valve closure, the Joukowsky equation (Muhlbauer, 2004), which takes into consideration the elasticity of the pipe wall and the compressibility of the fluid itself through the calculation of the speed of sound, can be applied to determine the pressure surges, $\Delta p:$

$$
\Delta p=\rho u a
$$

where, $\rho$ and $a$ are respectively the fluid density and the speed of sound, and $u$ is the flow velocity downstream of the surge, corresponding to the fluid velocity prior to the valve closure. $\rho, a$ and $u$ are obtained using the flow model presented in Section 2.3, Chapter 2.

Figure 4.4 shows the effect of the $\mathrm{CV}$ closure delay time on the pressure surge for pipelines transporting supercritical, liquid or vapour phase ethylene operating at $30-90$ bar. The initial temperatures are $5{ }^{\circ} \mathrm{C}$ (liquid phase in HPEP) and $30{ }^{\circ} \mathrm{C}$ (supercritical state in HPEP). As it may be observed, in all cases, as the valve closure delay time increases, the pressure surge increases; with higher magnitude surges obtained at higher operating pressures. Remarkably, 
according to figure $4.4(a)$, in the case where ethylene is transported in liquid phase at 90 bar and $5{ }^{\circ} \mathrm{C}$, no pressure surge is predicted when the valve closes instantaneously, upon the detection of flow reversal. The pressure surge shows a large peak at the valve closure delay time of ca. $2 \mathrm{~s}$. Notably, further increase in valve delay time actually results in a reduction in the pressure head. This is primarily because the drop in the line pressure due to inventory loss has a more marked impact on reducing the pressure head that is developed during the latter stages of discharge. With reference to figure 4.1, in all other cases simulated, where ethylene is transported either in the supercritical $\left(60\right.$ and $\left.90 \mathrm{bar} ; 30{ }^{\circ} \mathrm{C}\right)$, liquid $\left(60 \mathrm{bar} ; 5^{\circ} \mathrm{C}\right)$ or vapour phase (30 and 45 bar), the pressure surge is relatively unaffected by the valve closure delay time if it is greater than ca. $4 \mathrm{~s}$. Also, comparison of figures $4.4(a)$ and $(b)$ shows that when transporting ethylene in vapour phase (30 and 45 bar), the initial fluid temperature has almost negligible impact on the resulting pressure surge.

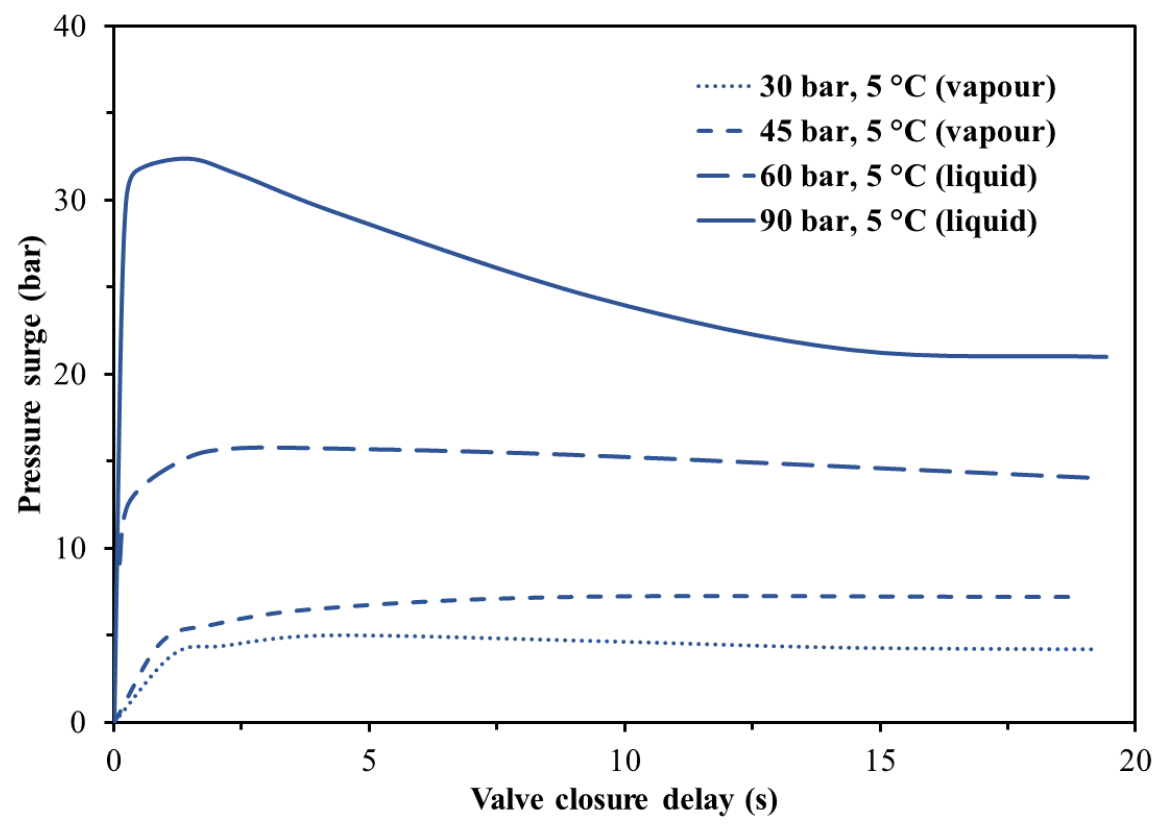

(a) 


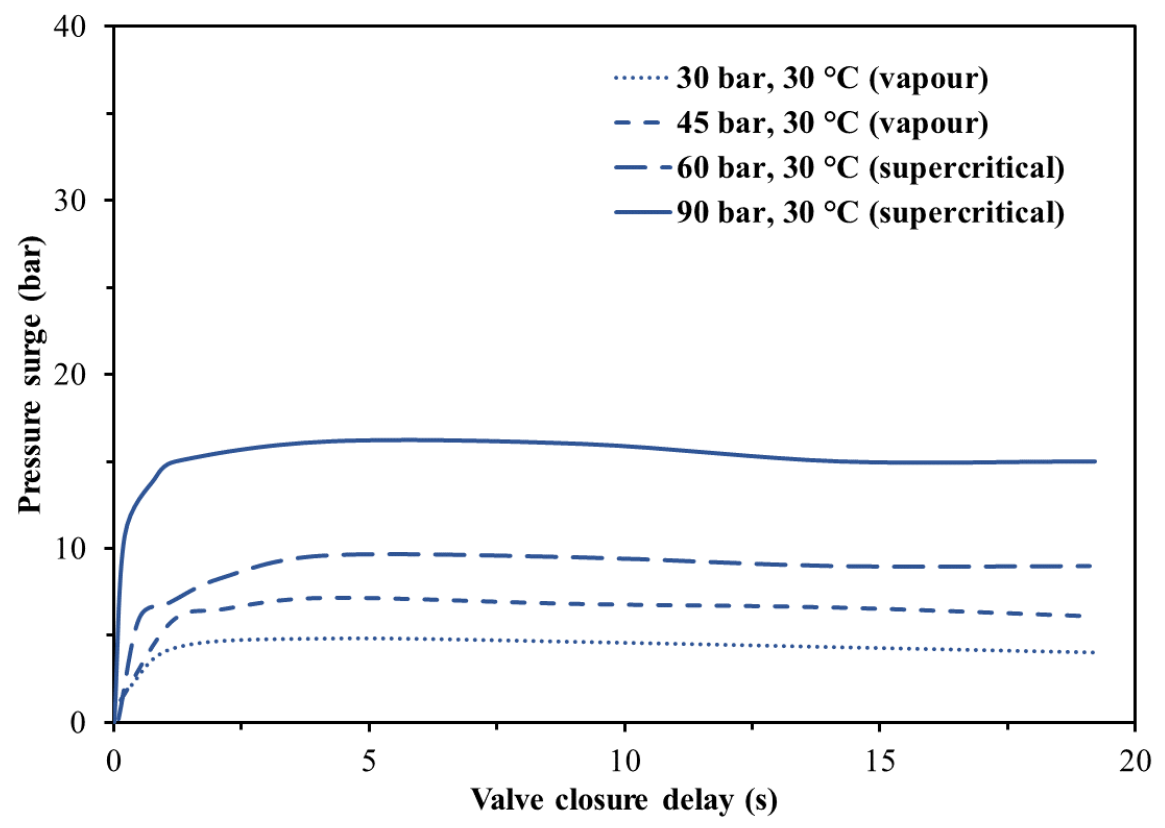

(b)

Figure 4.4: Variation of the pressure surge with the closure delay time, as predicted at the upstream side of a CV for $250 \mathrm{~mm}$ internal diameter pipeline transporting ethylene at various initial pressures and the initial temperatures of $5{ }^{\circ} \mathrm{C}(a)$ and $30{ }^{\circ} \mathrm{C}(b)$. The corresponding fluid phase for each case is stated.

Figure 4.5 presents the effect of ethylene transportation temperature on the magnitude of the pressure surge at pipeline initial pressures of 90, 60 and 45 bar for a $\mathrm{CV}$ with the closure delay time, $\Delta t_{d}=2 \mathrm{~s}$. 


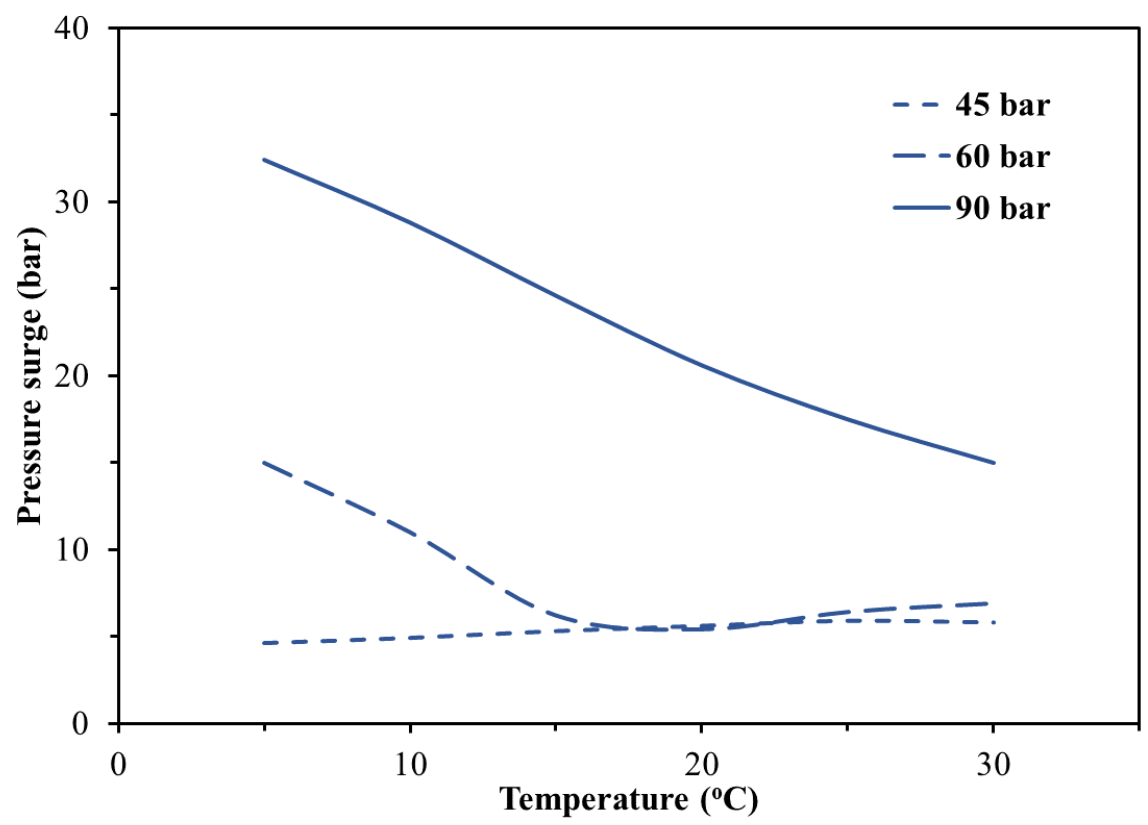

Figure 4.5: The effect of the ethylene transportation temperature on the pressure surge for different pipeline initial pressures on the upstream side of a CV with $\Delta t_{d}=2 \mathrm{~s}$.

As follows from equation 4.1, the fluid density and speed of sound are the two fluid properties affecting the pressure surge. In figure 4.6, the product $\rho a$ is plotted as a function of ethylene transportation temperature at different pipeline initial pressures corresponding to figure 4.5 . Comparison of the data in figures 4.5 and 4.6 shows that the pressure surge variations in figure 4.5 follow very similar trends to the variation of $\rho a$ in figure 4.6, explaining the observed decrease in pressure surge in figure 4.5 with the pipeline initial pressure at 90 and 60 bar, as well as the increase in the pressure surge with the pipeline initial pressure at 45 bar. 


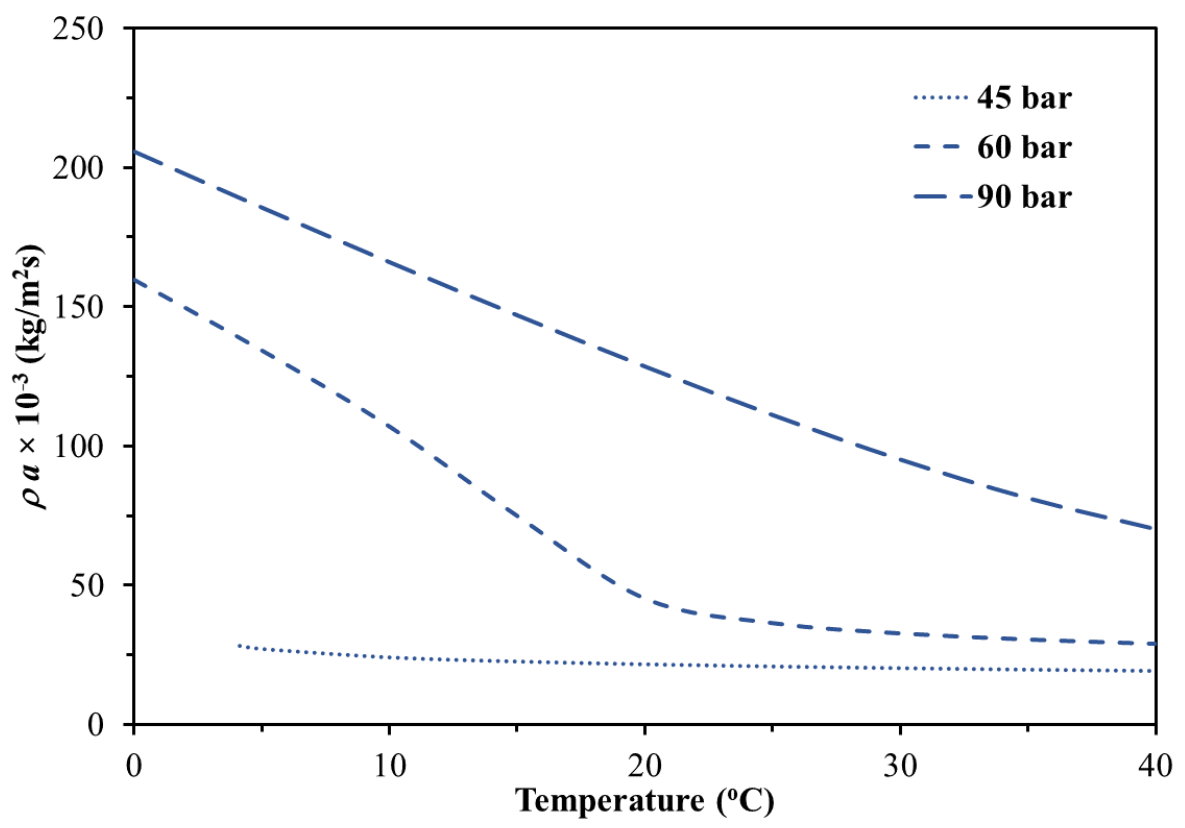

Figure 4.6: Variation of $\rho a$ with ethylene transportation temperature at 45, 60 and 90 bar pipeline initial pressures for ethylene, as predicted using the Peng- Robinson Equation of State (PR EoS).

In order to prevent pressure surges, the pipeline support/anchoring blocks are commonly designed based on an estimate of the thrust load, $F$ caused by the pressure surge passing across the pipe bends as given by (American Lifelines Alliance, 2001; Ord, 2006):

$$
F=\Delta p \cdot D L F \cdot A
$$

where, $\Delta p$ and $A$ are respectively the pressure surge and pipe cross-sectional area. $D L F$ is the dynamic magnification factor, which is set to a maximum value of 2 .

Using equation 4.2, the thrust load created by 5 to 32 bar pressure surges in a $250 \mathrm{~mm}$ i.d pipeline (figure 4.4) can be estimated to be in the range of between ca. 5 to 32 tonne. These forces are relatively large in comparison with the pipeline weight (ca. 7 tonne for one $100 \mathrm{~m}$ long section of $12 \mathrm{~mm}$ wall thickness $250 \mathrm{~mm}$ i.d. steel pipeline), indicating that surge protection measures should be carefully considered when using CVs in high-pressure ethylene pipelines. 


\subsection{The Study of ASV and RCV Activation Pressure}

\subsubsection{Simulation Test Setup}

In the following section, the impact of ASV and RCV activation pressure is assessed by determining the amount of total inventory loss prior to complete isolation following FBR and puncture failures. This draws upon the simulations of a $15 \mathrm{~km}$ length typical onshore ethylene pipeline specified in Section 4.2. Here, the initial line pressure and temperature are assumed to be 90 bar and $10{ }^{\circ} \mathrm{C}$ respectively. All other conditions are unchanged.

Figure 4.7 shows the schematic representation of the pipeline configuration assumed for simulation tests $1-6$. Two ESDVs spaced at a distance, $D$ are installed along a pipeline section, and the FBR/puncture located at a distance, $x$ downstream from the first valve (ESDV-1). The valve type, closure characteristics, including the corresponding failure scenarios for each test are presented in table 4.1 .

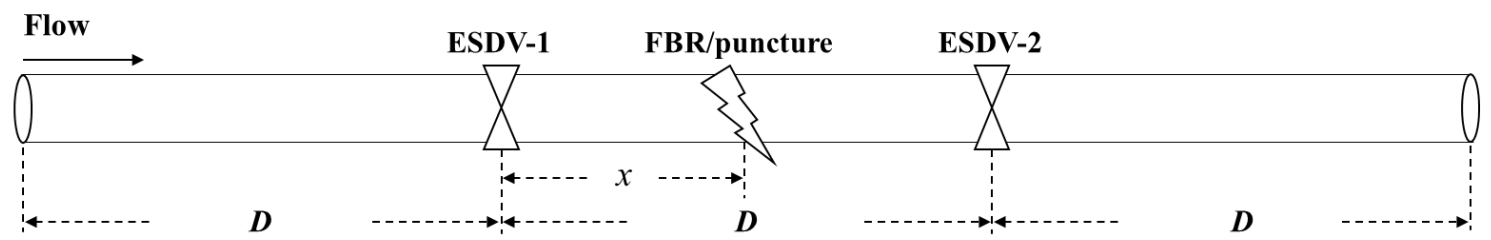

Figure 4.7: Schematic representation of the ethylene pipeline section indicating the valve configuration and the location of the valve relative to the failure point for tests $4.1-4.6$ (see table 4.1).

Table 4.1: Valve characteristics for tests $4.1-4.6$.

\begin{tabular}{|c|c|c|c|c|}
\hline \multirow{2}{*}{$\begin{array}{l}\text { Test } \\
\text { No. }\end{array}$} & \multirow{2}{*}{ Failure Type } & \multirow{2}{*}{$\begin{array}{c}\text { Failure } \\
\text { Location }(x / D)\end{array}$} & \multicolumn{2}{|c|}{ ESDV-1 \& ESDV-2 } \\
\hline & & & Valve type & Valve characteristics \\
\hline 4.1 & FBR & $\begin{array}{l}0.1,0.3,0.5 \\
\quad 0.7,0.9\end{array}$ & ASV & $\begin{array}{l}\text { valve linear closure rate }= \\
\begin{array}{l}2.54 \mathrm{~cm} / \mathrm{s}, \Delta p_{a}= \\
2 / 5 / 10 / 20 / 30 \mathrm{bar}\end{array}\end{array}$ \\
\hline
\end{tabular}




\begin{tabular}{|c|c|c|c|c|}
\hline 4.2 & Puncture (125 mm) & $\begin{array}{l}0.1,0.3,0.5 \\
\quad 0.7,0.9\end{array}$ & ASV & $\begin{array}{l}\text { valve linear closure rate }= \\
\begin{array}{l}2.54 \mathrm{~cm} / \mathrm{s}, \Delta p_{a}= \\
2 / 5 / 10 / 20 / 30 \text { bar }\end{array}\end{array}$ \\
\hline 4.3 & Puncture (10 mm) & $\begin{array}{l}0.1,0.3,0.5 \\
\quad 0.7,0.9\end{array}$ & ASV & $\begin{array}{l}\text { valve linear closure rate }= \\
2.54 \mathrm{~cm} / \mathrm{s}, \Delta p_{a}= \\
2 / 5 / 10 / 20 / 30 \text { bar }\end{array}$ \\
\hline 4.4 & FBR & $\begin{array}{c}0.1,0.3,0.5 \\
0.7,0.9\end{array}$ & $\mathrm{RCV}$ & $\begin{array}{c}\text { valve linear closure rate }= \\
2.54 \mathrm{~cm} / \mathrm{s}, \Delta p_{a}= \\
2 / 5 / 10 / 20 / 30 \mathrm{bar}, t_{a} \\
(\mathrm{RCV})=t_{a}(\mathrm{ASV})+60 \mathrm{~s}\end{array}$ \\
\hline 4.5 & Puncture (125 mm) & $\begin{array}{c}0.1,0.3,0.5 \\
0.7,0.9\end{array}$ & $\mathrm{RCV}$ & $\begin{array}{c}\text { valve linear closure rate }= \\
2.54 \mathrm{~cm} / \mathrm{s}, \Delta p_{a}= \\
2 / 5 / 10 / 20 / 30 \mathrm{bar}, t_{a} \\
(\mathrm{RCV})=t_{a}(\mathrm{ASV})+60 \mathrm{~s}\end{array}$ \\
\hline 4.6 & Puncture (10 mm) & $\begin{array}{l}0.1,0.3,0.5 \\
\quad 0.7,0.9\end{array}$ & $\mathrm{RCV}$ & $\begin{array}{c}\text { valve linear closure rate }= \\
2.54 \mathrm{~cm} / \mathrm{s}, \Delta p_{a}= \\
2 / 5 / 10 / 20 / 30 \mathrm{bar}, t_{a} \\
(\mathrm{RCV})=t_{a}(\mathrm{ASV})+60 \mathrm{~s}\end{array}$ \\
\hline
\end{tabular}

The closure for both ASVs and RCVs are assumed to occur during the time frame between the valve activation time, $t_{a}$ and its complete closure time, $t_{c}$. In the case of ASVs, the valve activation time, $t_{a}(\mathrm{ASV})$ corresponds to the time when the line pressure drops below a pre-set pressure. The valve activation pressure, $\Delta p_{a}$ is defined as a certain threshold below the nominal operating pressure. Hence, $t_{a}$ (ASV) depends on the fluid decompression wave velocity and the distance between the pipe failure location and the valve. RCV activation time, $t_{a}(\mathrm{RCV})$ on the other hand is defined as the sum of the time lapse for detection of the pressure drop at the valve location (here equals to $t_{a}(\mathrm{ASV})$ ) and the operator response/action time to activate valve closure. In this study, the operator response/action time is assumed to be $60 \mathrm{~s}$. It should be noted that for both ASVs and RCVs, the valve linear closure rate is assumed to be $2.54 \mathrm{~cm} / \mathrm{s}$ ( $1 \mathrm{in} / \mathrm{s}$ ), which is the standard rate used in practice for such diameter pipelines (Smith and Zappe, 2004).

Returning to table 4.1, for tests 4.1 to 4.3, ASVs are denoted as ESDV-1 and ESDV-2, with activation pressure, $\Delta p_{a}$ varying from 2 bar to 30 bar (1/3 of pipeline initial pressure). Tests $4.1-4.3$ respectively simulate a FBR, a large puncture $(125 \mathrm{~mm})$ and a small puncture (10 $\mathrm{mm}$ ). For tests $4.4-4.6$, the valve type is replaced with RCV, with other parameters kept the 
same as tests $4.1-4.3$. In all tests, 5 failure locations are simulated corresponding to $x / D=0.1$, $0.3,0.5,0.7$ and 0.9 .

The total inventory loss, $M_{\text {total }}$ from a ruptured pipeline is given by:

$$
M_{\text {total }}=\int_{0}^{t_{c}} \dot{m} d t+A \int_{0}^{D} \rho\left(x, t_{c}\right) d x
$$

The first term on the right-hand side of equation 4.3 represents the cumulative mass released from the pipeline prior to complete valve closure. This is evaluated as an integral of the discharge flow rate, $\dot{m}$, predicted from the flow model developed in Chapter 2. The second term on the right-hand side corresponds to the amount of inventory remaining in the isolated section of the pipe at the moment of complete closure of both valves. The remaining symbols have been defined previously.

The total inventory loss calculated using equation 4.3 is compared with the mass of fluid present in the isolated pipeline section (i.e. the initial mass of inventory), $M_{o}$ prior to failure as follows:

$$
M_{o}=\rho_{o} \cdot A \cdot D
$$

Here, $\rho_{o}$ is the fluid density at the prevailing pipeline transportation pressure and temperature.

In order to investigate the impact of the increasing activation pressure, $\Delta p_{a}$ on different valve types, the comparison among the total inventory loss, $M_{\text {total }}$ is needed. However, given that at each $\Delta p_{a}$, five failure locations are simulated (table 4.1), seeking a comparison would be difficult. Hence, the average of the total inventory loss for all 5 failure locations tested at a given $\Delta p_{a}, \bar{M}_{\text {total }}$ is introduced for easier comparison:

$$
\bar{M}_{\text {total }}=\frac{\sum_{i=1}^{n} M_{t o t a l}(i)}{n}
$$


where, $n$ is number of failure locations tested at a given $\Delta p_{a} . i$, is the index of summation lying in the interval $[1, n] . M_{\text {total }}(i)$ on the other hand, is the total inventory loss at $i$ as calculated from equation 4.3 .

\subsubsection{Results and Discussion}

Figure 4.8 shows the predicted variation of the ASV activation time, $t_{a}(\mathrm{ASV})$ against valve activation pressure, $\Delta p_{a}$ for test 4.1 (see table 4.1) for a valve spacing distance, $D$ of $5 \mathrm{~km}$.

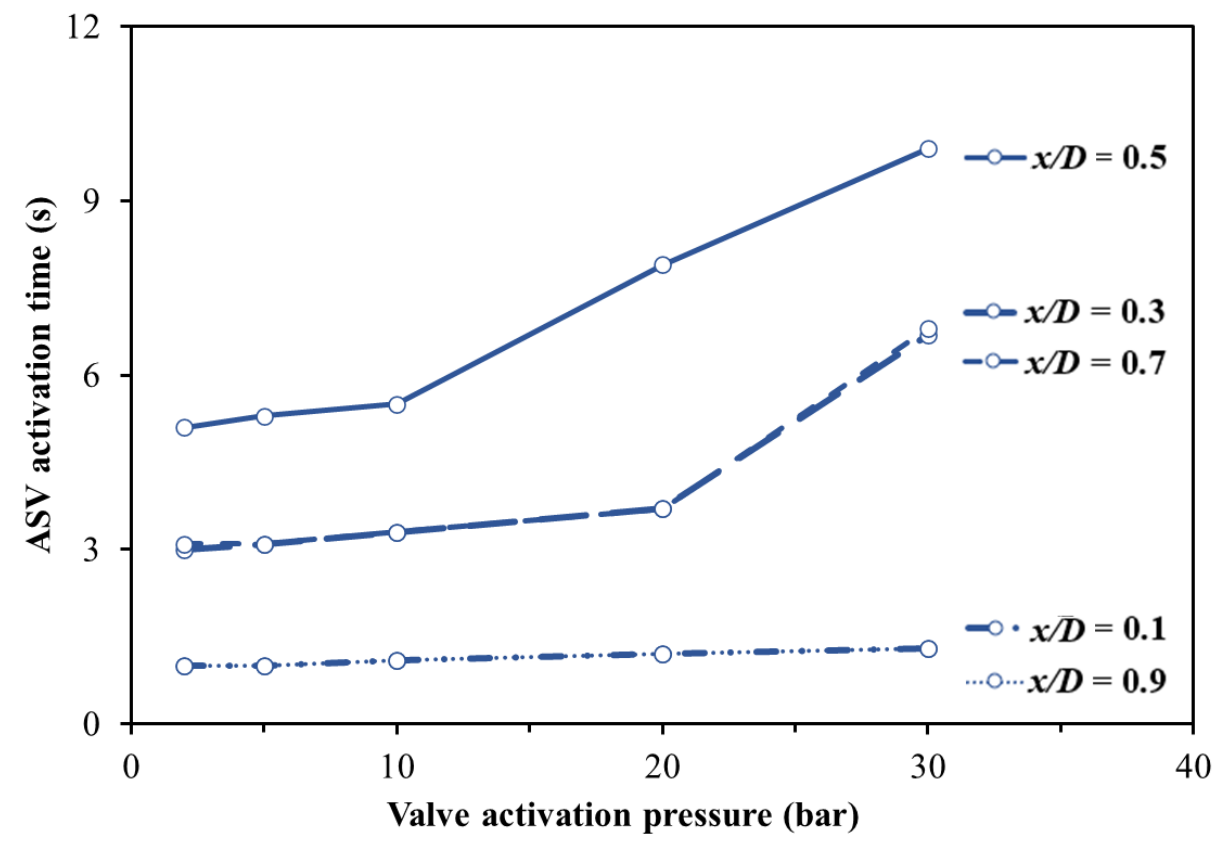

Figure 4.8: Test 4.1 variation of ASV activation time with valve activation pressure for different FBR locations along the pipeline.

As can be observed from figure 4.8, $t_{a}(\mathrm{ASV})$ remains relatively constant for FBR located close to either of the valves $(x / D=0.1$ and 0.9$)$ whilst $\Delta p_{a}$ increases from 2 bar (ca. 1 s) to 30 bar (ca. $1.2 \mathrm{~s}$ ). Turning to another pair; $x / D=0.3$ and $0.7, t_{a}(\mathrm{ASV})$ is relatively constant albeit marginally increasing from ca. $3.1 \mathrm{~s}\left(\Delta p_{a}=2\right.$ bar) to $3.7 \mathrm{~s}\left(\Delta p_{a}=20\right.$ bar). This is followed by a rapid rise to ca. $6.8 \mathrm{~s} \mathrm{(} \Delta p_{a}=30$ bar). As for $x / D=0.5$, a gradual increase can be observed for $t_{a}$ (ASV), with $\Delta p_{a}$ from 2 to 10 bar. The subsequent rate of increase increases and reaches ca. $10 \mathrm{~s}$ at $\Delta p_{a}=30$ bar. Remarkably, the increase of $\Delta p_{a}$ in test 4.1 does not have a significant impact on $t_{a}(\mathrm{ASV})$ and relatively fast ASV activation times can be noted for all FBR locations 
(e.g. maximum $10 \mathrm{~s}$ for $x / D=0.5 ; 6.8 \mathrm{~s}$ for $x / D=0.3$ and $0.7 ; 1.2 \mathrm{~s}$ for $x / D=0.1$ and 0.9 ). This may be explained by the onset of the FBR resulting in choked flow occurring at the release point, which is marked by an initial rapid drop in the release pressure (i.e. more than 50 bar). Also, the initial velocity of the flow in the pipe $(1 \mathrm{~m} / \mathrm{s})$ is relatively small compared to the speed of the expansion wave propagation in the pipe (ca. $90 \mathrm{~m} / \mathrm{s}$ ) upon FBR. As such, $t_{a}$ (ASV) is mainly affected by the distance between the valve and the rupture plane, and not the initial inpipe flow.

Figure 4.9 shows the variation of the inventory loss ratio versus $\Delta p_{a}$ for test 4.1 . Where the inventory loss ratio $=$ the average of the total inventory loss, $\bar{M}_{\text {total }} /$ the initial mass of inventory, $M_{o}$ (calculated by equation 4.4). As can be observed, the inventory loss ratio increases slightly when $\Delta p_{a}$ increases from 2 to 30 bar. It is worth noting that the inventory loss ratio at any $\Delta p_{a}$ is less than ca. $105 \%$. This again indicates that in test 4.1, as expected, short ASV activation times enable rapid isolation of the pipeline segment.

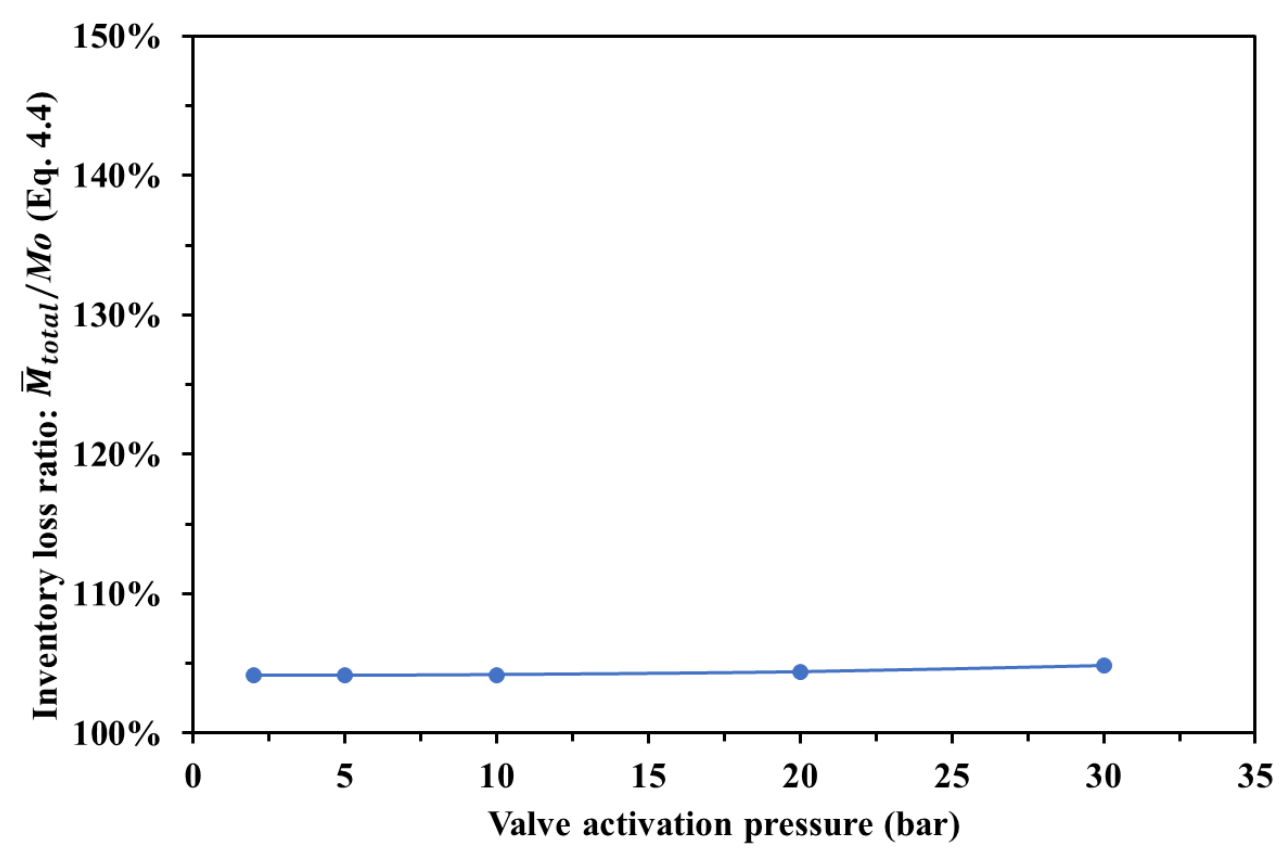

Figure 4.9: Test 4.1 variation of the inventory loss ratio with valve activation pressure.

Figure 4.10 shows the variation of the ASV activation time, $t_{a}$ (ASV) as a function of the valve activation pressure, $\Delta p_{a}$ for test 4.2 (table 4.1 ) at $5 \mathrm{~km}$ valve spacing for a relatively large puncture (125 mm (half of pipeline i.d.)) between the valves. Similar behaviours in the trends as compared to figure 4.8 can be observed, with a small rate of increase in $t_{a}$ (ASV) for all 
puncture locations between, $\Delta p_{a}=2$ and 10 bar, followed by a more rapid increase, reaching up to $\Delta p_{a}=30$ bar (e.g. approximately $5.8 \mathrm{~s}, 15.1 \mathrm{~s}, 10.7 \mathrm{~s}, 7.7 \mathrm{~s}$ and $5 \mathrm{~s}$ for $x / D=0.1,0.3,0.5$, 0.7 and 0.9 , respectively). In comparison to test $4.1, t_{a}$ (ASV) in test 4.2 is higher between, $\Delta p_{a}=10$ to 30 bar, but still relatively fast ASV activations can be seen for all these puncture locations. This is due to the initial velocity of the flow in the pipe $(1 \mathrm{~m} / \mathrm{s})$ being relatively small compared to the speed of the expansion wave propagation (ca. $65 \mathrm{~m} / \mathrm{s})$ in the pipe for a large puncture.

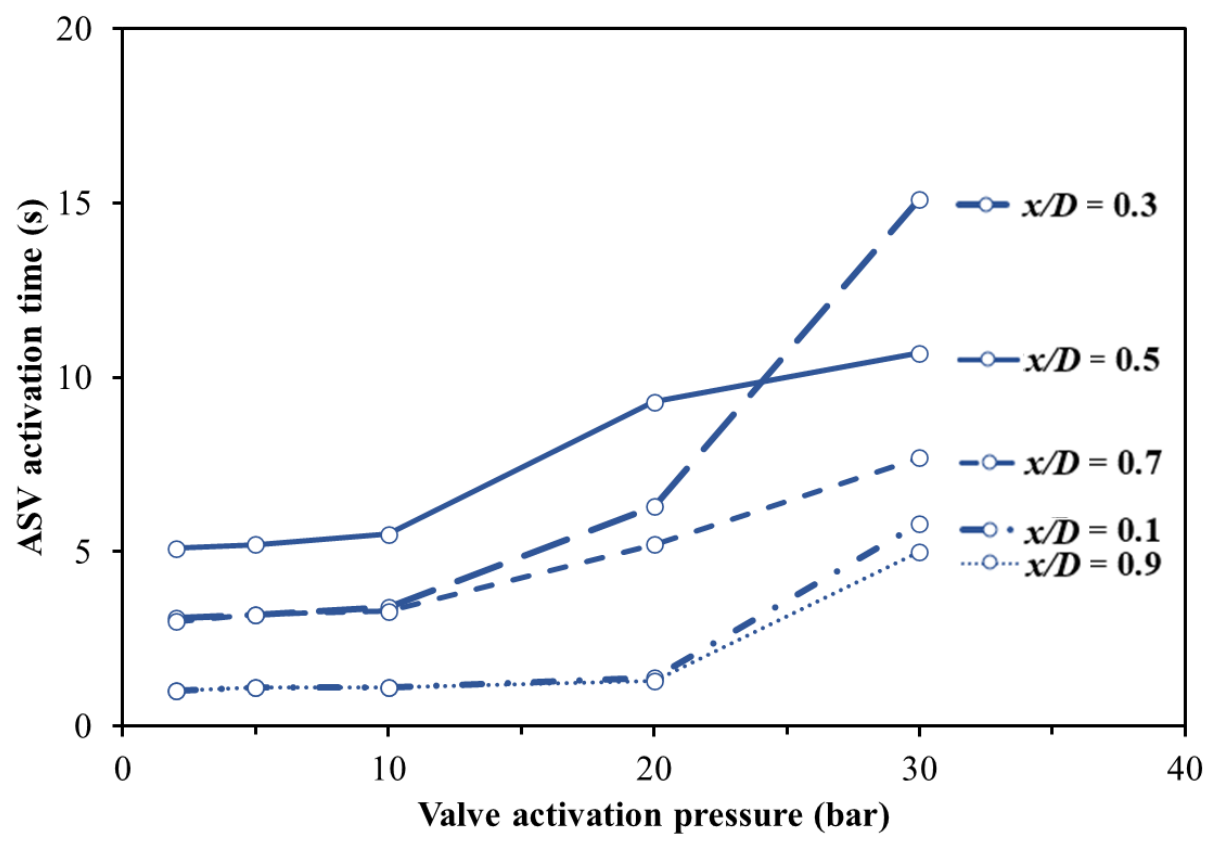

Figure 4.10: Test 4.2 variation of ASV activation time with valve activation pressure for different puncture (125 $\mathrm{mm}$ dia.) locations along the pipeline.

Figure 4.11 shows the variation of the inventory loss ratio versus the valve activation pressure for test 4.2. Similar trends as compared to figure 4.9 can be observed in figure 4.11 as rationalised above. 


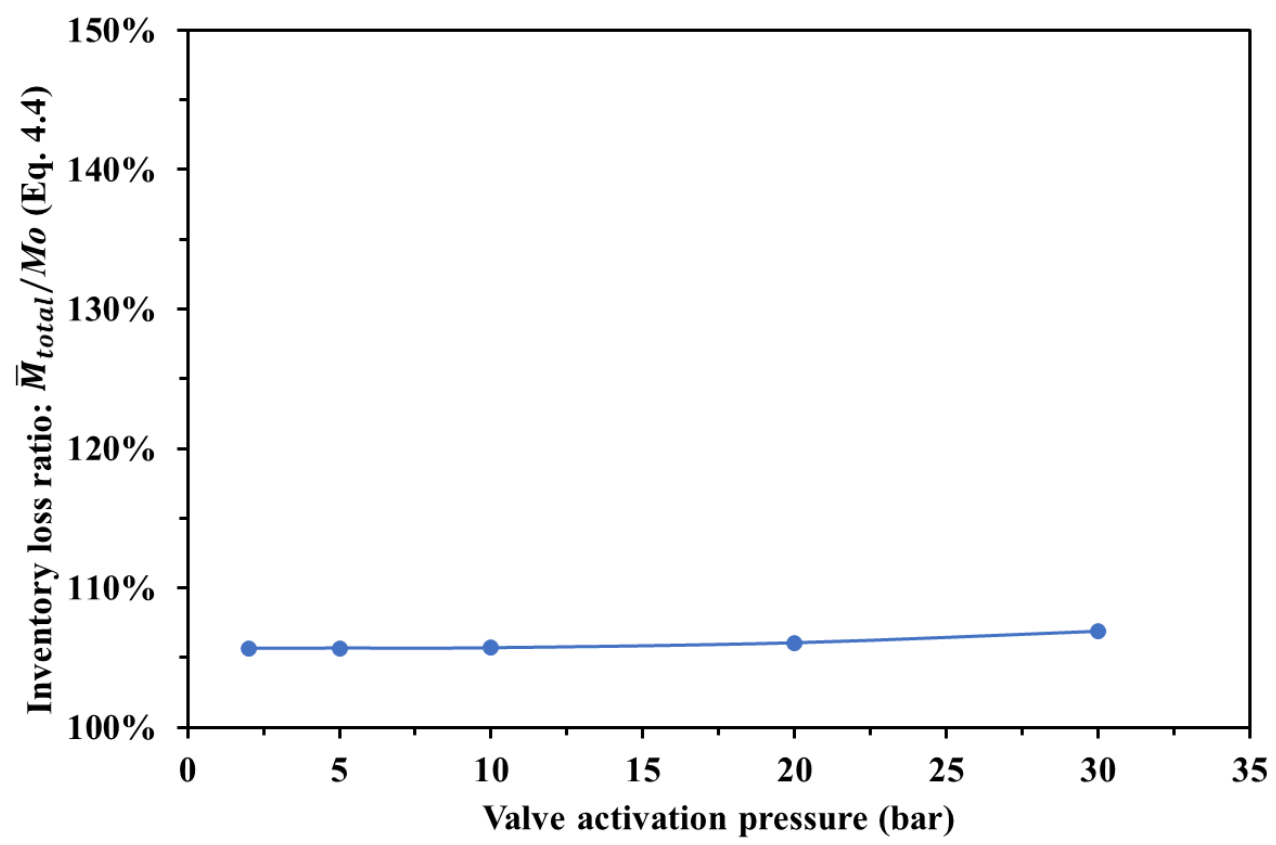

Figure 4.11: Test 4.2 variation of the inventory loss ratio with valve activation pressure.

Turning to test 4.3 (table 4.1), figure 4.12 shows the variation of pressure at both the puncture and ASV-1 locations with time when $x / D=0.5$. Upon failure, choked flow occurs at the release point (puncture location, solid line), which is marked by an initial rapid drop in the release pressure from 90 bar to ca. 36 bar. As the decompression proceeds, the release pressure remains almost constant. This can be explained by the infinite reservoir assumed at the upstream boundary conditions where feed conditions are unaffected by the decompression process. In terms of the pressure at ASV-1 location (dotted line), as the expansion wave propagating, it undergoes a continuous drop at ca. $2 \mathrm{~s}$ and remains almost constant after ca. $12 \mathrm{~s}$. It can particularly be noted that due to the infinite reservoir upstream boundary condition and the small puncture size, total pressure drop at ASV - 1 location during decompression is less than ca. 2.5 bar. Hence ASVs will not be activated when the activation pressure is more than 2.5 bar.

Figure 4.13 shows the variation of inventory loss ratio and the ASV activation time, $t_{a}$ (ASV) as a function of puncture location between two ASVs at $\Delta p_{a}=2$ bar. It should be noted that, $t_{a}$ (ASV) for test 4.3 at $\Delta p_{a}=2$ bar is significantly longer compared to tests 4.1 and 4.2 (figures 4.8 and 4.10, respectively). As a result, the mass loss prior to complete isolation for test 4.3 at $\Delta p_{a}=2$ bar is considerably larger compared to those for tests 4.1 (figure 4.9) and 4.2 (figure 4.11). 


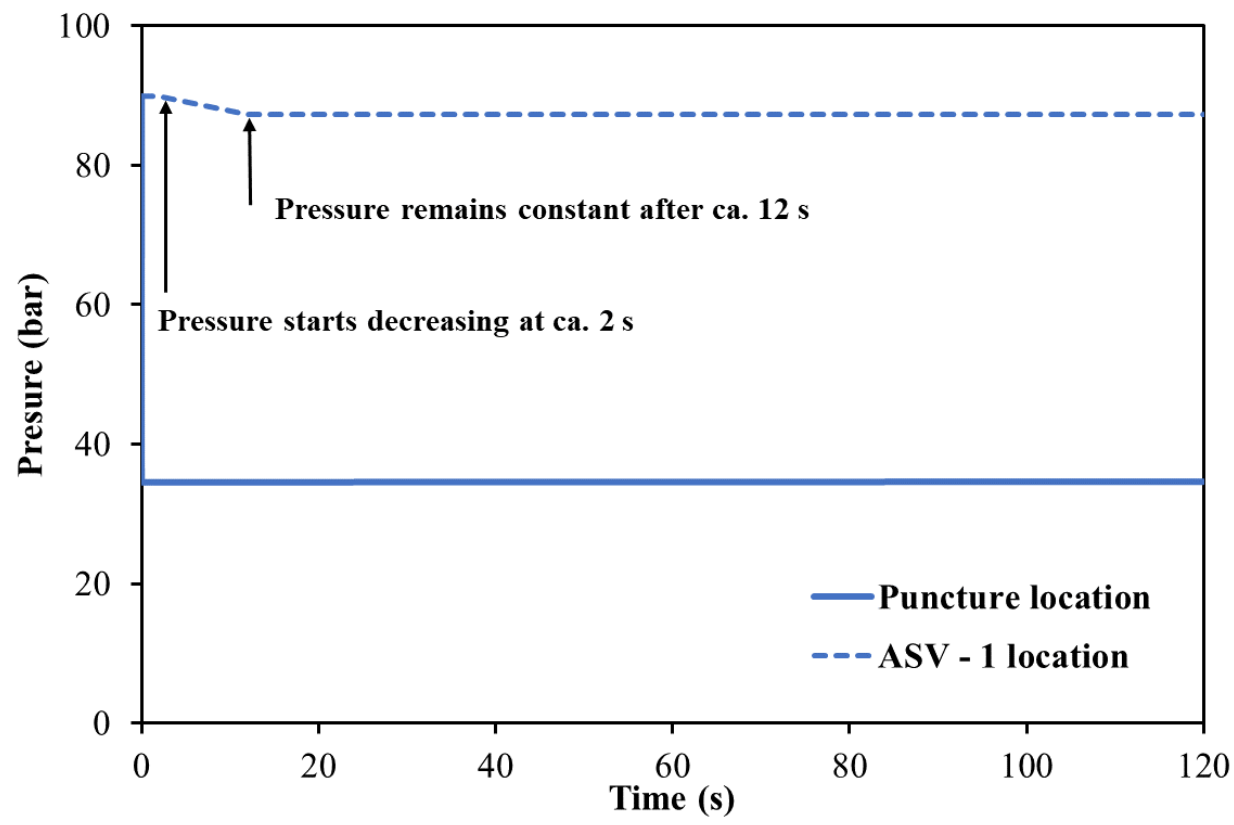

Figure 4.12: Test 4.3 variation of puncture location pressure and ASV-1 location pressure with time $(x / D=0.5)$.

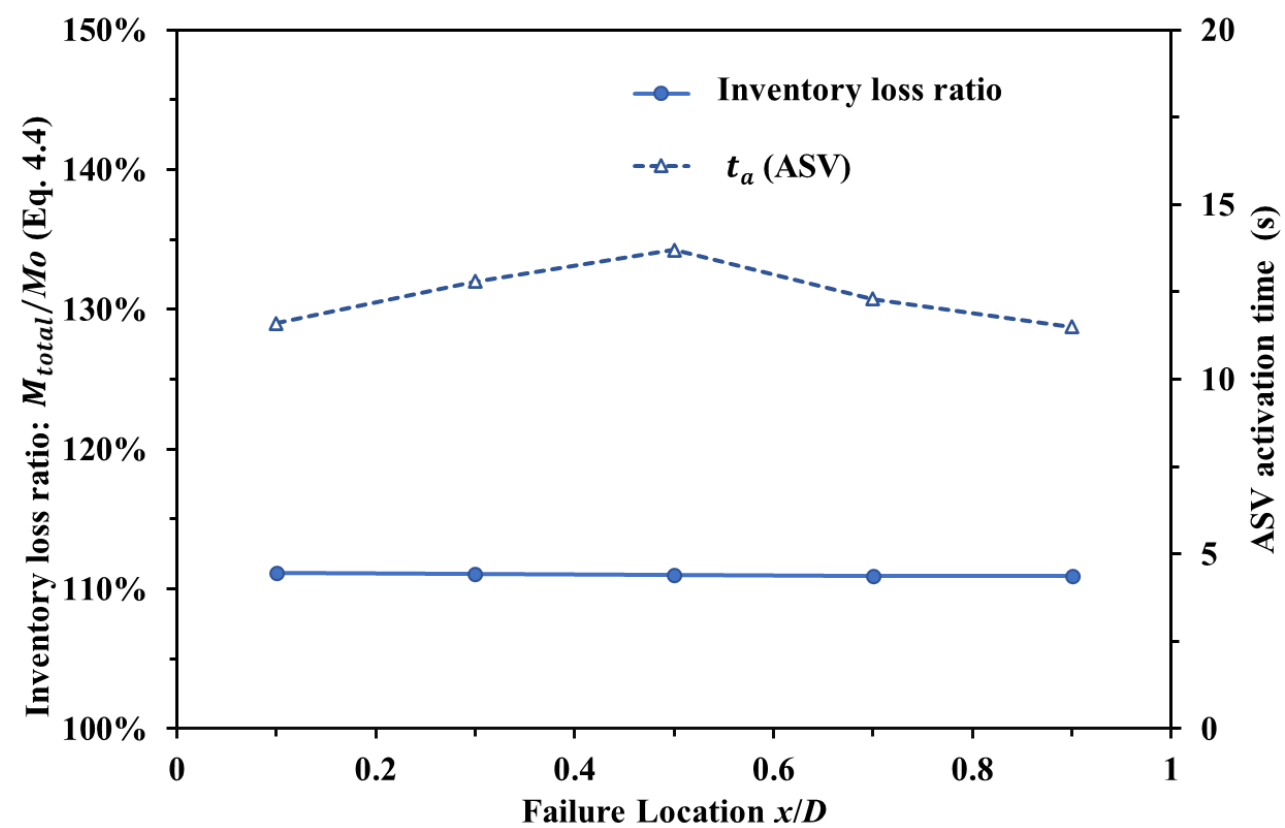

Figure 4.13: Test 4.3 variation of the inventory loss ratio (left side) and ASV activation time (right side) as a function of puncture location between two ASVs at $\Delta p_{a}=2$ bar.

Figure 4.14 shows the variation of the inventory loss ratio versus valve activation pressure, $\Delta p_{a}$ for all 6 tests presented in table 4.1. For tests $4.4-4.6$, the valve type employed is RCV, with all the remaining parameters being the same as for tests $4.1-4.3$. As expected, ASVs 
(tests 4.1 - 4.3) offer a significant reduction in the inventory mass loss prior to complete valve closure as compared to RCVs (tests $4.4-4.6$ ). This is due to the additional operator action time (60s) required for the activation of RCVs, during which large amount of fluid will still escape from the pipeline. Remarkably, the largest reduction in the inventory mass loss by using ASVs is observed in the case of FBR (tests 4.1 and 4.4). However, such reduction decreases in the case of a large puncture ( $125 \mathrm{~mm}$, tests 4.2 and 4.5$)$ and becomes negligible for a small puncture (10 mm, tests 4.3 and 4.6). This means that using ASVs becomes less favourable as puncture size decreases.

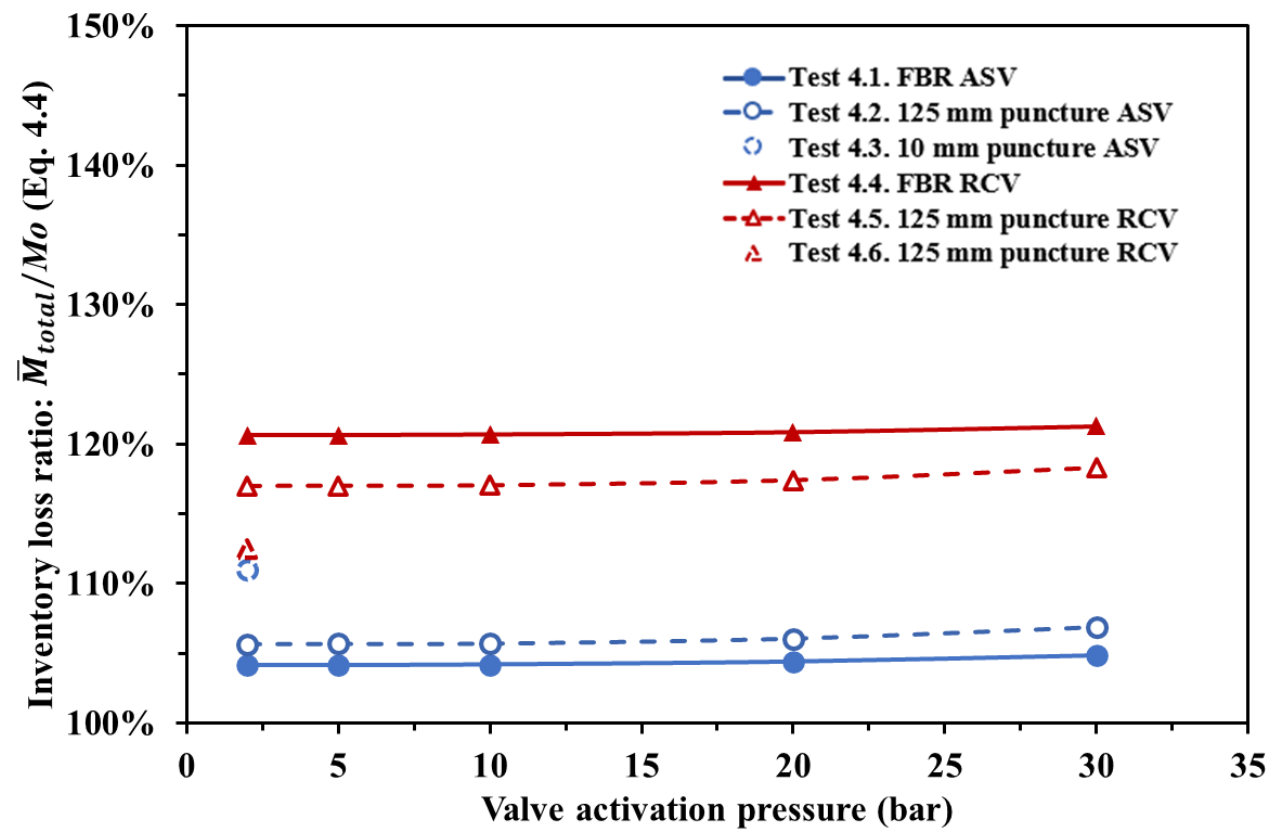

Figure 4.14: Variation of the inventory loss ratio with valve activation pressure for tests 4.1 -4.6 .

\subsection{Concluding Remarks}

Using the HEM model presented in Chapter 2, this chapter focused on modelling followed by comparing and contrasting the dynamic response of different types of ESDVs following the accidental failure of high-pressure pipelines. An ethylene pipeline was used as a case study given the chemical's extensive pipeline transportation across the globe and its hazardous nature as being both flammable, explosive and potentially toxic at high concentrations. Furthermore, 
its rapid expansion following a pipeline failure may result in temperatures as low as $-100{ }^{\circ} \mathrm{C}$ giving rise to the serious risk of a catastrophic brittle running facture.

The investigations conducted in this chapter are of paramount importance in practice as the results can assist pipeline operators to select the appropriate type of ESDV for a given scenario, taking into account the ESDV characteristics such as its activation and closure times, any resulting pressure surges and ultimately, the amount of inventory lost prior to complete pipeline isolation. The latter information is particularly important as it serves as the source term for determining the consequences of pipeline failure such as fires, explosions, toxic releases and environmental pollution. These ultimately dictate the minimum pipeline safe distances to populated areas and emergency response planning.

Based on the results and analysis presented, the following key conclusions of practical significance may be made:

- The investigation of pressure surges upon CV closure for a wide range of ethylene pipeline operating pressures and temperatures was carried out. It was found that the pressure surge magnitude generally decreased with the operating pressure and temperature, varying non-linearly with the valve closure delay time.

- It is noteworthy that for the ethylene pipeline test case operating at a pressure of $90 \mathrm{bar}$, the maximum amplitude of pressure surges was found to be ca. 32 bar. This could lead to a large thrust or bending forces acting on the pipeline segments, potentially damaging the pipeline.

- The impact of ASV and RCV activation pressure was assessed by determining the amount of total inventory loss (i.e. the sum of the mass released from the pipeline prior to complete valve closure and the amount of inventory remaining in the isolated section of the pipe at the moment of complete valve closure) prior to complete isolation following pipeline FBR and different generic sizes of puncture failures. In the case of failure locations along the pipe, it was found that for failures located close to either of the valves (i.e. $x / D=0.1$ and 0.9 ), the ASV activation time remained relatively constant with increasing valve activation pressure. However, when the failures located in the middle between two valves (i.e. $x / D=0.5$ ), the ASV activation time grew significantly with the increasing valve activation pressure. Such results indicated that the ASV 
activation time was mainly affected by the distance between the valve and the rupture plane, but not the initial in-pipe flow.

- In the case of FBR and large puncture (i.e. $\geq 50 \%$ of pipe i.d.) failures, decreasing valve activation pressure resulted in a reduction in the total inventory loss prior to complete valve closure. However, the reduction in the total inventory loss did not change significantly with a decrease in the valve activation pressure. Turning to a small puncture (i.e. $10 \mathrm{~mm}$ ), for the cases studied, both ASVs and RCVs would not activate with a large valve activation pressure (i.e. more than 2.5 bar) due to the fact that the pressure drop at the valve location was found to be relatively small throughout the decompression.

- Comparisons of using either ASVs or RCVs along the pipeline showed that ASVs offered significant reduction in the total inventory loss prior to complete isolation as compared to RCVs. This was due to the additional operator action time required for the activation of RCVs, during which large amount of fluid would still escape from the pipeline. However, using ASVs became less favourable as puncture size decreased. In such circumstances, the capital and operating costs of either of the valves will also be important factors governing their selection.

In the next chapter, the efficacy of ESDVs by using different inline valve combinations and spacing in limiting and ultimately isolating outflow following the accidental failure of highpressure pipelines is investigated. 


\section{Chapter 5:}

\section{The Efficacy of Emergency Shutdown Valve following the Accidental Failure of High-pressure Pipelines}

\subsection{Introduction}

As discussed in Chapter 1, the ensuing in-pipe fluid flow dynamics following pipeline failure, and hence the outflow is dependent on the ESDV type employed. In addition, valve capital costs vary significantly depending on their type (Smith and Zappe, 2004). Therefore, the efficacy of ESDV as well as the number of ESDVs installed and their spacing along pipelines, must be optimised in order to achieve a balance between valve capital cost and risk reduction. Also, more specifically, given that CVs are designed to prevent backflow, they cannot be considered as the only resort for emergency isolation in long multi-segment pipelines, but should be setup together with ASVs or RCVs. This calls for characterisation of the efficacy of CVs as an auxiliary type of ESDV in long pipelines.

In this chapter, adopting the flow model incorporating the valve closure boundary conditions described in Chapter 2, the efficacy of ESDV following the accidental failure of high-pressure pipelines is investigated. Ethylene and $\mathrm{CO}_{2}$ are chosen as the case examples of the fluids being transported. As mentioned in Chapter 4, ethylene is chosen given the chemical's extensive pipeline transportation across the globe and its hazardous nature as being both flammable, explosive and potentially toxic at high concentrations. $\mathrm{CO}_{2}$ on the other hand is selected given its hazardous nature (an asphyxiant at $>7 \% \mathrm{v} / \mathrm{v}$ ) and the enormous amounts of $\mathrm{CO}_{2}$ destined to be transported using high-pressure pipelines as part of the Carbon Capture and Storage (CCS) chain across the globe. As stated in Chapter 1, given CCS' significant potential in mitigating global warming, the worldwide $\mathrm{CO}_{2}$ pipeline network length is estimated to exceed at 100,000 km by 2030 (International Energy Agency, 2009). As such, the quantitative failure consequence assessment of high-pressure $\mathrm{CO}_{2}$ transmission pipelines is essential.

For the sake of completeness, two types of pipeline failures, including Full Bore Rupture (FBR) and puncture are considered in this chapter. FBR is assumed to occur along an ethylene pipeline, as being the most catastrophic type of pipeline failure possible in the offshore industry. On the other hand, for the $\mathrm{CO}_{2}$ pipeline, puncture failure is assumed. Although in 
comparison to pipeline FBR failure, pipeline puncture failure is much less catastrophic, its significantly higher failure frequency (Goodfellow et al., 2019) requires equal attention when performing quantitative failure consequence assessment for high-pressure transmission pipelines.

This chapter is organised as follows.

Section 5.2 presents the fluid flow simulations upon valve closure for a typical onshore $\mathrm{CO}_{2}$ pipeline following a puncture failure. Different valve spacing of RCVs and CVs are tested, and the results are presented and discussed in terms of the release pressure and temperature, the discharge mass flowrate and the cumulative mass discharged, as a function of time during decompression.

In Section 5.3, the efficacies of CVs, RCVs and ASVs and their combinations for the emergency isolation of a high-pressure ethylene pipeline following FBR are presented and discussed based on the comparison of the amount of total inventory loss prior to complete valve closure. Conclusions are drawn in Section 5.4.

\subsection{The Study of CV and RCV Spacing}

\subsubsection{Simulation Test Setup}

In this section, the simulations presented are based on the decompression of a hypothetical 5 $\mathrm{km}$ length, $200 \mathrm{~mm}$ i.d. thermally insulated (heat transfer coefficient $=5 \mathrm{~kW} / \mathrm{m}^{2} \mathrm{~K}$ ) pipeline filled with dense phase $\mathrm{CO}_{2}$ initially at 150 bar and $293.15 \mathrm{~K}$. A $100 \mathrm{~mm}$ i.d. puncture is assumed to occur mid-way along the length of the pipe. All relevant details regarding the pipeline setup are summarised in table 5.1. With the exception of a shorter length in order to keep the computational workload to a manageable level, the remaining selected conditions are representative of a typical onshore $\mathrm{CO}_{2}$ pipeline (Patchigolla and Oakey, 2013).

A total of 15 tests using different valve configurations are split into 3 scenarios. In scenario 1 (tests 5.1 to 5.5), only CVs are installed, and the number of equally spaced valves vary from 0 to 8 . For the second scenario (tests 5.6 to 5.10), the valve type is switched to RCV, with other details kept the same as the previous scenario. The third scenario (tests 5.11 to 5.15) concerns 
using both CVs and RCVs at the same locations (i.e. in tandem). The corresponding layout of valve configurations for scenarios 1 to 3 are depicted in figures 5.1 to 5.3 respectively.

Table 5.1: Input conditions for tests 5.1 to 5.15 .

\begin{tabular}{|c|c|c|}
\hline \multicolumn{2}{|l|}{ Input } & \multirow{2}{*}{$\begin{array}{l}\text { Value } \\
200\end{array}$} \\
\hline Pipeline & Pipeline outer diameter $(\mathrm{mm})$ & \\
\hline & Pipeline wall thickness (mm) & 10 \\
\hline & Pipeline roughness height $(\mathrm{mm})$ & 0.005 \\
\hline & Pipeline length (km) & 5 \\
\hline & Pipeline inclination $\left(^{\circ}\right)$ & 0 \\
\hline \multirow[t]{3}{*}{ Type of failure } & $\begin{array}{l}\text { Position of failure from high } \\
\text { pressure end }(\mathrm{km})\end{array}$ & 2.5 \\
\hline & Type of failure & Puncture in the middle \\
\hline & Orifice size $(\mathrm{mm})$ & 100 \\
\hline \multirow[t]{2}{*}{ Boundary conditions } & Upstream boundary condition & Constant pressure (Reservoir) \\
\hline & Downstream boundary condition & Outflow (Open end) \\
\hline \multirow[t]{5}{*}{ Initial conditions } & Pressure in pipeline (bar) & 150 \\
\hline & Temperature in pipeline $(\mathrm{K})$ & 293 \\
\hline & Ambient pressure (bara): & 1 \\
\hline & Ambient temperature $(\mathrm{K})$ : & 298.15 \\
\hline & Feed flow rate $\left(\mathrm{kgs}^{-1}\right)$ & 5 \\
\hline \multirow[t]{2}{*}{ RCV parameters } & Valve linear closure rate $\left(\mathrm{cms}^{-1}\right)$ & 1 \\
\hline & Valve activation time (s) & 120 \\
\hline
\end{tabular}




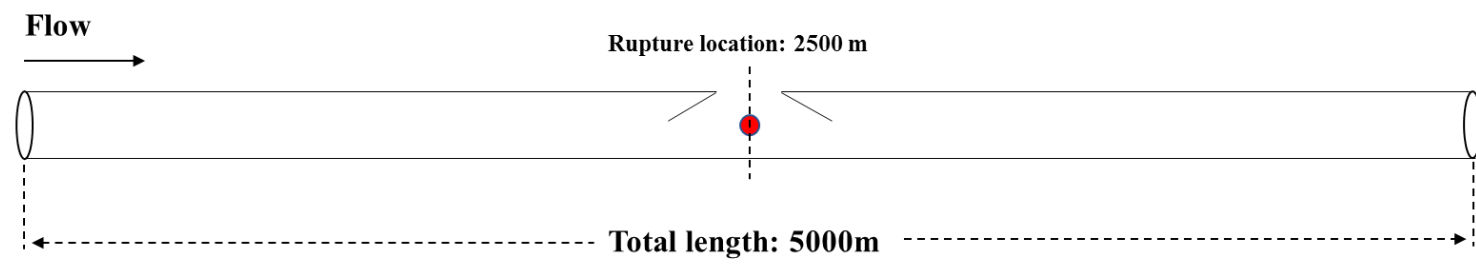

(a)

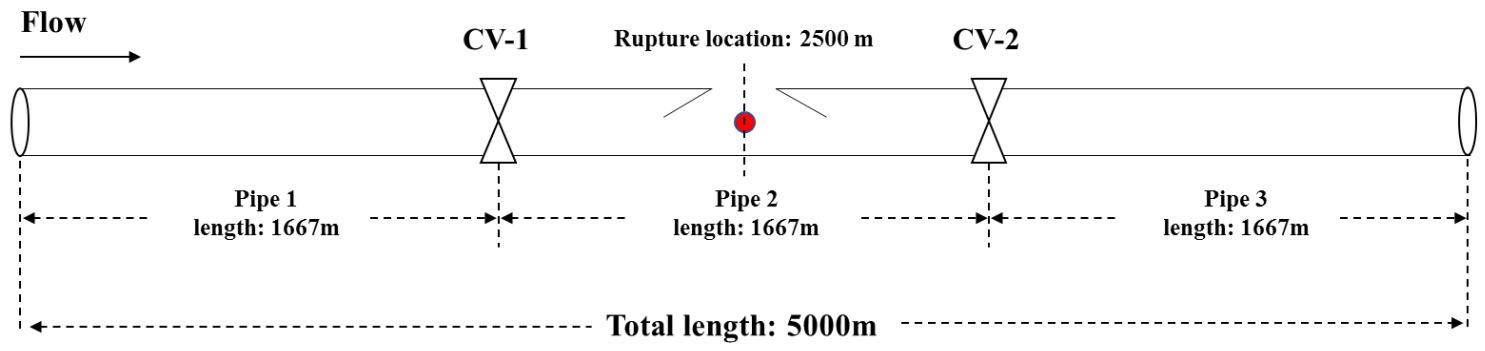

(b)

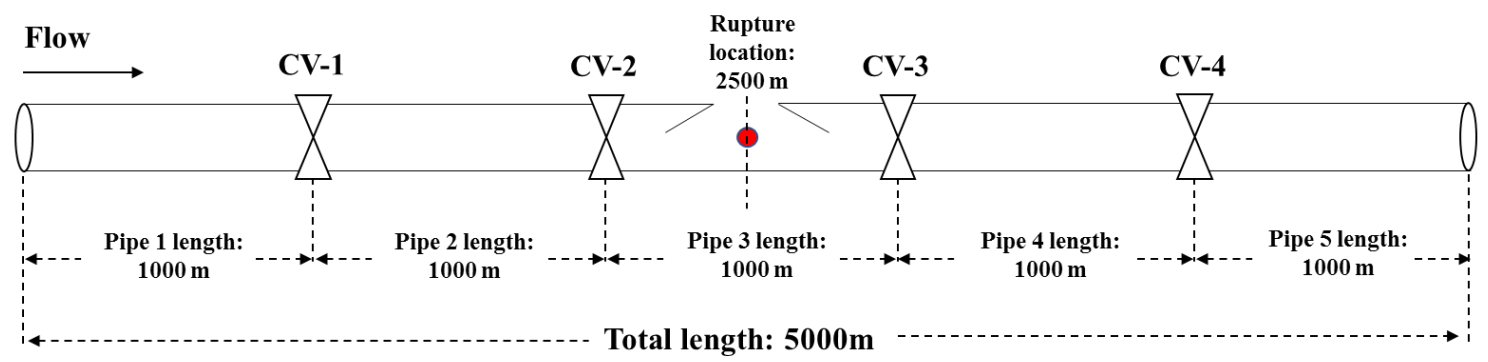

(c)

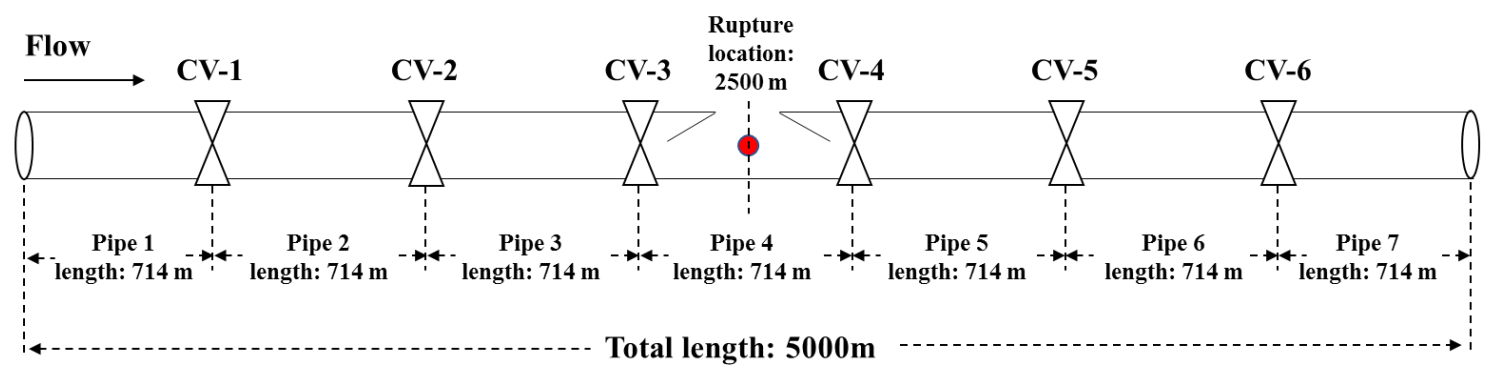

(d)

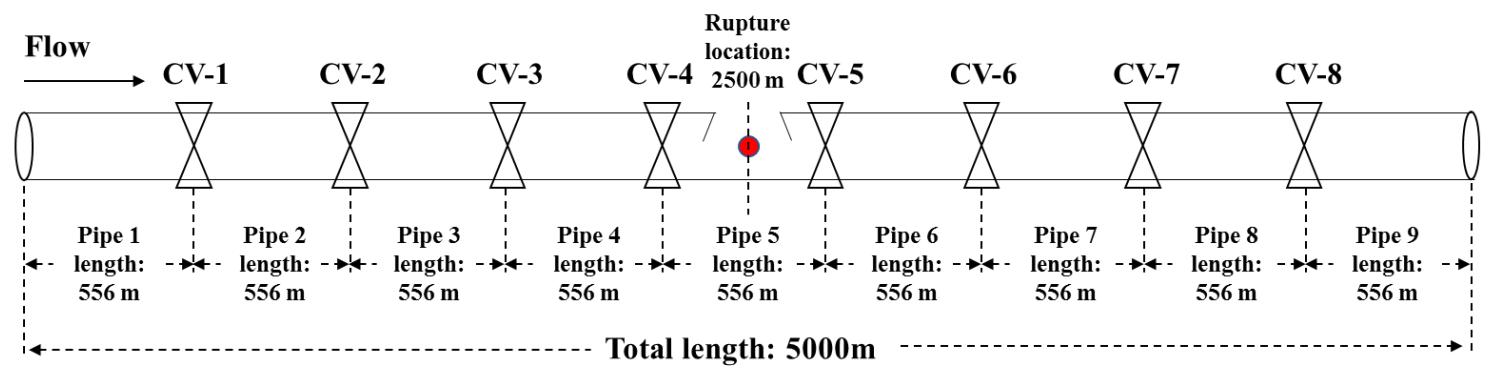

(e)

Figure 5.1: Schematic representation for tests $5.1(a), 5.2(b), 5.3(c), 5.4(d)$ and $5.5(e)$ for scenario 1 (CVs only). 


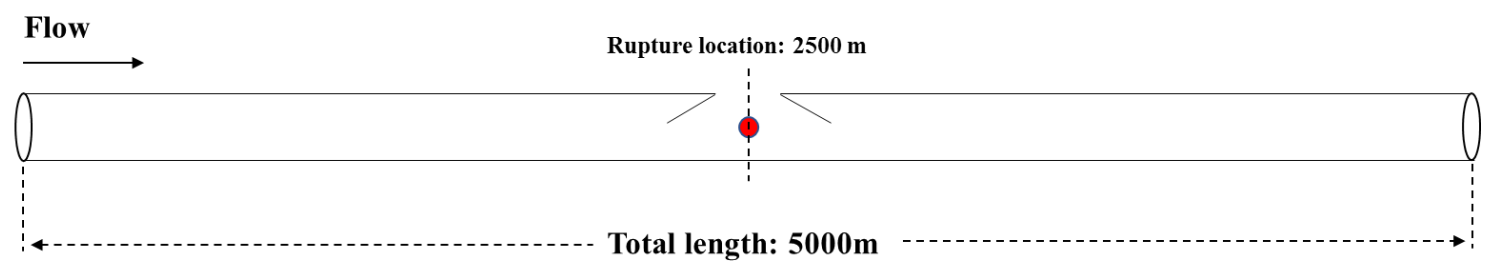

(a)

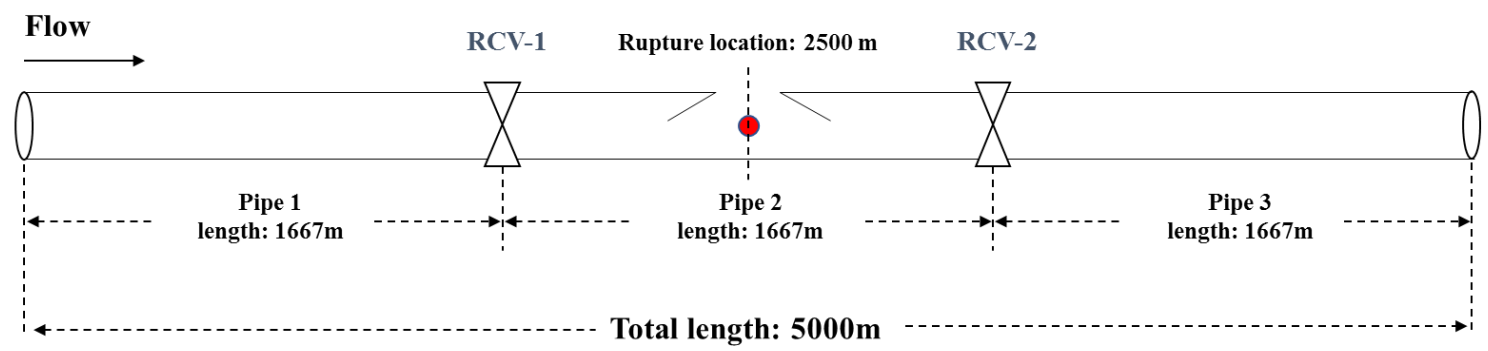

(b)

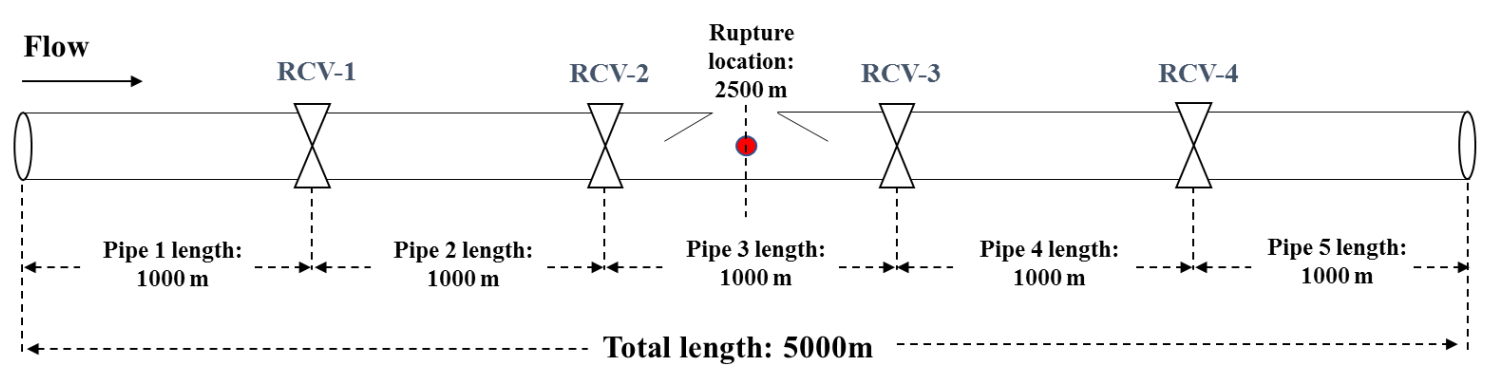

(c)

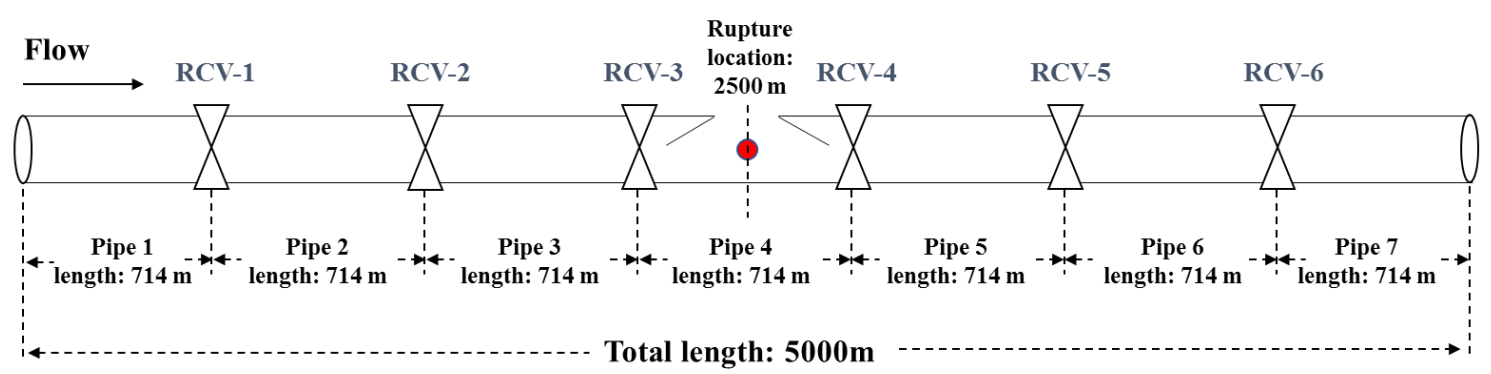

(d)

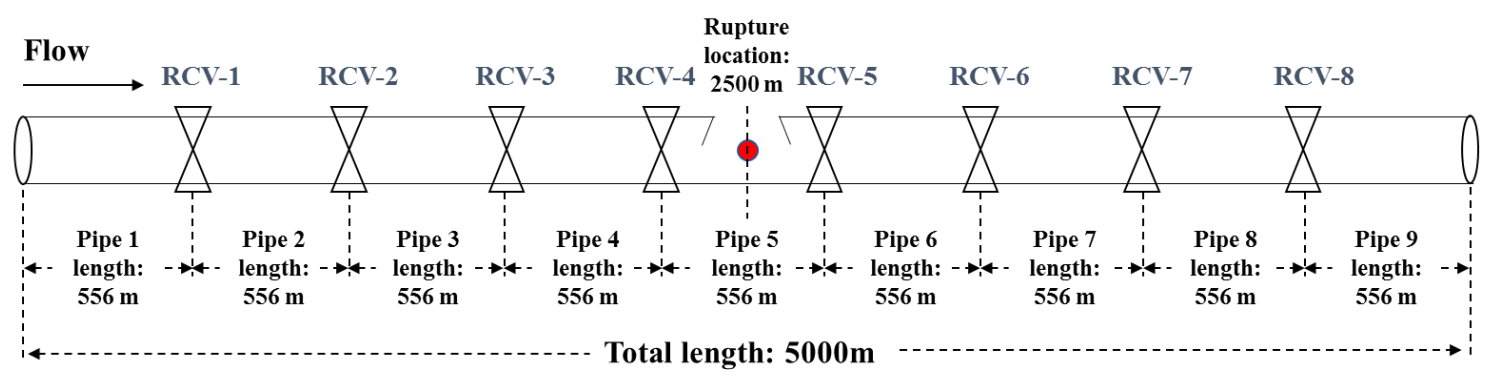

(e)

Figure 5.2: Schematic representation for tests $5.6(a), 5.7(b), 5.8(c), 5.9(d)$ and $5.10(e)$ for scenario 2 (RCVs only). 


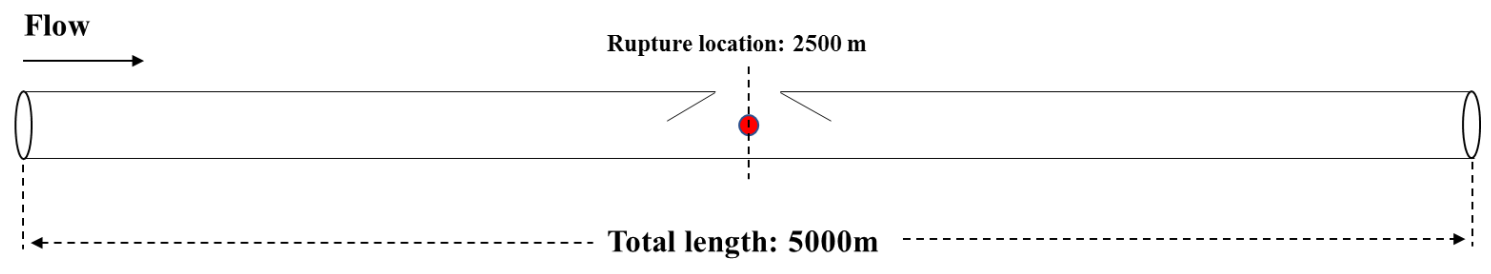

(a)

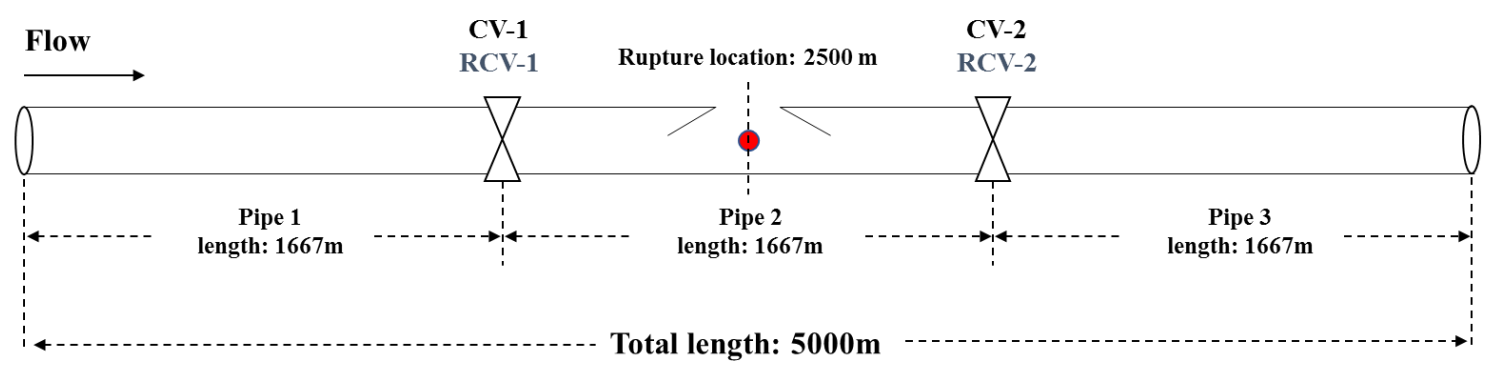

(b)

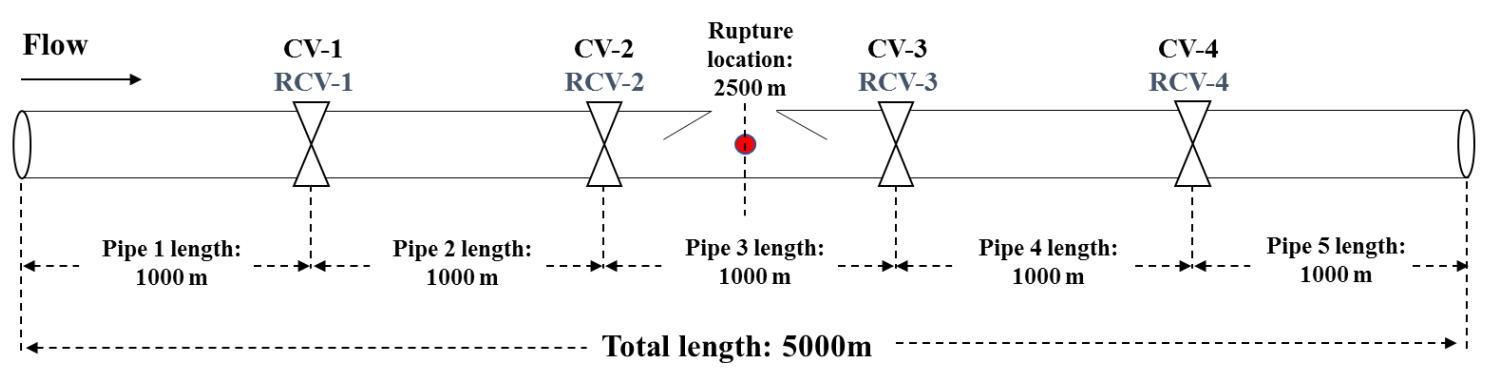

(c)

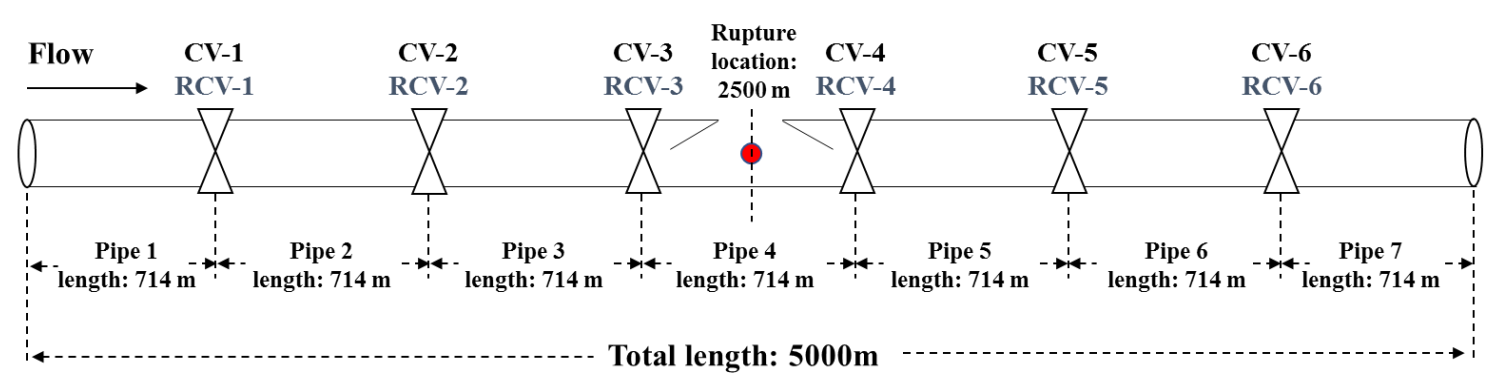

(d)

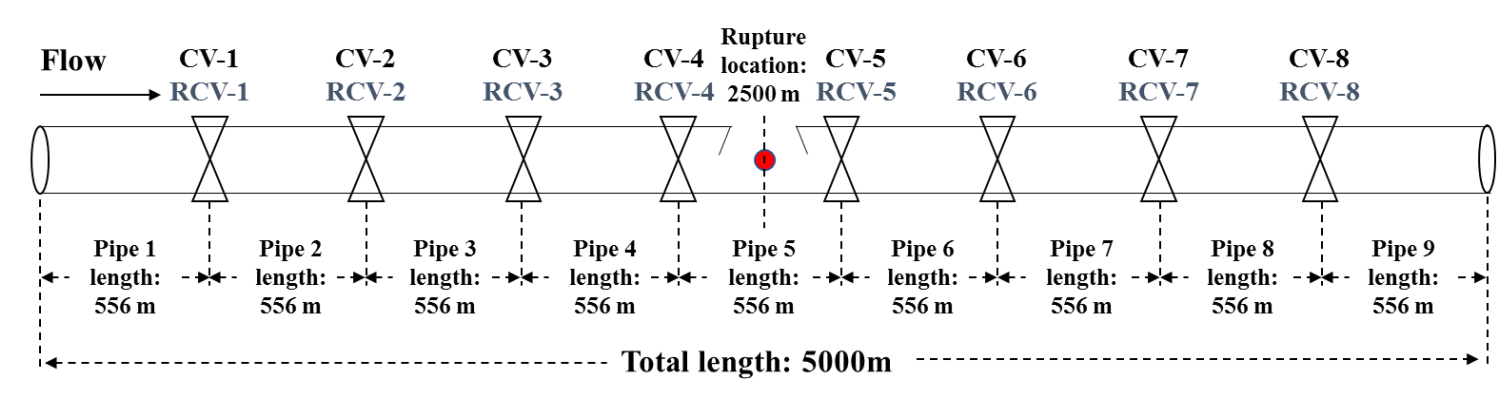

(e)

Figure 5.3: Schematic representation for tests $5.11(a), 5.12(b), 5.13(c), 5.14(d)$ and 5.15 (e) for scenario 3 (CVs and RCVs at same locations; i.e. in tandem). 


\subsubsection{Results and Discussion}

Figures 5.4 to 5.7 respectively show the variations of release pressure, temperature, discharge flowrate and cumulative mass discharged as a function of time for the first $600 \mathrm{~s}$ following the release of $\mathrm{CO}_{2}$ for scenario 1 (tests 5.1 to $5.5, \mathrm{CVs}$ only). In all cases, the time lapsed after complete valve closure following pipeline failure is represented by the vertical dotted line.

Referring to figure 5.4, at the onset of the release, choked flow occurs at the release point, marked by an initial rapid drop in the release pressure (for all 5 tests the release pressure drops from ca. 150 bar to 43 bar). During the decompression, the release pressure remains almost constant (ca. 43 bar) in test 5.1. This can be explained by the infinite reservoir upstream boundary assumption.

For tests 5.2 to 5.5 with $\mathrm{CV}$ installed, a pressure drop can be observed after a short delay following the failure (ca. 43 bar to 30 bar). This is attributed to the closure of the $\mathrm{CV}$ downstream of the puncture at the moment of the flow reversal occurs at the valve location. It should be noted that the CVs upstream of the puncture are not activated. As a result, for tests 5.2 to 5.5, the upstream conditions remain constant and, consequently, the release pressure remains at ca. 30 bar afterwards.

Similar trends can be observed in the release temperature and the discharge flowrate variations with time (figures 5.5 and 5.6 respectively). At the onset of the release, liquid flashingevaporation occurs, leading to a drop in the sonic velocity; this is confirmed by referring to the release flowrate (figure 5.6, the release flowrate drops from ca. $800 \mathrm{~kg} / \mathrm{s}$ to ca. $290 \mathrm{~kg} / \mathrm{s}$ ). The corresponding release temperature (figure 5.5) drops to the saturated value of ca. $280 \mathrm{~K}$.

Referring to figure 5.7, in all cases, the cumulative mass discharged increases linearly with time. Comparison of all tests demonstrates that the amount of cumulative mass discharged is significantly reduced with the installation of CVs (cf. test 5.1 and tests 5.2 to 5.5). However, as can be clearly seen in figure 5.7, the introduction of any additional CVs has minimal impact on the cumulative mass discharged. This is due to the fact that as valve spacing decreases, the inventory encapsulated between the release point and the most adjacent $\mathrm{CV}$ downstream $(\mathrm{CV}$ $2,3,4$ or 5) is reduced. However, due to the infinite reservoir conditions upstream of the release point, such a reduction is insignificant as compared to the cumulative mass discharged. 


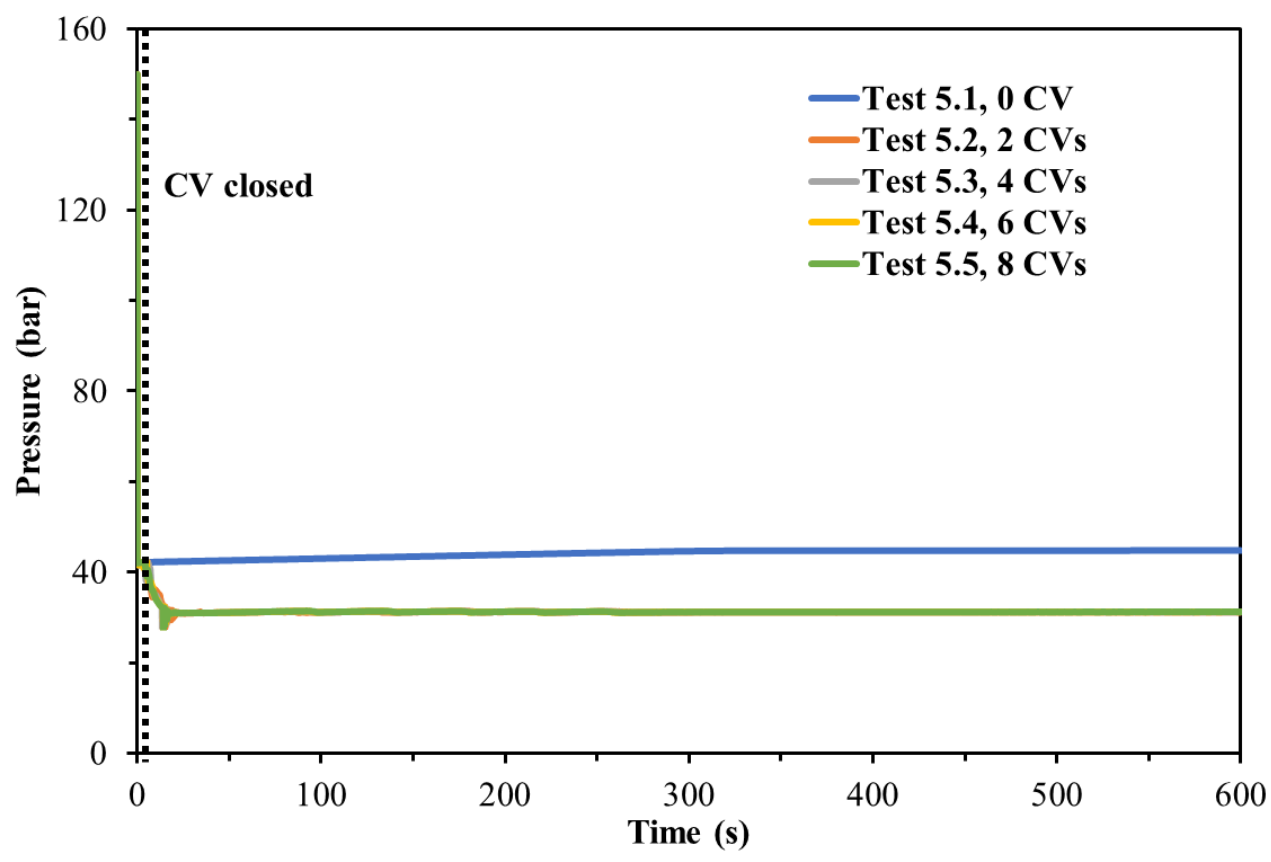

Figure 5.4: Tests 5.1 to 5.5 variation of release pressure with time.

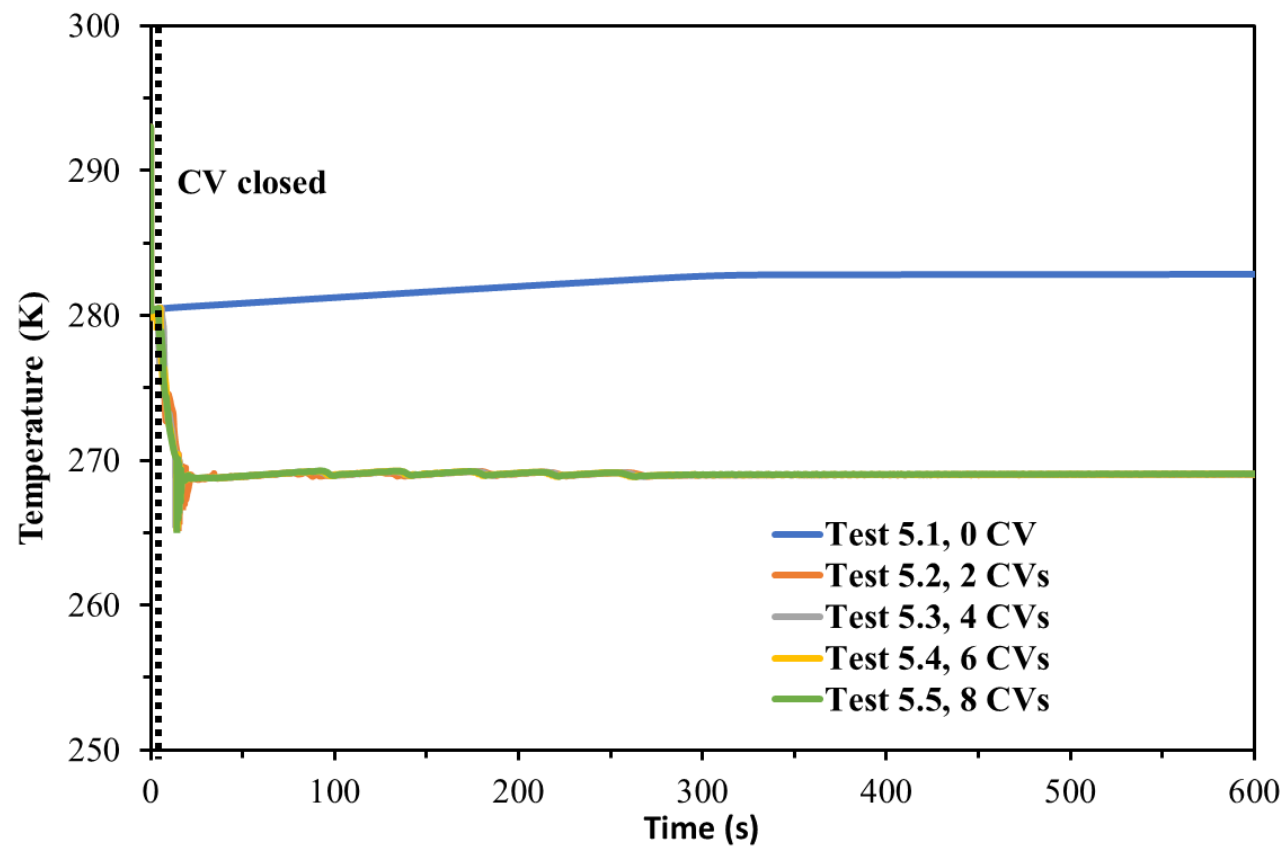

Figure 5.5: Tests 5.1 to 5.5 variation of release temperature with time. 


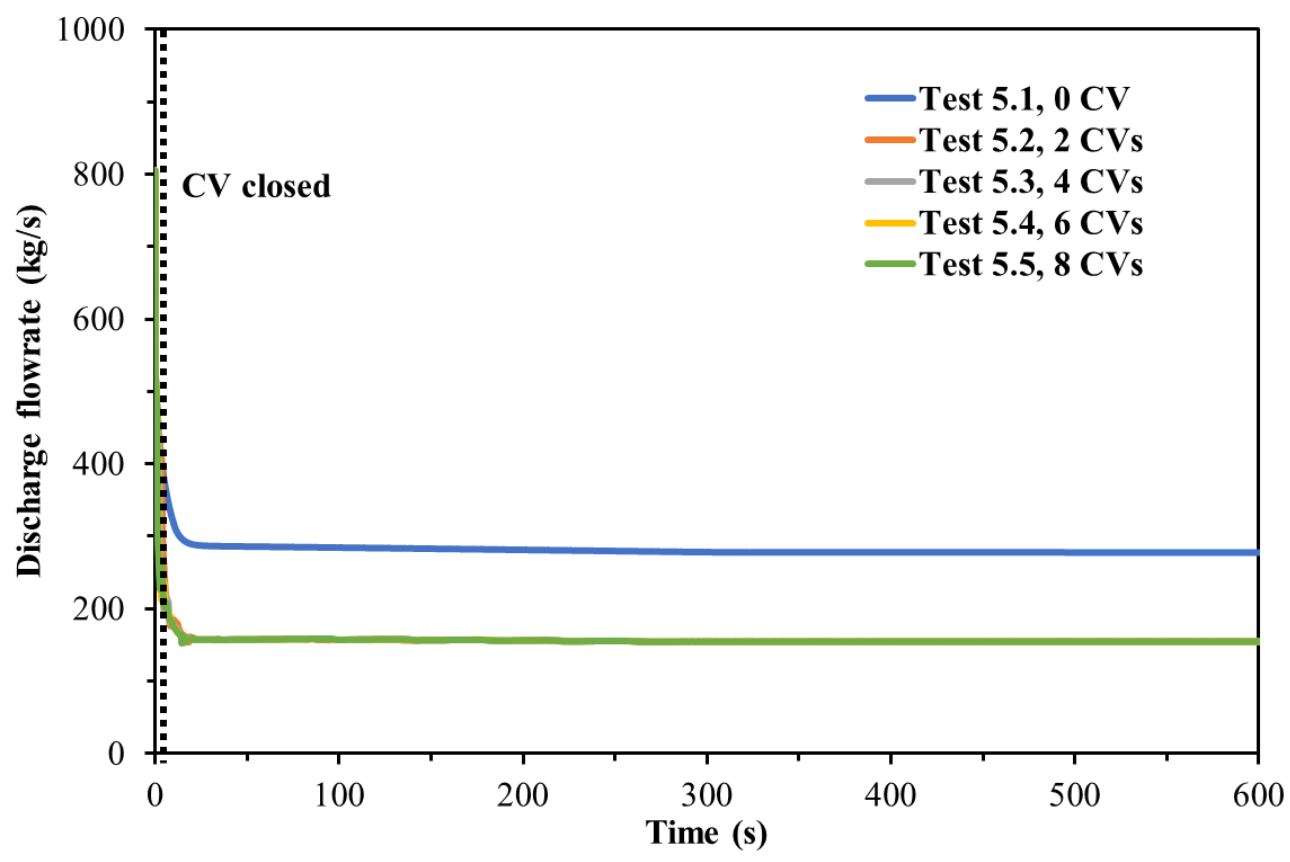

Figure 5.6: Tests 5.1 to 5.5 variation of discharge flowrate with time.

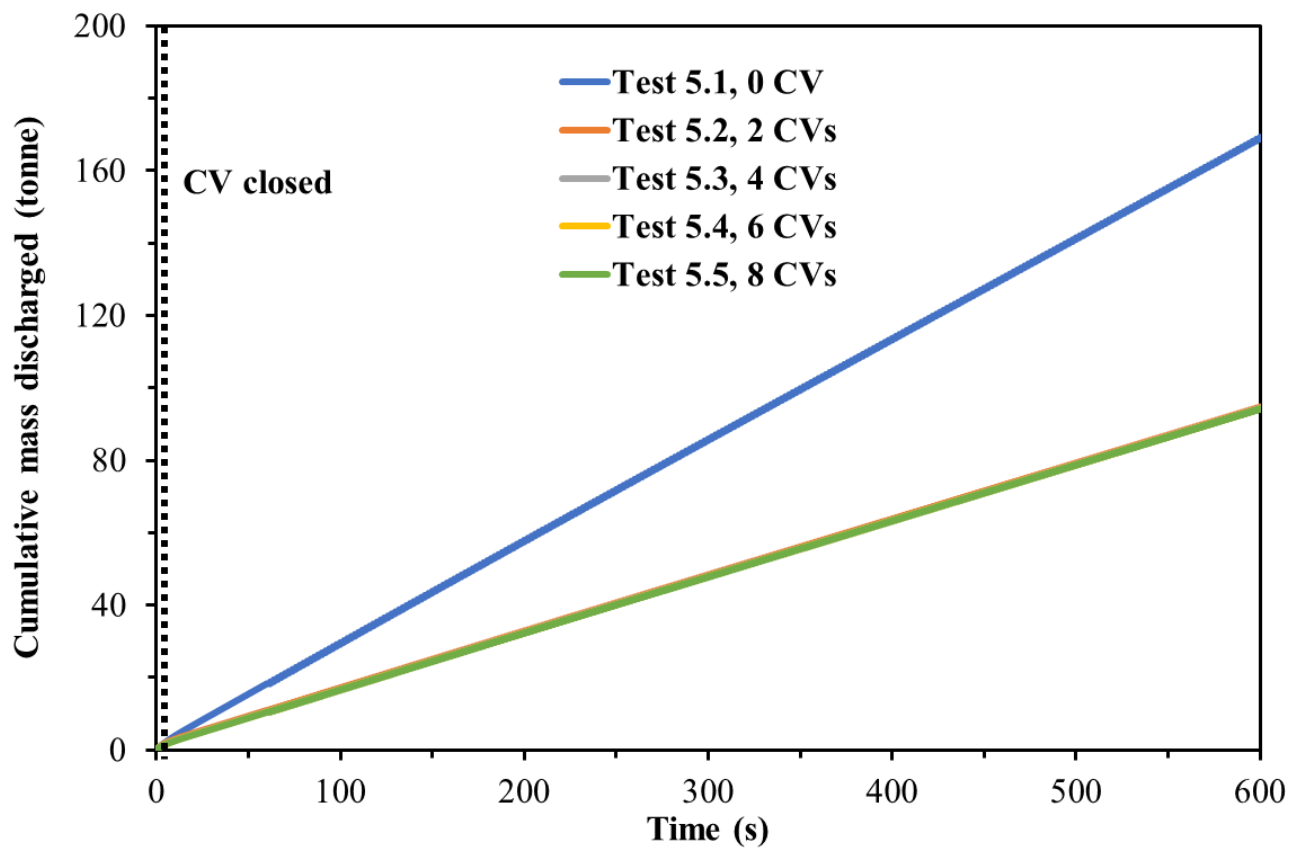

Figure 5.7: Tests 5.1 to 5.5 variation of cumulative mass discharged with time. 
Figures 5.8 to 5.11 respectively show the variations of the release pressure, temperature, flowrate, and the cumulative mass discharged as a function of time for the first $600 \mathrm{~s}$ following the release for scenario 2 (tests 5.6 to 5.10).

As it may be observed, in all cases, prior to valve closure (specified by the valve activation time of $120 \mathrm{~s}$ ), the same trends as predicted for scenario 1 (tests 5.1 to 5.5) are observed. However, upon RCV activation, the observed variations of the relevant flow parameters such as pressure, temperature and discharge flow rate with time change drastically. This observation can be explained by the fact that at the time of valve activation (120 s following the decompression), both the upstream and downstream of the release point are isolated by the corresponding RCVs (see figures 5.2b to 5.2e). As a result, there is no longer a feed flow from the upstream infinite reservoir. Whence, the release pressure continuously drops during release and reaches the ambient pressure (1.01 bar) as shown in figure 5.8.

Turning to the temperature predictions (figure 5.9), a similar trend in the data as compared to the pressure/time behaviour can be observed prior to complete depressurisation. Afterwards, the remaining inventory is in vapour phase, manifested in the observed temperature recovery (at ca. 340, 380 and 456 s respectively for tests 5.7, 5.8 and 5.9) due to heat transfer from the surrounding ambient to the fluid through the pipe wall.

With respect to the discharge flowrate (figure 5.10), as expected, same trend in the data as compared to the release pressure (figure 5.8) is seen throughout the decompression.

Turning to the variation of the cumulative mass discharged throughout the entire simulated decompression process (figure 5.11), the cumulative mass discharged increases linearly for test 5.6 where no valve is installed. For tests 5.7 to 5.10 with different numbers of RCVs installed along the pipeline, a reduction in the amount of the cumulative mass discharged can be seen after valve closure. Following this, no further loss of the inventory is observed after the complete decompression of the isolated section in the test pipe. It is noteworthy that unlike the case with CVs, an increase in the number of the RCVs installed along the pipe (hence the reduced valve spacing) presents an effective reduction of the cumulative mass discharged. However, such reduction obtained by increasing the number of valves becomes marginal above a certain range (e.g. the difference in the cumulative mass discharged between tests 5.8 (4 RCVs) and 5.10 (8 RCVs) is less than $3 \%$ ). This is due to the relatively long valve activation 
time (120 s in this case), which results in significant amount of inventory loss before RCVs starting to close.

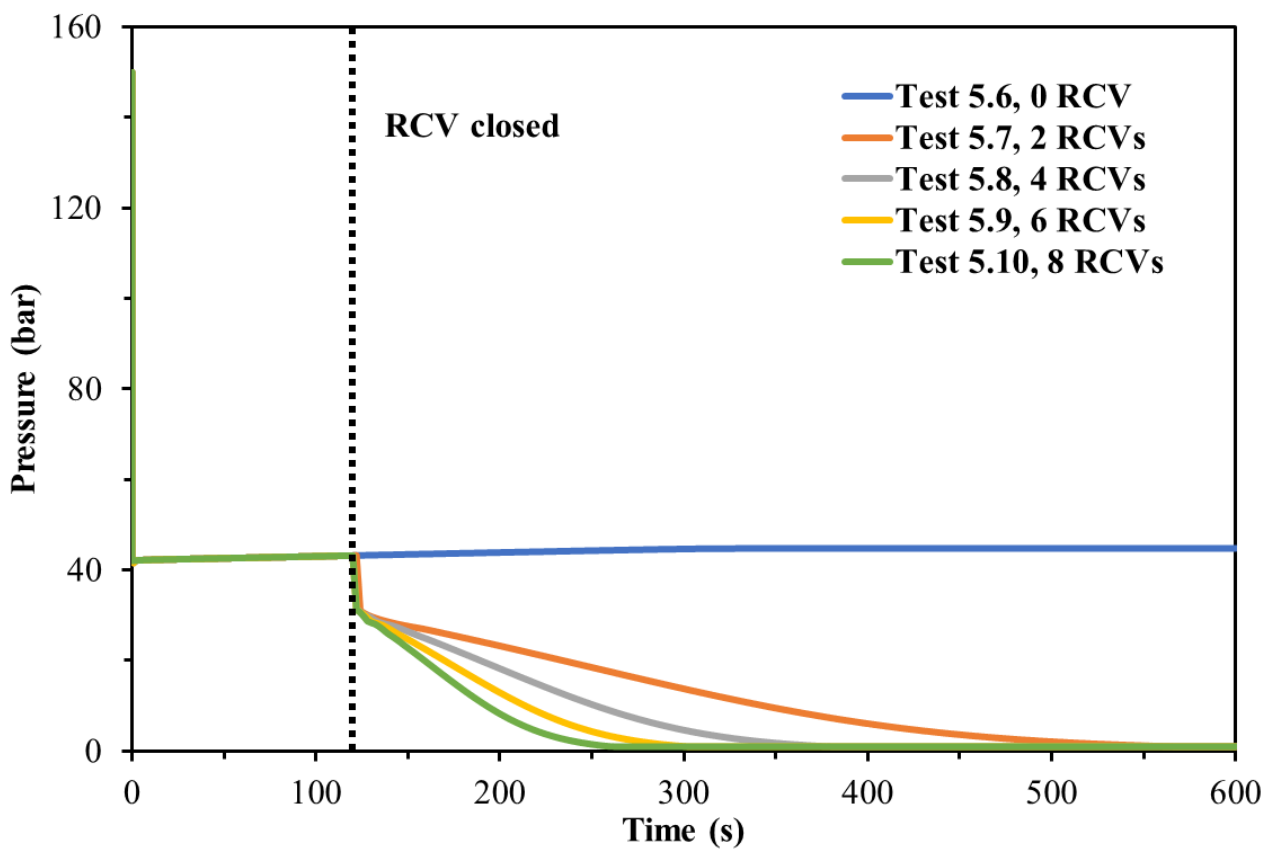

Figure 5.8: Tests 5.6 to 5.10 variation of release pressure with time.

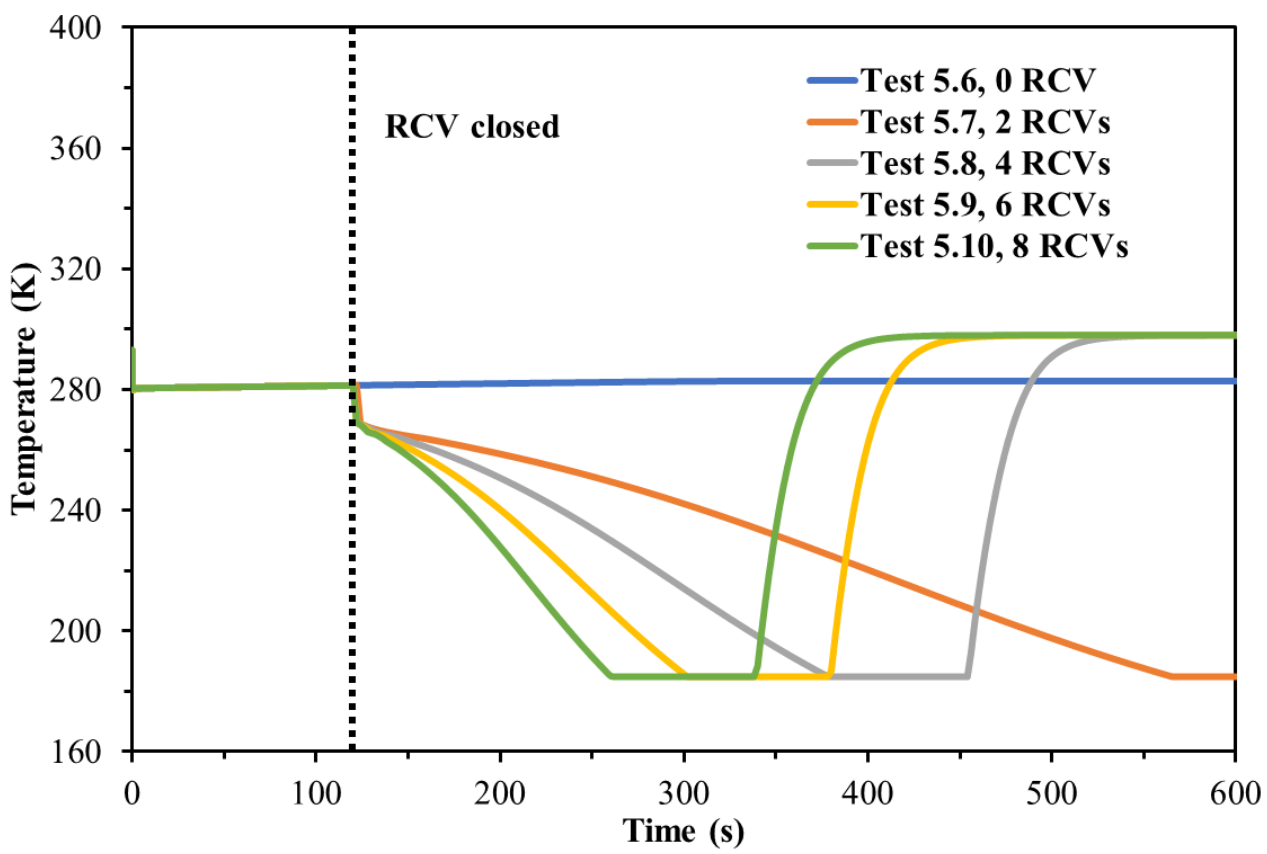

Figure 5.9: Tests 5.6 to 5.10 variation of release temperature with time. 


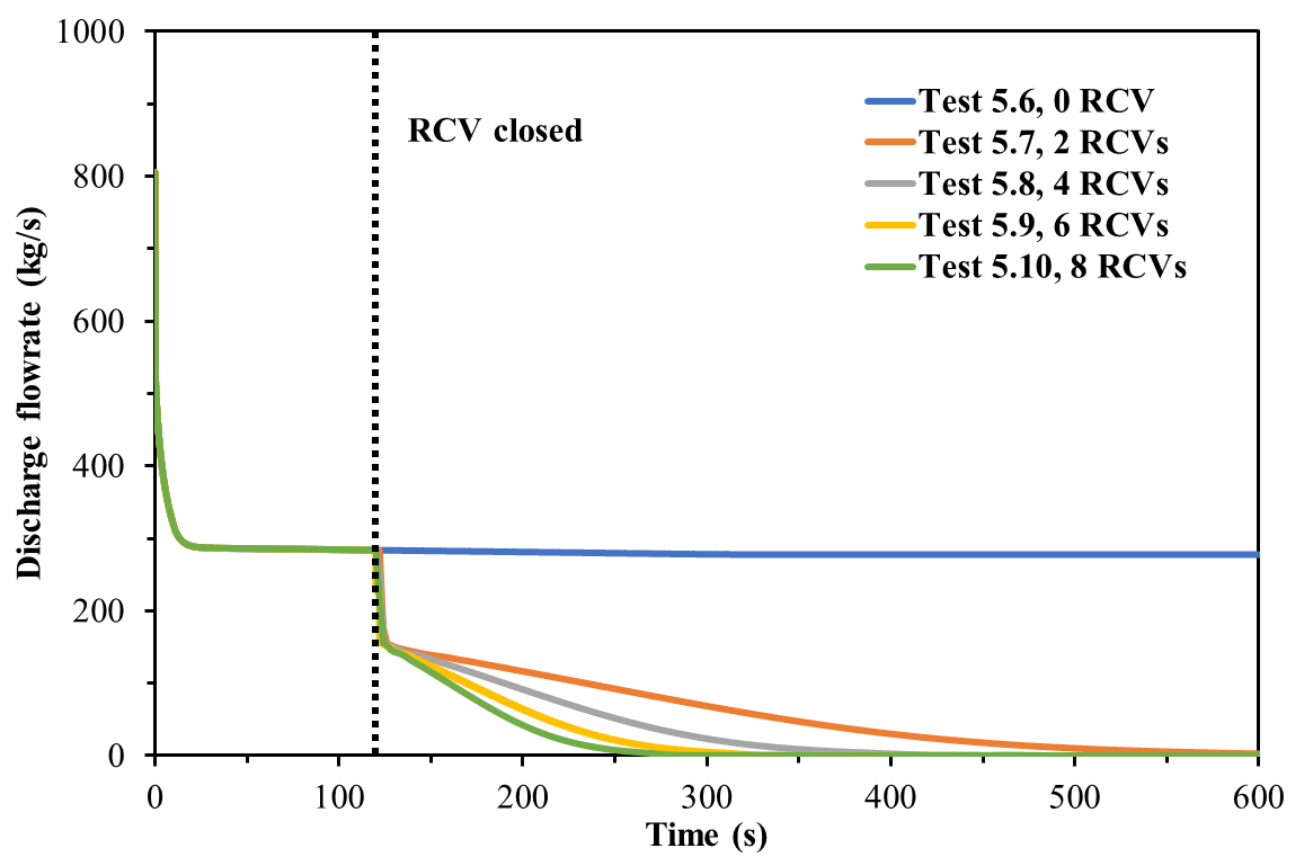

Figure 5.10: Tests 5.6 to 5.10 variation of discharge flowrate with time.

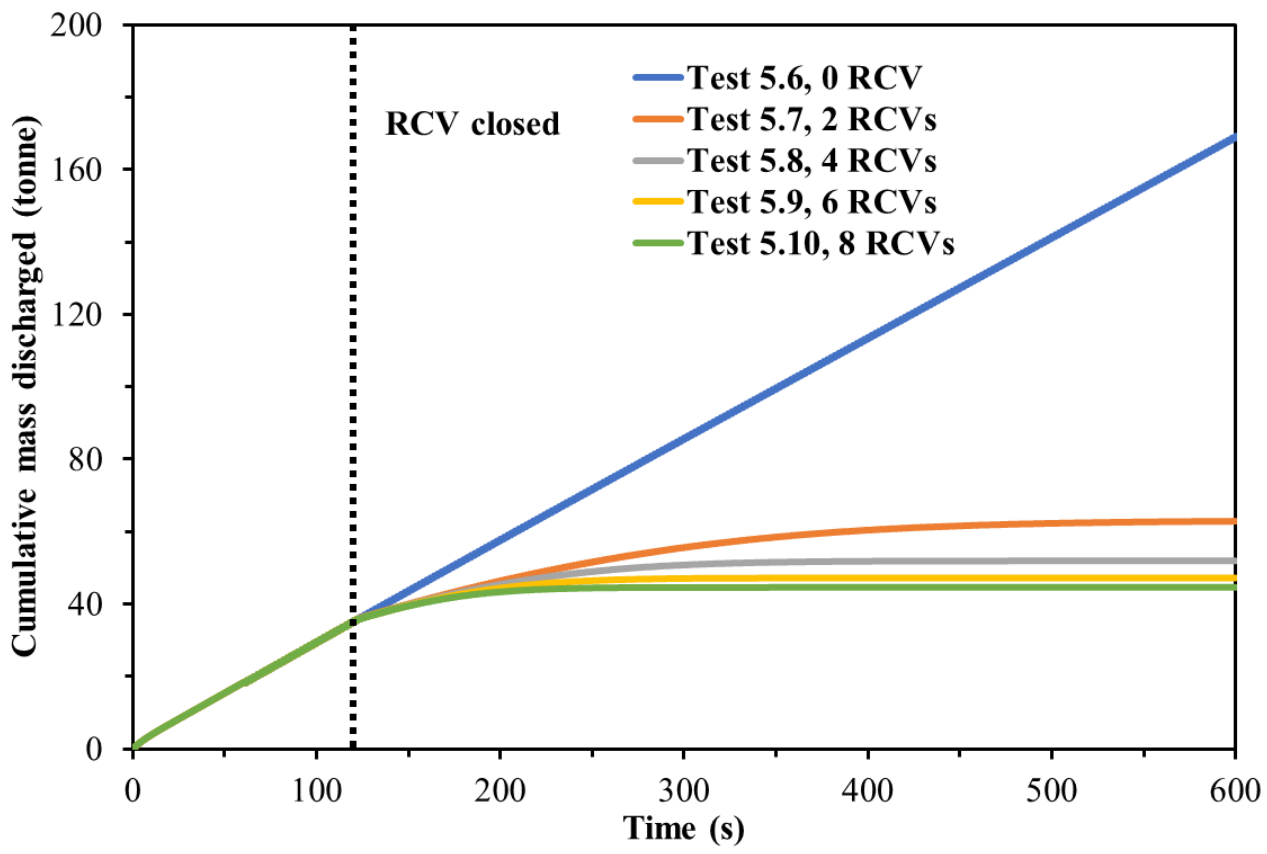

Figure 5.11: Tests 5.6 to 5.10 variation of cumulative mass discharged with time.

Figures 5.12 to 5.15 respectively show the variations of the release pressure, temperature, flowrate, and the cumulative mass discharged as a function of time during decompression for test scenario 3 (tests 5.11 to 5.15), where both CVs and RCVs are installed at the same locations 
(i.e. in tandem). In figures 5.12 to 5.15, the trends in the release pressure, temperature, flowrate and the cumulative mass discharged as a function of time are the same as those for scenarios 1 and 2, thus no further discussion is needed.

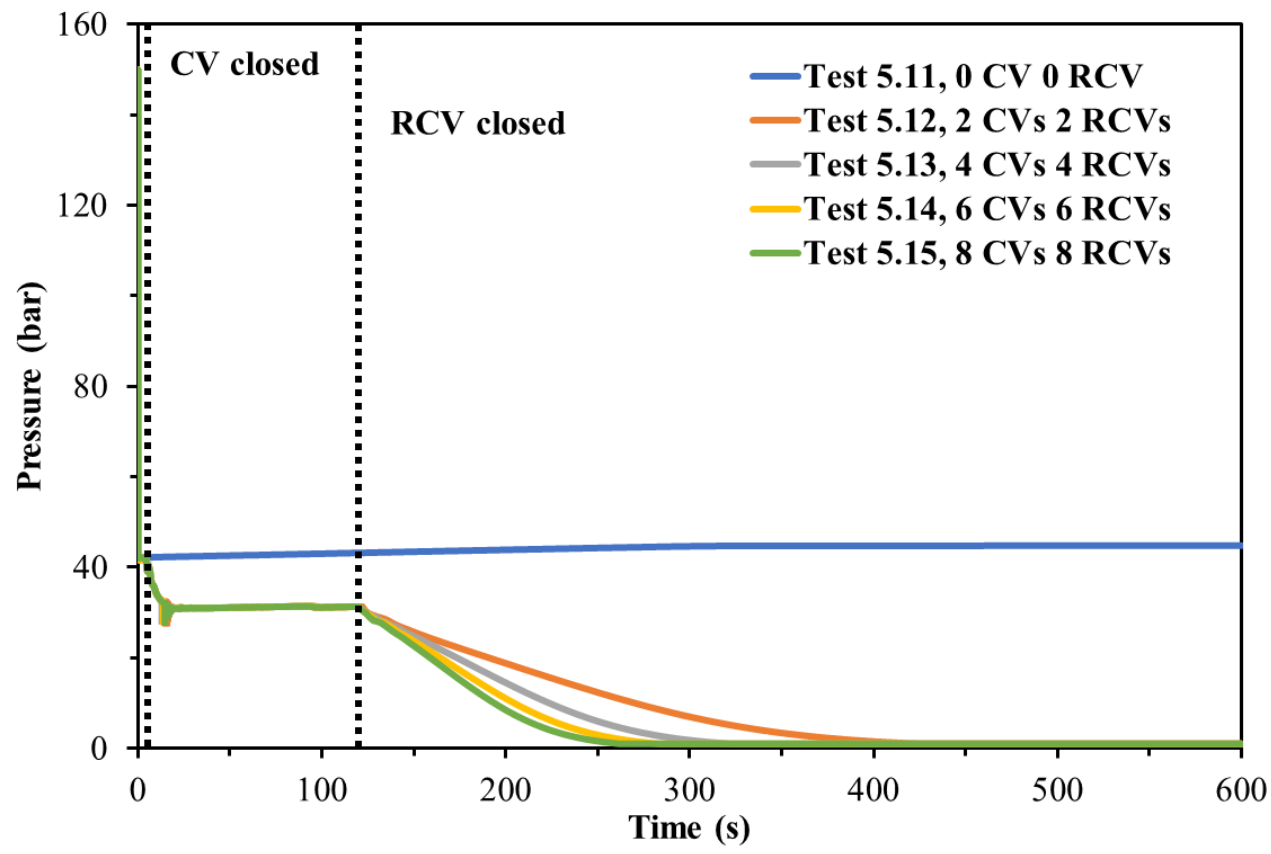

Figure 5.12: Tests 5.11 to 5.15 variation of release pressure with time.

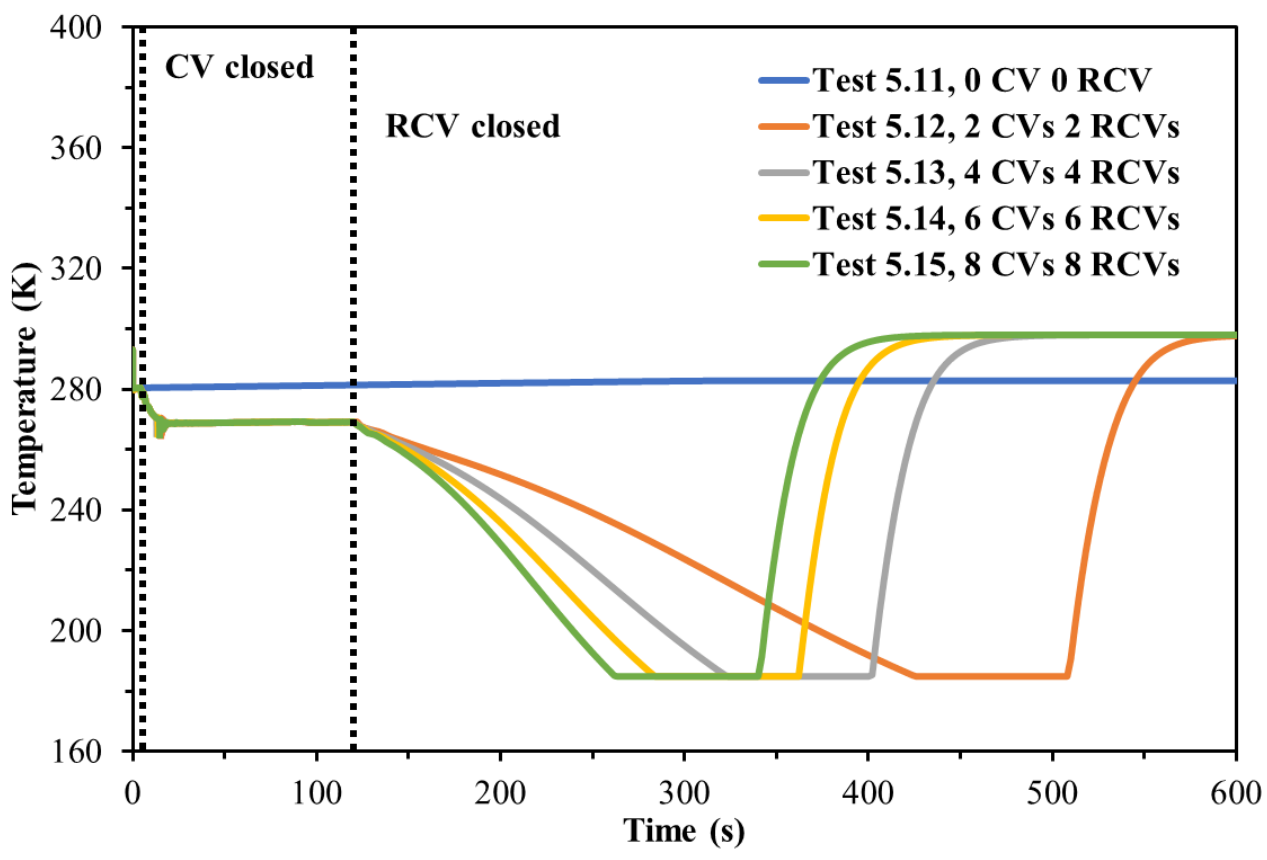

Figure 5.13: Tests 5.11 to 5.15 variation of release temperature with time. 


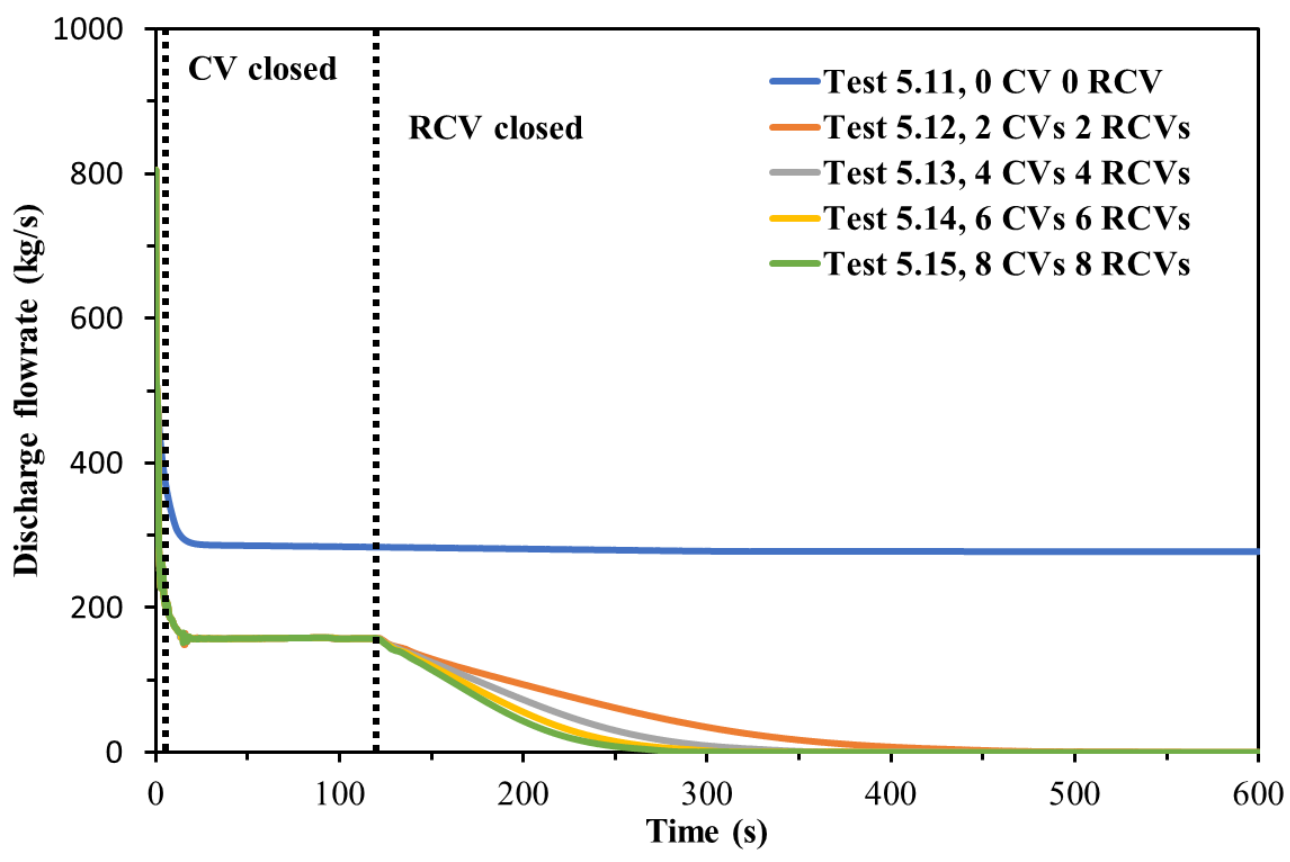

Figure 5.14: Tests 5.11 to 5.15 variation of discharge flowrate with time.

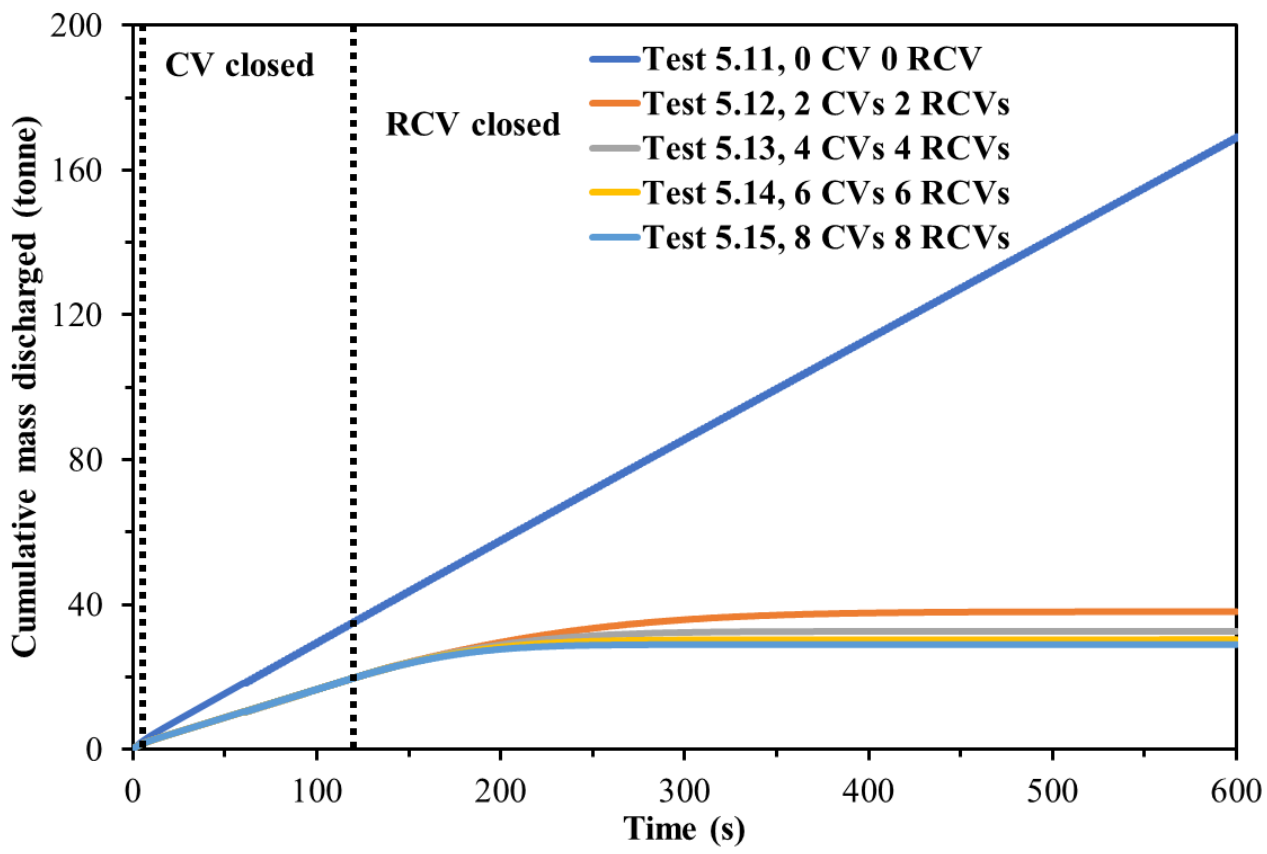

Figure 5.15: Tests 5.11 to 5.15 variation of cumulative mass discharged with time.

To further demonstrate the impact of the installation of both CVs and RCVs at the same locations (i.e. in tandem), the predictions of the cumulative mass discharged predictions from 
test 5.1 (no valves installed; see figure 5.1), test 5.5 (8 equally-spaced CVs; see figure 5.1), test 5.10 (8 equally-spaced RCVs; see figure 5.2) and test 5.12 (2 RCVs and 2 CVs; see figure 5.3) are presented in figure 5.16 .

From figure 5.16, it is clear that test 5.12 (with $2 \mathrm{RCVs}$ and $2 \mathrm{CVs}$ ) shows the best performance in terms of the cumulative mass discharged at $600 \mathrm{~s} \mathrm{(ca.} 38$ tonne) compare to the other valve configurations (ca. 45 tonne for test 5.10; ca. 94 tonne for test 5.5 and ca. 169 tonne for test 5.1) considered. This is due to the instantaneous response of the CV downstream of the release point upon detecting flow reversal, as well as the RCV upstream which stops the feed flow from the infinite reservoir upstream.

As discussed earlier, increasing the number of same type of valves (hence reducing the valve spacing) above a certain range ( $2 \mathrm{CVs}$ or $4 \mathrm{RCVs}$ ) has marginal reduction in the cumulative mass discharged. As such, the above results indicate the potential merits in the choice of using $\mathrm{CV}$ and $\mathrm{RCV}$ at the same locations (i.e. in tandem) for ESDV optimisation, rather than increasing the number of same type of valves above a certain range.

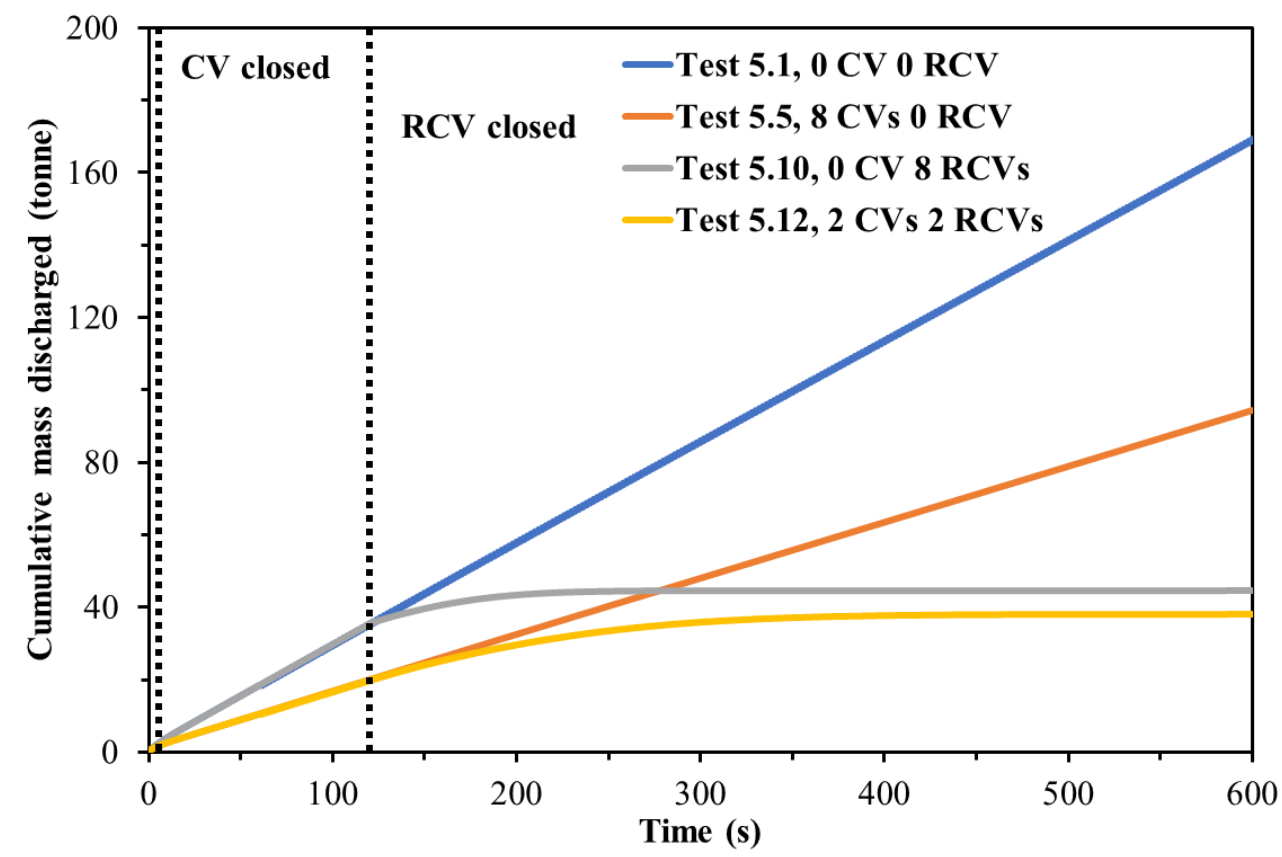

Figure 5.16: Comparison of tests 5.1, 5.5, 5.10 and 5.12 variation of cumulative mass discharged with time. 


\subsection{The Study of the Choice of ESDVs on Outflow following a Pipeline Full Bore Rupture}

\subsubsection{Simulation Test Setup}

In this section, the efficacies of ASVs, RCVs and CVs and their combinations are assessed by determining the amount of total inventory loss prior to complete isolation following ethylene pipeline FBR failure. The simulations are based on a hypothetical $15 \mathrm{~km}$ length, $250 \mathrm{~mm}$ i.d., thermally insulated (heat transfer coefficient $=6 \mathrm{~kW} / \mathrm{m}^{2} \mathrm{~K}$ ) carbon steel pipeline, with wall thickness of $12 \mathrm{~mm}$, transporting supercritical state ethylene initially at 90 bar and $278 \mathrm{~K}$, representative of a typical ethylene onshore pipeline. All relevant details regarding the pipeline setup are summarised in table 5.2.

Table 5.2: Initial conditions for the ethylene pipeline tests.

\begin{tabular}{|l|l|l|}
\hline \multicolumn{2}{|l|}{ Input } & Value \\
\hline \multirow{5}{*}{ Pipeline } & Pipeline outer diameter (mm) & 274 \\
\cline { 2 - 3 } & Pipeline wall thickness (mm) & 12 \\
\cline { 2 - 3 } & Pipeline roughness height (mm) & 0.005 \\
\cline { 2 - 3 } Type of failure & Pipeline length (km) & 15 \\
\cline { 2 - 3 } Boundary conditions & Pipeline inclination ( $\left.{ }^{\circ}\right)$ & 0 \\
\hline \multirow{5}{*}{ Initial conditions } & Type of failure & FBR in the middle \\
\hline & Upstream boundary condition & Constant \\
(Reservoir)
\end{tabular}


Figure 5.17 schematically shows the ethylene pipeline section with two ESDVs installed along the pipeline. The valve spacing, $D$ is assumed to be 1 or $5 \mathrm{~km}$. FBR is assumed to occur at a distance, $x$ downstream of the first valve (ESDV-1).

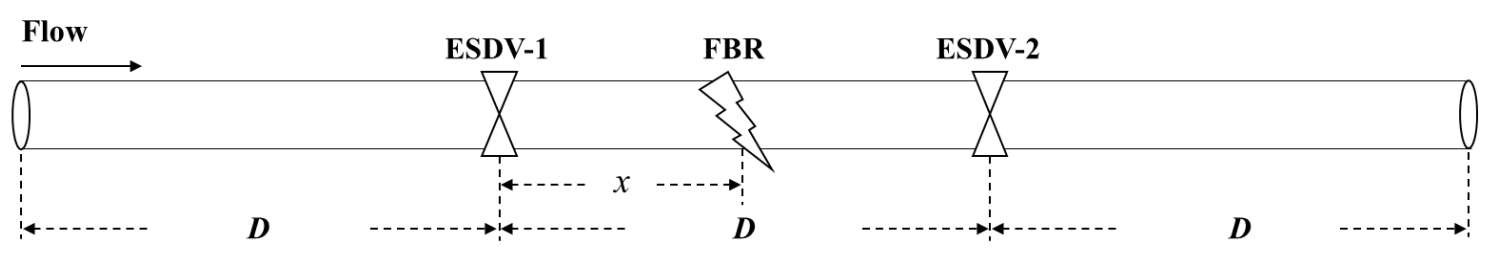

Figure 5.17: Schematic representation of the ethylene pipeline section indicating the location of two ESDVs relative to failure point.

Four simulation tests are carried out, as listed in table 5.3. In tests 5.16 and 5.18, ESDV-1 and ESDV-2 are of the same type, while in tests 5.17 and 5.19, CV is used as the downstream valve (ESDV-2). The assumed FBR location $x / D$ and valve characteristics, including the linear closure rate, ASV activation pressure (i.e. the pressure drop from line pressure), $\Delta p_{a}$ and RCV activation time, $t_{d}$ are also presented in table 5.3.

Table 5.3: FBR Location, types and characteristics of ESDV setup in the pipeline section (figure 5.17) for tests 5.16 to 5.19 .

\begin{tabular}{|c|c|c|c|c|c|}
\hline \multirow{2}{*}{$\begin{array}{l}\text { Test } \\
\text { No. }\end{array}$} & \multirow{2}{*}{$\begin{array}{l}\text { FBR Location } \\
\qquad(x / D)\end{array}$} & \multicolumn{2}{|c|}{ ESDV-1 } & \multicolumn{2}{|c|}{ ESDV-2 } \\
\hline & & Valve type & $\begin{array}{c}\text { Valve } \\
\text { characteristics }\end{array}$ & Valve type & $\begin{array}{c}\text { Valve } \\
\text { characteristics }\end{array}$ \\
\hline 5.16 & $0.1,0.5,0.9$ & ASV & $\begin{array}{l}\text { valve linear closure } \\
\text { rate }=2.54 \mathrm{~cm} / \mathrm{s} \\
\qquad \Delta p_{a}=10 \mathrm{bar}\end{array}$ & ASV & $\begin{array}{c}\text { valve linear } \\
\text { closure rate }=2.54 \\
\mathrm{~cm} / \mathrm{s}, \Delta p_{a}=10 \mathrm{bar}\end{array}$ \\
\hline 5.17 & $0.1,0.5,0.9$ & ASV & $\begin{array}{l}\text { valve linear closure } \\
\text { rate }=2.54 \mathrm{~cm} / \mathrm{s} \\
\qquad \Delta p_{a}=10 \mathrm{bar}\end{array}$ & $\mathrm{CV}$ & $\begin{array}{l}\text { Instantaneous } \\
\text { closure }\end{array}$ \\
\hline 5.18 & $0.1,0.5,0.9$ & $\mathrm{RCV}$ & $\begin{array}{c}\text { valve linear closure } \\
\text { rate }=2.54 \mathrm{~cm} / \mathrm{s} \\
t_{a}=240 \mathrm{~s}\end{array}$ & $\mathrm{RCV}$ & $\begin{array}{c}\text { valve linear } \\
\text { closure rate }=2.54 \\
\mathrm{~cm} / \mathrm{s}, t_{a}=240 \mathrm{~s}\end{array}$ \\
\hline 5.19 & $0.1,0.5,0.9$ & $\mathrm{RCV}$ & $\begin{array}{c}\text { valve linear closure } \\
\text { rate }=2.54 \mathrm{~cm} / \mathrm{s} \\
t_{a}=240 \mathrm{~s}\end{array}$ & $\mathrm{CV}$ & $\begin{array}{l}\text { Instantaneous } \\
\text { closure }\end{array}$ \\
\hline
\end{tabular}




\subsubsection{Results and Discussion}

Figure 5.18 shows the total inventory loss (i.e. the sum of the mass released from the pipeline prior to complete valve closure and the amount of inventory remaining in the isolated section of the pipe at the moment of complete valve closure, calculated by equation 4.3), $M_{\text {total }}$ versus FBR location, $x / D$ for tests 5.16 and 5.17 (see table 5.3) for the valve spacing distances, $D$ of 1 (figure 5.18 a) and $5 \mathrm{~km}$ (figure 5.18b). As it may be observed, for both valve spacings, the total inventory losses for test 5.16 (ASVs only) are nearly the same as for FBR located close to either of the valves (i.e. $x / D=0.1$ or 0.9 ) and attain minima at $x / D=0.5$. This can be explained by the fact that initial velocity of the flow in the pipe $(1 \mathrm{~m} / \mathrm{s})$ is relatively small compared with the speed of the expansion wave propagation along the pipe. As such, valve activation time and the corresponding inventory losses are mainly affected by the distance between the valve and the rupture plane, but not the initial feed flow prior to failure.

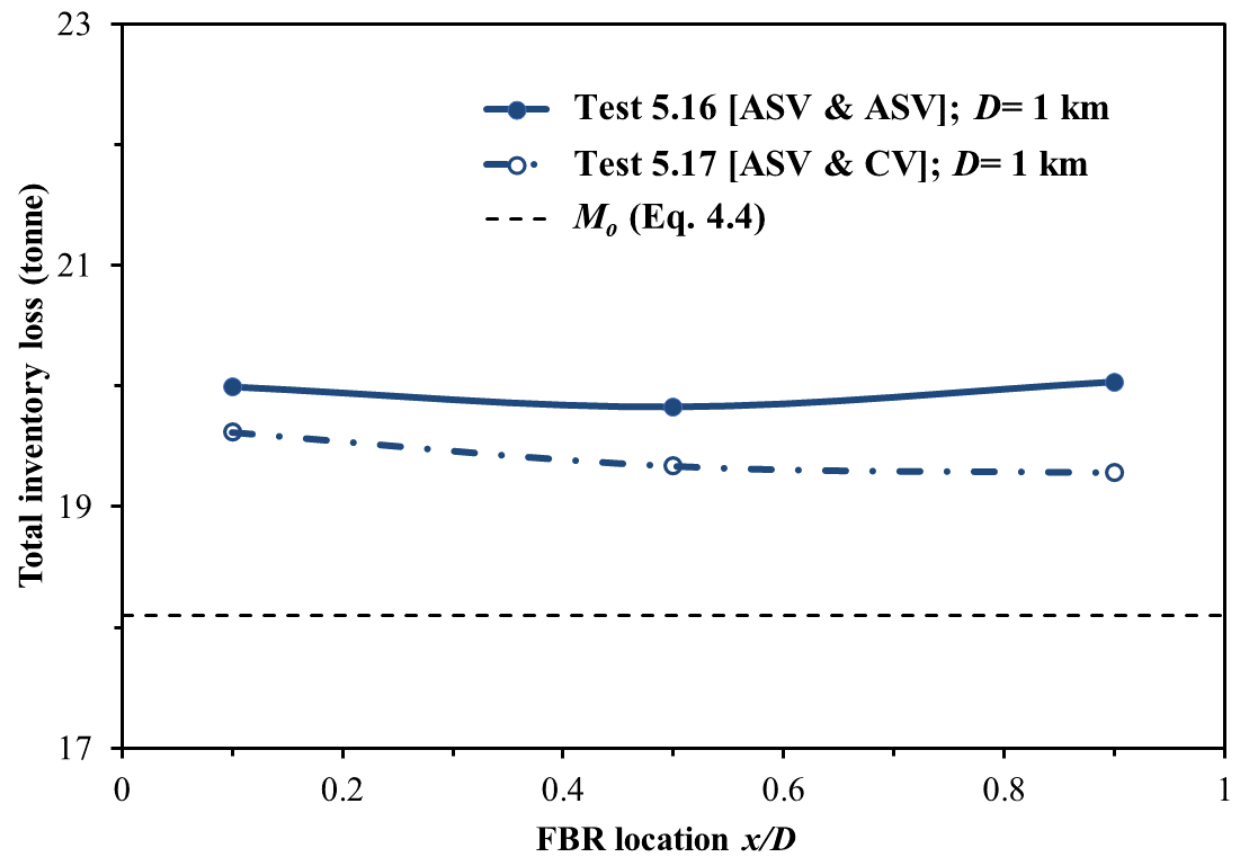

(a) 


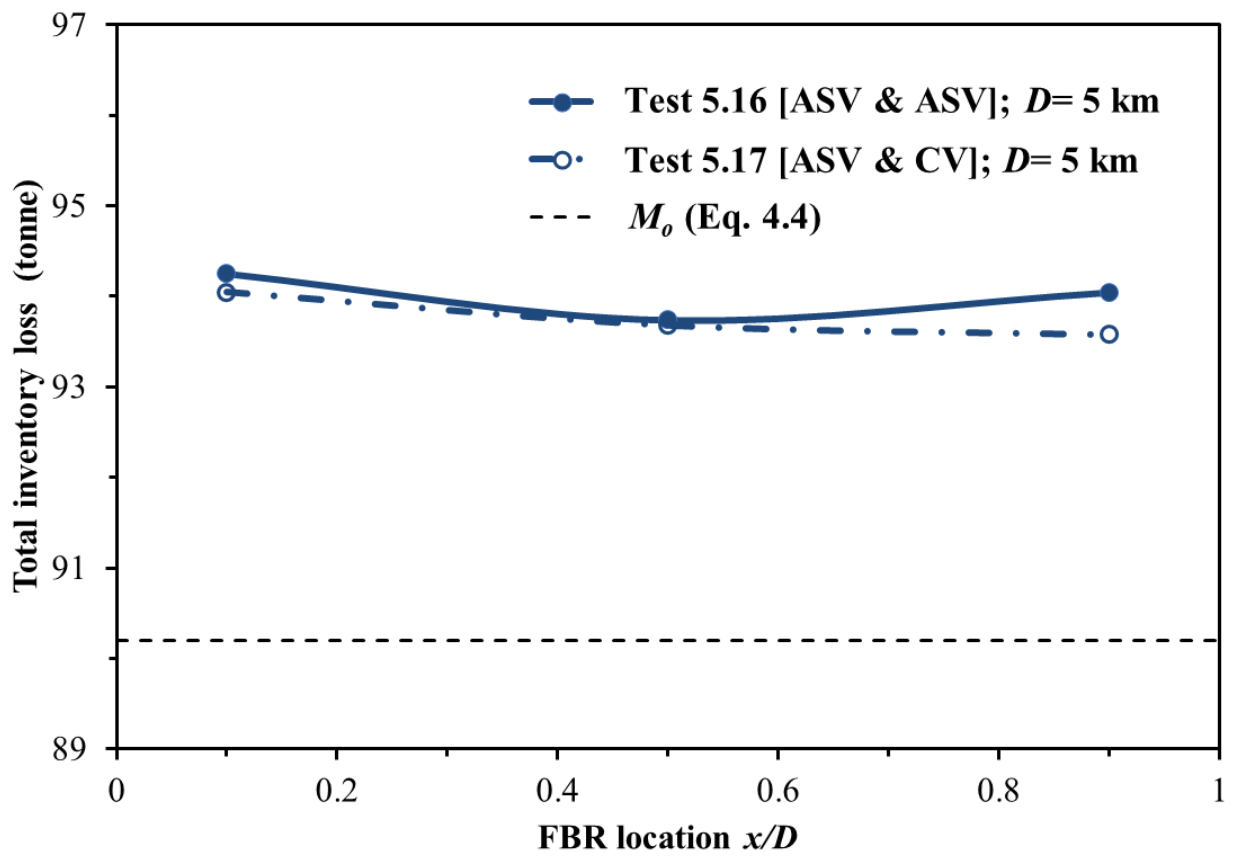

(b)

Figure 5.18: Variation of the total inventory loss as a function of FBR location between ESDVs (figure 5.17) in tests 5.16 and 5.17 from table 5.3, for the valve spacing $D$ of $1 \mathrm{~km}$ (a) and $5 \mathrm{~km}(b)$.

Figure 5.18 also shows that in contrast with the trend predicted for test 5.16 , for both valve spacings, $M_{\text {total }}$ predicted in test 5.17 (i.e. when using $\mathrm{CV}$ as the downstream isolation valve) progressively decreases with $x / D$. This can be explained by the fact that compared to ASV, the $\mathrm{CV}$ enables faster isolation of the ruptured pipe segment when positioned closer to the rupture plane.

However, the difference in the total inventory loss between tests 5.16 and 5.17 is less than 5\%, meaning that when employed CVs along with ASVs, offers no significant reduction in limiting the amount of inventory loss and the corresponding duration of the accidental release compare to use only ASVs. Consequently, the cost and reliability of emergency isolation systems should be carefully considered when adding CVs to pipelines already equipped with ASVs.

Notably, the $M_{\text {total }}$ data plotted in figure 5.18 for both valve spacings agree closely (within ca. $10 \%$ ) with the initial mass of inventory prior to failure, $M_{o}$ (equation 4.4 ). This means that ASVs with the closing rate of $2.54 \mathrm{~cm} / \mathrm{s}$ (table 5.3) enable relatively fast isolation of the pipeline segment. 
Figure 5.19 shows the total inventory loss, $M_{\text {total }}$ versus FBR location, $x / D$ for test 5.18 (RCVs only; solid lines) and test 5.19 (using RCV combined with CV; dash lines) for emergency isolation (see table 5.3), for the valve spacing distances, $D$ of 1 and $5 \mathrm{~km}$. In contrast with observations in figure 5.18 where $M_{\text {total }}$ was found to relate to the valve spacing, in figure $5.19, M_{\text {total }}$ does not vary significantly with $D$ when use RCVs only (test 5.18 ; less than ca. $10 \%$ difference between $D=1 \mathrm{~km}$ and $5 \mathrm{~km}$ ). This can be explained by the relatively high rates of release, resulting in large amount of fluid escaping from the pipeline prior to the RCV activation (240 s following failure). Remarkably, due to the RCV finite activation time, $M_{\text {total }}$ can still be significant even reducing the valve spacing further.

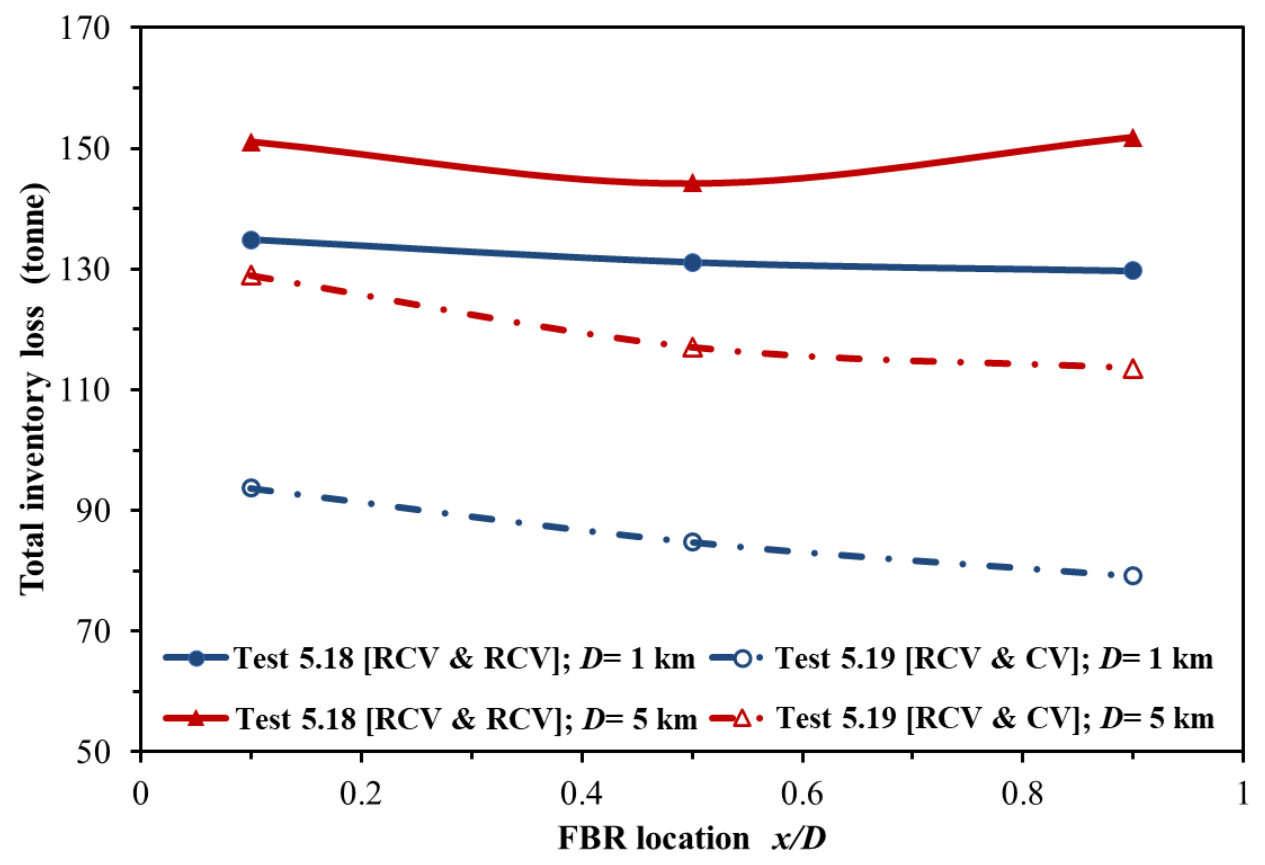

Figure 5.19: Variation of the inventory mass loss as a function of FBR location between ESDVs (figure 5.17) when using RCVs only and RCV combined with CV (table 5.3) for the vale spacing $D$ of $1 \mathrm{~km}$ and $5 \mathrm{~km}$.

Figure 5.19 also shows that combining RCV and CV (test 5.19) offers significant reduction in the total inventory losses as compared to emergency isolation based on RCVs only (test 5.18). Such observation can be explained by significant delay in activation of RCVs as compared with CVs. 
The above observation becomes more pronounced for shorter valve spacing distances $D$, as can be seen from comparison of the difference between the total inventory losses between tests 5.18 and 5.19 predicted for $D=1$ and $5 \mathrm{~km}$. Indeed, as the valve spacing reduces, the total inventory loss from an isolated pipe section depends solely on the $\mathrm{CV}$ activation time that scales with the valve spacing distance, explaining the observed effect. This indicates that in pipelines with relatively short valves spacing, CVs setup in conjunction with RCVs, can help to significantly reduce the amount of total inventory loss prior to complete pipeline isolation, and hence reduce the emergency isolation time for a ruptured section of the pipeline.

\subsection{Concluding Remarks}

This chapter focused on the investigation of the efficacy of ESDV by using different inline valve combinations and spacing on minimising the outflow following pipeline FBR and puncture failures by the application of realistic test cases. Two typical high-pressure onshore pipelines were used as case studies with one transporting ethylene whist the other transporting $\mathrm{CO}_{2}$. Given that ethylene is highly flammable, explosive and potentially toxic at high concentrations, and $\mathrm{CO}_{2}$ is an asphyxiant at concentrations above $7 \%(\mathrm{v} / \mathrm{v})$, the assessment of such pipelines in the event of pipeline failure is of paramount importance.

The results of the investigation presented in this chapter showcased particularly important in demonstrating the usefulness of the transient outflow model as a valuable investigative tool for studying the efficacy of different ESDV configuration varying its type, combination, number, spacing and closure characteristics on limiting the outflow following the failure of pressurised pipelines. These information provide imperative guidelines for emergency planning and mitigation measures.

Based on the results and analysis presented, the following key conclusions of practical significance may be made:

- The impact of RCV and CV spacing in minimising outflow following a puncture failure at mid-way along a hypothetical $\mathrm{CO}_{2}$ pipeline was investigated. It was found that the installation of 2 equidistant CVs progressively resulted in a reduction of the amount of cumulative mass discharged by ca. $50 \%$. However, further increase in the number of 
$\mathrm{CVs}$ beyond 2 had little effect on further reduction in the amount of cumulative mass discharged

- Switching to RCV placed at both upstream and downstream of the puncture, no such limit was observed. The amount of cumulative mass discharged simply reduced with an increase in the number of valves. However, such a reduction obtained by increasing the number of valves became marginal above certain range due to the long valve activation time resulting in significant amount of inventory loss prior to valve activation

- In the case of using both RCVs and CVs at the same location (i.e. in tandem), significant improvement was observed in minimising outflow compared to only using one type of valve. For example, with $2 \mathrm{CVs}$ and $2 \mathrm{RCVs}$, the corresponding reduction in the amount of cumulative mass discharged was significantly higher than using 8 same type valves placed along the punctured pipeline. The above demonstrated the potential merits in the choice of using different type of valves at the same locations for ESDV optimisation

- The efficacies of ASVs, RCVs and CVs and their combinations were also assessed by comparing the amount of total inventory loss from an accidental FBR in a section between two ESDVs setup along a hypothetical ethylene pipeline. It was found that for different FBR locations, with two ASVs installed along the pipeline, the time of activation of the valves, and the resulting total inventory mass losses were mainly affected by the distance between the valve and the rupture plane, but not the initial feed flow prior to failure. The results also showed that amount of total inventory loss agreed closely (within ca. 10\%) with initial mass of inventory prior to failure. This indicated that using ASVs enabled relatively fast isolation of the pipeline segment following FBR

- Compared to using ASVs only, the combination of ASV and CV showed no significantly impact on the reduction in limiting the amount of total inventory loss and hence the duration of release following FBR. As such, the cost and reliability of emergency isolation systems should be carefully considered when adding CVs to pipelines already equipped with ASVs

- On the other hand, switching to RCVs placed at both upstream and downstream of the FBR, the amount of total inventory loss was significantly higher as compared to using ASVs. Also, the amount of total inventory loss did not vary significantly with the valve 
spacing. This finding again indicated that due to the finite response time of RCVs, the duration of accidental release prior to complete isolation cannot be reduced below a threshold using shorter valve spacing

- In the case of combining RCV and CV, significant reduction in the total inventory loss as compared to emergency isolation based on RCVs only was observed. As such, installing additional CVs in high-pressure ethylene pipelines equipped with RCVs, could significantly reduce the duration of release, hence assisting faster accident consequence mitigation

Given the large number of design and control parameters involved, the next chapter is devoted to developing and applying a multi-objective optimisation technique along with principal component analysis to determine the optimal ESDV configuration in high-pressure pipelines for striking the balance among three objective functions: the amount of inventory escaping prior to complete valve closure following FBR, the ESDV capital cost and the total failure rate for all ESDVs employed along the pipeline. 
Chapter 6:

Optimising Emergency Shutdown Valve Configurations for Pressurised Pipelines Using Multi-objective Optimisation and Principal Component Analysis

\subsection{Introduction}

So far, the main focus of this work has been studying Emergency Shutdown Valve (ESDV) characteristics and their efficacy in isolating outflow following accidental failures of highpressure pipelines. Another important consideration in practice is the significant capital cost of ESDVs, which can become prohibitive depending on their type (ASVs or RCVs are much more expensive than CVs) (Smith and Zappe, 2004) and number of units employed. As such, the optimum configuration of ESDVs requires a delicate balance between their total cost and the level of protection these provide in minimising the resulting risks in the event of a pipeline failure. Such challenge may be defined as a multi-objective problem.

As mentioned in Chapter 3, multi-objective optimisation methods are often used in practice for dealing with reliability-based and risk-informed assessments for system design, operation, maintenance and regulation (Zio and Bazzo, 2011; Solomon Brown et al., 2014; Demissie, 2015; HSE, 2017).

Brown et al. (2014) for example presented a study using the above method to optimise valve spacing in $\mathrm{CO}_{2}$ pipelines as a trade-off between the reduction in risk against the cost in ESDV installation and maintenance. However, with the exception of the valve spacing, the impact of other important parameters such as ESDV type \& their combination, number and operational settings (e.g. activation \& closure times) were not considered.

Unfortunately, increasing the number of optimisation variables leads to significant additional mathematical complexities, making solutions difficult to visualise and analyse. Variable reduction techniques, such as Principal Component Analysis (PCA), attempt to overcome this limitation by identifying redundant variables that can be disregarded whilst still ensuring an improved outcome (Krzanowski, 2000; Gutiérrez et al., 2010; Sabio et al., 2012). 
Therefore, in this chapter, for the first time, we integrate the multi-objective optimisation method with PCA in order to find the optimal ESDV configurations for high-pressure pipelines balancing the ESDV capital cost, the ESDV efficacy in minimising and ultimately isolating outflow following pipeline failures as well as the total failure rate for different combinations of inline ESDVs. Although important, for the sake of simplicity, and lack of reliable data, costs of valve maintenance are not considered in this study.

Natural gas is chosen as the transported fluid given its hazardous nature (extremely flammable and explosive) and its extensive potential use as a fossil fuel worldwide. Given that natural gas is considered as a cleaner transition energy as compared to other energy types (i.e. coal and petroleum), it has emerged as a widely accepted pathway for reducing $\mathrm{CO}_{2}$ emissions in many countries (Demirbas, 2002; Semin and Bakar, 2008; Dong et al., 2020). In China for example, the consumption of natural gas has increased significantly in the last two decades; from 25.3 billion cubic meters (bcm) in 2000 to $307.3 \mathrm{bcm}$ in 2019, (ca. 12 fold increase) (China Statistics Press, 2019). Fuelling this fast-rising demand for natural gas in China is the country's rapidly expanding natural gas infrastructure. According to the 13th Five Year Plan of China (State Council of the People's Republic of China, 2016), a total length of ca. $163000 \mathrm{~km}$ natural gas pipeline network is expected to be constructed by the end of 2025, corresponding to ca. $55 \%$ increase as compared to 2020 . Given that these pipelines may by necessity pass through densely populated urban areas in order to maintain the economic viability, their safe operation is of paramount importance.

As such, in order to ensure practical relevance, a real $1016 \mathrm{~mm}$ i.d., $150.2 \mathrm{~km}$ long pipeline designed by China Petroleum Pipeline Engineering Corporation (CPPEC) (2012) transporting high-pressure natural gas is adopted as a case study in this chapter as follows:

Section 6.2 commences with the description of multi-objective optimisation theory. This is followed by the presentation of the basic mathematical formulation of PCA, including the Yanai's Generalized Coefficient of Determination (GCD) criterion and the Restricted Improvement algorithm. In Section 6.3, the case study for determining the optimal ESDV configuration for the CPPEC natural gas pipeline in China is defined.

Starting with defining a set of 6 optimisation variables for ESDV settings, PCA is first employed to reduce the number of optimisation variables, whilst retaining good agreement for the problem solution and emphasis on computational simplification. The outflow simulation 
results obtained using the outflow model described in Chapter 2 are used as the input conditions for PCA.

Next, a description of the optimisation problem is performed, detailing the objective functions used, including valve capital cost, and the various parameter realistic values assumed. The results of the multi-objective optimisation are presented and discussed using scatter plots providing a geometrical visualisation of the Pareto Front and Set. Conclusions are presented in the last section.

\subsection{Theory}

\subsubsection{Multi-objective Optimisation}

A multi-objective problem can be stated in the form (Zio and Bazzo, 2011):

$$
\min _{\theta \in D} J(\theta)
$$

where,

$$
\theta=\left[\theta_{1}, \ldots, \theta_{p}\right] \in D
$$

$$
J(\theta)=\left[J_{1}(\theta), \ldots, J_{s}(\theta)\right]
$$

Here, $J$ is a vector of objective functions which constitute the multi-objective problem. $\theta$ is the vector of independent variables which are mapped from $D$, the decision space to the vector space spanned by $J$.

Assuming a multi-objective problem with, $s$ objectives is to be considered, where, $l$ objectives are to be minimised and, $m$ objectives are to be maximised. Each objective can be normalised with respect to its minimum or maximum values on the Pareto Front (Zio and Bazzo, 2011): 
$\begin{array}{ll}\bar{J}_{l}(\theta)=\frac{J_{i}(\theta)-J_{i}^{\text {min }}}{J_{i}^{\text {max }}-J_{i}^{\text {min }}} & i=1, \ldots, l \\ \bar{J}_{l}(\theta)=\frac{J_{i}^{\text {max }}-J_{i}(\theta)}{J_{i}^{\text {max }}-J_{i}^{\text {min }}} & i=l+1, \ldots, s\end{array}$

and

$0 \leq \overline{J_{l}}(\theta) \leq 1$

$i=1, \ldots, s$

where, $\overline{J_{l}}(\theta)=0$ means that solution, $\theta$ has the best value for the $i$ th objective. And $\overline{J_{l}}(\theta)=1$ means that solution, $\theta$ has the worst value for the $i$ th objective.

\subsubsection{Principal Component Analysis (PCA)}

Mathematically, PCA is defined as an orthogonal linear transformation that converts the interrelated variables, $\theta_{1}, \theta_{2}, \ldots, \theta_{p}$ into a smaller set of uncorrelated variables, known as Principal Components (PCs), $y_{1}, y_{2}, \ldots, y_{p}$ in a new coordinate system, such that:

$$
y=\Gamma^{T} \theta
$$

where, $\theta$ and $y$ are $p$-dimentional random vectors. $\Gamma$ is the $p \times p$ orthogonal data matrix. The covariance matrix, $\Sigma$ of the variables, $\theta$ as well as the eigenvalues and corresponding eigenvectors of $\Sigma$ are calculated using equation 6.7. PCs are ordered according to the amount of variance they pertain to. The greatest variance by any projection of the inter-related variables comes to lie on the first coordinate (called the first PC), the second greatest variance on the second coordinate, and so on.

According to Jolliffe (1972), a dimension is considered to be important if the eigenvalue of the $\mathrm{PC}$ is greater than 0.8 , although the usual approach is to consider a dimension as important if the eigenvalue is greater than 1 . However, dimensionality reduction via PCA does not provide 
a real reduction of dimensionality in terms of the original variables, since all of the original variables are required to define even a single PC. Also, the PCs do not reliably indicate which variables are the most relevant in terms of preserving information.

In order to select a subset of $k$ PCs from, $p$ while still retaining most of the variance existing in the full-space problem, a common approach is to account for more than $90 \%$ of the total variance (Cadima and Jolliffe, 2001; Gutiérrez et al., 2010). The proportion of the total variation explained by the first $k$ PCs, $P r_{k}$ can be given by (Gutiérrez et al., 2010):

$$
\operatorname{Pr}_{k}^{2}=\frac{\sum_{j=1}^{k} \lambda_{j}}{\sum_{j=1}^{p} \lambda_{j}}=\frac{\sum_{j=1}^{k} \lambda_{j}}{\operatorname{tr}(\Sigma)}
$$

where, $\lambda_{j}$ is the variance of the $j$ th $\mathrm{PC}$, which is equal to the $j$ th largest eigenvalue of the covariance matrix, $\Sigma$ of the variables, $\theta$ such that:

$$
\lambda_{j}=\operatorname{Var}\left(y_{j}\right)
$$

Given that for $p$ variables, there are $2^{p}-1$ possible subsets for the complete search since each potential independent variable can either be included or excluded (Gutiérrez et al., 2010), the above variable selection method quickly becomes infeasible and computational expensive with the increasing number of variables. For example, for 10 variables, the number of all possible subsets is 2021 , while for 20 variables, the above number increases rapidly to more than 1 million. In order to address this problem, several methods have been proposed to perform the variable selection in multivariate analysis such as the Yanai's generalized coefficient of determination (GCD) criterion (Ramsay et al., 1984) presented as follows.

\subsubsection{The Yanai's Generalized Coefficient of Determination (GCD) criterion}

The GCD is defined as the cosine of the angle between the matrices of orthogonal projections on two subspaces, in which the degree of similarity between those subspaces is measured. Recent studies using the GCD criterion in the context of PCA have shown good performance in the selection of best subset of variables and significant improvement in the computational 
simplification (Cadima and Jolliffe, 2001; Cadima et al., 2004; Gutiérrez et al., 2010). As such, the GCD criterion is employed in this study.

The GCD is defined by a $n \times p$ data matrix, $\mathbf{X}$, whose columns result from regressing each of the $p$ (centred) observed variables on $K$ (i.e., orthogonally projecting them on $\mathbf{K}$ ) such that:

$$
\mathrm{GCD}=\cos \left(\mathbf{P}_{\mathbf{G}}, \mathbf{P}_{\mathbf{K}}\right)=\frac{1}{k} \operatorname{tr}\left(\left[\mathbf{S}_{\{G\}}\right]_{(K)}\left[\mathbf{S}_{\{K\}}\right]^{-1}\right)
$$

where, $\mathbf{P}_{\mathbf{K}}$ and $\mathbf{S}_{\{K\}}$ are the matrix of the orthogonal projections on $\mathbf{K}$ and constitute the submatrix which results from retaining those rows and columns of $\mathbf{S}$, whose row/column numbers are found in $K$, respectively. The subspace, $\mathbf{G}$, spanned by the first $k$ PCs of the data set is defined as the maximum possible variability within the original data set. $\mathbf{P}_{\mathbf{G}}$ and $\mathbf{S}_{\{G\}}$ are the matrix of orthogonal projection onto subspace $\mathbf{G}$ and the approximation to matrix $\mathbf{S}$ obtained by retaining, respectively. It should be noted that only the eigenvectors values associated with the $k$ largest eigenvalues are used in the spectral decomposition of $\mathbf{S}$. $\left[\mathbf{S}_{\{G\}}\right]_{(K)}$ is the $k \times k$ submatrix of $\mathbf{S}_{\{G\}}$ obtained by retaining only the rows/columns with row/column numbers in $K$.

Rewriting the GCD criterion in a simpler algebraic form (Cadima and Jolliffe, 2001), we get:

$$
\mathrm{GCD}=\frac{1}{k} \sum_{i=1}^{k}\left(r_{m}\right)_{i}^{2}
$$

where, $\left(r_{m}\right)_{i}$ is the multiple correlations between the $i$ th PC of the full data set and the $k$ selected variables.

The GCD is the average of the squared canonical correlations between two sets of variables spanning each of the subspaces, with a value between 0 (if the subspaces are orthogonal) and 1 (if all $k$ PCs are in $\mathbf{K}$, i.e., if the subspaces coincide). 


\subsubsection{The algorithm: The Restricted Improvement (Cadima et al., 2004)}

Finding the set, $S$ which maximise equation 6.10 for a given value of $k$ can be defined as a general combinatorial optimisation problem as follows:

find $S$ which maximises $\left\{c(S): S \in \mathcal{F} \subseteq 2^{N}\right\}$

where, $N$ is a finite set, and the objective function $c: 2^{N} \rightarrow \mathbb{R}_{0}^{+}$associates to each subject of $N$ a nonnegative real value. The set $S^{*} \subseteq N$ is an optimal solution if $S^{*}$ is in the set of $\mathcal{F}$ of feasible solutions and $c\left(S^{*}\right) \geq c(S)$, for all $S \in \mathcal{F}$.

Local search heuristics are often used throughout for the calculations inside the feasible region $\mathcal{F}$, by exchanging some of its components to move from one feasible solution to another feasible solution (Martin et al., 1992; Arya et al., 2004; Cadima et al., 2004). Different heuristic algorithms can be applied for combinatorial optimisation problems to find the best subset of variables for the successive $k$-values. In this study, the Restricted Improvement technique proposed by Cadima et al. (2004) is employed to determine the set, $S$ for this purpose.

Figure 6.1 shows the corresponding calculation flow algorithm. Here, the set $X$, which contains $k$-subsets, is defined as the neighbourhood of the current solution, $S$. The set, $X$ on the other hand can be obtained by replacing one element of, $S$ with one element from $X \backslash S$. The set, $Q$ which is defined as the queue to arrange the variables in the set $X$, consists of all variables which are not included in the starting solution initially.

As can be observed in figure 6.1, the algorithm commences with picking and removing an element, $j$ from the set, $Q$. Then, the variable, $i^{*}$ is determined to satisfy the following correlation: find $i^{*}\left(i^{*} \in S\right)$ which maximises $\{c(S \backslash\{i\} \cup\{j\}): i \in S\}$. Next, the current set, $S$ is updated by defining, $S=S \backslash\left\{i^{*}\right\} \cup\{j\}$ (if and only if $c\left(S \backslash\left\{i^{*}\right\} \cup\{j\}\right)>c(S)$ ). Thereafter, the variable, $i^{*}$ is inserted into the set, $Q$, if and only if, $i^{*}$ has not been in $Q$ before. Finally, the algorithm stops until the set $Q$ is equal to the empty set (if the set $Q$ is not equal to the empty set, go back to the first step and repeat the process). It should be noted that the whole process gives $O(k \times p)$ evaluations of the criterion given that determining, $i^{*}$ involves $k$ evaluations of the objective function and no element is inserted into the set $Q$ twice. 


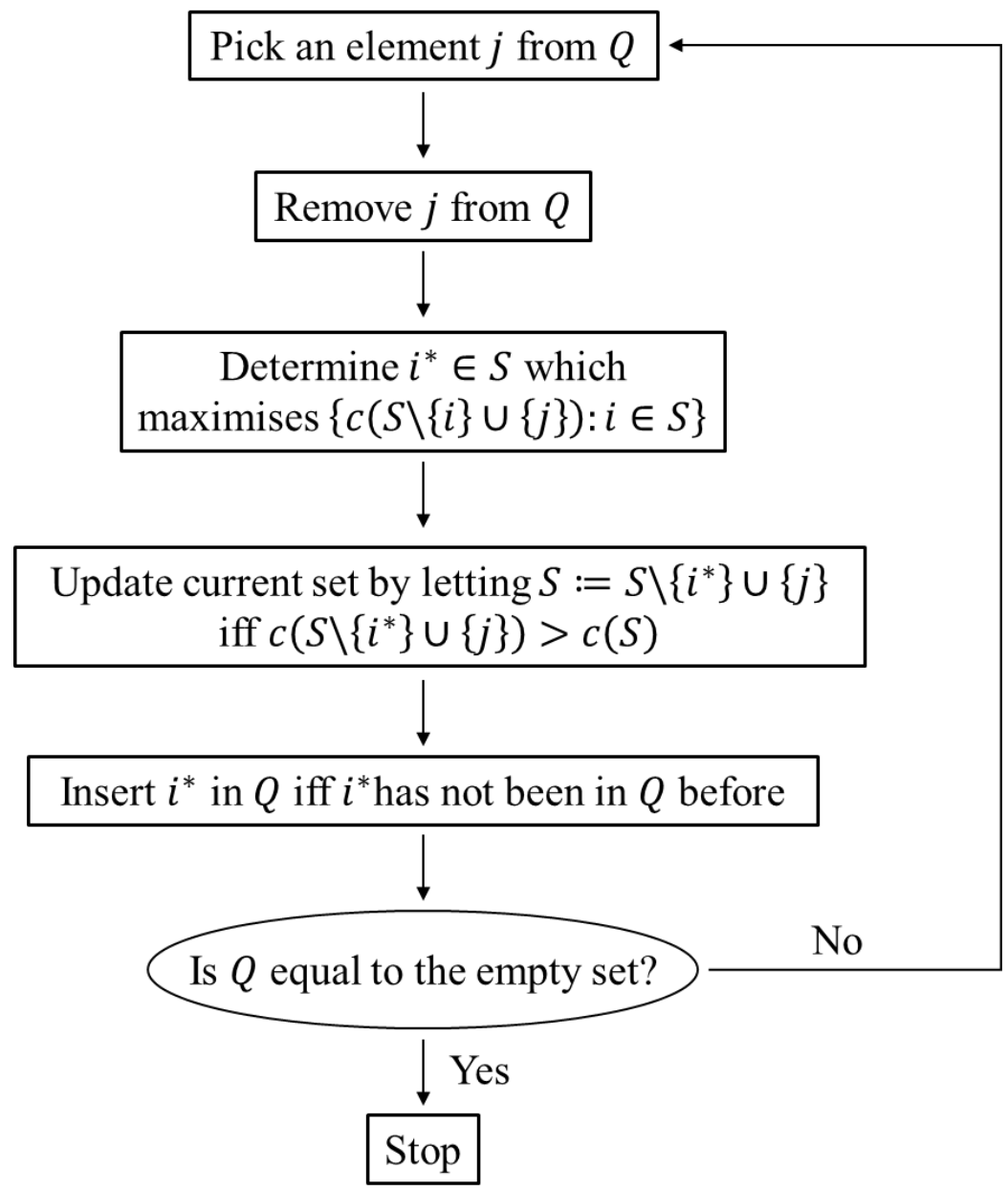

Figure 6.1: The calculation flow algorithm of the Restricted Improvement.

\subsection{Results and Discussion}

\subsubsection{Case Study}

For the purposes of this study, the relevant conditions for the planned high-pressure natural gas pipeline to be constructed by CPPEC (2012) are adopted for the ESDV configuration multiobjective optimisation investigation. The pipeline is designed for the Harbin - Shenyang natural gas transmission pipeline project, with a total length of $555 \mathrm{~km}$, expected to transport 5.9 million tonnes per annum natural gas from the end of 2020. Figure 6.2 gives a schematic representation of the route of the Harbin - Shenyang gas transmission pipeline project marked 
in red. As it may be observed, the natural gas pipeline starts from Harbin, running through several populous cities including Dehui, Changchun, Siping and Kaiyuan, and eventually terminates in Shenyang. Given its proximity to these highly populated areas, its safe operation is of paramount importance.

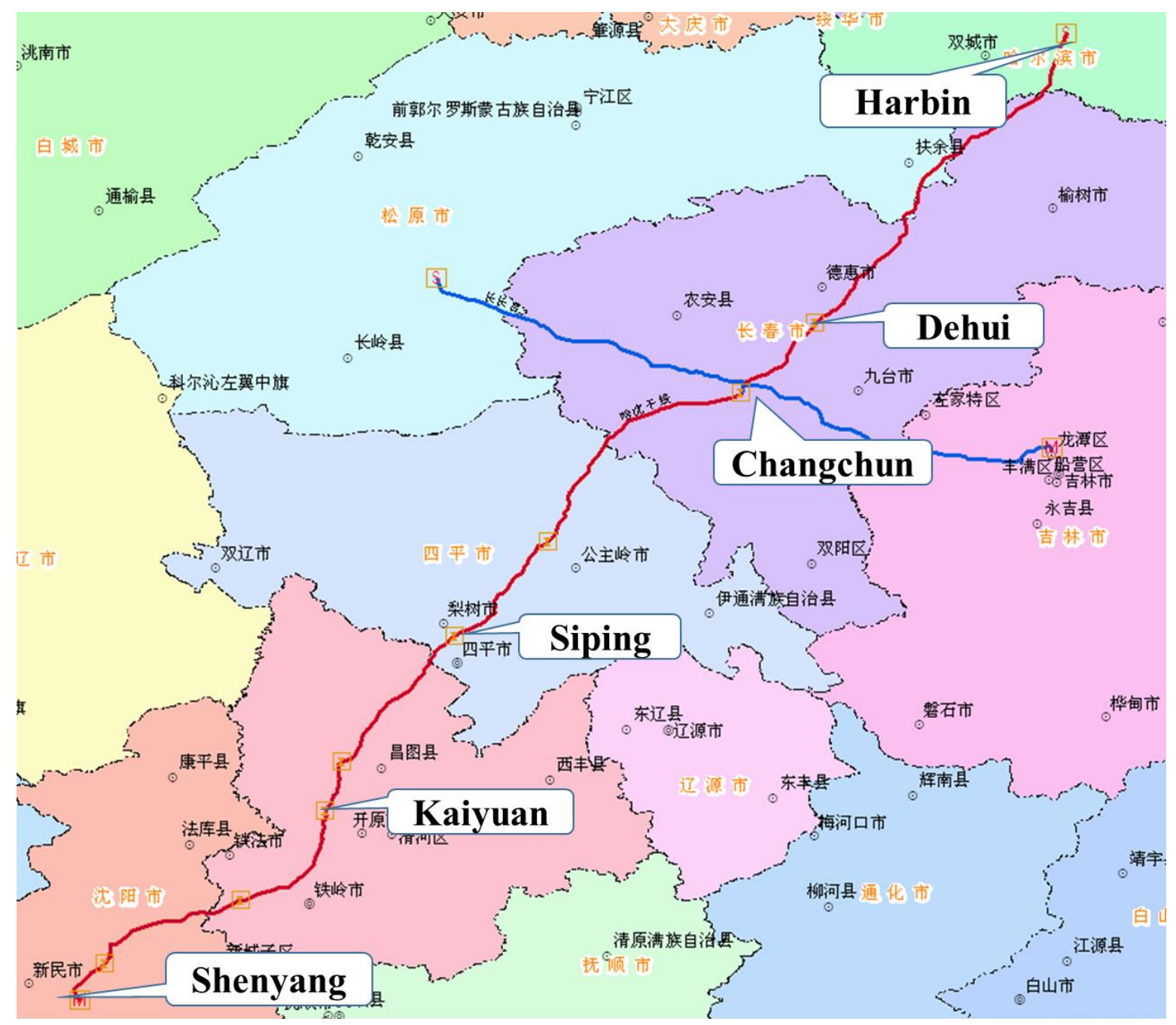

Figure 6.2: Schematic representation of the route (indicated in red) of the Harbin - Shenyang natural gas transmission pipeline project (CPPEC, 2012).

This case study focuses on the first part of the natural gas pipeline; from Harbin to Dehui, spanning a total length of $150.2 \mathrm{~km}$. The operating feed flow rate is $211.58 \mathrm{~kg} / \mathrm{s}$ and the line pressure and temperature are at 80 bar and $307.24 \mathrm{~K}$ respectively. Other relevant details regarding the pipeline setup, including the fluid composition are presented in table 6.1. 
Table 6.1: Pipeline setup for the case study.

\begin{tabular}{|c|c|c|c|c|c|c|c|c|}
\hline \multicolumn{5}{|c|}{ Pipeline Parameters } & \multicolumn{4}{|c|}{ Value } \\
\hline \multirow{4}{*}{ Pipeline } & \multicolumn{4}{|c|}{ Pipeline outer diameter (mm) } & \multicolumn{4}{|l|}{1051} \\
\hline & \multicolumn{4}{|c|}{ Pipeline wall thickness (mm) } & \multicolumn{4}{|l|}{17.5} \\
\hline & \multicolumn{4}{|c|}{ Pipeline roughness height (mm) } & \multicolumn{4}{|c|}{0.005 (assumed for mild steel) } \\
\hline & \multicolumn{4}{|c|}{ Heat transfer coefficient $\left(\mathrm{kW} / \mathrm{m}^{2} \mathrm{~K}\right)$} & \multicolumn{4}{|c|}{5 (assumed) } \\
\hline \multirow{2}{*}{$\begin{array}{l}\text { Ambient } \\
\text { conditions }\end{array}$} & \multicolumn{4}{|c|}{ Ambient pressure (bar) } & \multicolumn{4}{|l|}{1} \\
\hline & \multicolumn{4}{|c|}{ Ambient temperature $(\mathrm{K})$} & \multicolumn{4}{|c|}{293.15 (assumed) } \\
\hline \multirow{3}{*}{$\begin{array}{l}\text { Boundary } \\
\text { conditions }\end{array}$} & \multicolumn{4}{|c|}{ Upstream boundary condition } & \multicolumn{4}{|l|}{ Pump } \\
\hline & \multicolumn{4}{|c|}{ Pump Shutdown time } & \multicolumn{4}{|c|}{$\begin{array}{l}60 \text { seconds after leak detected } \\
\text { (assumed) }\end{array}$} \\
\hline & \multicolumn{4}{|c|}{ Downstream boundary condition } & \multicolumn{4}{|l|}{ Closed } \\
\hline Feed & $\mathrm{CH}_{4}$ & $\mathrm{C}_{2} \mathrm{H}_{6}$ & $\mathrm{C}_{3} \mathrm{H}_{8}$ & $\mathrm{i}-\mathrm{C}_{2} \mathrm{H}_{10}$ & $n-C_{4} H_{10}$ & $\mathrm{CN}_{2}$ & $\mathrm{CO}_{2}$ & $\mathrm{He}$ \\
\hline $\begin{array}{c}\text { Mole fraction } \\
\text { (\%) }\end{array}$ & 94.7 & 0.55 & 0.08 & 0.01 & 0.01 & 1.92 & 2.71 & 0.02 \\
\hline
\end{tabular}

In this study, pipeline locations risk ratings are classified based on proximity to occupied buildings or other areas where people gather regularly. Accordingly, ESDVs are proposed to be installed at specified intervals in consonance with the 3 escalating Risk Classifications Ratings (RCR) defined by the CPPEC (2012) as presented in table 6.2. The corresponding criteria for the maximum ESDV spacing under each RCR are also included in the same table. Figure 6.3 shows the RCR distribution along the Harbin-Dehui natural gas pipeline. 
Table 6.2: Risk Classifications Ratings and maximum ESDV spacing for the Harbin Shenyang natural gas transmission pipeline (CPPEC, 2012).

\begin{tabular}{|l|c|c|}
\hline $\begin{array}{l}\text { Risk Classification } \\
\text { Rating (RCR) }\end{array}$ & $\begin{array}{l}\text { Numbers of households living within } \\
\text { 2km radius of the pipeline }\end{array}$ & $\begin{array}{l}\text { Maximum ESDV } \\
\text { spacing (km) }\end{array}$ \\
\hline Class 1 & $\leq 15$ & 32 \\
\hline Class 2 & $>15, \leq 100$ & 24 \\
\hline Class 3 & $>100$ & 16 \\
\hline
\end{tabular}

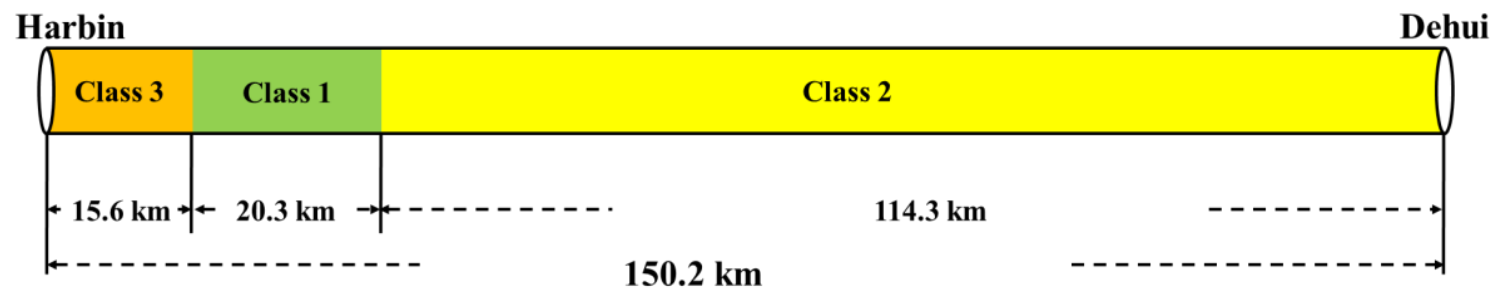

Figure 6.3: Schematic representation of Risk Classification Rating (RCR) distribution along the Harbin-Dehui natural gas pipeline (CPPEC, 2012).

As can be seen from figure 6.3, the Harbin-Dehui natural gas pipeline is classified into 3 RCRs along the route: Class $3(15.6 \mathrm{~km})$, Class $1(20.3 \mathrm{~km})$ and Class $2(114.3 \mathrm{~km})$. In order to satisfy the criteria for the maximum ESDV spacing under the given RCR along the Harbin-Dehui natural gas pipeline as indicated in table 6.2 and figure 6.3, a minimum of 6 ESDVs are required to install along the pipeline. Figure 6.4 shows the schematic representation of the proposed valve spacing for 6 ESDVs (denoted as ESDV-1 to ESDV-6) along the Harbin-Dehui natural gas pipeline. The detailed ESDV location along the pipeline, spacing and RCR between two ESDVs as well as the maximum ESDV spacing are presented in table 6.3. 


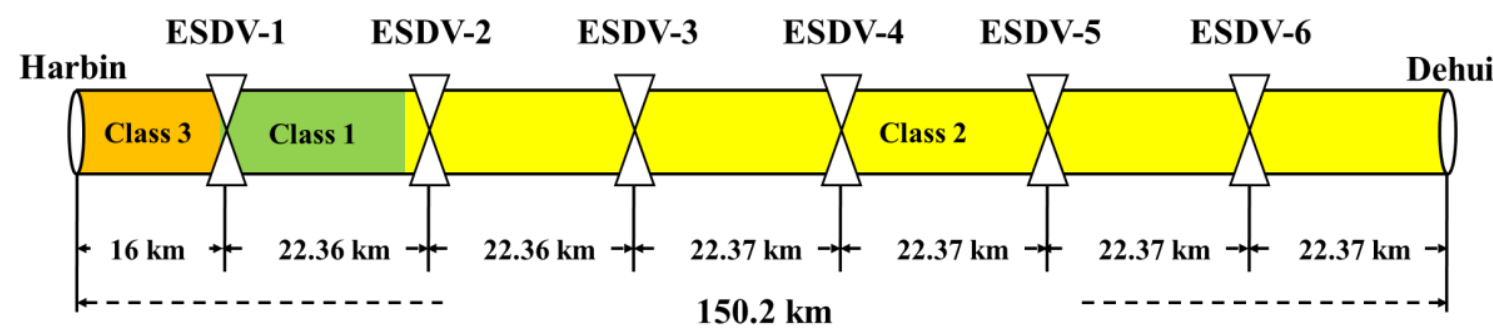

Figure 6.4: Schematic representation of the proposed valve spacing for 6 ESDVs along the Harbin-Dehui natural gas pipeline.

Table 6.3: Proposed valve spacing for 6 ESDVs installed along the Harbin-Dehui natural gas pipeline.

\begin{tabular}{|l|c|c|c|c|}
\hline & $\begin{array}{l}\text { ESDV Location } \\
\text { along the pipeline } \\
(\mathbf{k m})\end{array}$ & $\begin{array}{l}\text { Spacing } \\
\text { between } \\
\text { ESDVs (km) }\end{array}$ & $\begin{array}{l}\text { Risk classification } \\
\text { rating } \\
\text { between 2 ESDVs }\end{array}$ & $\begin{array}{l}\text { Maximum } \\
\text { ESDV spacing } \\
\text { (km) }\end{array}$ \\
\hline Harbin & 0 & 0 & Class 3 & 16 \\
\hline ESDV-1 & 16 & 16 & Class 3 \& 1 & 16 \\
\hline ESDV-1 & 38.36 & 22.36 & Class 1 \& 2 & 24 \\
\hline ESDV-1 & 60.72 & 22.36 & Class 2 & 24 \\
\hline ESDV-1 & 83.09 & 22.37 & Class 2 & 24 \\
\hline ESDV-1 & 105.46 & 22.37 & Class 2 & 24 \\
\hline ESDV-1 & 127.83 & 22.37 & Class 2 & 24 \\
\hline Dehui & 150.2 & 22.37 & Class 2 & 24 \\
\hline
\end{tabular}

For the purpose of optimising ESDV configurations for the above pipeline, 6 optimisation variables for the various ESDV settings are considered for the multi-objective optimisation study. The objectives being:

1. minimising the amount of total inventory loss prior to complete valve closure following FBR

2. minimising the ESDV capital cost 
3. minimising the total failure rate for all ESDVs used along the pipeline

The corresponding optimisation variables include:
i. ESDV type (ASV and RCV)
ii. ESDV activation pressure
iii. ESDV combination
iv. number of ESDVs
v. ESDV spacing
vi. FBR location

Table 6.4 presents the corresponding simulation input parameters of the above optimisation variables. Here the RCVs are assumed to close 60s following the leak detection. The ASVs on the other hand are assumed to close immediately once the leak is detected. For both RCVs and ASVs, the valve linear closure rate is assumed to be $1.904 \mathrm{~cm} / \mathrm{s}$. It should be noted that CVs are not suitable to be used solely for emergency isolation in long multi-segment pipelines (CVs are designed to prevent backflow, they will not be activated if a pipeline failure occurs downstream of the installed CVs; as discussed in Section 4.3.2, Chapter 4 and Section 5.2.2, Chapter 5). Therefore, only RCVs and ASVs are assumed to be installed along the pipeline.

Table 6.4: Simulation input parameters of the selected 6 optimisation variables for ESDV configuration.

\begin{tabular}{|l|l|}
\hline $\begin{array}{l}\text { Optimisation Variables Related with } \\
\text { ESDV Settings }\end{array}$ & Simulation Input Parameters \\
\hline ESDV type & RCV and ASV \\
\hline ESDV activation pressure & $2,5,10,20$ and 30 bar \\
\hline ESDV combination & RCV only; ASV only; RCV \& ASV \\
\hline Number of ESDVs & $\geq 6$ \\
\hline ESDV spacing & $\leq 16 \mathrm{~km}($ Class 3$) ; \leq 24 \mathrm{~km}(\mathrm{Class} 2)$ \\
\hline
\end{tabular}


FBR location $x / D=0.1 ; 0.3 ; 0.5 ; 0.7 ; 0.9$ (where $x$ is the distance between FBR and the nearest ESDV downstream of FBR. $D$ is the distance between two ESDVs)

In this case study, given the large number of parameters, PCA is first applied to discard the redundant optimisation variables for the multi-objective optimisation study. The outflow calculations are carried out using the Homogeneous Equilibrium Mixture (HEM) model described in Chapter 2 and the simulations results are in turn used as the input conditions PCA. For each optimisation variable listed in table 6.4, 30 tests are performed varying the input parameters which are selected by using a random value generator. It should be noted here that the outflow data fed into PCA is normalised in order to reduce the computational complexity and simulation runtime.

Following the results of PCA, the multi-objective optimisation study is conducted. The multiobjective optimisation problem can be summarised in the following mathematical form:

$$
\min _{\theta \in D} J_{1}(\theta) J_{2}(\theta) J_{3}(\theta) \quad \theta \in I
$$

Here, $\theta$ is the vector of the independent optimisation variables obtained from the results of PCA. $I$, is the interval which the optimisation variables lie within (see table 6.4).

$J_{1}(\theta)$ is the metric for the hazard associated with a pipeline failure. In this study, the objective function $J_{1}$, is defined to be the total inventory loss prior to complete valve closure following FBR failure.

$J_{2}(\theta)$, the annual cost of the set of $N$ ESDVs, is expressed by (Medina et al., 2012):

$$
J_{2}(\theta)=\frac{V_{P N} r(1+r)^{n}}{(1+r)^{n+1}-1} N
$$

where, $V_{P N}$ is the single valve cost. $r$ and $n$ are the discount rate and the average lifetime of the equipment, respectively. The values used here are $V_{P N}(A S V)=£ 140000, V_{P N}(R C V)=£$ $60000, r=0.035$ and $n=10$ years (CPPEC, 2012). It is worth mentioning that $J_{2}(\theta)$ should be 
less than the maximum budget for the annual cost of the ESDVs, which is assumed to be $£ 100000$ in this case study.

$J_{3}(\theta)$ is the total failure rate for all ESDVs used along the pipeline. According to Faraci (2006), it can be assumed that the total failure rate is the sum of the individual ESDV failure rate. The individual failure rate for RCV and ASV used here are assumed to be 0.03 /demand and 0.01 /demand respectively (HSE, 2017).

In this study, the scatter plot is introduced to provide a geometrical visualisation of the Pareto Front and Set as it is one of the most common straightforward visualisation techniques to project all individual objective information of the underlying approximation set. The plots are obtained by plotting each variable on the coordinate system set up by the 3 objectives, $J_{1}, J_{2}$ and $J_{3}$. The plots can then be used to select an optimal set of values for the optimisation variables.

\subsubsection{Principal Component Analysis (PCA) Results}

PCA is first applied as the variable reduction technique to discard the redundant optimisation variables for the multi-objective optimisation study without disturbing the main features of the whole data set. The results of PCA are summarised in table 6.5. The eigenvalues of the 6 PCs demonstrate the variability in each associated PC. The proportion and cumulative variabilities of each PC as well as the eigenvectors of each optimisation variable are also given in the table. It is worth noting that the proportional variability for each PC is measured by the ratio of the eigenvalues to the sum of the eigenvalues. 
Table 6.5: Summary of PCA results using the $6 \times 6$ matrix.

\begin{tabular}{|l|cccccc|}
\hline \multicolumn{1}{|l}{ Principal component no. } & $\mathbf{\# 1}$ & $\mathbf{\# 2}$ & $\mathbf{\# 3}$ & $\mathbf{\# 4}$ & $\mathbf{\# 5}$ & $\mathbf{\# 6}$ \\
\hline Eigenvalues & 4.026 & 1.934 & 0.816 & 0.296 & 0.221 & 0.001 \\
Proportion variability & 0.5632 & 0.2695 & 0.0999 & 0.0399 & 0.0273 & 0.0001 \\
Cumulative variability & 0.5632 & 0.8327 & 0.9326 & 0.9725 & 0.9998 & 1.0000 \\
& & & & & -0.113 & 0.016 \\
ESDV type & 0.947 & -0.285 & 0.034 & 0.011 & -0.143 & 0.009 \\
ESDV activation pressure & 0.933 & -0.302 & -0.044 & -0.087 & -0.118 & -0.025 \\
ESDV combination & 0.673 & -0.187 & -0.027 & -0.047 & 0.277 & 0.002 \\
Number of ESDVs & 0.926 & 0.499 & -0.214 & -0.318 & 0.273 \\
ESDV spacing & 0.534 & 0.679 & -0.281 & 0.417 & -0.013 & 0.001 \\
FBR location & 0.401 & -0.836 & 0.053 & 0.230 & 0.318 & -0.001 \\
\hline
\end{tabular}


According to Jolliffe (1972), a dimension is important if the eigenvalue is greater than 1. As can be observed from table 6.5, only for the first two PCs, their eigenvalues are greater than 1 . Therefore, the data set of the 6 optimisation variables are essential in two-dimensional analysis.

According to table 6.5, the eigenvalues for the first and second PCs are respectively 4.026 and 1.934. Their combination accounts for 0.8327 or $83.27 \%$ of the total variability. The first PC accounts for $56.32 \%$ of the proportion variability and appears to be primarily driven by three optimisation variables: the ESDV type, ESDV activation pressure and number of ESDVs. The second PC accounts for over $26 \%$ of the proportion variability. It is noteworthy that for the second PC, the signs of the eigenvectors for the optimisation variables have no importance in themselves as all may be reversed without undermining the analysis. However, the signs can indicate a classification of the optimisation variables into two groups: the first group including the number of ESDVs and ESDV spacing, and all the other optimisation variables are in the second group. The sign of the eigenvectors can also suggest that the second PC directs large values of the second group and low values of the first group in two different directions of the subspace.

In order to select the most promising subsets of optimisation variables from different numbers of indicators (denoted as $k$ ), both the GCD criterion and the total variance associated with the first $k$ PCs are used. Table 6.6 shows, for the 6 the optimisation variables, the results of the total variance and the GCD for $k$ PCs. The corresponding indicators for the optimal subsets of optimisation variables associated with each $k$ value are also presented in the table. The total variance of $k$ PCs relates to the percentage of the cumulative variability (as in table 6.5). The Restricted Improvement algorithm (see equation 6.12 and figure 6.1) is used for finding the ideal subset of variables in the GCD criterion. 
Table 6.6: The indicators for the optimal subsets of optimisation variables selected according to the total variance of $k$ PCs and the GCD criterion.

\begin{tabular}{|l|ccc|}
\hline $\boldsymbol{k}$ & $\begin{array}{c}\text { Total Variance of } \\
\boldsymbol{k} \text { PCs } \mathbf{( \% )}\end{array}$ & Indicators & GCD \\
\hline 2 & 83.27 & ESDV type, ESDV spacing & 0.829 \\
3 & 93.26 & ESDV type, ESDV spacing, Number of ESDVs & 0.929 \\
4 & 97.25 & $\begin{array}{c}\text { ESDV type, ESDV combination, ESDV activation } \\
\text { pressure, ESDV spacing }\end{array}$ & 0.963 \\
5 & 9 & $\begin{array}{c}\text { ESDV type, FBR location, Number of ESDVs, } \\
\text { ESDV activation pressure, ESDV combination }\end{array}$ & 0.999 \\
\hline
\end{tabular}

As can be observed in table 6.6, the value of GCD and the total variance of $k$ PCs increase with $k$. According to Cadima et al. (2004), $k$ can be selected with the first $k$ PCs at more than ca. $90 \%$ of variance. It should be noted that with the increase in $k$, the marginal increase in total variance decreases and thus there is no significant increase in total variance observed above a certain threshold. However, the computational complexity increases rapidly as a result of the increase in $k$. Therefore, $k=3$ is selected here corresponding to the total variance of 3 PCs of $93.27 \%$.

Turning to the GCD, when $k=3$, the GCD between the subspaces spanned by the first three PCs and the optimisation variables equals 0.929, showing close agreement with the total variance. By using the Restricted Improvement algorithm, the indicators for the optimal set of optimisation variables are determined as ESDV type, ESDV spacing and number of ESDVs. For the total variance method, to choose 3 optimal variables from a total 6 variables, there are 63 possible subsets to test. The application of the GCD criterion showcases a noticeable computational simplification as compared to the total variance method that could be employed in this case study. 


\subsubsection{Multi-objective Optimisation Results}

Figure 6.5 shows the variation of normalised total inventory loss, $J_{1}$ as a function of normalised total annual valve cost, $J_{2}$. The normalised value is calculated using equations 6.4 and 6.5 . The objective function $J_{3}$, which represents the ESDVs total failure rate, is marked in different colours for the data points. Each data point describes one ESDV configuration. The colours for the data points are on a scale from green (which represents 0 /demand for total failure rate) to red (which represents 0.5 /demand for total failure rate).

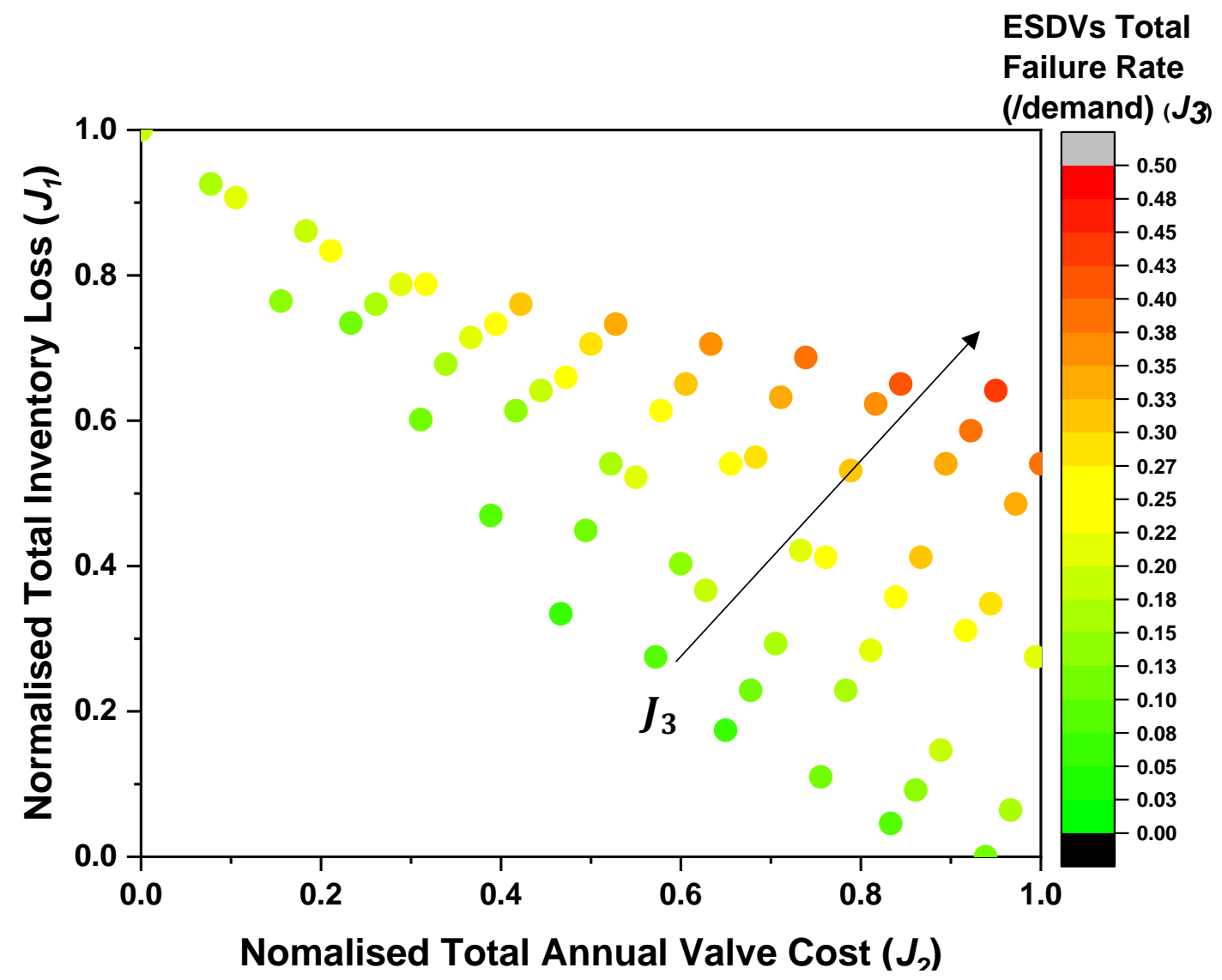

Figure 6.5: ESDV multi-objective optimisation results: Variation of normalised total inventory loss $\left(J_{1}\right)$ with normalised total annual valve cost $\left(J_{2}\right)$. The colour of each data point represents ESDVs total failure rate on demand $\left(J_{3}\right)$ as indicated in the vertical column.

As can be seen from figure $6.5, J_{1}$ shows the expected general decrease as $J_{2}$ increases from 0 to 1 . $J_{1}$ drops from its maximum value 1 at $J_{2}=0$ and approaches ca. $0-0.6$ at $J_{2}=1$. It is noteworthy that as $J_{2}$ increases, the choices for ESDV configurations (i.e. ESDV type, ESDV 
spacing and number of ESDVs) in one cost range increases significantly. For example, the number of possible ESDV configurations increases from 2 to 9 when $J_{2}$ increases from the range $[0,0.1]$ to $[0.9,1]$. The objective function $J_{3}$, on the other hand, shows a general increase as both $J_{1}$ and $J_{2}$ increasing from 0 to 1 .

In order to help decision makers to select the optimal set of ESDV configuration, figure 6.5 can be further analysed based on various perspectives. For example, decision makers can choose the optimal set from a certain range of total ESDV costs or below a certain threshold for the total inventory loss.

The figures 6.6 and 6.7 demonstrate how the proposed optimal set selection examples depend on two selected important perspectives, including normalised total annual valve cost and normalised total inventory loss.

Figure 6.6 presents the ESDV multi-objective optimisation results focusing on normalised total annual valve $\operatorname{cost}\left(J_{2}\right)$ in the chosen range of 0.9 to 1 . As it may be observed, there are 9 different possible ESDV configurations, with $J_{1}$ varying from 0 to ca. 0.64 and $J_{3}$ increasing from ca. 0.11 /demand to 0.45 /demand.

The worst case and best case scenario data sets here are marked by points A and B, respectively. Point A, representing the ESDV configuration for $15 \mathrm{RCV}$ s only with an average valve distance of ca. $9.5 \mathrm{~km}$, has the highest values of $J_{1}$ (ca. 0.64) and $J_{3}$ (ca. $0.45 /$ demand) at a normalised total annual valve cost of ca. 0.95. Point B represents the ESDV configuration for a mix of 8 ASVs and $1 \mathrm{RCV}$ with an average valve distance of ca. $15 \mathrm{~km}$. Point B has the lowest values of $J_{1}(0)$ and $J_{3}$ (ca. 0.12 /demand) at a normalised total annual valve cost of ca. 0.94 . Therefore, it can be concluded that point $\mathrm{B}$ in figure 6.6 is the optimal set for the ESDV configuration when $J_{2}$ is in the chosen range of 0.9 to 1 . 


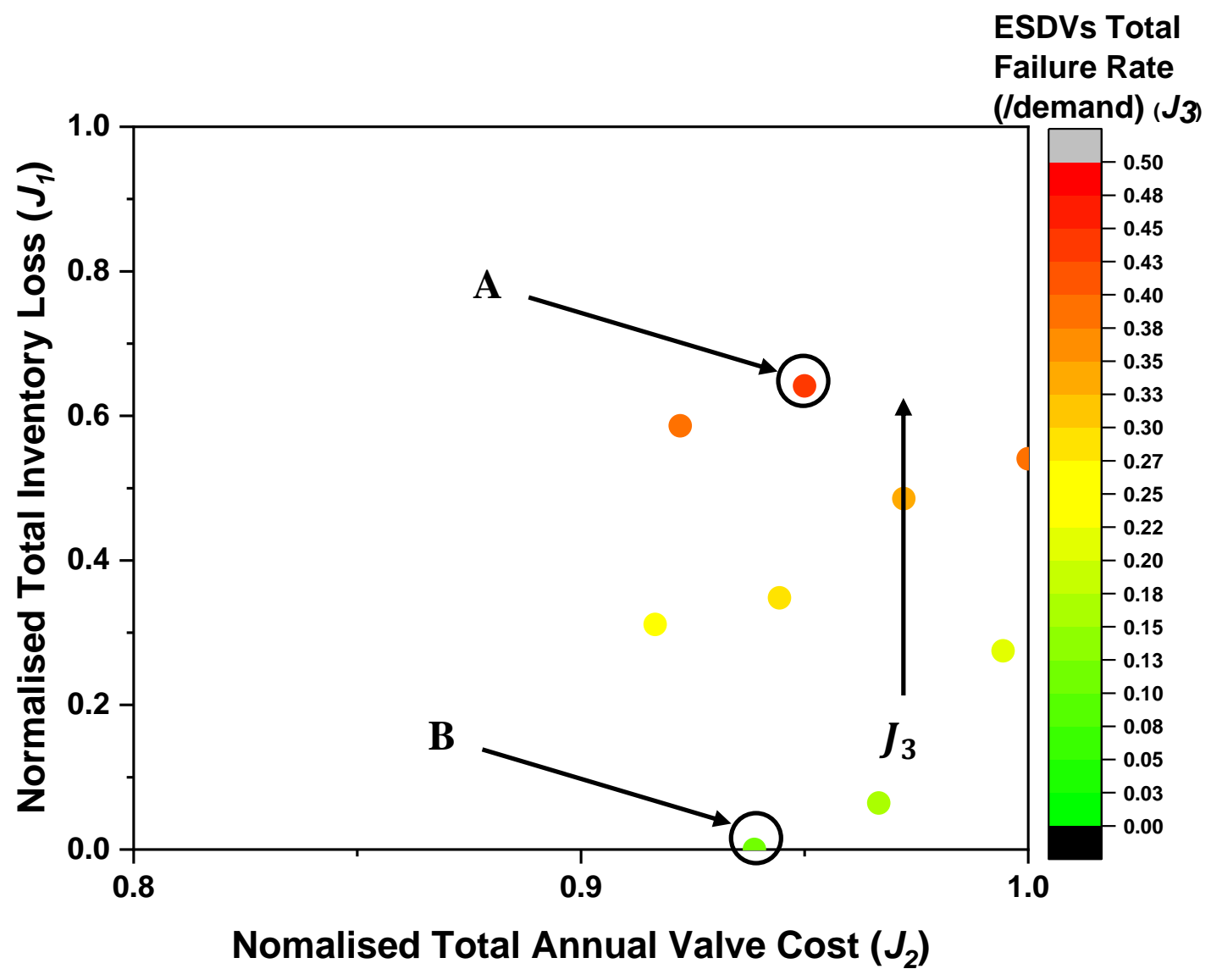

Figure 6.6: ESDV multi-objective optimisation results: Variation of normalised total inventory loss $\left(J_{1}\right)$ with normalised total annual valve cost $\left(J_{2}\right)$ in the range of 0.9 to 1 . The colour of each data point represents ESDVs total failure rate on demand $\left(J_{3}\right)$ as indicated in the vertical column.

In the second optimal set selection example, it is assumed that the decision maker considers the hazard associated with a pipeline failure (i.e. normalised total inventory cost) as the priority aspect. Figure 6.7 demonstrates the ESDV multi-objective optimisation results focusing on normalised total inventory loss $\left(J_{1}\right)$ below the threshold of 0.5 . As it may be observed, there are 25 different possible ESDV configurations in total matching this criterion. 


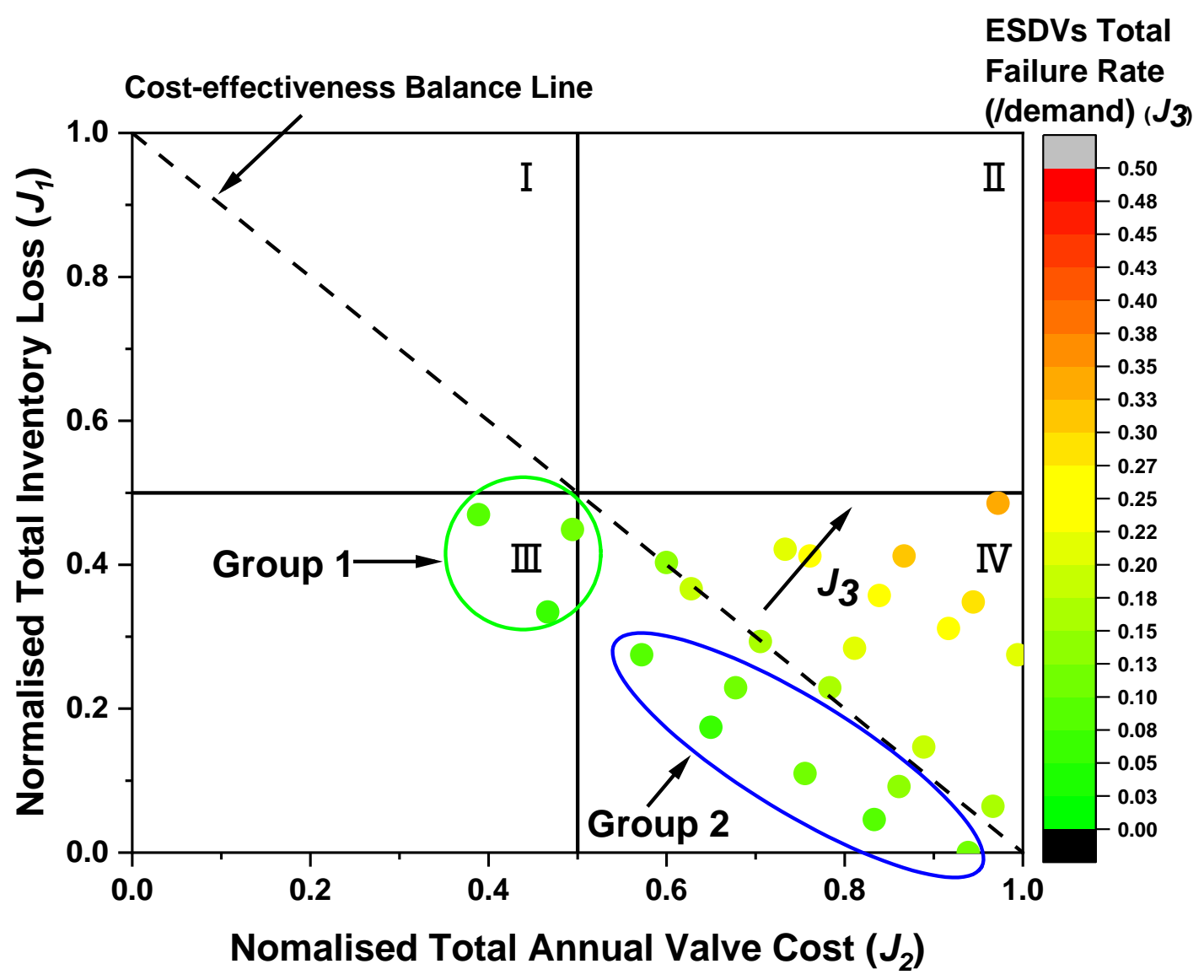

Figure 6.7: ESDV multi-objective optimisation results: Variation of normalised total inventory loss $\left(J_{1}\right)$ (below 0.5 ) with normalised total annual valve cost $\left(J_{2}\right)$. The colour of each data point represents ESDVs total failure rate on demand $\left(J_{3}\right)$ as indicated in the vertical column.

In order to decide the optimal set, cost-effectiveness analysis can be introduced. In figure 6.7, the coordinate system is divided into 4 different zones: I, II, III and IV. Zone I and zone IV respectively represent the scenarios for cost saving; low effectiveness; low/medium failure rate and cost increasing; high effectiveness; low-medium failure rate. The data sets in these two zones may be potentially accepted by the decision makers.

Zone II describes the scenario of valve cost increasing; low effectiveness; medium-high failure rate. As such, the data sets in this zone need to be avoided. In contrast, zone III displays the scenario of the greatest cost saving; high effectiveness and low failure rate with the data sets in this zone. As such, it should be preferably considered as optimal sets. It can be readily seen 
from figure 6.7 that the 25 different possible ESDV configurations lie on two different zones: zone III with 3 possible configurations and zone IV with 22 possible configurations. As such, the 3 data sets in zone III (namely group 1) should be considered as optimal solutions.

For the purpose of evaluating the data sets in zone IV, the cost-effectiveness balance line is also presented in the figure (marked as a dashed line). The data sets above the line should be considered as less cost-effective and should be therefore avoided by the decision makers. On the other hand, the data sets below the line can be recognised as more cost-effective and may be considered as optimal sets. Therefore, there are 7 possible ESDV configurations under the cost-effectiveness balance line in zone IV (namely group 2) which may be favoured by decision makers. It is noteworthy that $J_{2}$ depends highly on the single valve cost, $V_{P N}$ (in this study the value used are $V_{P N}(A S V)=£ 140000$ and $V_{P N}(R C V)=£ 60000 ;$ CPPEC, 2012). Thus more optimal sets (i.e. data sets in Zones III and IV) are expected with a lower $V_{P N}$, and less optimal sets are expected with a higher $V_{P N}$.

\subsection{Concluding Remarks}

This chapter described the development and application of a comprehensive methodology combining multi-objective optimisation and Principal Component Analysis (PCA) for selecting optimal inline ESDV configurations for high-pressure pipelines striking a reasonable balance between risk reduction and valve cost. Such type of capability is fundamentally important given the huge quantities of highly hazardous materials being transported across the globe using high-pressure pipelines.

The fundamental theory of the multi-objective optimisation method and PCA, including the Yanai's Generalized Coefficient of Determination (GCD) criterion and the Restricted Improvement algorithm was first presented. A real planned, $1016 \mathrm{~mm}$ i.d., $150.2 \mathrm{~km}$ long natural gas transmission pipeline in China was chosen as the case study. Given that the pipeline is destined to pass through several populous cities, its safe operation is of paramount importance.

Based on the results and analysis presented for the specific case study, the following key conclusions may be made: 
- Having identified a necessary set of 6 optimisation variables for ESDV configurations, PCA was firstly applied to reduce the number of redundant optimisation variables. It was found that only for the first two PCs, their eigenvalues were greater than 1, which made the data set of variables essential in two-dimensional analysis. The first two PCs accounted $83.27 \%$ of the total variability.

- Both the GCD criterion and the total variance associated with the first $k$ PCs were used to select the most promising subsets of optimisation variables from $k$ indicators. It was found that with the increase in $k$, the marginal increase in total variance decreased and thus there was no significant increase in total variance observed above a certain threshold. In this study, $k=3$ was selected according to Cadima's criteria (2004) and the total variance of 3 PCs was $93.27 \%$.

- Turning to the GCD, when $k=3$, the GCD was equal to 0.929 , showing close agreement with the total variance. By using the Restricted Improvement algorithm, the indicators for the optimal set of optimisation variables were determined as ESDV type, ESDV spacing and number of ESDVs. For the total variance method, 63 possible subsets were tested in order to choose 3 optimal variables from 6 optimisation variables. The application of GCD criterion showed a significant computational simplification as compared to the total variance method.

- Following the results of PCA, the selected 3 optimisation variables were applied into the multi-objective optimisation. The results of multi-objective optimisation were presented using scatter plots providing a geometrical visualisation of the Pareto Front and Set. It was found that normalised total inventory loss decreased as normalised total annual valve cost increased. In contrast, ESDV total failure rate showed a general growth with the increasing of both normalised total inventory loss and total annual valve cost.

- In order to help decision makers to select the optimal set of ESDV configuration, two examples were given to further analyse the multi-objective optimisation results based on two perspectives: from a certain range of total ESDV costs and below a certain threshold of the inventory loss. The first example focused on normalised total annual valve cost in the chosen range of 0.9 to 1 . It was found that the optimal ESDV 
configuration was a mix of $8 \mathrm{ASV}$ and $1 \mathrm{RCV}$ and an average valve distance of ca. 15 $\mathrm{km}$, which had the lowest values of the total inventory loss prior to complete valve closure following FBR failure and the total ESDV failure rate at a normalised total annual valve cost of ca. 0.94 .

- The second example targeted on the multi-objective optimisation results with the normalised total inventory loss below the assumed threshold of 0.5 . Cost-effectiveness analysis was introduced to decide the optimal sets. Two groups of data sets were highlighted as the optimal solutions: the first included 3 ESDV configurations in zone III, representing the scenario with the greatest cost saving; high effectiveness and low failure rate and should be preferably considered by decision makers. In the second group, 7 possible ESDV configurations under the cost-effectiveness balance line in zone IV, representing the scenario for cost saving; less effectiveness and low-medium failure rate may be considered by decision makers as the optimal solutions.

In conclusion, the methodology developed in this chapter provides a fundamental modelling technique for complex multi-objective problems and the results can serve as a highly informative tool in practice. It successfully demonstrates the capability of employing the variable reduction techniques in the multi-objective optimisation study, with emphasis on computational simplification whilst still retaining good agreement with the output of a realworld multi-objective problem. The results of the case study can assist decision makers to select the optimal inline ESDV configuration including the ESDV type, number and spacing as well as their combinations and operational settings for striking a balance among three objectives: the amount of total inventory loss prior to complete valve closure following FBR, the ESDV capital cost and the total failure rate for all ESDVs employed along the pipeline. 


\section{Chapter 7:}

\section{Conclusions and Future Work}

\subsection{Conclusions}

High-pressure pipelines are the primary mode of choice for transporting large quantities of hazardous fluids across the globe. The failure of such pipelines can lead to the release of significant amounts of flammable or toxic inventories, which may in turn present risks to life, environment and property. In order to mitigate such risks, various types of emergency shutdown valves (ESDVs), including Check Valves (CVs), Automatic Shut-off Valves (ASVs) and Remote Control Valves (RCVs), are installed along high-pressure pipelines as the frontline emergency mitigation tool. Given the significant capital cost of ESDVs, which can become prohibitive depending on their type and number of units employed, their routine maintenance which can be especially challenging for offshore operations, the optimum inline ESDV configuration must be carefully determined to remain cost effective whilst providing a reasonably acceptable level of protection in the event of a pipeline failure.

In light of the above, accounting for the critically important ensuing in-pipe transient fluid flow behaviour based on the Homogeneous Equilibrium Mixture (HEM) assumption, this thesis described the development of a rigorous and computationally efficient multi-objective optimisation model for selecting the optimal ESDV configuration for pressurised transportation pipelines. The model accounts for the valve type and combinations, cost, number of installed units and their failure frequencies. Its development, application and testing involved the following steps:

- modelling and study of the dynamic response of ESDVs following the accidental failure of high-pressure pipelines, including:

i. the investigation of pressure surges upon CV closure following Full Bore Rupture (FBR) of high-pressure pipelines

ii. the investigation of $\mathrm{ASV}$ and $\mathrm{RCV}$ activation pressure on the efficacy in isolating the flow following pipeline failure 
- simulating and analysing the efficacy of ESDVs using different inline valve combinations and spacings following the pipeline failure

- developing a multi-objective optimisation method combined with Principal Component Analysis (PCA) and applying it to a real high-pressure transmission pipeline to determine the optimal inline ESDV configuration striking a balance between three objectives:

i. the ESDV capital cost

ii. the ESDVs failure rate (frequency)

iii. the amount of total inventory escaping prior to complete valve closure following pipeline failure

The following is a summary of the main findings in each chapter:

In Chapter 2, the theoretical background for modelling pipeline transient flow, the numerical solution applied to solve the conservation equations and the pertinent boundary conditions were presented and discussed. The included the mass, momentum and energy conservation equations, the various hydrodynamic and thermodynamic relations for predicting the pertinent fluid properties, the Peng-Robinson Equation of State, the steady state isothermal flow model, the formulation of the Method of Characteristics and various boundary conditions to model the fluid as well as ESDV closure dynamics following pipeline failure.

Also included in this chapter was a detailed review of the relevant studies focusing on the applications of the HEM model for predicting high-pressure pipeline decompression behaviour. Based on the review, the HEM model was shown to perform reasonably well as compared to measured data for pipeline decompression tests. Moreover, the HEM model was shown to have been successfully applied for investigating the dynamic response of ESDVs through the implementation of the appropriate boundary conditions.

No studies were found to have addressed the fundamentally important questions of practical significance such as the resulting pressure surges upon CV closure, the impact of ASV and RCV activation pressure in isolating the outflow flow following pipeline failure, and 
importantly, the efficacy of using different combinations of inline ESDV in limiting the amount of the inventory escaping prior to complete valve closure.

Chapter 3 presented a review of multi-objective optimisation methods, including the general approaches to select the solutions, the specific techniques to visualise the Pareto Front and a survey of the applications of multi-objective optimisation methods. The review highlighted considerable progress has been made in applying multi-objective optimisation methods in providing a set of optimal solution for pipeline network design and operation. Very limited previous work involving the application of such methods to inline ESDV pipeline configurations was found.

In the same vein, Brown et al. (2014) presented a multi-objective optimisation study balancing valve spacing in $\mathrm{CO}_{2}$ pipelines as a trade-off between risk reduction versus ESDV installation and maintenance costs. However, with the exception of the valve spacing, other important parameters related with ESDV configuration such as ESDV type \& their combination, number and operational settings (e.g. activation \& closure times) were not considered.

Chapter 4 focused on modelling followed by comparing and contrasting the dynamic response of different types of ESDVs following the accidental failure of high-pressure pipelines. An ethylene pipeline was chosen as a case study given the chemical's extensive pipeline transportation across the globe and its hazardous nature as being flammable, explosive and potentially toxic at high concentrations.

In the first part of this chapter, the investigation of the resulting transient pressure surges upon $\mathrm{CV}$ closure for a wide range of ethylene pipeline operating pressures and temperatures was carried out. It was found that the pressure surge magnitude generally decreased with the operating pressure and temperature, varying non-linearly with the valve closure delay time. Remarkably, it was found that for the ethylene pipeline test case operating at a pressure of 90 bar, the maximum amplitude of the pressure surge reached ca. 32 bar. This could potently lead to a sufficiently large thrust or bending forces acting on the pipeline segments risking undermining the pipeline mechanical integrity.

In the second part of Chapter 4, the impact of the magnitude of ASV and RCV activation pressure on the total amount of inventory loss following pipeline failure occurring in between two ESDVs along the ethylene pipeline was investigated. The simulation tests involved FBR 
and different generic sizes of puncture failures at three failure locations. It was found that for different failure locations, the ASV activation time was mainly affected by the distance between the valve and the rupture plane, and not the initial in-pipe flow.

In the case of FBR and large puncture diameters failures (i.e. $\geq 50 \%$ of pipe i.d.), decreasing valve activation pressure resulted in a reduction in the total inventory loss prior to complete valve closure. However, the reduction in the total inventory loss did not change significantly with a decrease in the valve activation pressure. Turning to a small puncture (i.e. $10 \mathrm{~mm}$ ), for the cases studied, both ASVs and RCVs failed to activate for relatively large valve activation pressures (i.e. more than 2.5 bar).

Investigations involving comparisons based on using either ASVs or RCVs along the pipeline showed that ASVs offered significantly higher reduction in the total inventory loss prior to complete isolation as compared to RCVs. This was due to the additional operator action time required for the activation of RCVs, during which large amount of fluid would escape from the pipeline. However, using ASVs became less favourable as puncture size decreased. In such circumstances, the capital and operating costs of either of the valves will also be important factors governing their selection.

In Chapter 5, the efficacies in minimising the total amount of inventory escaping based on the use of different ESDV combinations and spacing following pipeline FBR and puncture were investigated. Two typical high-pressure onshore pipelines were selected as case studies; one transporting ethylene (highly flammable, explosive and potentially toxic at high concentrations), the other transporting $\mathrm{CO}_{2}$ (an asphyxiant at concentrations $>=7 \% \mathrm{v} / \mathrm{v}$ ).

Two sets of investigations were performed. The first included the impact of RCV and CV spacing in minimising outflow following a puncture failure mid-way along a hypothetical $\mathrm{CO}_{2}$ pipeline. It was found that the installation of two equidistant $\mathrm{CVs}$ along the pipeline progressively resulted in a reduction of the amount of cumulative mass discharged by ca. $50 \%$. However, further increase in the number of CVs beyond 2 had little effect on further reducing the amount of cumulative mass discharged. Switching to RCV placed at both upstream and downstream of the puncture, no such limit was observed. However, the reduction obtained by increasing the number of RCVs became marginal above certain range due to the long valve activation time resulting in significant amount of inventory loss prior to valve activation. 
In the case of using both RCVs and CVs at the same location (i.e. in tandem), significant improvement was observed in minimising outflow compared to only using one type of valve. This demonstrated the potential merits in the choice of using different type of valves at the same locations for ESDV optimisation.

Next, the efficacies of ASVs, RCVs and CVs and their combinations were assessed by comparing the amount of total inventory loss following FBR located between two ESDVs along a hypothetical ethylene pipeline. It was found that for two ASVs installed along the pipeline, the amount of total inventory loss matched closely (within ca. +-10\%) with initial mass of inventory prior to failure. This indicated that using ASVs enabled relatively fast isolation of the pipeline segment following FBR.

Compared to using ASVs only, the combination of ASV and CV showed no significant impact on the reduction in the amount of total inventory loss and hence the duration of release following FBR. As such, the cost and reliability of emergency isolation systems should be carefully considered when adding CVs to pipelines already equipped with ASVs.

In the case of combining $\mathrm{RCV}$ and $\mathrm{CV}$, significant reductions in the total inventory loss as compared to emergency isolation based on RCVs only was observed. As such, installing additional $\mathrm{CV}$ s in high-pressure pipelines equipped with RCVs, could significantly reduce the duration of release, hence assisting faster accident consequence mitigation.

Chapter 6 described the development and application of a comprehensive methodology combining multi-objective optimisation and PCA for selecting optimal inline ESDV configurations for high-pressure pipelines striking a balance among three objectives, namely the amount of total inventory escaping prior to complete valve closure following pipeline failure, the ESDV capital cost and the ESDVs failure rate. The background theory of the multiobjective optimisation method and PCA, including the Yanai's Generalized Coefficient of Determination (GCD) criterion and the Restricted Improvement algorithm was first presented. This was followed by the application of the constructed model to a real planned, $1016 \mathrm{~mm}$ i.d., $150.2 \mathrm{~km}$ long natural gas transmission pipeline in China. Given that the pipeline is destined to pass through several populous cities, its safe operation is of paramount importance.

Having identified a necessary set of 6 optimisation variables for ESDV configurations, PCA was firstly applied to reduce the number of redundant optimisation variables. It was found that 
only for the first two Principal Components (PCs), their eigenvalues were greater than 1, thus making the data set of the essential optimisation variables in two-dimensional analysis. The first two PCs accounted $83.27 \%$ of the total variability.

Both the GCD criterion and the total variance method were used to select the most promising subsets of optimisation variables from $k$ indicators. It was found that with the increase in $k$, the marginal increase in total variance decreased and thus there was no significant increase in total variance observed above a certain threshold. In this study, $k=3$ was selected according to Cadima's criteria (2004) and the total variance of 3 PCs was $93.27 \%$.

Turning to the GCD, when $k=3$, the GCD was equal to 0.929 , showing close agreement with the total variance. By using the Restricted Improvement algorithm, the optimal set of optimisation variables were determined as ESDV type, ESDV spacing and number of ESDVs. For the total variance method, 63 possible subsets were tested in order to choose 3 optimal variables. The application of GCD criterion showed a significant computational simplification as compared to the total variance method.

Following the results of PCA, the selected 3 optimisation variables were applied into the multiobjective optimisation. The results of multi-objective optimisation were presented using scatter plots providing a geometrical visualisation of the Pareto Front and Set. It was found that the normalised total inventory loss decreased as normalised total annual valve cost increased. In contrast, the ESDV total failure rate showed a general growth with the increasing of both normalised total inventory loss and total annual valve cost.

In order to select the optimal set of ESDV configuration, two optimal set selection examples were given to further analyse the multi-objective optimisation results based on two selected important perspectives: from a certain range of total ESDV costs and below a certain threshold of the inventory loss. The first example focused on the normalised total annual valve cost in the chosen range of 0.9 to 1 . It was found that the optimal ESDV configuration was a mix of 8 ASVs and $1 \mathrm{RCV}$ with an average valve distance of ca. $15 \mathrm{~km}$, which had the lowest values of the total ESDV failure rate and the total inventory loss prior to complete valve closure following FBR failure at a normalised total annual valve cost of ca. 0.94.

The second example targeted on the multi-objective optimisation results with the normalised total inventory loss below the assumed threshold of 0.5 . In order to decide the optimal sets, 
cost-effectiveness analysis was introduced, and the coordinate system was divided into 4 different zones: I, II, III and IV. Two groups of data sets were highlighted as the optimal solutions: the first included 3 ESDV configurations in zone III, representing the scenario with the greatest cost saving; the most effective and low failure rate, should be preferably considered by decision makers. In the second group, 7 possible ESDV configurations under the costeffectiveness balance line in zone IV, representing the scenario for cost saving; less effective and low-medium failure rate, which may be considered as the optimal solutions.

In conclusion, the mathematical tools, the systematic investigations and findings reported in this work are of paramount importance in practice, assisting pipeline operators to select the optimal ESDV configuration balancing safety against cost taking account of a myriad of design and operating parameters. A knowledge of the subsequent predicted time variant amount of inventory escaping following emergency isolation for the optimised ESDV configuration is particularly important as it serves as the source term for determining the consequences of the pipeline failure. Depending on the type of fluid being transported, these include radiative heat flux as result of a fire, explosion over-pressure, toxic dosage and environmental pollution. Such data ultimately dictate the minimum pipeline safe distances to populated areas and emergency response planning.

In conclusion, the key outcome of this $\mathrm{PhD}$ study is the development of a robust analytical decision making tool for improving the safety of high-pressure transportation pipelines, thus protecting life, property and the environment. 


\subsection{Suggestions for Future Work}

\subsubsection{Leak Detection Modelling}

In Chapter 4, it was found that for a small puncture (i.e. $10 \mathrm{~mm}$ ), ASVs and RCVs might not activate due to the fact that the pressure drop at the valve location was relatively small (i.e. less than 2.5 bar) throughout the decompression. In practice, a pipeline leak, especially a slow leak following a small size puncture from a buried pipeline, can be very difficult to detect (Henrie et al., 2016). Such pipeline failure can lead to the continuous release of significant amounts of flammable or toxic inventories before it is detected, which may in turn present significant risks to life, environment and property. As such, future work should involve the development of a leak detection model for high-pressure transportation pipelines focusing on small puncture failures. The fast and accurate detection of leaks from such failures allows for efficient containment strategies such as the quick response for the ESDV closure, hence minimising the resulting cost and damage.

\subsubsection{Reliability Analysis for Other Control Devices}

The multi-objective optimisation study presented in this study primarily focused on ESDV itself (e.g. its type, number, closure dynamic, failure rate, capital cost, etc.). In practice, the reliabilities of other control devices installed along the pipeline may also influence the determination of the optimal ESDV configuration. For example, in high-pressure pipelines, compressor and booster stations are usually equipped with ESDVs and other control devices including joints, sensors and flow meters can influence the pipeline integrity (Grigoriev and Grigorieva, 2009). Therefore, the further extension of the current ESDV multi-objective optimisation method accounting for the reliability analysis of these control devices as an objective function would be useful. 


\subsubsection{Combining Pipeline Maintenance Optimisation Models with ESDV Multi-Objective Optimisation Study}

In order to maintain safe operation of high-pressure pipelines, appropriate maintenance optimisation strategies are required to maximise reliability. A number of such studies can be found in open literature (see for example Castanier and Rausand, 2006; Gong and Zhou, 2018; Chin et al., 2020). As part of future work, a combined approach involving pipeline maintenance optimisation strategies and the current ESDV multi-objective optimisation model would be extremely useful. 


\section{References}

Abraham, A. and Jain, L. (2005) 'Evolutionary Multiobjective Optimization', in Evolutionary Multiobjective Optimization. London: Springer-Verlag, pp. 1-6. doi: 10.1007/1-84628-1377_1.

Akbari, M., Asadi, P., Besharati Givi, M. K. and Khodabandehlouie, G. (2014) 'Artificial neural network and optimization', in Givi, M. K. B. and Asadi, Parviz (eds) Advances in Friction-Stir Welding and Processing. Woodhead Publishing, pp. 543-599. doi: 10.1533/9780857094551.543.

Alves, F. da S., Souza, J. N. M. de and Costa, A. L. H. (2016) 'Multi-objective design optimization of natural gas transmission networks', Computers and Chemical Engineering, 93, pp. 212-220. doi: 10.1016/j.compchemeng.2016.06.006.

American Lifelines Alliance (2001) Guidelines for the design of buried steel pipe, American Socoiety of Civil Engineers. Available at: https://www.americanlifelinesalliance.com/pdf/Update061305.pdf.

Angielczyk, W., Seynhaeve, J., Butrymowicz, D. and Bartosiewicz, Y. (2010) '1-D modeling of supersonic carbon dioxide two-phase flow through ejector motive nozzle', International Refrigeration and Air Conditioning Conference at Purdue, pp. 1-8.

Arya, V., Garg, N., Khandekar, R., Meyerson, A., Munagala, K. and Pandit, V. (2004) 'Local search heuristics for k -median and facility location problems', SIAM Journal on Computing, 33(3), pp. 544-562. doi: 10.1137/S0097539702416402.

Atti, O. F. (2006) Modelling outflow and low temperature induced crack propagation in pressurised pipelines. $\mathrm{PhD}$ thesis. University College London.

Bechikh, S., Kessentini, M., Said, L. Ben and Ghédira, K. (2015) 'Preference incorporation in Evolutionary Multiobjective Optimization: A Survey of the State-of-the-Art', in Hurson, A. R. (ed.) Advances in Computers. Elsevier, pp. 141-207. doi: 10.1016/bs.adcom.2015.03.001.

Bendlksen, K. H., Maines, D., Moe, R. and Nuland, S. (2004) 'The dynamic two-fluid model OLGA: Theory and application', SPE Reprint Series, (58), pp. 52-61. 
Bilicki, Z. and Joseph, K. (1990) 'Physical aspects of the relaxation model in two-phase flow', Proceedings of the Royal Society of London. A. Mathematical and Physical Sciences, 428(1875), pp. 379-397. doi: 10.1098/rspa.1990.0040.

Blasco, X., Herrero, J. M., Sanchis, J. and Martínez, M. (2008) 'A new graphical visualization of n-dimensional Pareto front for decision-making in multiobjective optimization', Information Sciences, 178(20), pp. 3908-3924. doi: 10.1016/j.ins.2008.06.010.

Botros, K. K., Geerligs, J., Zhou, J. and Glover, A. (2007) 'Measurements of flow parameters and decompression wave speed following rupture of rich gas pipelines, and comparison with GASDECOM', International Journal of Pressure Vessels and Piping, 84(6), pp. 358-367. doi: 10.1016/j.ijpvp.2007.01.005.

Brent, R. P. (1971) 'An algorithm with guaranteed convergence for finding a zero of a function', in The Computer Journal, pp. 422-425. doi: 10.1093/comjn1/14.4.422.

Brown, S. (2011) CFD modelling of outflow and ductile fracture propagation in pressurised pipelines. PhD thesis. University College London.

Brown, S., Martynov, S., Mahgerefteh, H. and Proust, C. (2013) 'A homogeneous relaxation flow model for the full bore rupture of dense phase $\mathrm{CO}_{2}$ pipelines', International Journal of Greenhouse Gas Control, 17, pp. 349-356. doi: 10.1016/j.ijggc.2013.05.020.

Brown, S., Martynov, S., Mahgerefteh, H., Chen, S. and Zhang, Y. (2014) 'Modelling the nonequilibrium two-phase flow during depressurisation of $\mathrm{CO}_{2}$ pipelines', International Journal of Greenhouse Gas Control, 30, pp. 9-18. doi: 10.1016/j.ijggc.2014.08.013.

Brown, Solomon, Sundara, V., Martynov, S. and Mahgerefteh, H. (2014) 'Optimal valve spacing for next generation $\mathrm{CO}_{2}$ pipelines', in Computer Aided Chemical Engineering, pp. 265-270. doi: 10.1016/B978-0-444-63456-6.50045-4.

Cadima, J., Cerdeira, J. O. and Minhoto, M. (2004) 'Computational aspects of algorithms for variable selection in the context of principal components', Computational Statistics and Data Analysis, 47(2 SPEC. ISS.), pp. 225-236. doi: 10.1016/j.csda.2003.11.001.

Cadima, J. F. C. L. and Jolliffe, I. T. (2001) 'Variable selection and the interpretation of principal subspaces', Journal of Agricultural, Biological, and Environmental Statistics, 6(1), 
pp. 62-78. doi: 10.1198/108571101300325256.

Carbon Capture \& Storage Association (2020) The sixth carbon budget \& Welsh emissions targets Call for Evidence. Available at: https://www.ccsassociation.org/resources/ (Accessed: 15 March 2021).

Castanier, B. and Rausand, M. (2006) 'Maintenance optimization for subsea oil pipelines', International Journal of Pressure Vessels and Piping, 83(4), pp. 236-243. doi: 10.1016/j.ijpvp.2006.02.013.

Chen, J. (1993) Modelling of transient flow in pipeline blowdown problems, Chemical Technology. Available at: http://opensigle.inist.fr/handle/10068/695603.

Chen, J., Richardson, S. and Saville, G. (1992) 'Numerical simulation of full-bore ruptures of pipelines containing perfect gases', Process Safety and Environmental Protection, 70(b), pp. 59-69. Available at: http://archive.icheme.org/cgibin/somsid.cgi?type=header\&amp;record=2388.

Chen, N. H. (1979) 'An explicit equation for friction factor in pipe', Industrial and Engineering Chemistry Fundamentals, 18(3), pp. 296-297. doi: 10.1021/i160071a019.

Chern, M. J., Wang, C. C. and Ma, C. H. (2007) 'Performance test and flow visualization of ball valve', Experimental Thermal and Fluid Science, 31(6), pp. 505-512. doi: 10.1016/j.expthermflusci.2006.04.019.

Chin, H. H., Varbanov, P. S., Klemeš, J. J., Benjamin, M. F. D. and Tan, R. R. (2020) 'Asset maintenance optimisation approaches in the chemical and process industries - A review', Chemical Engineering Research and Design, 164, pp. 162-194. doi: 10.1016/j.cherd.2020.09.034.

China Statistics Press (2019) China statistical yearbook 2019, National Bureau of Statistics of the People's Republic of China.

Cleaver, R. P., Cumber, P. S. and Halford, A. (2003) 'Modelling outflow from a ruptured pipeline transporting compressed volatile liquids', Journal of Loss Prevention in the Process Industries, 16(6), pp. 533-543. doi: 10.1016/j.jlp.2003.08.004. 
Corne, D. W. and Knowles, J. D. (2007) 'Techniques for highly multiobjective optimisation', in Proceedings of the 9th annual conference on Genetic and evolutionary computation GECCO '07. New York, New York, USA: ACM Press, p. 773. doi: $10.1145 / 1276958.1277115$.

Courant, R., Friedrichs, K. and Lewy, H. (2002) 'On the Partial Difference Equations of mathematical physics', Hans Lewy Selecta, pp. 59-78. doi: 10.1007/978-1-4612-2080-0_6.

CPPEC (2012) Feasibility study report - Harbin-Shenyang pipeline, Unpublished Technical Report.

Crawley, F. K., Lines, I. G. and Mather, J. (2003) 'Oil and gas pipeline failure modelling', Process Safety and Environmental Protection, 81(1), pp. 3-11. doi: $10.1205 / 095758203762851930$.

Cullen, L. (1993) 'The public inquiry into the Piper Alpha disaster', Drilling Contractor; (United States), 49:4.

Deb, K. and Chaudhuri, S. (2005) 'I-EMO: An interactive Evolutionary Multi-objective Optimization tool', in S.K., P., S., B., and S., Biswas (eds) Pattern Recognition and Machine Intelligence. PReMI 2005. Lecture Notes in Computer Science. Berlin, Heidelberg: Springer, pp. 690-695. doi: 10.1007/11590316_111.

Demirbas, A. (2002) 'Fuel properties of hydrogen, liquefied petroleum gas (LPG), and Compressed Natural Gas (CNG) for Transportation', Energy Sources, 24(7), pp. 601-610. doi: 10.1080/00908312.2002.11877434.

Demissie, A. G. (2015) Multi-Objective optimization for natural gas pipeline network operation. $\mathrm{PhD}$ thesis. Lamar University.

Deopura, B. L., Alagirusamy, R., Joshi, M. and Gupta, B. (eds) (2008) Polyesters and Polyamides. 1st edn, Polyesters and Polyamides. 1st edn. Elsevier. doi: $10.1533 / 9781845694609$.

Dong, K., Dong, X. and Ren, X. (2020) 'Can expanding natural gas infrastructure mitigate $\mathrm{CO}_{2}$ emissions? Analysis of heterogeneous and mediation effects for China', Energy Economics, 90. doi: 10.1016/j.eneco.2020.104830. 
Downar-Zapolski, P., Bilicki, Z., Bolle, L. and Franco, J. (1996) 'The non-equilibrium relaxation model for one-dimensional flashing liquid flow', International Journal of Multiphase Flow, 22(3), pp. 473-483. doi: 10.1016/0301-9322(95)00078-X.

Du, D.-Z., Pardalos, P. M. and Wu, W. (2008) 'History of optimization', in Encyclopedia of Optimization. Boston, MA: Springer US, pp. 1538-1542. doi: 10.1007/978-0-387-747590_268.

EPS (2013) EPS ethylene link starts up, Pipeline Coating. Available at: www.eps-pipeline.de.

Fairuzov, Y. V. (1998) 'Blowdown of pipelines carrying flashing liquids', AIChE Journal, 44(2), pp. 245-254. doi: 10.1002/aic.690440203.

Faraci, V. (2006) 'Calculating failure rates of series / parallel networks', Journal of the System Reliability Center.

Ferziger, J. H., Perić, M. and Street, R. L. (2020) Computational methods for fluid dynamics, Computers \& Mathematics with Applications. Cham: Springer International Publishing. doi: 10.1007/978-3-319-99693-6.

Flatt, R. (1986) 'Unsteady compressible flow in long pipelines following a rupture', International Journal for Numerical Methods in Fluids, 6(2), pp. 83-100. doi: 10.1002/fld.1650060204.

Foothills Pipe Lines (Yukon) Ltd (1981) Final report on the test program at the Northern Alberta Burst test facility.

Gao, H., Nie, H. and Li, K. (2019) 'Visualisation of Pareto Front approximation: A short survey and empirical comparisons', in 2019 IEEE Congress on Evolutionary Computation (CEC). IEEE, pp. 1750-1757. doi: 10.1109/CEC.2019.8790298.

Global CCS Institude (2018) Fact sheet - Transporting $\mathrm{CO}_{2}$. Available at: https://www.globalccsinstitute.com/wp-content/uploads/2018/12/Global-CCS-Institute-FactSheet_Transporting-CO2-1.pdf (Accessed: 21 March 2021).

Gong, C. and Zhou, W. (2018) 'Multi-objective maintenance strategy for in-service corroding pipelines using genetic algorithms', Structure and Infrastructure Engineering, 14(11), pp. 
1561-1571. doi: 10.1080/15732479.2018.1459744.

Goodfellow, G. D., Lyons, C. J. and Haswell, J. V (2019) UKOPA pipeline product loss incidents and faults report (1962 - 2017), the United Kingdom Onshore Pipeline Operators' Association. Available at: https://www.ukopa.co.uk/pdfs/UKOPA-11-0076.pdf.

Grigoriev, A. and Grigorieva, N. V. (2009) 'The valve location problem: Minimizing environmental damage of a spill in long oil pipelines', Computers and Industrial Engineering, 57(3), pp. 976-982. doi: 10.1016/j.cie.2009.04.001.

Gungor, K. E. and Winterton, R. H. S. (1986) 'A general correlation for flow boiling in tubes and annuli', International Journal of Heat and Mass Transfer, 29(3), pp. 351-358. doi: 10.1016/0017-9310(86)90205-X.

Gutiérrez, E., Lozano, S., Moreira, M. T. and Feijoo, G. (2010) 'Assessing relationships among life-cycle environmental impacts with dimension reduction techniques', Journal of Environmental Management. doi: 10.1016/j.jenvman.2009.12.009.

Harper, P. (2011) Assessment of the major hazard potential of carbon dioxide (CO $\left.\mathrm{CO}_{2}\right)$, Health and Safety Executive. Available at: https://www.hse.gov.uk/carboncapture/assets/docs/majorhazard-potential-carbon-dioxide.pdf (Accessed: 3 February 2021).

Henrie, M., Carpenter, P. and Nicholas, R. E. (2016) Pipeline leak detection handbook, Pipeline Leak Detection Handbook. doi: 10.1016/c2014-0-01914-4.

Hirsch, C. (2007) Numerical computation of internal and external flows, Numerical Computation of Internal and External Flows. Wiley. doi: 10.1016/b978-0-7506-6594-0.x50371.

Hirt, C. W., Amsden, A. A. and Cook, J. L. (1997) 'An Arbitrary Lagrangian-Eulerian computing method for all flow speeds', Journal of Computational Physics, 135(2), pp. 203216. doi: 10.1006/jcph.1997.5702.

HSE (2017) Failure rate and event data for use within risk assessments (06/11/17), Health and Safety Executive. Available at: http://www.hse.gov.uk/landuseplanning/failure-rates.pdf (Accessed: 15 March 2021). 
IMPEL (2009) Lessons learnt from industrial accidents. Seminar in Paris - France, 3-4 June 2009. Available at: www.aria.developpement-durable.gouv.fr (Accessed: 15 December 2020).

Inselberg, A. (2009) Parallel coordinates - Visual multidimensional geometry and its applications. New York, NY: Springer New York. doi: 10.1007/978-0-387-68628-8.

International Energy Agency (2009) Energy Technology Perspectives 2010: Scenarios and Strategies to 2050. Paris: OECD (Energy Technology Perspectives). doi: 10.1787/energy_tech2010-en.

International Energy Agency (2020) Global Energy Review 2020, Global Energy Review 2020. Available at: https://www.iea.org/reports/global-energy-review-2020/global-energy-and-co2emissions-in-2020\#co2-emissions (Accessed: 21 March 2021).

Ishii, M., Hibiki, T., Ishii, M. and Hibiki, T. (2006) 'Basic Relations in Time Averaging', Thermo-Fluid Dynamics of Two-Phase Flow, pp. 67-92. doi: 10.1007/978-0-387-29187-1_4.

Ishii, M. and Hibiki, T. (2011) 'Time Averaged Balance Equation', in Thermo-Fluid Dynamics of Two-Phase Flow. New York, NY: Springer New York, pp. 93-117. doi: 10.1007/978-14419-7985-8_5.

Jolliffe, I. T. (1972) 'Discarding variables in a principal component analysis. I: artificial data', Applied Statistics, 21(2), p. 160. doi: 10.2307/2346488.

Katagiri, H., Sakawa, M., Kato, K. and Nishizaki, I. (2008) 'Interactive multiobjective fuzzy random linear programming: Maximization of possibility and probability', European Journal of Operational Research, 188(2), pp. 530-539. doi: 10.1016/j.ejor.2007.02.050.

Keenan, J. H. and Neumann, E. P. (1946) 'Measurements of Friction in a Pipe for Subsonic and Supersonic Flow of Air', Journal of Applied Mechanics, 13(2), pp. A91-A100. doi: 10.1115/1.4009532.

Keßler, T., Logist, F. and Mangold, M. (2016) 'Use of predictor corrector methods for multiobjective optimization of dynamic systems', in Kravanja, Z. and Bogataj, M. (eds) Computer Aided Chemical Engineering. Elsevier, pp. 313-318. doi: 10.1016/B978-0-444-634283.50057-6. 
Khare, V., Yao, X. and Deb, K. (2003) 'Performance Scaling of Multi-objective Evolutionary Algorithms', in C.M., F., P.J., F., E., Z., L., T., and K., D. (eds) Evolutionary Multi-Criterion Optimization. EMO 2003. Lecture Notes in Computer Science. Berlin, Heidelberg: Springer, pp. 376-390. doi: 10.1007/3-540-36970-8_27.

Kirkpatrick, S., Gelatt, C. D. and Vecchi, M. P. (1983) 'Optimization by Simulated Annealing', Science, 220(4598), pp. 671-680. doi: 10.1126/science.220.4598.671.

Koetzier, H., Kruisbrink, A. C. H. and Lavooij, C. S. W. (1986) 'Dynamic behaviour of large non-return valves', in the 5th international conference on pressure surges, pp. 237-243.

Kohonen, T. (1990) 'The self-organizing map', Proceedings of the IEEE, 78(9), pp. 14641480. doi: 10.1109/5.58325.

Krzanowski, W. (2000) Principles of multivariate analysis: a user's perspective. Oxford University Press.

LeVeque, R. J. (2002) Finite Volume Methods for Hyperbolic Problems, Finite Volume Methods for Hyperbolic Problems. doi: 10.1017/cbo9780511791253.

Lund, H., Flåtten, T. and Tollak Munkejord, S. (2011) 'Depressurization of carbon dioxide in pipelines-Models and methods', Energy Procedia, 4, pp. 2984-2991. doi: 10.1016/j.egypro.2011.02.208.

Mahgerefteh, H., Sundara, V., Brown, S. and Martynov, S. (2016) 'Modelling emergency isolation of carbon dioxide pipelines', International Journal of Greenhouse Gas Control, 44, pp. 88-93. doi: 10.1016/j.ijggc.2015.11.001.

Mahgerefteh, H. and Atti, O. (2006) 'Modeling low-temperature-induced failure of pressurized pipelines', AIChE Journal, 52(3), pp. 1248-1256. doi: 10.1002/aic.10719.

Mahgerefteh, H., Atti, O. and Denton, G. (2007) 'An interpolation technique for rapid CFD simulation of turbulent two-phase flows', Process Safety and Environmental Protection, 85(1 B), pp. 45-50. doi: 10.1205/psep.05118.

Mahgerefteh, H., Brown, S. and Martynov, S. (2012) 'A study of the effects of friction, heat transfer, and stream impurities on the decompression behavior in CO 2 pipelines', Greenhouse 
Gases: Science and Technology, 2(5), pp. 369-379. doi: 10.1002/ghg.1302.

Mahgerefteh, H., Brown, S. and Zhang, P. (2010) 'A dynamic boundary ductilefracturepropagation model for $\mathrm{CO}_{2}$ pipelines', The Journal of Pipeline Engineering, 9(4), pp. $265-276$.

Mahgerefteh, H., Denton, G. and Rykov, Y. (2008) 'A hybrid multiphase flow model', AIChE Journal, 54(9), pp. 2261-2268. doi: 10.1002/aic.11569.

Mahgerefteh, H., Jalali, N. and Fernandez, M. I. (2011) 'When does a vessel become a pipe?', AIChE Journal, 57(12), pp. 3305-3314. doi: 10.1002/aic.12541.

Mahgerefteh, H., Oke, A. and Atti, O. (2006) 'Modelling outflow following rupture in pipeline networks', Chemical Engineering Science, 61(6), pp. 1811-1818. doi: 10.1016/j.ces.2005.10.013.

Mahgerefteh, H., Saha, P. and Economou, I. G. (1997) 'A study of the dynamic response of emergency shutdown valves following full bore rupture of gas pipelines', Process Safety and Environmental Protection, 75(4), pp. 201-209. doi: 10.1205/095758297529039.

Mahgerefteh, H., Saha, P. and Economou, I. G. (1999) 'Fast numerical simulation for full bore rupture of pressurized pipelines', AIChE Journal, 45(6), pp. 1191-1201. doi: 10.1002/aic.690450605.

Mahgerefteh, H., Saha, P. and Economou, I. G. (2000) 'Modeling fluid phase transition effects on dynamic behavior of ESDV', AIChE Journal, 46(5), pp. 997-1006. doi: 10.1002/aic.690460512.

Malakooti, B. (1988) 'A decision support system and a heuristic interactive approach for solving discrete multiple criteria problems', IEEE Transactions on Systems, Man, and Cybernetics, 18(2), pp. 273-284. doi: 10.1109/21.3466.

Marler, R. T. and Arora, J. S. (2004) 'Survey of multi-objective optimization methods for engineering', Structural and Multidisciplinary Optimization, 26(6), pp. 369-395. doi: 10.1007/s00158-003-0368-6.

Martin, O., Otto, S. W. and Felten, E. W. (1992) 'Large-step markov chains for the TSP 
incorporating local search heuristics', Operations Research Letters, 11(4), pp. 219-224. doi: 10.1016/0167-6377(92)90028-2.

Martynov, S., Brown, S., Mahgerefteh, H., Sundara, V., Chen, S. and Zhang, Y. (2014) 'Modelling three-phase releases of carbon dioxide from high-pressure pipelines', Process Safety and Environmental Protection, 92(1), pp. 36-46. doi: 10.1016/j.psep.2013.10.004.

Massey, B. S. and Ward-Smith, J. (1998) Mechanics of fluids. 7th edn. Crc Press.

Medina, H., Arnaldos, J., Casal, J., Bonvicini, S. and Cozzani, V. (2012) 'Risk-based optimization of the design of on-shore pipeline shutdown systems'. doi: 10.1016/j.jlp.2011.12.005.

Mehra, R. K., Heidemann, R. A. and Aziz, K. (1983) 'An accelerated successive substitution algorithm', The Canadian Journal of Chemical Engineering, 61(4), pp. 590-596. doi: $10.1002 /$ cjce.5450610414.

Menikoff, R. (2007) 'Empirical Equations of State for Solids', ShockWave Science and Technology Reference Library, pp. 143-188. doi: 10.1007/978-3-540-68408-4_4.

Menikoff, R. and Plohr, B. J. (1989) 'The Riemann problem for fluid flow of real materials', Reviews of Modern Physics, 61(1), pp. 75-130. doi: 10.1103/RevModPhys.61.75.

Molina, J., Santana, L. V., Hernández-Díaz, A. G., Coello Coello, C. A. and Caballero, R. (2009) 'g-dominance: Reference point based dominance for multiobjective metaheuristics', European Journal of Operational Research, 197(2), pp. 685-692. doi: 10.1016/j.ejor.2008.07.015.

Muhlbauer, W. K. (2004) Pipeline Risk Management Manual: Ideas, Techniques, and Resources. $\quad 3$ rd edn. Oxford: Elsevier. Available at: https://books.google.com/books?id=BriOO0E667AC\&pgis=1.

Munkejord, S. T., Jakobsen, J. P., Austegard, A. and Mølnvik, M. J. (2010) 'Thermo- and fluiddynamical modelling of two-phase multi-component carbon dioxide mixtures', International Journal of Greenhouse Gas Control, 4(4), pp. 589-596. doi: 10.1016/j.ijggc.2010.02.003.

Munkejord, S. T. and Hammer, M. (2015) 'Depressurization of $\mathrm{CO}_{2}$-rich mixtures in pipes: 
Two-phase flow modelling and comparison with experiments', International Journal of Greenhouse Gas Control, 37, pp. 398-411. doi: 10.1016/j.ijggc.2015.03.029.

Munkejord, S. T., Hammer, M. and Løvseth, S. W. (2016) ' $\mathrm{CO}_{2}$ transport: Data and models A review', Applied Energy, 169, pp. 499-523. doi: 10.1016/j.apenergy.2016.01.100.

National Grid (2021) Gas transmission - Land and assets. Available at: https://www.nationalgrid.com/uk/gas-transmission/land-and-assets (Accessed: 21 March 2021).

National Transportation Safety Board (2003) Pipeline Accident Report: Natural Gas Pipeline Rupture and Fire Near Carlsbad, New Mexico, August 19, 2000. Available at: https://www.ntsb.gov/investigations/AccidentReports/Reports/PAR0301.pdf.

Oke, A., Mahgerefteh, H., Economou, I. and Rykov, Y. (2003) 'A transient outflow model for pipeline puncture', Chemical Engineering Science, 58(20), pp. 4591-4604. doi: 10.1016/S0009-2509(03)00338-5.

Oke, A. (2004) An Efficient Numerical Simulation for Modelling Outflow Following Rupture or Puncture of Pressurised Pipeline Networks. PhD thesis. University College London. Available at: https://search.proquest.com/docview/1752519901/?pq-origsite=primo.

Oland, C. B., Rose, S. D., Grant, H. L., Lower, M. D., Spann, M. A., Kirkpatrick, J. R. and Sulfredge, C. D. (2012) Studies for the requirements of automatic and remotely controlled shutoff valves on hazardous liquids and natural gas pipelines with respect to public and environmental safety, Oak Ridge National Laboratory. Available at: https://www.osti.gov/servlets/purl/1373899 (Accessed: 23 February 2021).

Ord, S. C. (2006) 'Water hammer - Do we need to protect against it? How to predict it and prevent it damaging pipelines and equipment', Institution of Chemical Engineers Symposium Series, (151), pp. 230-249.

Paillère, H., Corre, C. and García Cascales, J. R. (2003) 'On the extension of the AUSM+ scheme to compressible two-fluid models', Computers and Fluids, 32(6), pp. 891-916. doi: 10.1016/S0045-7930(02)00021-X.

Patchigolla, K. and Oakey, J. E. (2013) 'Design Overview of High Pressure Dense Phase $\mathrm{CO}_{2}$ 
Pipeline Transport in Flow Mode', Energy Procedia, 37, pp. 3123-3130. doi: 10.1016/j.egypro.2013.06.198.

Peng, D. Y. and Robinson, D. B. (1976) 'A new two-constant equation of state', Industrial and Engineering Chemistry Fundamentals, 15(1), pp. 59-64. doi: 10.1021/i160057a011.

Pham, L. H. H. P. and Rusli, R. (2016) 'A review of experimental and modelling methods for accidental release behaviour of high-pressurised $\mathrm{CO}_{2}$ pipelines at atmospheric environment', Process Safety and Environmental Protection, 104, pp. 48-84. doi: 10.1016/j.psep.2016.08.013.

Picard, D. J. and Bishnoi, P. R. (1987) 'Calculation of the thermodynamic sound velocity in two-phase multicomponent fluids', International Journal of Multiphase Flow, 13(3), pp. 295308. doi: 10.1016/0301-9322(87)90050-4.

Picard, D. J. and Bishnoi, P. R. (1988) 'The importance of real-fluid behavior and nonisentropic effects in modeling decompression characteristics of pipeline fluids for application in ductile fracture propagation analysis', The Canadian Journal of Chemical Engineering, 66(1), pp. 312. doi: $10.1002 /$ cjce. 5450660101 .

Pipeline and Hazardous Materials Safety Administration (2017) Pacific Gas \& Electric pipeline rupture in San Bruno, CA, U.S. Department of Transportation's Pipeline and Hazardous Materials Safety Administration. Available at: https://www.phmsa.dot.gov/safetyawareness/pipeline/pacific-gas-electric-pipeline-rupture-san-bruno-ca (Accessed: 3 May 2021).

Pipeline and Hazardous Materials Safety Administration (2021) Pipeline Incident 20 Year Trends, U.S. Department of Transportation's Pipeline and Hazardous Materials Safety Administration. Available at: https://www.phmsa.dot.gov/data-andstatistics/pipeline/pipeline-incident-20-year-trends (Accessed: 21 March 2021).

Ramírez-Camacho, J. G., Carbone, F., Pastor, E., Bubbico, R. and Casal, J. (2017) 'Assessing the consequences of pipeline accidents to support land-use planning', Safety Science, 97, pp. 34-42. doi: 10.1016/j.ssci.2016.01.021.

Ramsay, J. O., ten Berge, J. and Styan, G. P. H. (1984) 'Matrix correlation', Psychometrika, 
49(3), pp. 403-423. doi: 10.1007/BF02306029.

Richardson, S. and Saville, G. (1994) 'Isle of Grain pipeline depressurisation tests', Offshore Technology Report- .... Available at: http://www.opengrey.eu/item/display/10068/670459.

Roe, P. L. (1981) 'Approximate Riemann solvers, parameter vectors, and difference schemes', Journal of Computational Physics, 43(2), pp. 357-372. doi: 10.1016/0021-9991(81)90128-5.

Roe, P. L. (1985) 'Some contributions to the modelling of discontinuous flows', Lectures Notes in Applied Mathematics, 22, pp. 163-193. Available at: http://adsabs.harvard.edu/abs/1985ams..conf..163R.

Rohsenow, W., Hartnett, J. and Cho, Y. (1998) Handbook of heat transfer (Vol. 3).

Roy, B. and Bouyssou, D. (1986) 'Comparison of two decision-aid models applied to a nuclear power plant siting example', European Journal of Operational Research, 25(2), pp. 200-215. doi: 10.1016/0377-2217(86)90086-X.

Ryder, A. (1997) 'Environmental Impact Assessment in the Pipeline Industry'. Available at: https://inis.iaea.org/collection/NCLCollectionStore/_Public/29/045/29045753.pdf?r=1\&r=1.

Sabio, N., Kostin, A., Guillén-Gosálbez, G. and Jiménez, L. (2012) 'Holistic minimization of the life cycle environmental impact of hydrogen infrastructures using multi-objective optimization and principal component analysis', International Journal of Hydrogen Energy, 37(6), pp. 5385-5405. doi: 10.1016/j.ijhydene.2011.09.039.

Saha, P. (1997) Modelling the dynamic response of emergency shut-down valves following full bore rupture of long pipelines. $\mathrm{PhD}$ thesis. University College London.

Saville, G., Richardson, S. M. and Barker, P. (2004) 'Leakage in ethylene pipelines', Process Safety and Environmental Protection, 82(1), pp. 61-68. doi: 10.1205/095758204322777679.

Semin and Bakar, R. A. (2008) 'A Technical Review of Compressed Natural Gas as an Alternative Fuel for Internal Combustion Engines', American Journal of Engineering and Applied Sciences, 1(4), pp. 302-311. doi: 10.3844/ajeassp.2008.302.311.

Shah, M. M. (1979) 'A general correlation for heat transfer during film condensation inside pipes', International Journal of Heat and Mass Transfer, 22(4), pp. 547-556. doi: 
10.1016/0017-9310(79)90058-9.

Smith, P. and Zappe, R. W. (2004) Valve selection handbook, Valve Selection Handbook: Engineering Fundamentals for Selecting the Right Valve Design for Every Industrial Flow Application: Fifth Edition. Edited by P. Smith. Elsevier. doi: 10.1016/B978-0-7506-77172.X5000-7.

Soave, G. (1972) 'Equilibrium constants from a modified Redlich-Kwong equation of state', Chemical Engineering Science, 27(6), pp. 1197-1203. doi: 10.1016/0009-2509(72)80096-4.

State Council of the People's Republic of China (2016) The 13th Five Year Plan of China. Available at: http://www.gov.cn/zhengce/content/2016-12/27/content_5153411.htm (Accessed: 3 March 2021).

Swaffied, J. A. and Boldy, A. P. (1993) Pressure surge and duct systems. Avebury Technical.

Teng, L., Li, Y., Zhao, Q., Wang, W., Hu, Q., Ye, X. and Zhang, D. (2016) 'Decompression characteristics of $\mathrm{CO}_{2}$ pipelines following rupture', Journal of Natural Gas Science and Engineering, 36, pp. 213-223. doi: 10.1016/j.jngse.2016.10.026.

Terenzi, A. (2005) 'Influence of real-fluid properties in modeling decompression wave interacting with ductile fracture propagation', Oil and Gas Science and Technology, 60(4), pp. 711-719. doi: 10.2516/ogst:2005050.

Thorley, A. R. D. (1989) 'Check Valve Behavior Under Transient Flow Conditions: A Stateof-the-Art Review', Journal of Fluids Engineering, 111(2), pp. 178-183. doi: $10.1115 / 1.3243620$.

Titarev, V. A. and Toro, E. F. (2005) 'MUSTA schemes for multi-dimensional hyperbolic systems: Analysis and improvements', International Journal for Numerical Methods in Fluids, 49(2), pp. 117-147. doi: 10.1002/fld.980.

Toro, E. F. (2003) 'Multi-stage predictor-corrector fluxes for hyperbolic equations', Isaac Newton Institute for Mathematical Sciences Preprint Series.

Toro, E. F. (2009) Riemann solvers and numerical methods for fluid dynamics: A practical introduction, Riemann Solvers and Numerical Methods for Fluid Dynamics: A Practical 
Introduction. doi: 10.1007/b79761.

Transportation Research Board (2004) Transmission Pipelines and Land Use: A Risk-Informed Approach -- Special Report 281. Washington, DC: The National Academies Press. doi: $10.17226 / 11046$.

Tušar, T. (2014) Visualizing Solution Sets in Multiobjective Optimization. PhD thesis. Jožef Stefan International Postgraduate School.

Versteeg, H. K., Malalasekera, W., Orsi, G., Ferziger, J. H., Date, A. W. and Anderson, J. D. (1995) An Introduction to Computational Fluid Dynamics - The Finite Volume Method, Fluid flow handbook. McGraw-Hill $\quad \ldots . \quad$ Available at: http://scholar.google.com/scholar?hl=en\&btnG=Search\&q=intitle:Computational+fluid+dyna mics.+The+basics+with+applications\#6\%5Cnhttp://scholar.google.com/scholar?hl=en\&btnG $=$ Search \&q=intitle:Computational+Fluid+Dynamics: + The+Basics+with+Applications +1995 $\% 23$.

Walas, S. M. (1985) Phase Equilibria in Chemical Engineering., Phase Equilib in Chem Eng. doi: 10.1016/c2013-0-04304-6.

Wang, H., Olhofer, M. and Jin, Y. (2017) 'A mini-review on preference modeling and articulation in multi-objective optimization: current status and challenges', Complex \& Intelligent Systems, 3(4), pp. 233-245. doi: 10.1007/s40747-017-0053-9.

Wright, S. J. (2016) Optimization, Encyclopedia Britannica. Available at: https://www.britannica.com/science/optimization (Accessed: 11 April 2021).

Wu, S. (1998) Steady-state simulation and fuel cost minimization of gas pipeline networks. $\mathrm{PhD}$ thesis. University of Houston.

Wylie, E. B. and Streeter, V. L. (1978) Fluid transients. New York: McGraw-Hill International Book Co. doi: 10.1115/1.3448743.

Wylie, E. B. and Streeter, V. L. (1993) 'Fluid Transients in Systems', PrenticeHall Upper Saddle River NJ, pp. 287-362. doi: 10.1061/(ASCE)HY.1943-7900.0001067.

Wylie, E. B., Streeter, V. L. and Suo, L. (1993) Fluid transients in systems. Englewood Cliffs, 


\section{DEPARTMENT OF CHEMICAL ENGINEERING}

NJ: Prentice Hall. Available at: http://www-personal.umich.edu/ ebw/Appendix D Programs/README.DOC.

Xu, B. P., Jie, H. E. and Wen, J. X. (2014) 'A pipeline depressurization model for fast decompression and slow blowdown', International Journal of Pressure Vessels and Piping, 123, pp. 60-69. doi: 10.1016/j.ijpvp.2014.07.003.

Yang, J.-B. (2000) 'Minimax reference point approach and its application for multiobjective optimisation', European Journal of Operational Research, 126(3), pp. 541-556. doi: 10.1016/S0377-2217(99)00309-4.

Yang, J. B. (1996) Multiple criteria decision making methods and applications. Changsha: Hunan Publishing House.

Yang, X.-S. (2010) 'Engineering Optimization', in Engineering Optimization. Hoboken, NJ, USA: John Wiley \& Sons, Inc., pp. 15-28. doi: 10.1002/9780470640425.ch2.

Yeom, G. S. and Chang, K. S. (2013) 'A modified HLLC-type Riemann solver for the compressible six-equation two-fluid model', Computers and Fluids, 76, pp. 86-104. doi: 10.1016/j.compfluid.2013.01.021.

Zheng, W. (2018) Numerical Modelling of the Rapid Depressurisation and Outflow of High Pressure Containments in the Framework of Carbon Capture and Sequestration. PhD thesis. University College London. Available at: http://discovery.ucl.ac.uk/10049915/.

Zio, E. and Bazzo, R. (2011) 'Level Diagrams analysis of Pareto Front for multiobjective system redundancy allocation', Reliability Engineering and System Safety, 96(5), pp. 569-580. doi: 10.1016/j.ress.2010.12.016.

Zucrow, M. J. and Hoffman, J. D. (1976) Gas Dynamics. doi: 10.1201/9781315274065-11. 